\title{
Light Water Reactor Sustainability Program
}

\section{Nuclear Power Plant Component Flooding Fragility Research}

C. L. Pope, B. Savage, S. Jash, B. Johnson, C. Muchmore, L. Nichols, E. Ryan, S. Suresh, A. Tahhan, R. Tuladhar, A. Wells, C. Smith

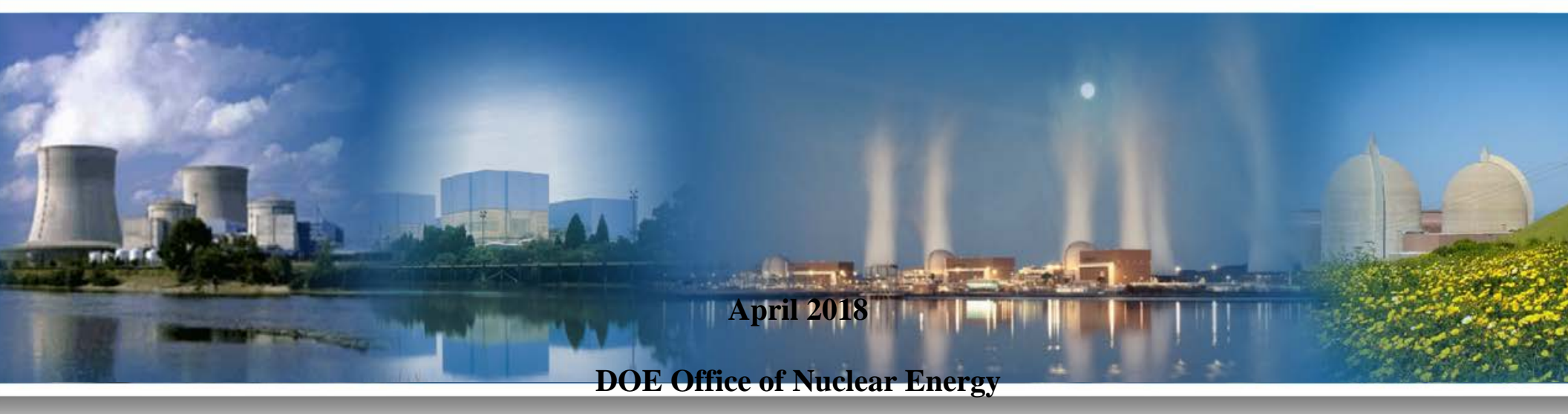





\section{DISCLAIMER}

This information was prepared as an account of work sponsored by an agency of the U.S. Government. Neither the U.S. Government nor any agency thereof, nor any of their employees, makes any warranty, expressed or implied, or assumes any legal liability or responsibility for the accuracy, completeness, or usefulness, of any information, apparatus, product, or process disclosed, or represents that its use would not infringe privately owned rights. References herein to any specific commercial product, process, or service by trade name, trade mark, manufacturer, or otherwise, does not necessarily constitute or imply its endorsement, recommendation, or favoring by the U.S. Government or any agency thereof. The views and opinions of authors expressed herein do not necessarily state or reflect those of the U.S. Government or any agency thereof. 


\section{Light Water Reactor Sustainability Program}

\section{Nuclear Power Plant Component Flooding \\ Fragility Research}

C. L. Pope - Idaho State University

B. Savage - Idaho State University

S. Jash - Idaho State University

B. Johnson - Idaho State University

C. Muchmore - Idaho State University

L. Nichols - Idaho State University

E. Ryan - Idaho State University

S. Suresh - Idaho State University

A. Tahhan - Idaho State University

R. Tuladhar - Idaho State University

A. Wells - Idaho State University

C. L. Smith - INL

April 2018

Idaho National Laboratory

Idaho Falls, Idaho 83415

http://www.inl.gov/lwrs

Prepared for the

U.S. Department of Energy

Office of Nuclear Energy

Under DOE Idaho Operations Office

Contract DE-AC07-05ID14517 



\begin{abstract}
This report describes progress on Nuclear Power Plant component flooding fragility research conducted at the Component Flooding Evaluation Laboratory (CFEL). The progress includes additional steering committee membership and invitations, performance improvements to the Portal Evaluation Tank (PET), PET based experiments using industrial steel doors, pipe rupture spray pattern characterization, coupling the smoothed particle hydrodynamics (SPH) code Neutrino and the Idaho National Laboratory (INL) developed Risk Analysis Virtual Environment (RAVEN), continued development of component fragility mathematical models, and wave impact simulation device development, testing, and analysis.
\end{abstract}




\section{CONTENTS}

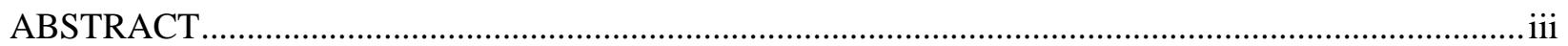

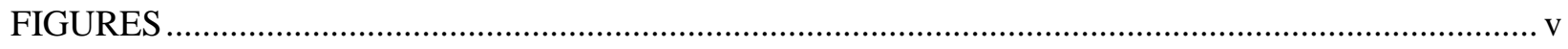

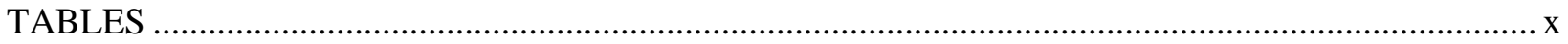

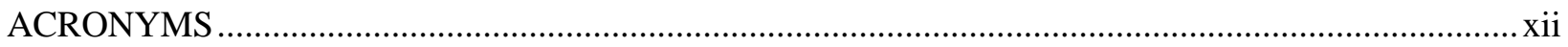

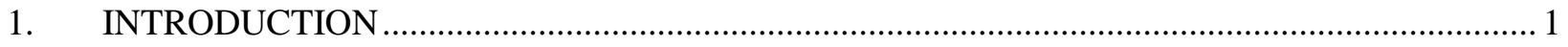

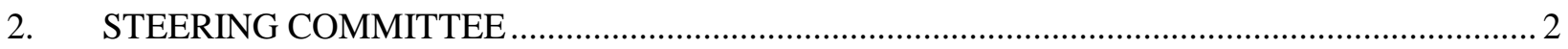

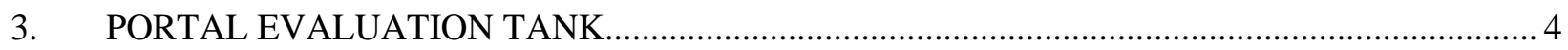

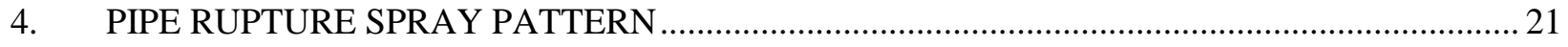

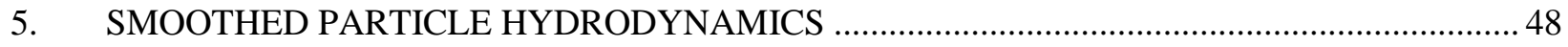

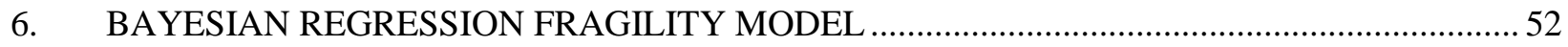

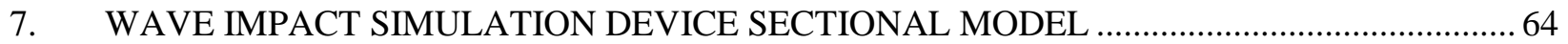

8. WAVE IMPACT SIMULATION DEVICE STRUCTURAL ANALYSIS .....................................95

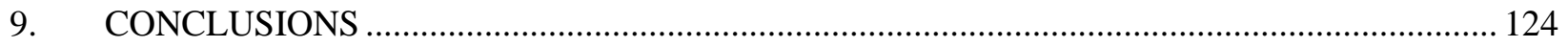

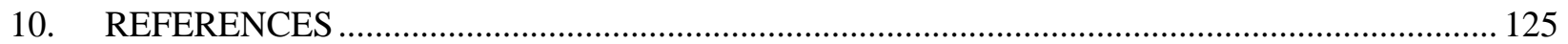




\section{FIGURES}

Figure 1. Industrial steels doors involved in the Fukushima flooding.................................................. 1

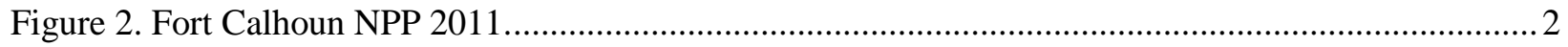

Figure 3. Component Flooding Evaluation Steering Committee Charter................................................... 3

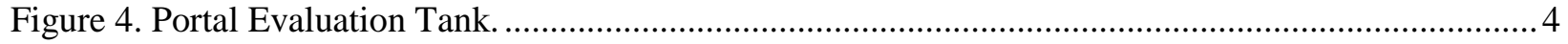

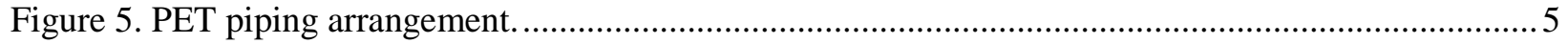

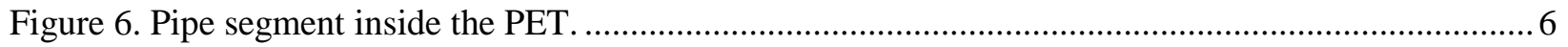

Figure 7. PET piping and instrumentation diagram............................................................................ 7

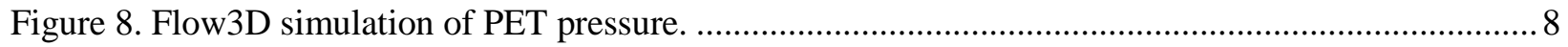

Figure 9. Flow3D PET pressure with return open................................................................................ 9

Figure 10. Hollow-core door experiments - PET water depth................................................................ 10

Figure 11. Hollow-Core door experiment \#6 - flow information. ............................................................. 10

Figure 12. Metal door experiments \#1, \#2 \& \#3 - PET water depth....................................................... 11

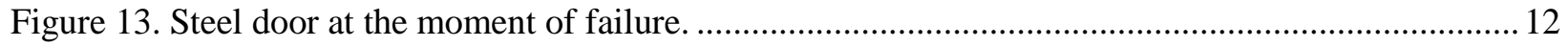

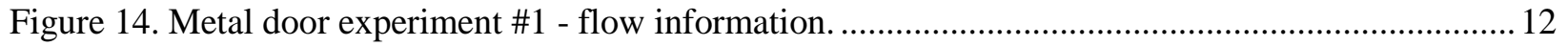

Figure 15. Metal door experiment \#2 - flow information. ......................................................................... 13

Figure 16. Metal door experiment \#3 - flow information. .......................................................................... 13

Figure 17. Metal door experiment \#4 \& \#5 - PET water depth. ................................................................. 14

Figure 18. Metal door experiment \#4 - flow information. ...................................................................... 15

Figure 19. Metal door experiment \#5 - flow information. ....................................................................... 15

Figure 20. Metal door experiment \#6, \#7 \& \#8 - PET water depth. ............................................................ 16

Figure 21. Metal door experiment \#9 - PET water depth. ....................................................................... 17

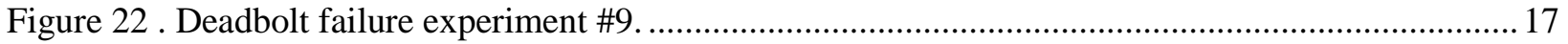

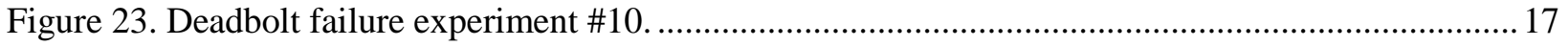

Figure 24. Metal door experiment \#11 - flow information ......................................................................... 18

Figure 25. Experiment \#11 - post-experiment door damage....................................................................... 19

Figure 26. Deadbolt comparison - experiments \#9 \#10 (left-typical), experiment \#11 (right).................. 19

Figure 27. Experiment \#12 - PET water height. ...................................................................................... 20

Figure 28. Metal door experiment \#12 - flow information. ...................................................................... 21

Figure 29. Needed emitter with spray pattern capability. .......................................................................2

Figure 30. Pump curve operating point calculations........................................................................ 25

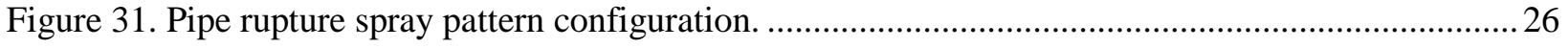

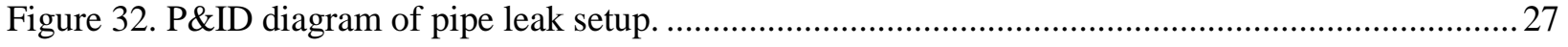

Figure 33. Longitudinally slotted galvanized nipples......................................................................... 28 


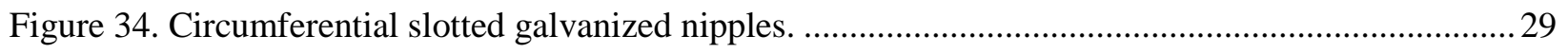

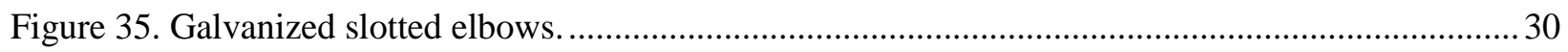

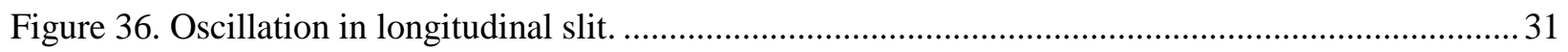

Figure 37. Larger view of longitudinal spray with no valves. ............................................................... 32

Figure 38. Fan spray from a circumferential crack, 3-inch pipe............................................................. 33

Figure 39. Elevation view of the circumferential spray........................................................................ 34

Figure 40. Fan spray from a circumferential crack, 2-inch pipe.......................................................... 35

Figure 41 Fan spray from a circumferential crack, 1-inch pipe............................................................ 36

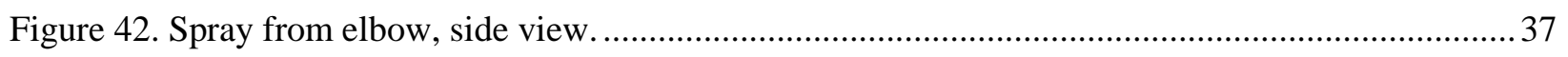

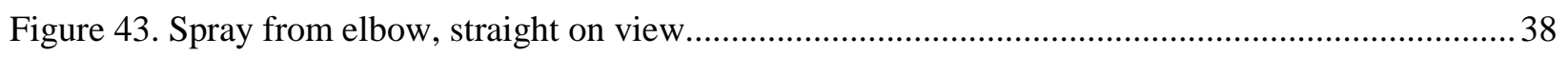

Figure 44. Type 2 leak from a longitudinal crack, close view........................................................... 40

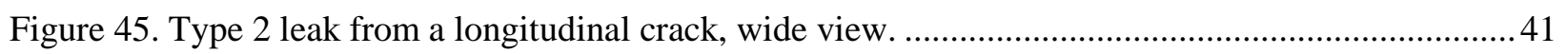

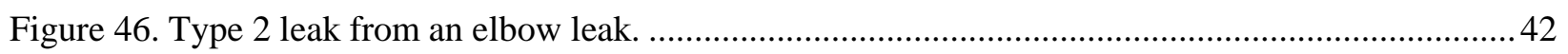

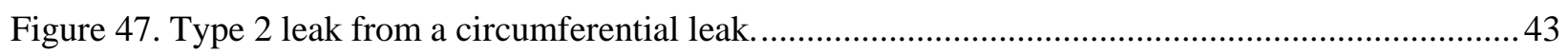

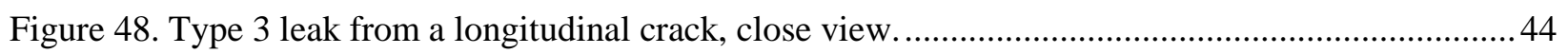

Figure 49. Type 3 leak from a longitudinal crack, wide view ............................................................. 45

Figure 50. Type 3 leak from a circumferential leak........................................................................... 46

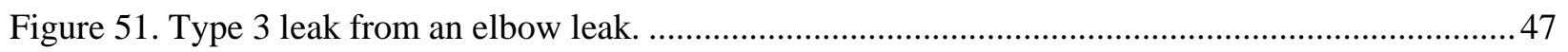

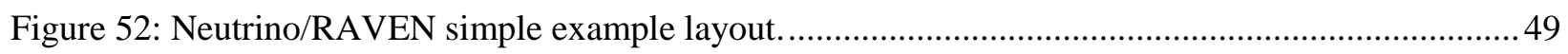

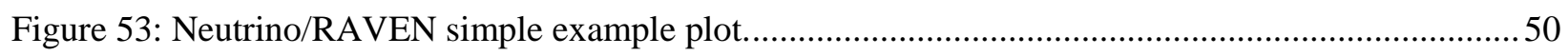

Figure 54. Horizontal orientation of benchmark experiment....................................................................5

Figure 55. Vertical orientation of benchmark experiment. ................................................................ 51

Figure 56. Neutrino SPH simulation for the horizontal orientation experiment......................................5 52

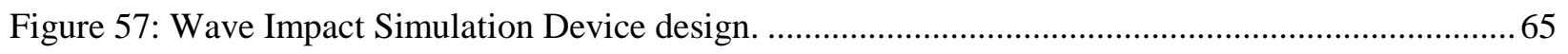

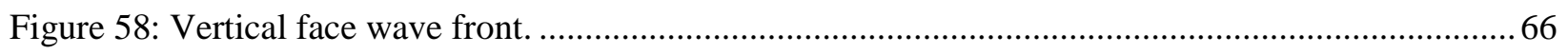

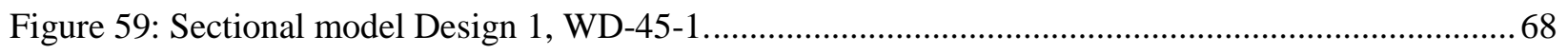

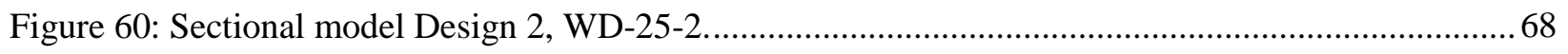

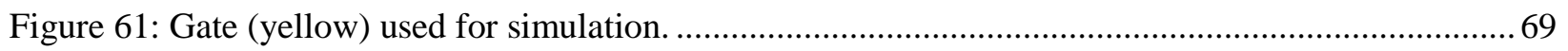

Figure 62: Representation of gate partially opening. .......................................................................... 70

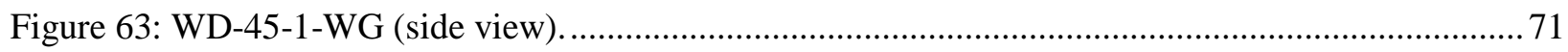

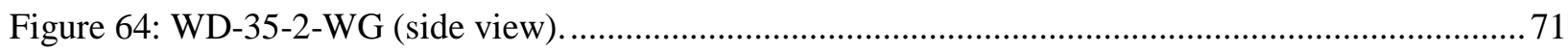

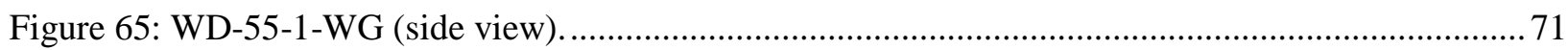

Figure 66: General layout of the sectional physical model..................................................................... 73

Figure 67: Layout of the physical experiment study............................................................................... 74

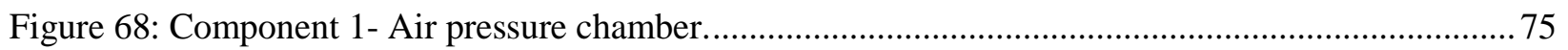


Figure 69: Diagram of pneumatic system for pipe model.................................................................... 75

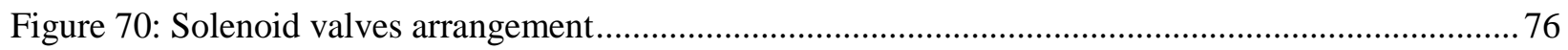

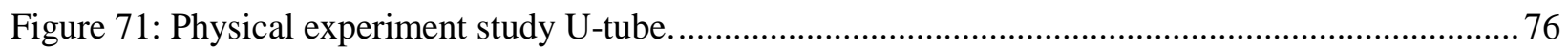

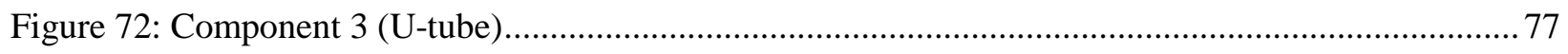

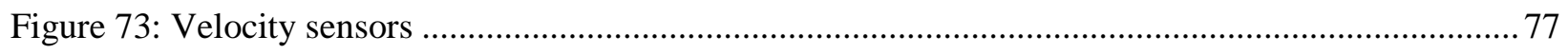

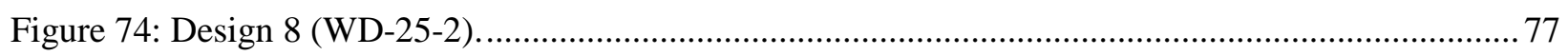

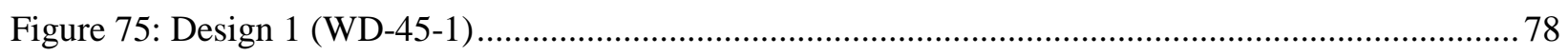

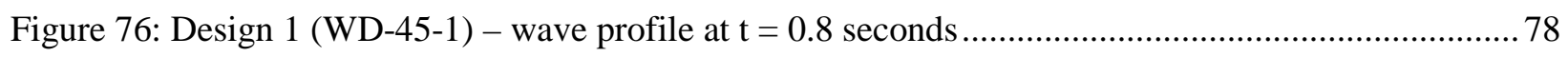

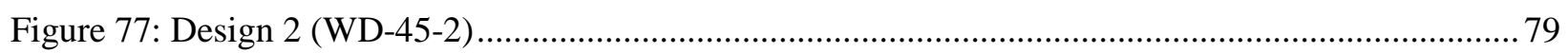

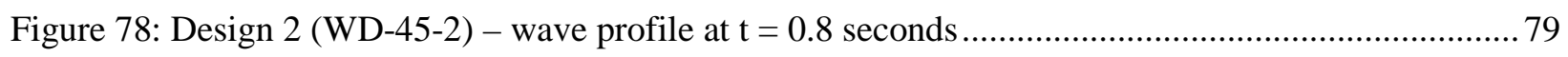

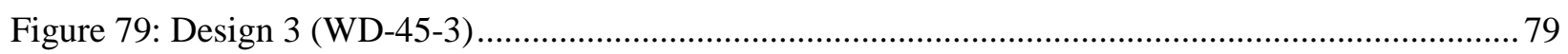

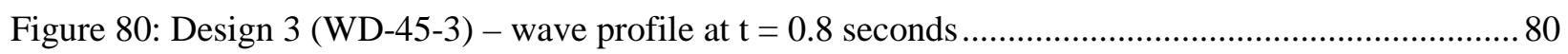

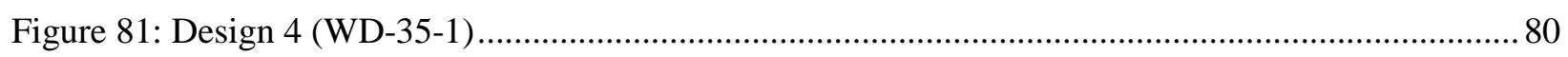

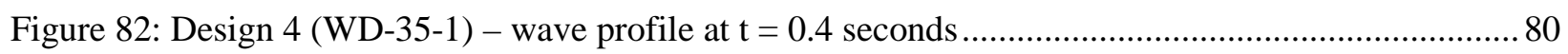

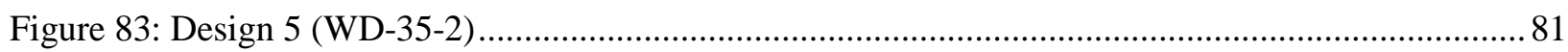

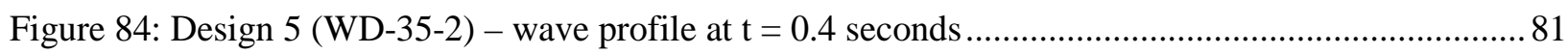

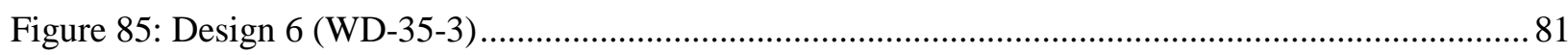

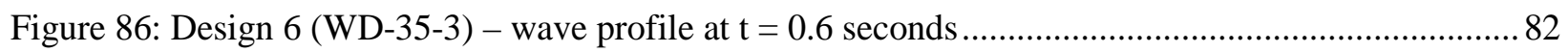

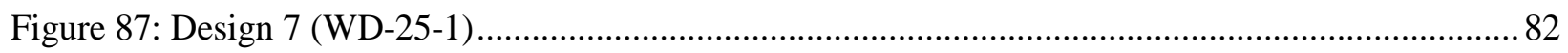

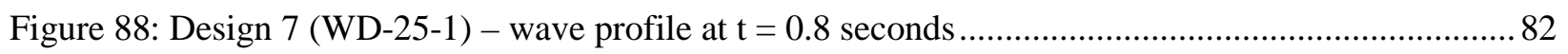

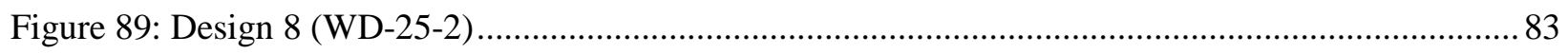

Figure 90: Design 8 (WD-25-2) - wave profile at $\mathrm{t}=0.85$ seconds ........................................................ 83

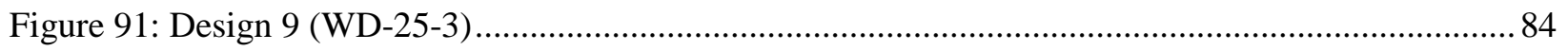

Figure 92: Design 9 (WD-25-3) - wave profile at $\mathrm{t}=0.9$ seconds ........................................................... 84

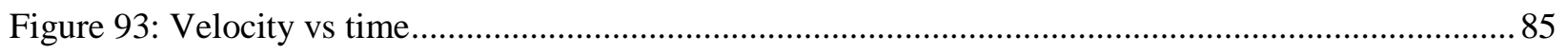

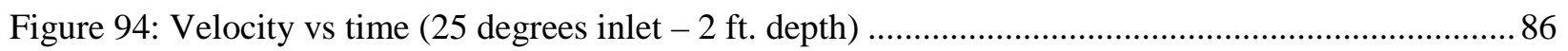

Figure 95: Velocity - vs - time (without gates) for geometries with: (a) 45 degrees; (b) 35

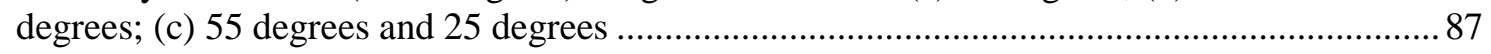

Figure 96: Wave profile generated by an inlet pressure of 2.75 psi ..................................................... 88

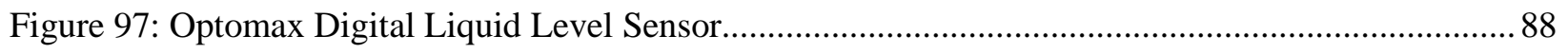

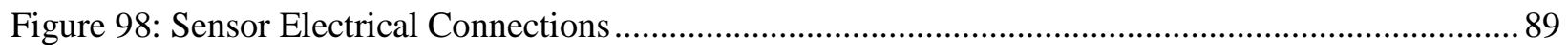

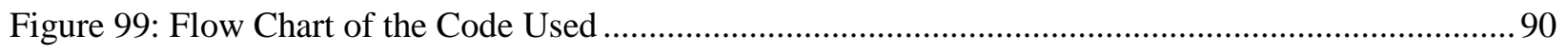

Figure 100: Arduino Mega 2560 Micro-controller ................................................................................... 91

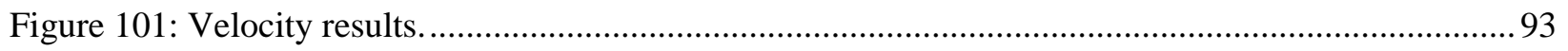

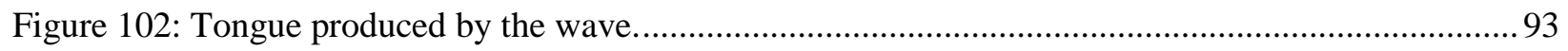




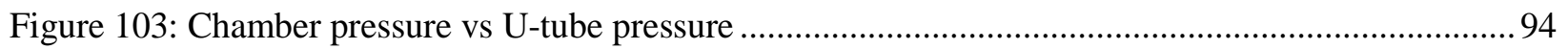

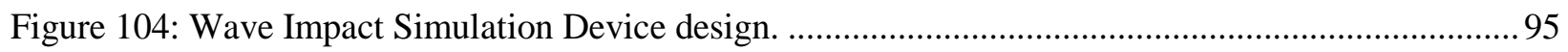

Figure 105. Overview of the 2-1/2' x 10' x 3/32” plate system. ............................................................ 96

Figure 106. Von-Mises stress of a 2-1/2' x 10' x 3/32" plate with long edges fixed under 0.433 psi.........96

Figure 107. Deflection of a 2-1/2' x 10' x 3/32" plate with long edges fixed under 0.433 psi....................97

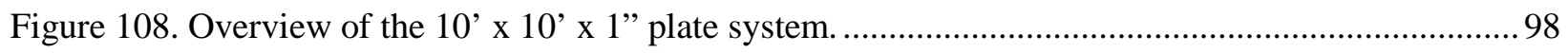

Figure 109. Von-Mises stress of a 10' x 10' x 1" plate with long edges fixed under 0.433 psi...................98

Figure 110. Deflection stress of a 10' x 10' x 1" plate with long edges fixed under 0.433 psi.................... 99

Figure 111. Von-Mises stress of a 10' x 10' x 3/8" plate with three fixed edges under 0.433 psi..............100

Figure 112. Deflection of a 10' x 10' x 3/8" plate with three fixed edges under 0.433 psi. ...................... 100

Figure 113. Von-Mises stress of a 10' x 10' x 3/8" plate with three fixed edges under 0.433 psi..............101

Figure 114. Deflection of a 10' x 10' x 1" plate with three fixed edges under 0.433 psi........................ 101

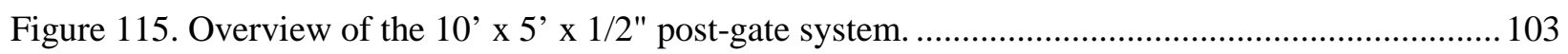

Figure 116. Von-Mises stress for nine 10' x 5' x 1/2" plates under a 1.3 psi static load...........................103

Figure 117. Deflection for the nine 10' x 5' x 1/2" plates under a 1.3 psi static load................................ 104

Figure 118. Von-Mises stress for the nine 10' x 5' x 1/2" plates under static and dynamic loading.......... 104

Figure 119. Onset of deflection of the 10' x 5' x 1/2" plates under static and dynamic loading................ 105

Figure 120. Cessation of deflection of the $10^{\prime}$ x 5' x 1/2" plates under static and dynamic loading.......... 106

Figure 121. Bottom view of crossbeams attached to a 10' x 5' x 3/8” plate. ..........................................107

Figure 122. Von-Mises stress of a 10' x 5' x 3/8" plate with crossbeams under 1.3 psi. ........................ 107

Figure 123. Deflection of a 10' x 5' x 3/8" plate with crossbeams under 1.3 psi..................................... 108

Figure 124. A 10’ x 5' x 3/8” plate supported using 1/4” thick, notched steel........................................ 108

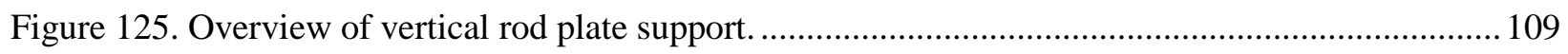

Figure 126. Close-up of vertical rod and washer attachments............................................................... 109

Figure 127. Von-Mises stress of a 10' x 5' x 3/8" plate under 1.3 psi with two support rods.................... 110

Figure 128. Deflection of a 10' x 5' x 3/8" plate under 1.3 psi with two support rods............................. 110

Figure 129. Close-up of expanded rivet nut after installation............................................................... 111

Figure 130. Overview of threaded rivet nut and rod plate supporting system........................................ 111

Figure 131. Von-Mises stress of a 10' x 5' x 3/8" plate under 1.3 psi with rivet nut/rod supports. ...........112

Figure 132. Deflection of a 10' x 5' x 3/8" plate under 1.3 psi with rivet nut/rod supports......................112

Figure 133. Isometric view of gate plate attached to keyed shaft............................................................. 113

Figure 134. Deflection of a 1" keyed shaft with a 1/4" rectangular gate under 0.433 psi......................115

Figure 135. Deflection of a 1-1/4" keyed shaft with a 1/4" rectangular gate under 0.433 psi ................. 116

Figure 136. Deflection of a 1-1/2” keyed shaft with a 3/8" rectangular gate under 0.433 psi .................117

Figure 137. Deflection of a 1-3/4” keyed shaft with a 3/8" rectangular gate under 0.433 psi................. 118 
Figure 138. Von-Mises stress of a 1-3/4” keyed shaft with a 3/8" rectangular gate under 0.433 psi ........118

Figure 139. Isometric view of custom hinges supporting closed gate. ................................................. 119

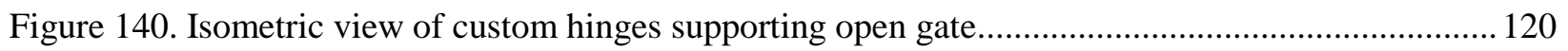

Figure 141. Close-up of custom machined hinge (left) and installation (right)..................................... 120

Figure 142. Deflection of 1-3/4" keyed shaft, 3/8" rectangular gate, and custom hinges under

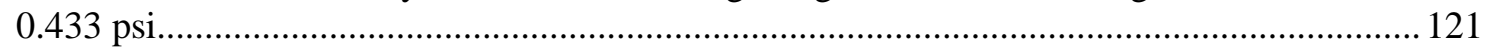

Figure 143. Von-Mises stress of 1-3/4” keyed shaft, 3/8" thick gate, and custom hinges under

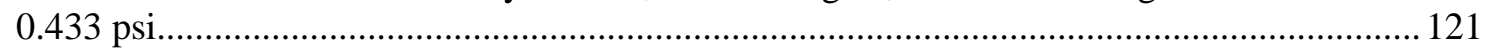

Figure 144. Simulation of chosen prototype components assembled within the WISD housing. ............ 122

Figure 145. Simulation of the energized electromagnet holding gate in closed position. ........................ 123

Figure 146. Simulation of the de-energized electromagnet releasing gate to opening position. ...............124 


\section{TABLES}

Table 1. Number of Piping Failures for Various Pipe Sizes and Pipe Size Categories ............................ 22

Table 2. Number of Piping Failures by Type of Failure ....................................................................... 22

Table 3. Number by Piping Failures for Each Failure Mechanism Category ............................................ 23

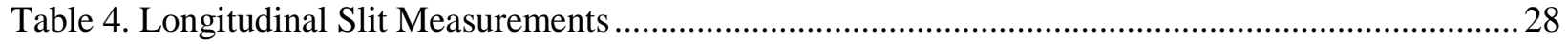

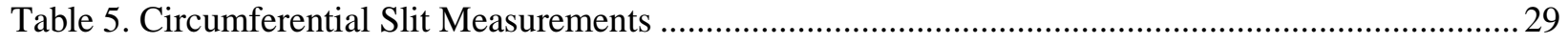

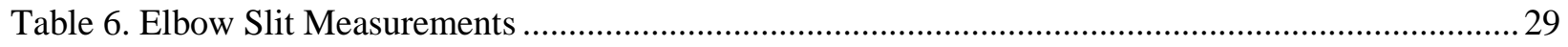

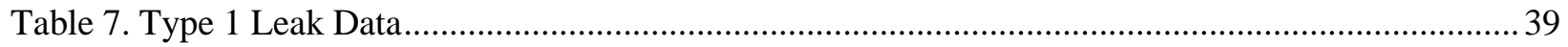

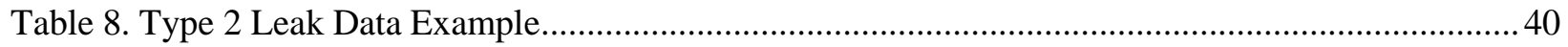

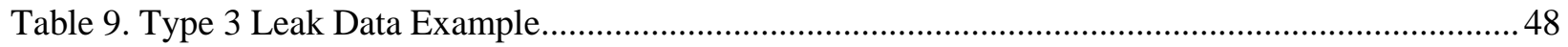

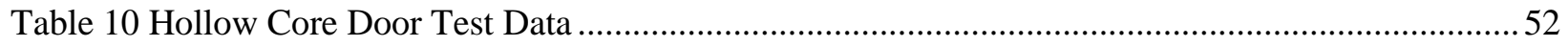

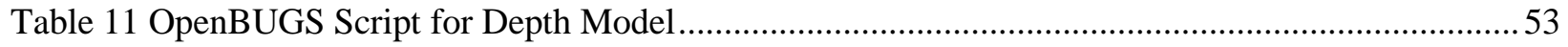

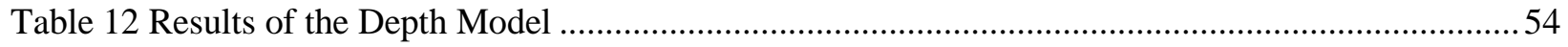

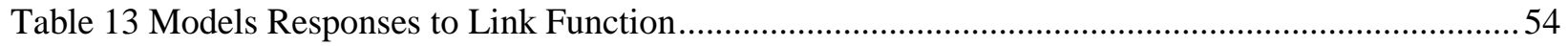

Table 14 Errors Observed While Using Different Link Functions ........................................................... 55

Table 15 Information on DIC and Other Parameters of the Seven Models ............................................ 56

Table 16 Data on Inward Swinging Failures for Hollow Core Doors ...................................................... 56

Table 17 OpenBUGS Script for Exponential Aleatory Model for Door Failure with Jeffreys Prior.......... 57

Table 18 Results for Standard Exponential Script with Jeffreys Prior ……............................................... 57

Table 19 Result of Standard Exponential Script with Diffuse Normal Prior.............................................. 58

Table 20 OpenBUGS Script for Exponential Aleatory Model with Bayesian p-value Calculation ............59

Table 21 Summary of Coefficient Estimates, p-value, and DIC for Exponential Model for Hollow

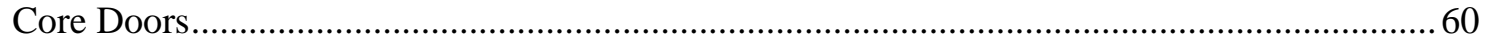

Table 22 OpenBUGS Script for Lognormal Model.............................................................................61

Table 23 Summary of Coefficient Estimates, p-value, and DIC for Lognormal Model for Hollow

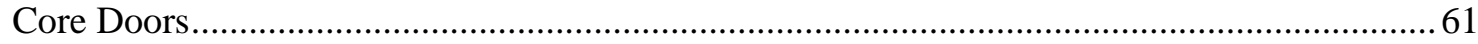

Table 24 Summary of Coefficient Estimates, p-value, and DIC for Normal Model for Hollow Core Doors.

Table 25 OpenBUGS Script of a Weibull Distribution with Influence on the Beta Parameter.....

Table 26 Summary of Coefficient Estimates, p-value, and DIC for Weibull Model with Environment Impact on Beta parameter for Hollow Core Door.

Table 27 Summary of Coefficient Estimates, p-value, and DIC for Weibull Model with Environment Impact on Lambda Parameter for Hollow Core Door. 64

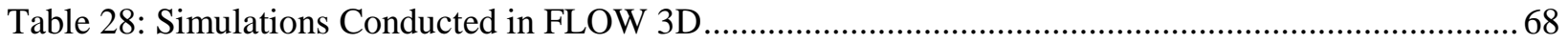

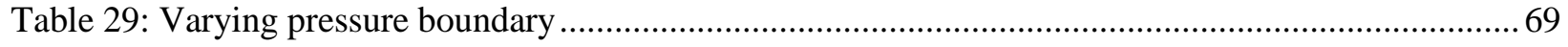

Table 30: Sectional Model Simulations Conducted Without Gates.......................................................... 70 


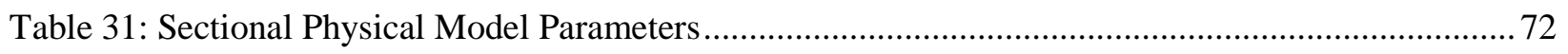

Table 32: Components of the Physical Sectional Model ........................................................................ 72

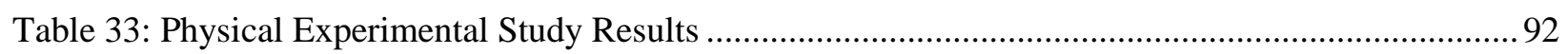

Table 34: Physical vs Theoretical Results for a Chamber Pressure of 2.75 psig (run 5) .......................... 94

Table 35. Standard Keyed Shaft Sizes (Grainger 2018) ....................................................................... 114 


\section{ACRONYMS}

$\begin{array}{ll}\text { BIC } & \text { Bayesian Information Criteria } \\ \text { CFD } & \text { Computational Fluid Dynamics } \\ \text { CFEL } & \text { Component Flooding Evaluation Laboratory } \\ \text { DIC } & \text { Deviance Information Criteria } \\ \text { E/C } & \text { Erosion/Corrosion } \\ \text { FV } & \text { Fatigue-Vibration } \\ \text { LBB } & \text { Leak Before Break } \\ \text { MCMC } & \text { Markov Chain Monte Carlo } \\ \text { NPP } & \text { Nuclear Power Plant } \\ \text { PET } & \text { Portal Evaluation Tank } \\ \text { RAVEN } & \text { Risk Analysis Virtual Environment } \\ \text { SKI } & \text { Swedish Nuclear Power Inspectorate } \\ \text { SPH } & \text { Smoothed Particle Hydrodynamics } \\ \text { WISD } & \text { Wave Impact Simulation Device }\end{array}$




\section{Nuclear Power Plant Component Flooding Fragility Research}

\section{INTRODUCTION}

The tsunami that struck the Fukushima Nuclear Plant complex in March 2011 served as a stark reminder of Nuclear Power Plant flooding hazards. The performance of various components, under flooding conditions is not well characterized which interferes with thorough risk analysis. For example, Figure 1 shows industrial steel doors involved in the Fukushima flooding event.

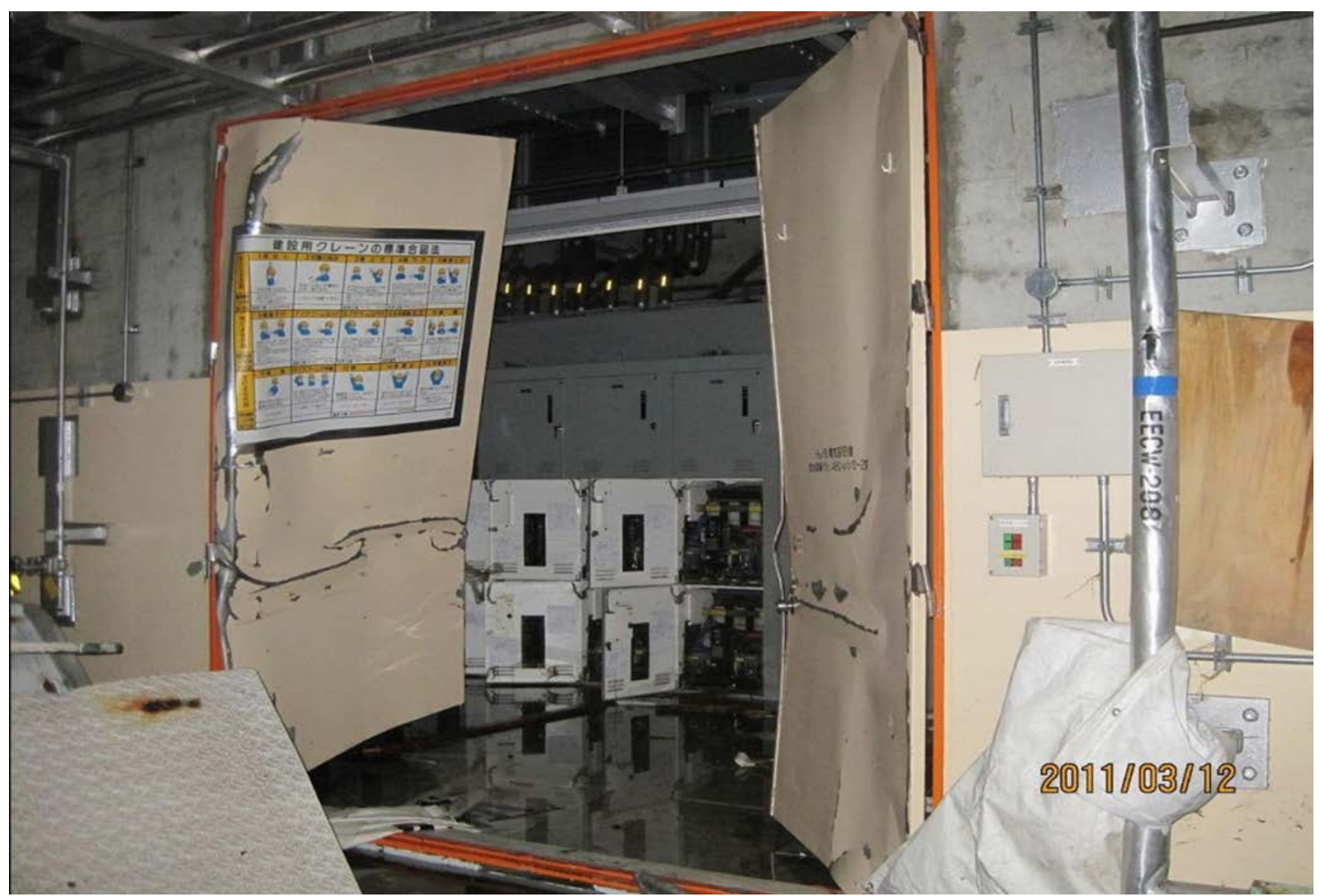

Figure 1. Industrial steels doors involved in the Fukushima flooding.

The lack of data and characterization regarding component performance under flooding conditions is additionally concerning since NPP flooding events are more common than one may expect. In the U.S., NPPs have faced various internal and external flooding events with the most notable being the 2011 Ft. Calhoun NPP flooding event shown in Figure 2. 


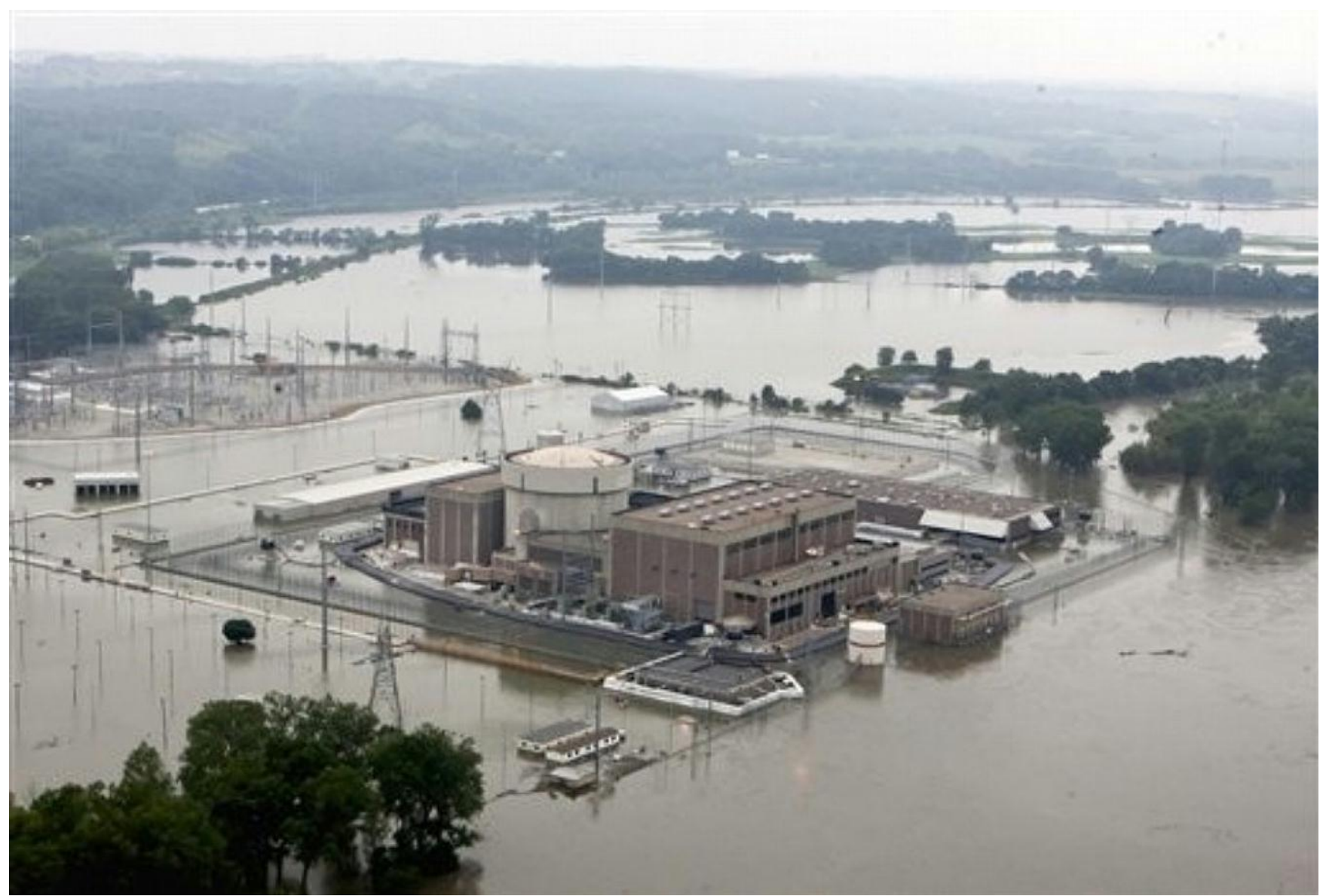

Figure 2. Fort Calhoun NPP 2011.

To help address the shortfall regarding component performance characterization, research is underway at the Component Flooding Evaluation Laboratory (CFEL). Research activities at CFEL include component-based flooding fragility experiments and analysis, mathematical model development, and flooding simulation (both numerical and physical) development. In the sections of this report that follow, updates are provided on progress related to CFEL research activities.

\section{STEERING COMMITTEE}

To help guide the selection of components for testing as well as other aspects of the component fragility testing protocol, a steering committee charter has been developed. The steering committee charter is shown in Figure 3. Initial steering committee member invitations were issued in the Fall of 2017. Additional committee member invitations were issued in the Spring of 2018. Members accepting the invitation as well as those invitations pending are shown in Figure 3. 


\section{Idaho State

\author{
U N I V E R S I T Y
}

College of Science and Engineering

921 South 8th Avenue, Stop 8065 - Pocatello, Idaho 83209-8065

\section{Component Flooding Evaluation Laboratory Steering Committee Charter} April 17, 2018

\section{Purpose}

The Component Flooding Evaluation Laboratory (CFEL) Steering Committee (CSC) provides technical review, strategic guidance, and oversight concurrence for component based flooding fragility experiment priorities and protocol, associated mathematical model application, and flooding simulation development strategies.

\section{Scope}

1. The CSC shall provide technical review of all proposed component flooding fragility experiments, fragility mathematical model approaches, and flooding simulation techniques.

2. The CSC technical review shall consider nuclear power industry impacts, nuclear power regulatory impacts, risk analysis strategy impacts, overall programmatic cost impacts, and proposed experiment safety implications.

3. The CSC shall provide strategic guidance for potential component flooding fragility experiments, fragility mathematical model and flooding simulation development strategies.

4. The CSC strategic guidance shall consider CSC member experience, nuclear power industry impacts, nuclear power regulatory impacts, risk analysis strategy impacts, and overall programmatic cost impacts.

5. The CSC shall periodically review results of component flooding fragility experiments.

Reporting, Membership, and Protocol

1. The CSC reports to the ISU Flooding Experiments Director (Dr. Chad Pope).

2. The CSC chair shall be a member of academia.

3. The CSC membership shall include at least one member representing nuclear power industry interests.

4. The CSC membership shall include at least one member representing risk assessment research interests.

5. The Department of Energy RISMC Pathway Lead shall be an ex-officio member of the CSC.

6. CSC membership shall be proposed by the CSC chair with concurrence by the RISMC Pathway Lead.

7. CSC meetings may be conducted in person or via electronic methods.

8. The CSC Chair shall confirm a quorum is present for each meeting where a quorum consists of at least two-thirds majority of committee members.

9. CSC concurrence requires at least two-thirds of the CSC membership and must include the CSC Chair.

10. In cases where unanimous CSC concurrence is not achieved, dissenting opinions must be provided to the RISMC Lead.

\author{
Members \\ Dr. Chad Pope, Idaho State University \\ Dr. Curtis Smith, Idaho National Laboratory \\ Mr. Alan Moldenhauer, Dominion Energy \\ Mr. Bentley Harwood, Idaho National Laboratory \\ Mr. Kelly Boodry, retired \\ John Weglian, Electric Power Research Institute \\ Charkas Hasan, Electric Power Research Institute \\ Thomas Aird, Nuclear Regulatory Commission
}

Chair
RISMC Pathway Lead
Member
Member
Invitation pending
Invitation pending
Invitation pending
Invitation Pending

Phone: (208) 282-3099 - Fax: (208) 282-4464 · wwow.isu.edu ISU is an Equal Opportunity Employer

Figure 3. Component Flooding Evaluation Steering Committee Charter. 


\section{PORTAL EVALUATION TANK}

The Portal Evaluation Tank (PET) is used to perform component flooding fragility experiments. The PET is a steel tank, with a semi-cylindrical shape; it has height of $8 \mathrm{ft}$. and a width of $8 \mathrm{ft}$.; the diameter of the circular section is $8 \mathrm{ft}$. The tank has a square opening section for the installation of components to be tested.

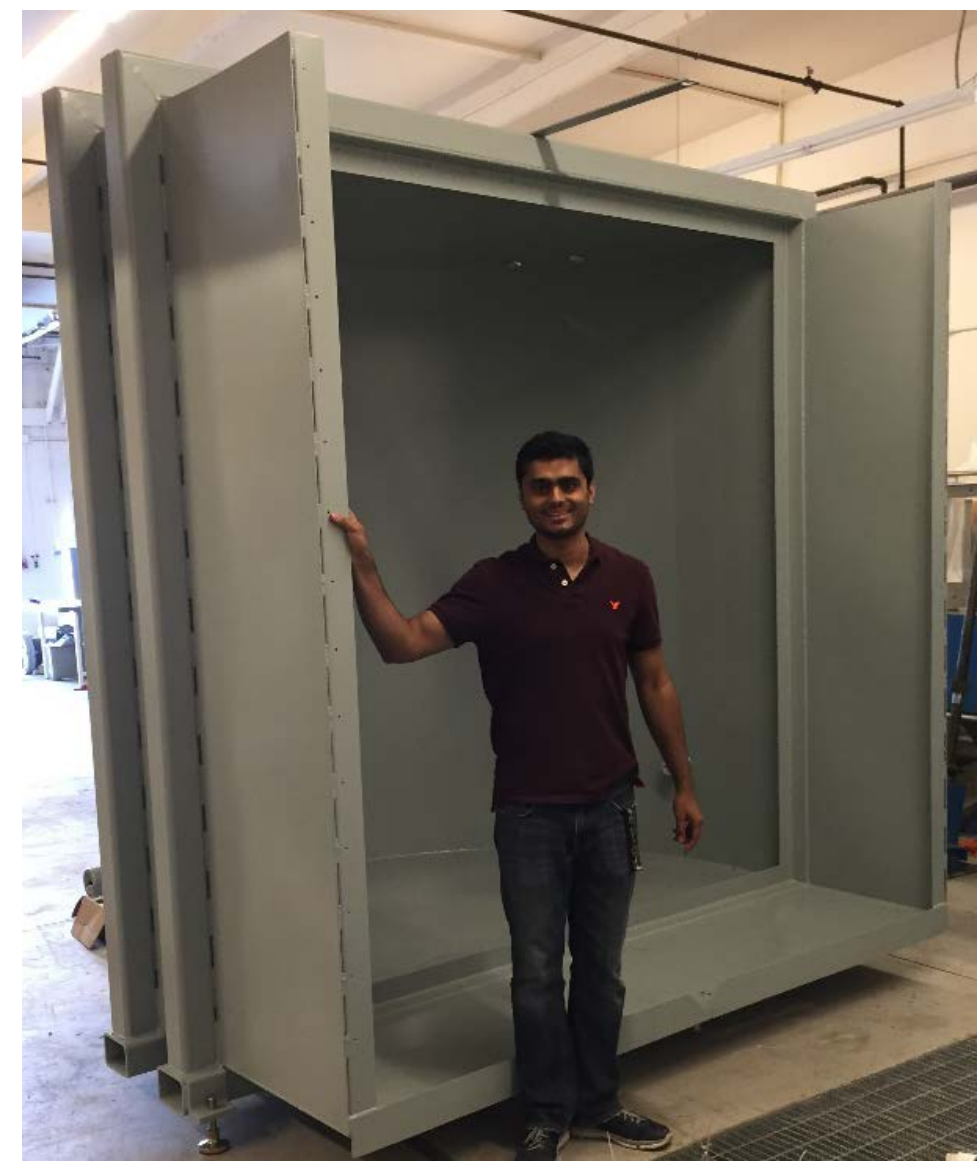

Figure 4. Portal Evaluation Tank.

The PET is connected to an 8000-gallon water reservoir, a $60 \mathrm{HP}$ pump, and a 5 HP pump. The $60 \mathrm{HP}$ pump is connected using 12 in. PVC piping and the 5 HP pump is connected through 3 in. PVC piping. The pipe and valve arrangement for the PET, shown in Figure 5, allows multiple flow settings and pressurization of the PET. 


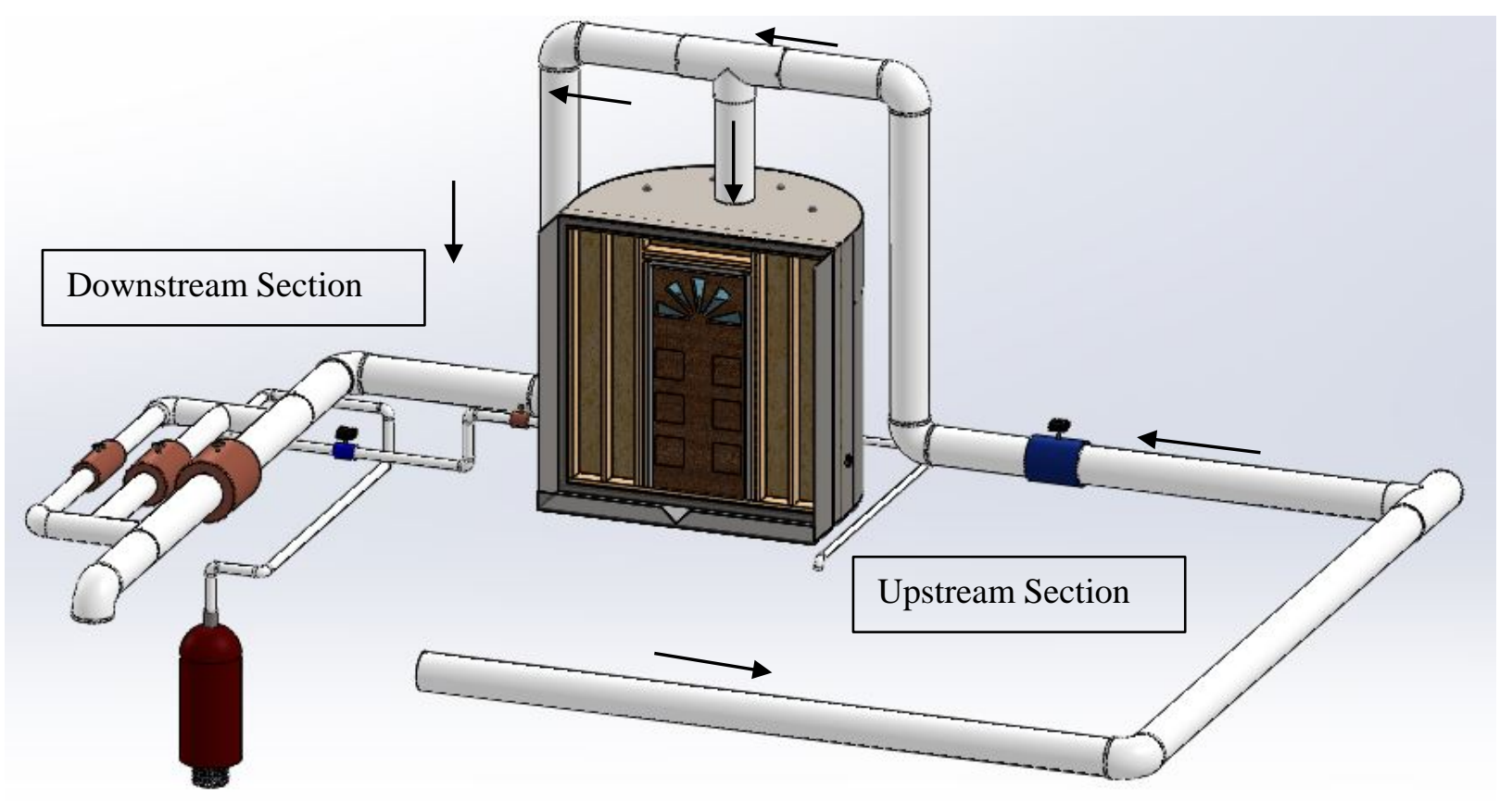

Figure 5. PET piping arrangement.

The piping section that goes from the 60 HP pump to the PET is called the 'Upstream section', it is connected to a return line, that directs the water flow back into the reservoir; and contains two electromagnetic flowmeters, a 12 in. and an 8 in.; butterfly valves are used to direct the water flow to each flowmeter. At the top of the PET, the upstream piping is connected to a 'Tee Joint', some of the water flows into the PET, while the rest flows back to the reservoir, through the 'Downstream Section'. This section consists of two flowmeters of the same size as the ones in the upstream section, it contains 3 distinct piping lines, of sizes of 12 in; 8 in. and 6 in; each line contains a butterfly valve used for two functions; the first one is to adjust the water flow into the tank while it is filling with water, and the second is to be used in conjunction with the valve in the return line, to adjust the water pressure and flowrate in the system when the tank is full with water. Inside the PET, a continuation of the 12 in. pipe goes from the top to the bottom of the tank (Figure 6); this pipe section has holes drilled in it to evenly distribute the water flow into the tank. The overall PET P\&ID is shown in Figure 7. 


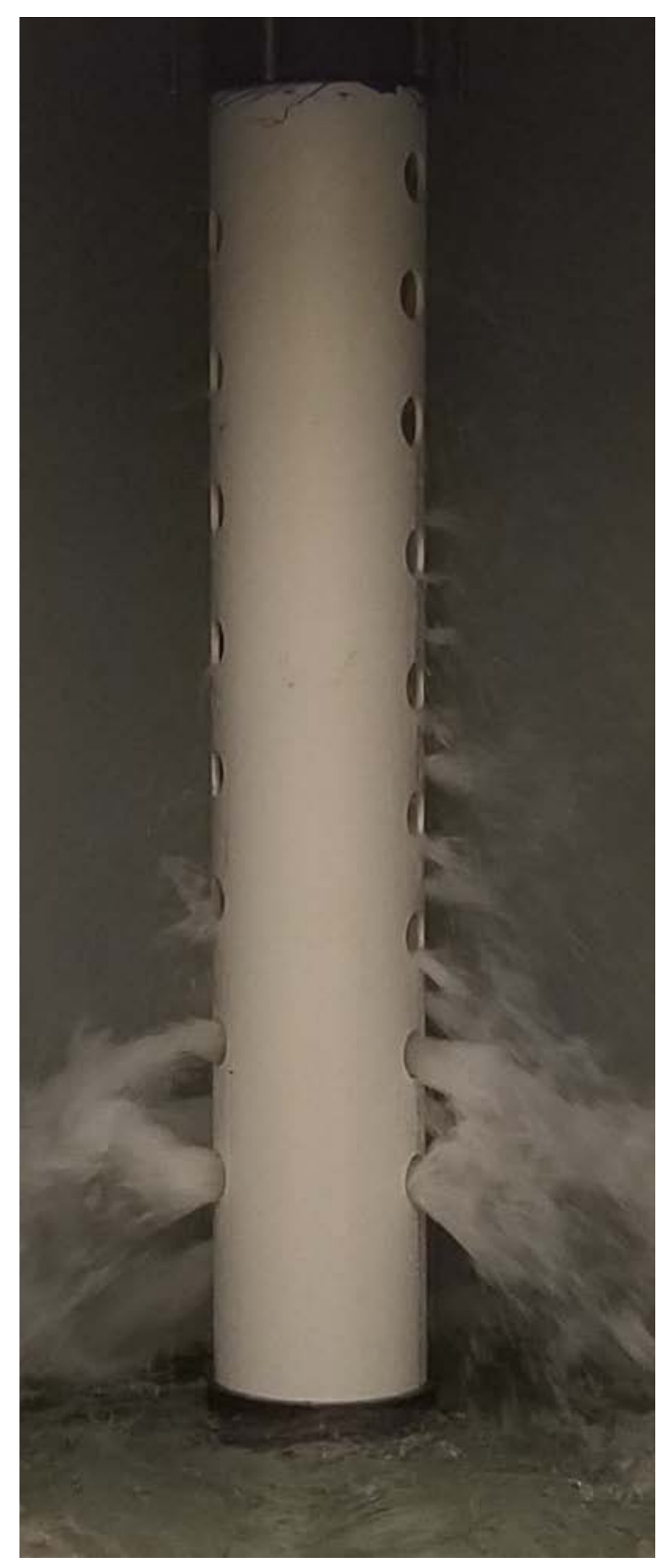

Figure 6. Pipe segment inside the PET. 


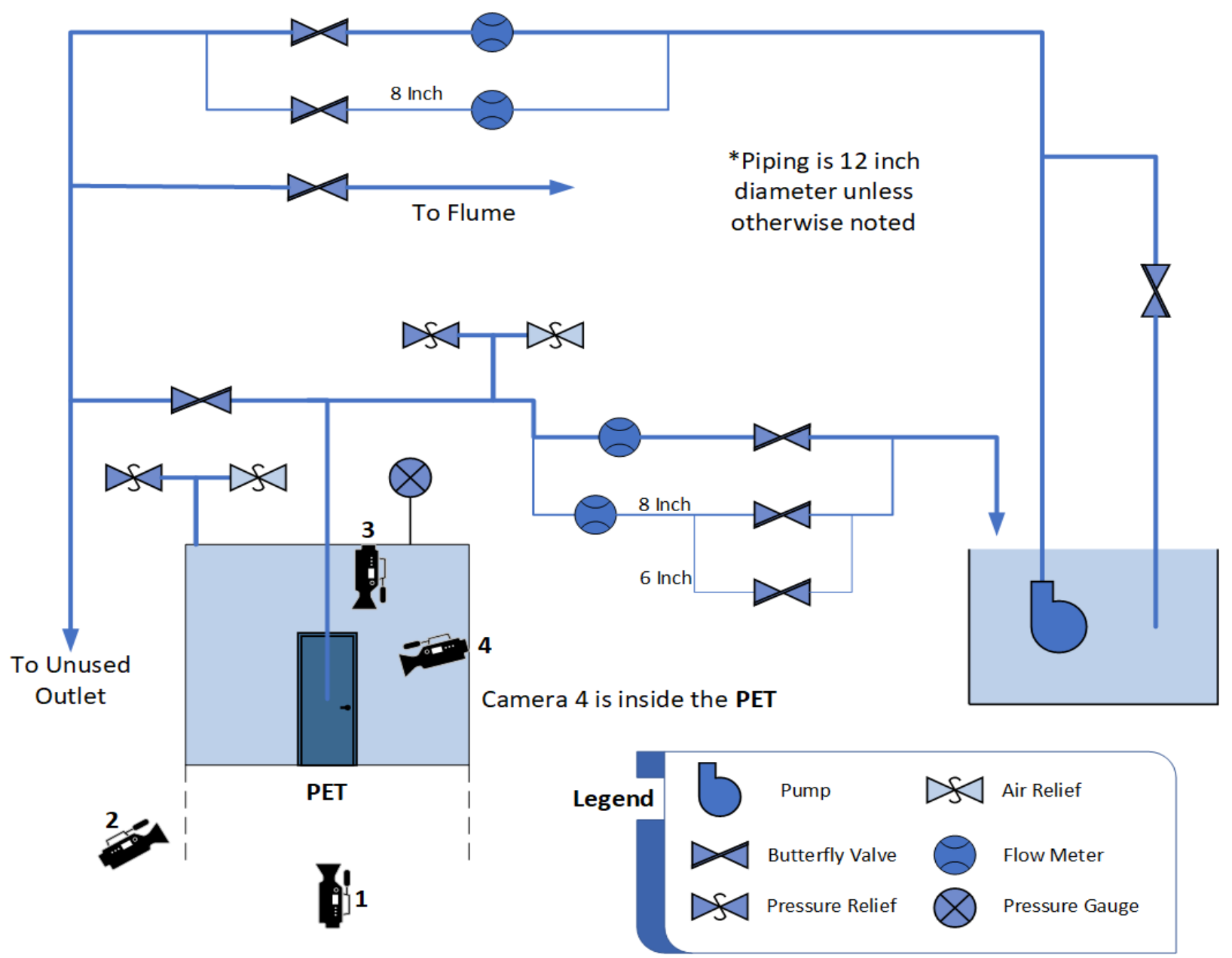

Figure 7. PET piping and instrumentation diagram.

Before performing experiments using the upgraded PET, system simulations were performed in Flow3D to understand how they system behaves. In particular, it is known that when a system is being filled, a brief pressure spike may occur at the time the system reaches the filled status. It is necessary to understand the pressure spike to ensure all components within the system have adequate capacity to accommodate the pressure spike.

In the first Flow 3D simulation, the flow from the pump is discharged directly into the PET. This was devised as a worst-case scenario. The initial conditions are the following: fluid depth at $\mathrm{t}=0$ seconds is $7.5 \mathrm{ft}$; water flowrate(Q) is $4500 \mathrm{gpm}$. As seen in Figure 8, the pressure rises almost linearly while the tank is filling, but at the moment the tank is filled, a pressure surge occurs, creating a maximum pressure of 662.09 psf. which translates to 4.60 psi. 


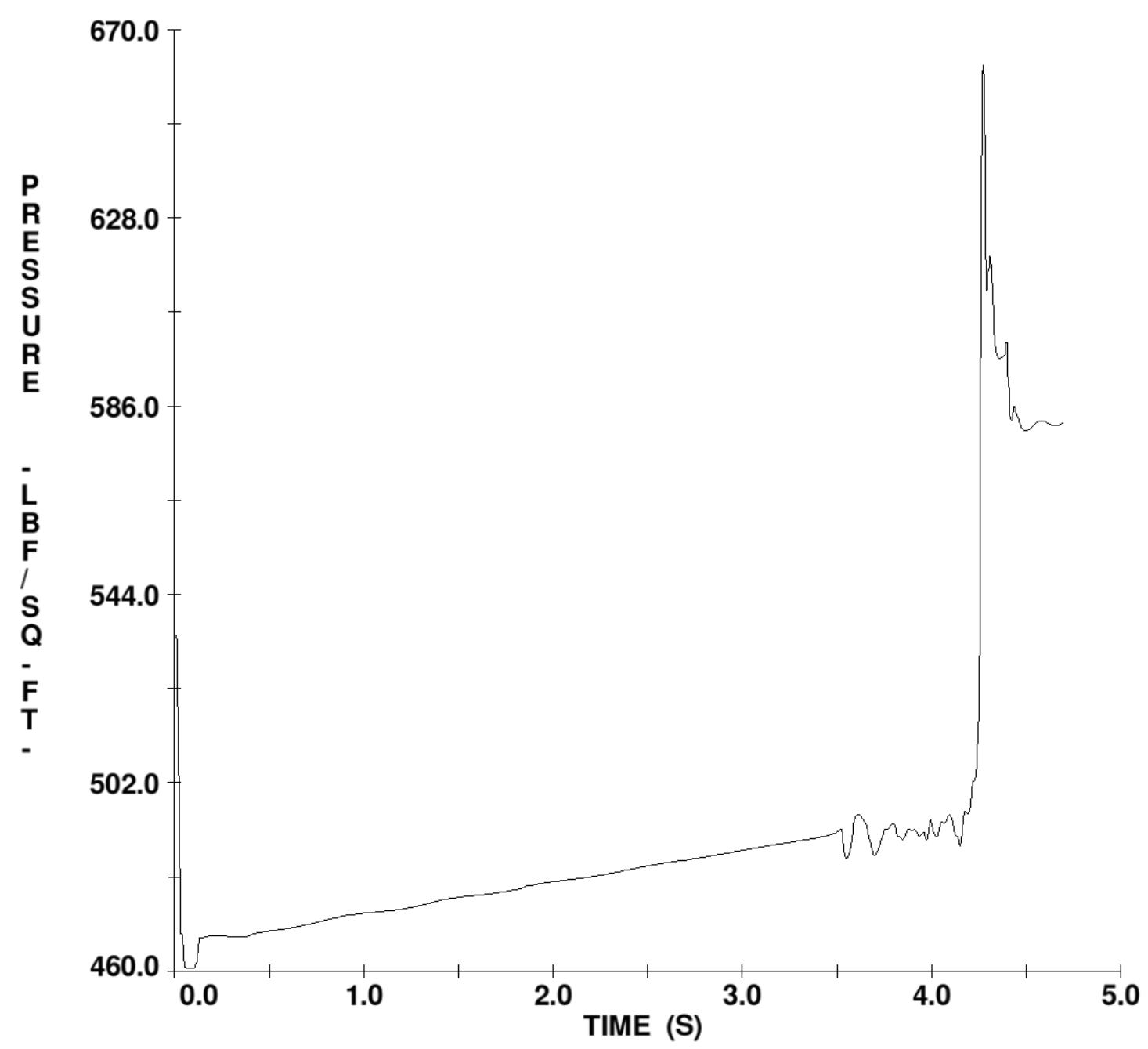

Figure 8. Flow3D simulation of PET pressure.

In the second simulation, the t-joint at the top of the PET was included allowing some of the water to divert into the PET and some of the water returning to the supply reservoir. The simulation initial conditions are as follows: fluid depth in the PET at $\mathrm{t}=0$ seconds is $7.75 \mathrm{ft}$; water flow(Q) is 4,500 gallons per minute. Figure 9 shows small pressure variations occurring during the filling process of the tank. A pressure spike is not present given the lower water flowrate entering the PET. The gradual pressure increase at the end represents the filling of the lower section of the T-joint. From the simulations results, the pressure spike that occurs at the moment the PET fills is within the system capacity. Even if the PET was filled with the 60 HP pump running at full capacity, the pressure spike would not jeopardize the system. 


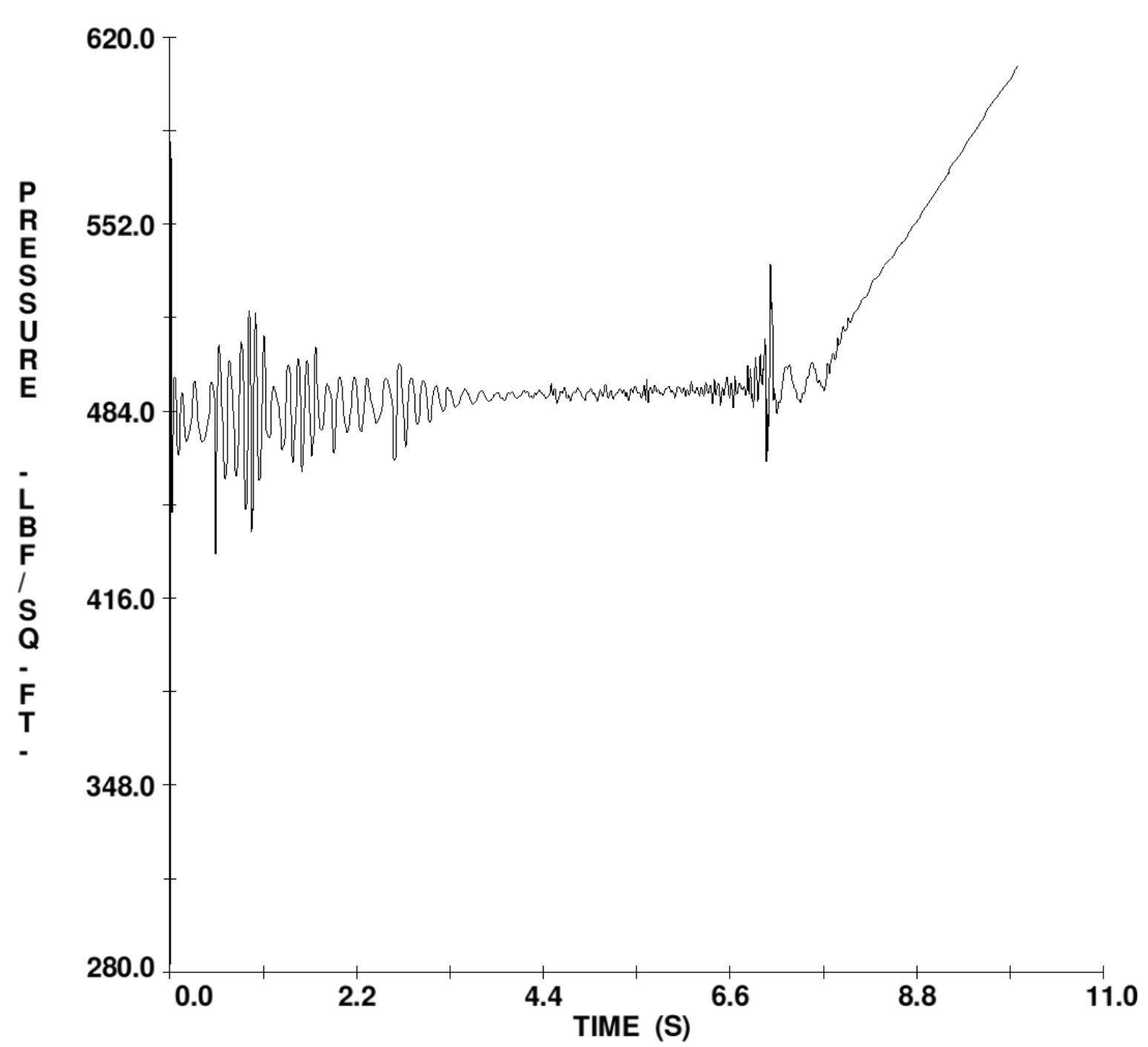

Figure 9. Flow3D PET pressure with return open.

Two types of experiments have been performed with the upgraded PET; hollow core door experiments and steel door experiments. The two first experiments with the upgraded PET were performed on hollow-core doors serving as trials and allowing the team to get acquainted with the equipment and new capabilities.

The two experiments (identified as Experiment \#6 and \#7) were run through the new 12 in. lines; the pump recirculation valve was left open for Experiment \#6, while it was closed for the following experiment. The valves that directed the flow through the $12 \mathrm{in}$. lines were the only ones open, all the other valves were closed. The 12 in. upstream and both downstream flowmeters were connected to a computer using the multi-drop method to connect them through a single HART Modem, PACTware was used, together with the CodeWrights GMBH Hart Communication DTM for the modem, and Microflex Generic 6 DTM for the flowmeters. Two depth sensors were also connected. PET depth results of both hollow-core door experiments are shown in Figure 10. In both experiments, the door was configured to be inward opening which results in increasing water depth causing the door to attempt to seal. Both doors failed catastrophically. The data collected in each experiment was analyzed to produce the following information; water flowrate into the PET, water flowrate out of the PET, and the PET fillrate. The flow measurements for Experiment \#6 are shown in Figure 11. 


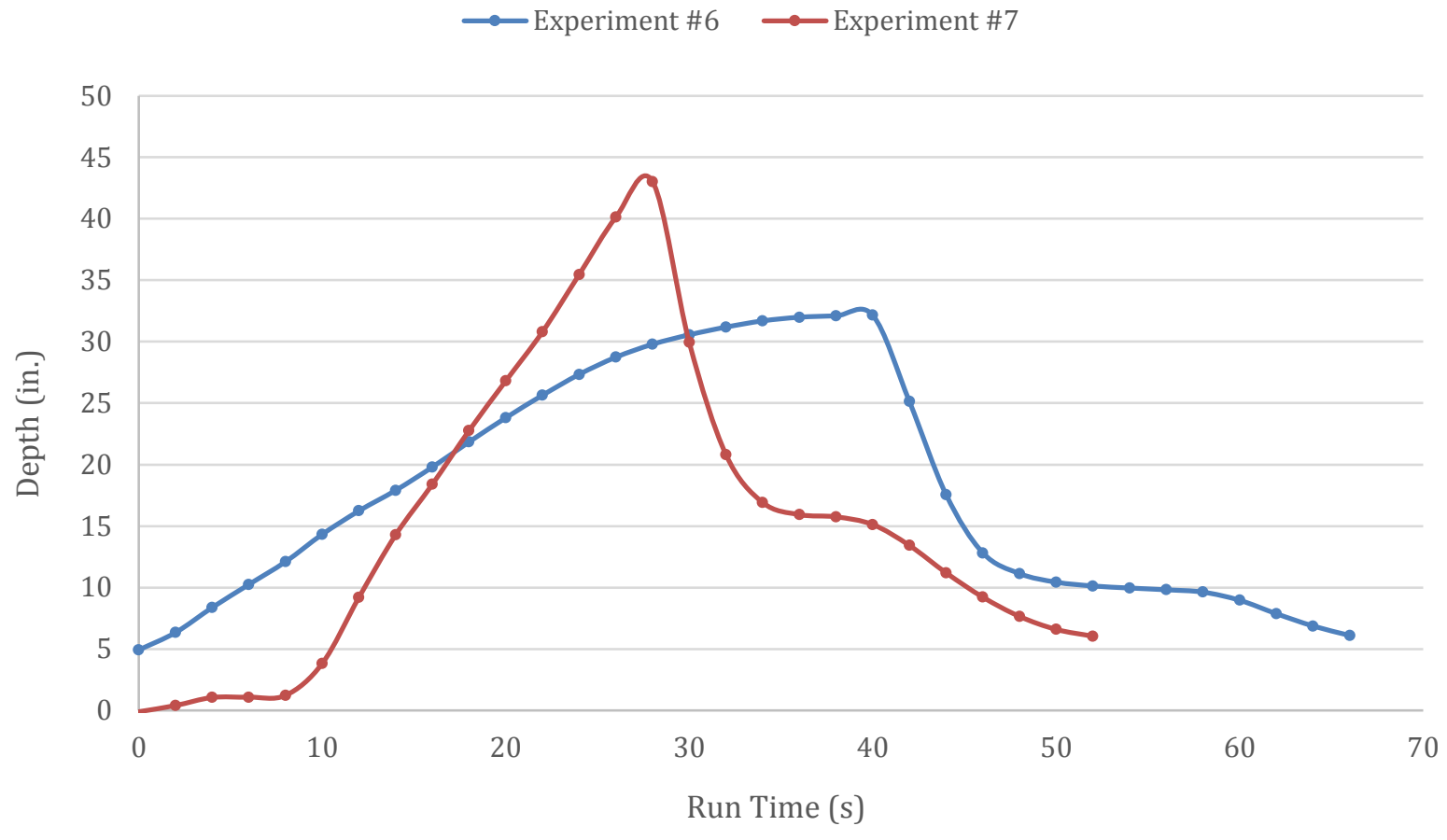

Figure 10. Hollow-core door experiments - PET water depth.

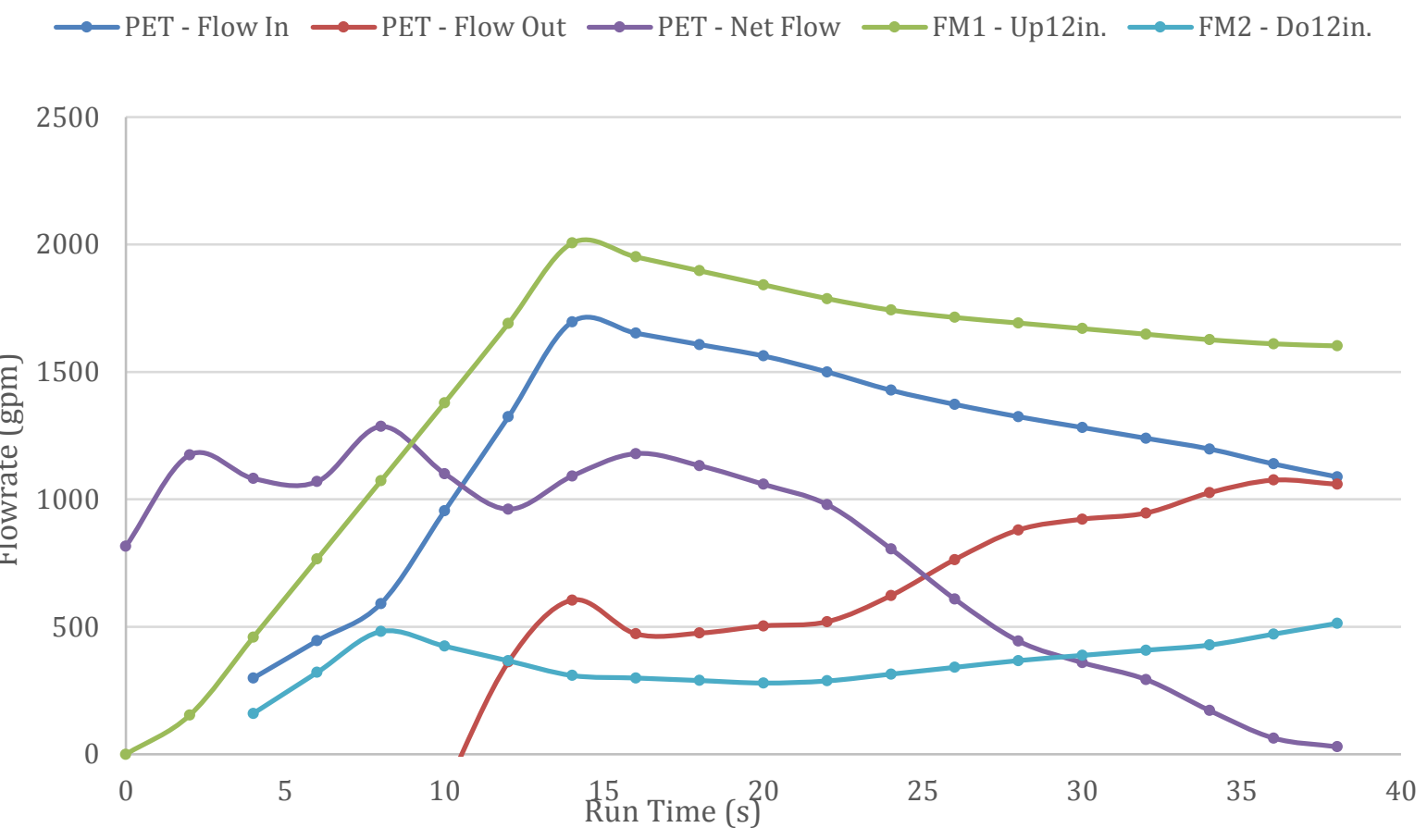

Figure 11. Hollow-Core door experiment \#6 - flow information.

For hollow-core door experiment \#7, the experiment configuration was changed, the pump recirculation valve was closed, this caused the flow through the upstream section to achieve values upwards of 4,000 gpm; 
however, this experiment configuration also resulted in a very short experiment, in which the door ruptured before the flowmeters started reading steady state data.

Unlike experiments with hollow-core doors, steel door experiments were run using only two flowmeters, the $12 \mathrm{in.}$ in the upstream section, and mostly the $12 \mathrm{in.}$ in the downstream section. This was done to reduce the time intervals at which the flowmeters recorded data. The first three steel door experiments were run using the same configuration; the door was configured opening outward and only the door latch was used to keep the door closed. Figure 12 shows the resulting PET water depth. In each experiment the door latch released allowing the door to open, see Figure 13. In the experiments, the recirculation valve was open, as were the valves in the upstream and downstream section of the $12 \mathrm{in.} \mathrm{line.} \mathrm{The} \mathrm{door} \mathrm{was} \mathrm{latched} \mathrm{closed} \mathrm{and} \mathrm{the} \mathrm{deadbolt} \mathrm{was} \mathrm{disengaged.}$ Flow information for these three experiments is provided in Figure 14, Figure 15, and Figure 16.

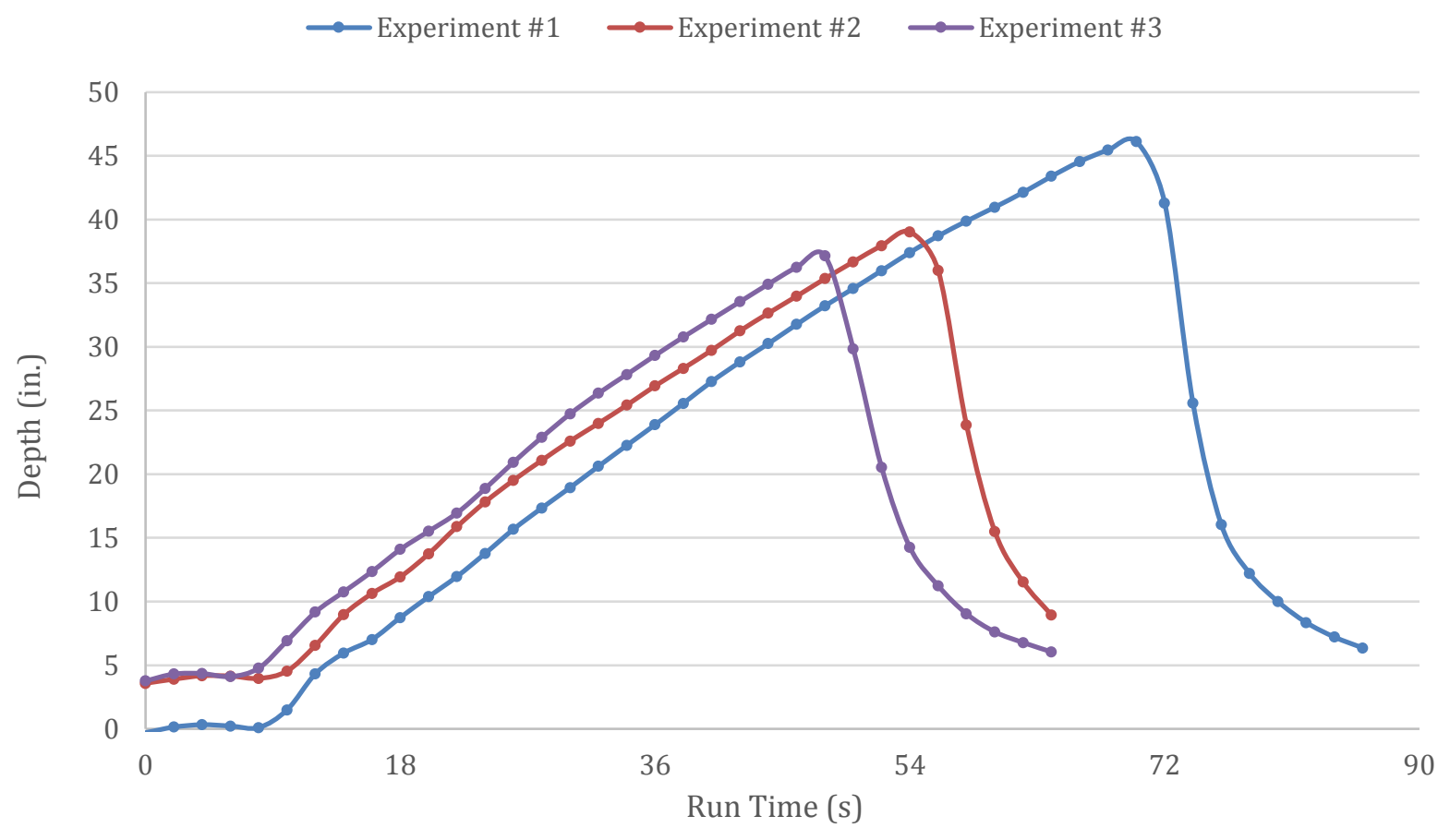

Figure 12. Metal door experiments \#1, \#2 \& \#3 - PET water depth. 


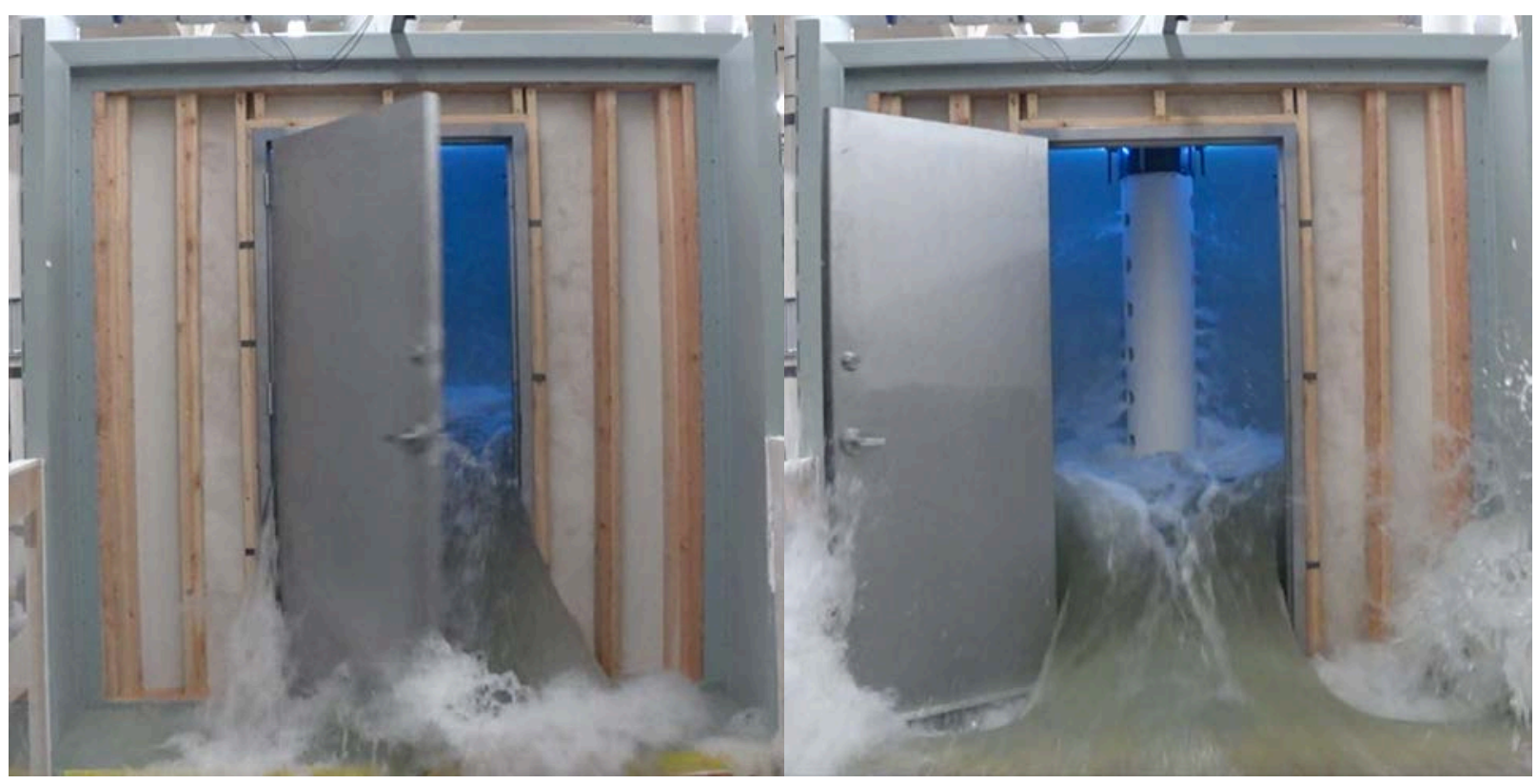

Figure 13. Steel door at the moment of failure.

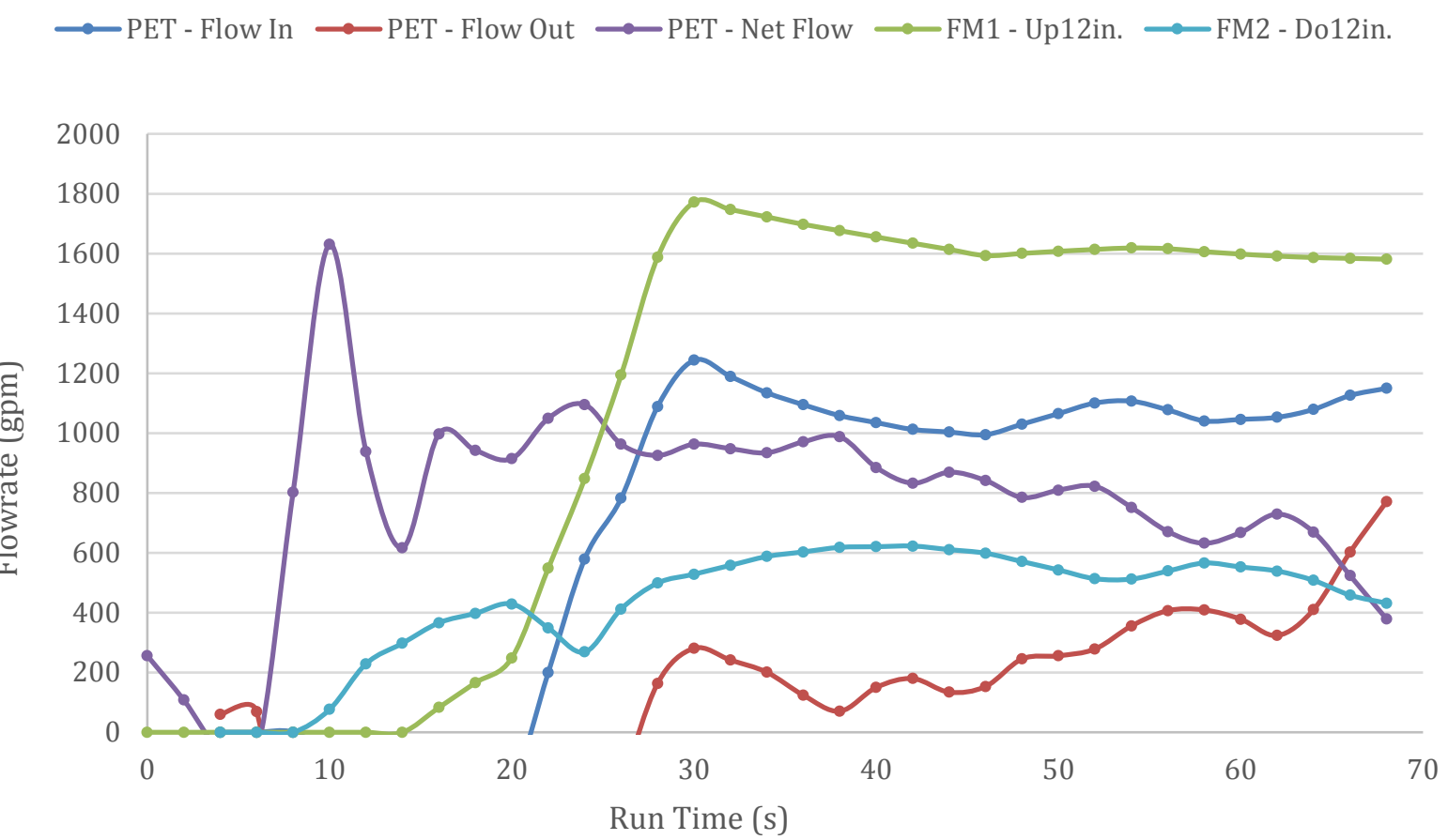

Figure 14. Metal door experiment \#1 - flow information. 
$\longrightarrow$ PET - Flow In $\longrightarrow$ PET - Flow Out $\longrightarrow$ PET - Net Flow $\longrightarrow$ FM1 - Up12in. $\longrightarrow$ FM2 - Do12in.

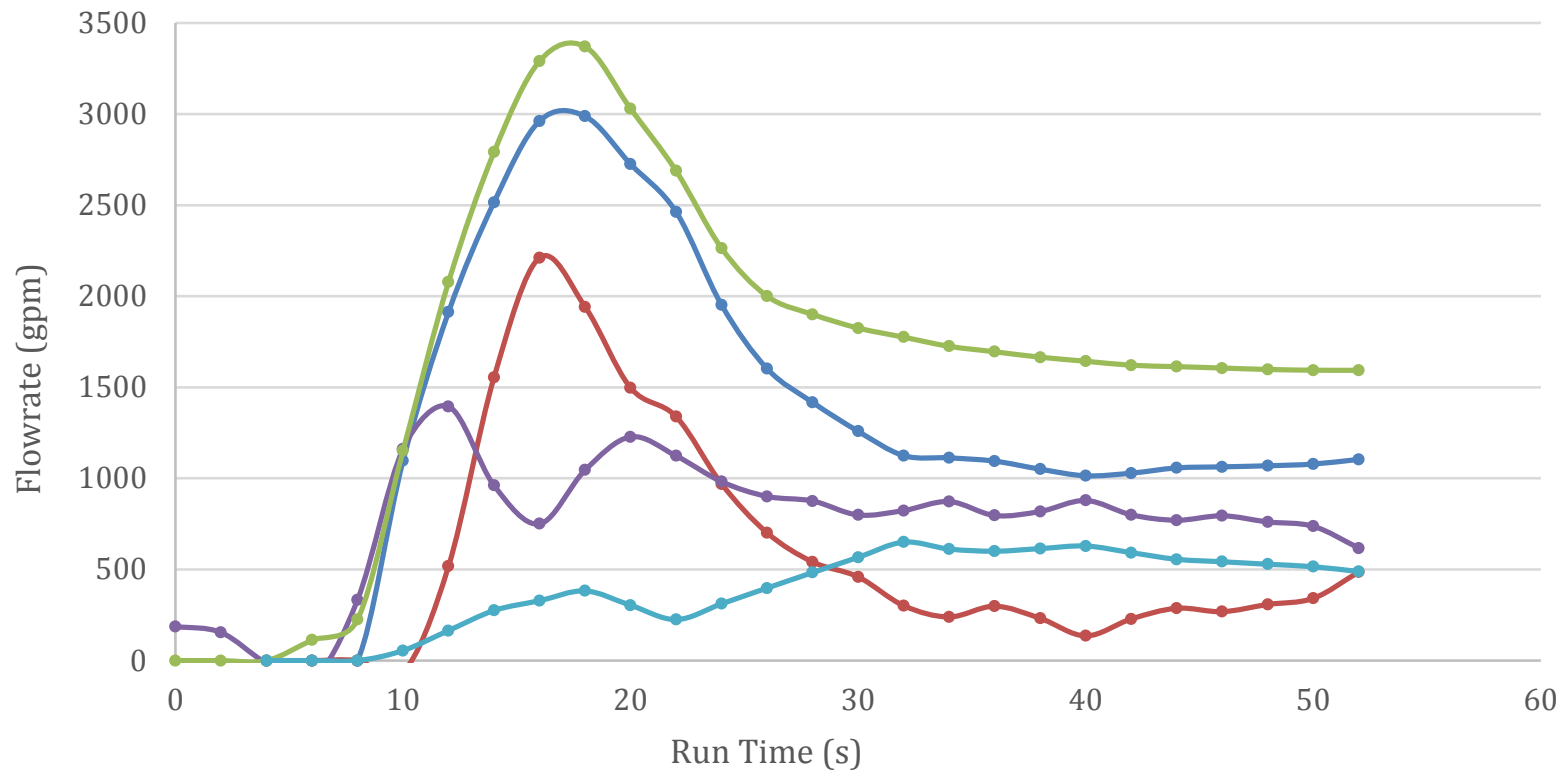

Figure 15. Metal door experiment \#2 - flow information.

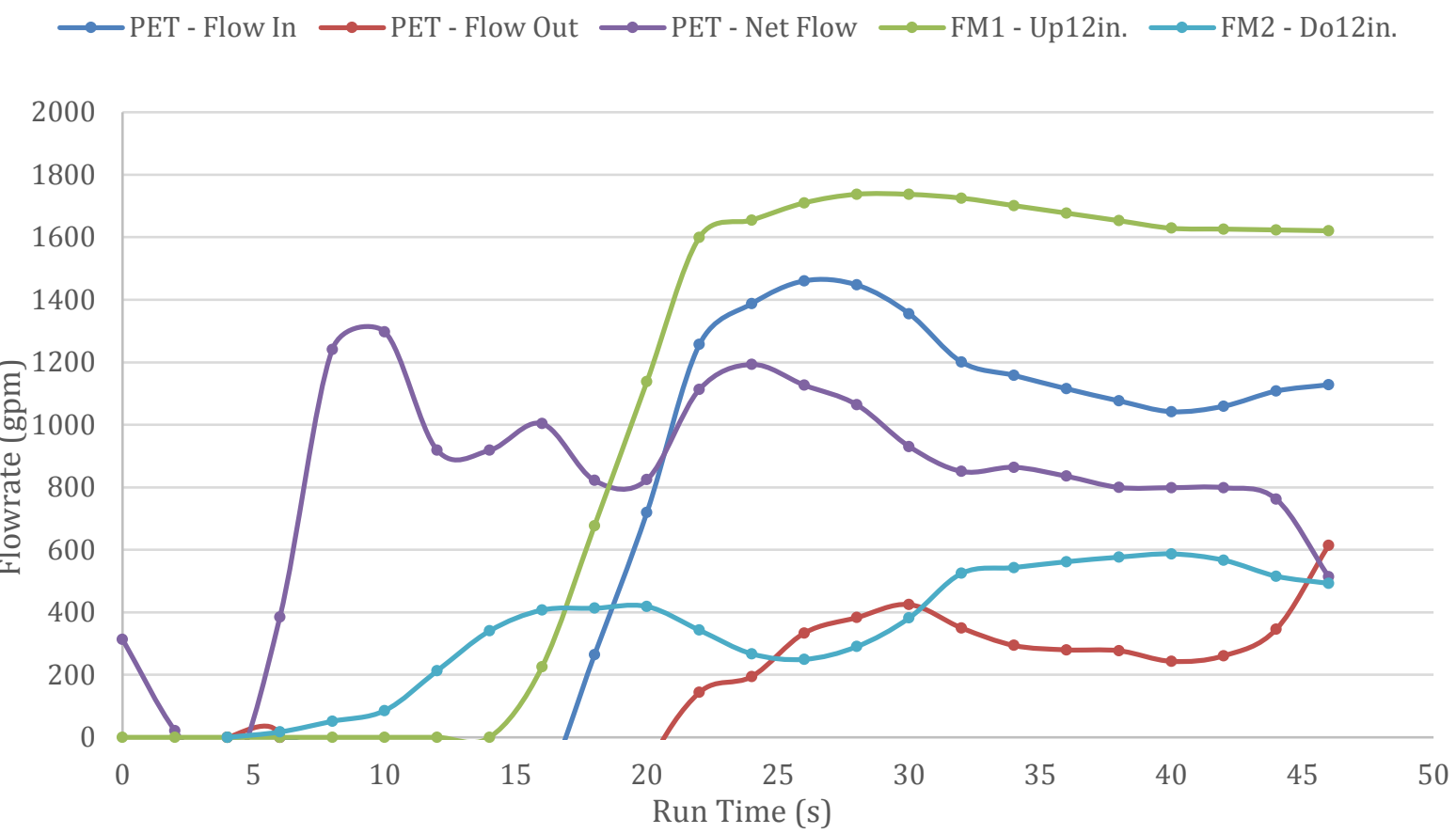

Figure 16. Metal door experiment \#3 - flow information.

The next two experiments were similar to the first three steel door experiments. The main difference was the valve configuration. Experiment \#4 was performed with the recirculation valve open, and the upstream $12 \mathrm{in}$. 
line half-open. Experiment \#5 was performed with the recirculation valve also in the open position, but with the upstream 12 in. valve three-quarters open.

The PET water depth achieved in both experiments was almost identical regardless of the difference in time of the experiments. The difference in time is a product of the lower water flowrate into the PET in experiment \#4, which resulted in a longer time to achieve the water height that caused the door to open. The water depth results achieved for experiments \#4 and \#5 are shown in Figure 17.

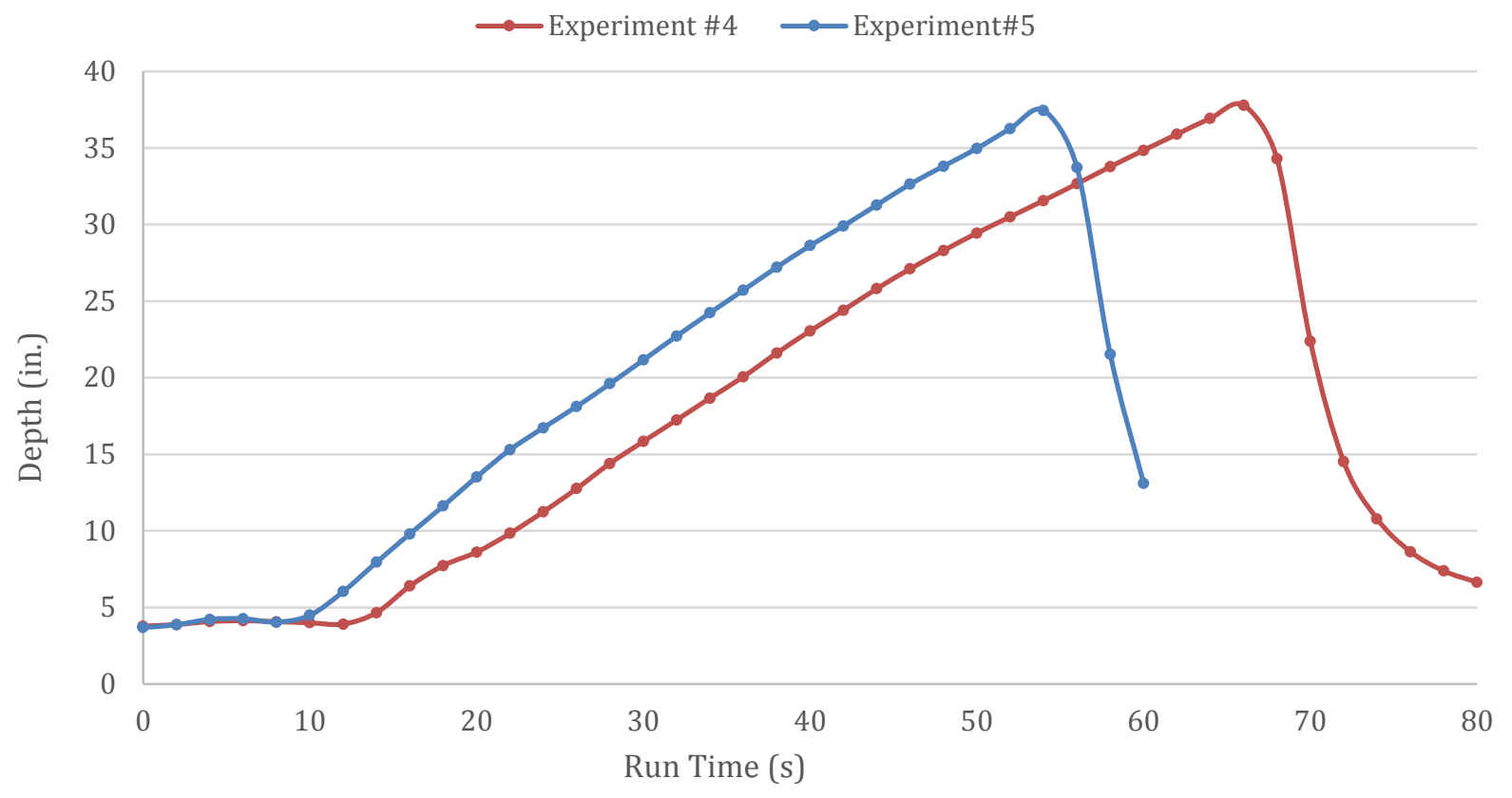

Figure 17. Metal door experiment \#4 \& \#5 - PET water depth.

In experiment \#5 in which the recirculation valve was open the upstream water flowrate through the upstream $12 \mathrm{in}$. flowmeter reached a steady-state value close to 1,700 gpm, while in experiment \#4, the flowrate was always closer to 1,400 gpm. In experiment \#4 the water flowrate into the PET varied between 600 and 900 gpm; while in experiment \#5, with the higher upstream water flow, the flowrate into the PET was also higher, closer to 1,000 gpm. Flow rates for experiments \#4 and \#5 are provided in Figure 18 and Figure 19. 


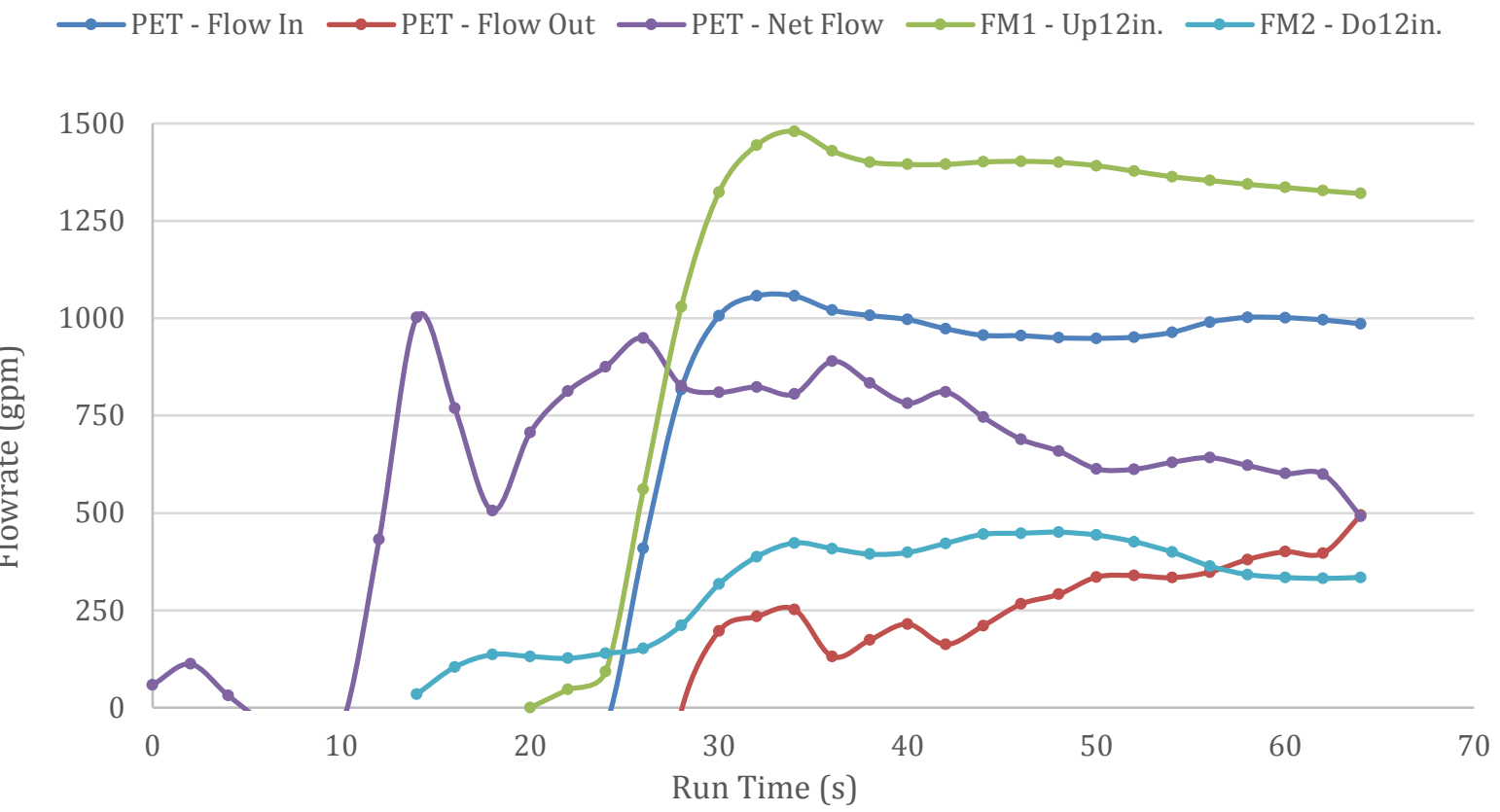

Figure 18. Metal door experiment \#4 - flow information.

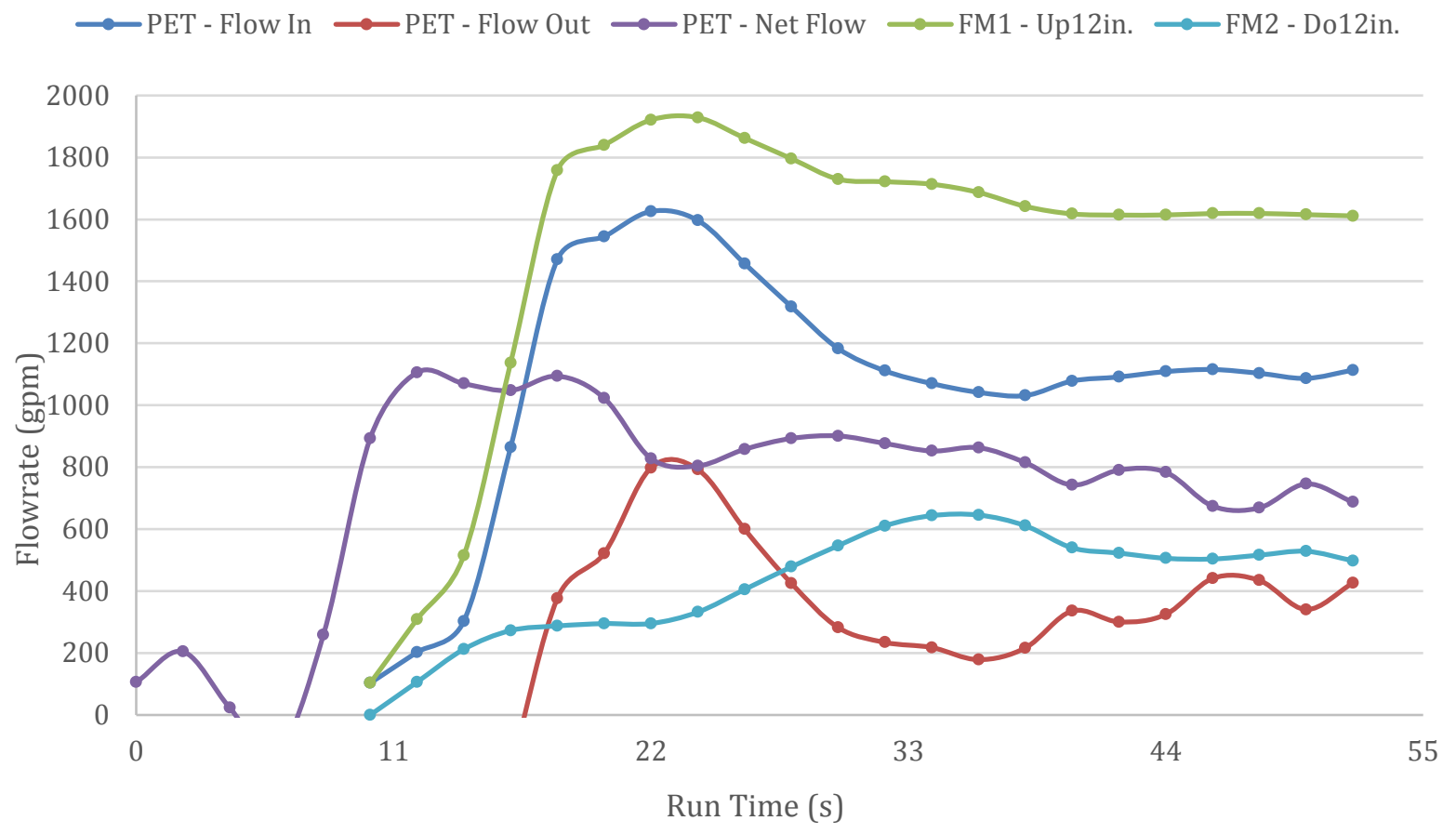

Figure 19. Metal door experiment \#5 - flow information.

The next three experiments were performed with the steel door opening outward, latched and an open deadbolt; the recirculation valve was open, as in the previous two experiments, but this time the upstream 12 in. valve was only open one-quarter of the way. Figure 20 shows the PET water depths achieved during each of these 
experiments. By comparing results from these three experiments, it is possible to conclude that the steel door has a very constant behavior.

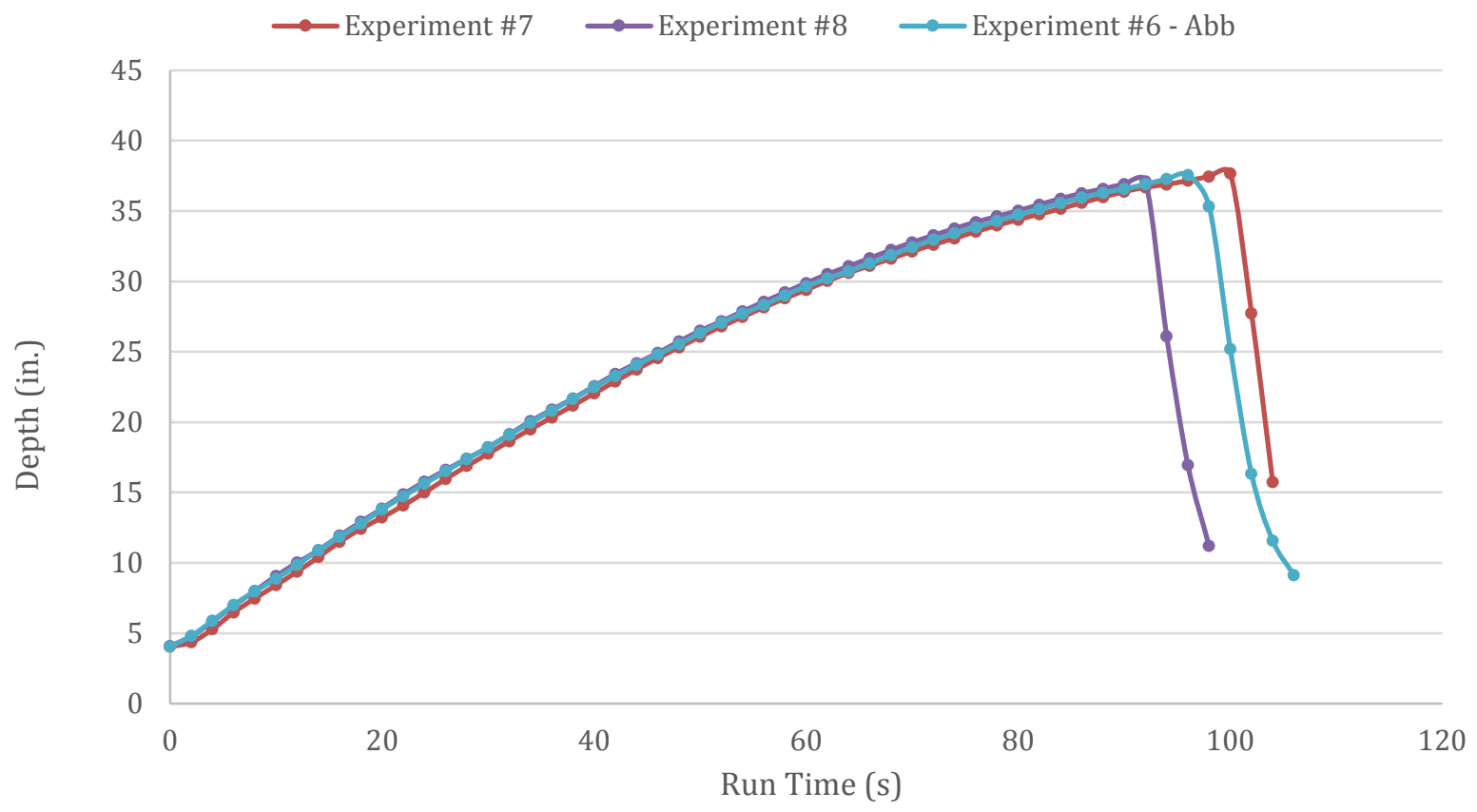

Figure 20. Metal door experiment \#6, \#7 \& \#8 - PET water depth.

The next three experiments, Experiments \#9, \#10 and \#11, were all performed with the deadbolt engaged. The Experiment \#9 configuration had the recirculation valve closed and the upstream $12 \mathrm{in}$. valve half open. As seen in Figure 21, closing the deadbolt produced only a slight increase in the maximum water depth achieved inside the PET, compared to the previous experiments. In experiment \#9 the deadbolt internally failed, Figure 22, rather than shearing. In experiment \#10, the deadbolt bent sufficiently, Figure 23. to allow the door to open.

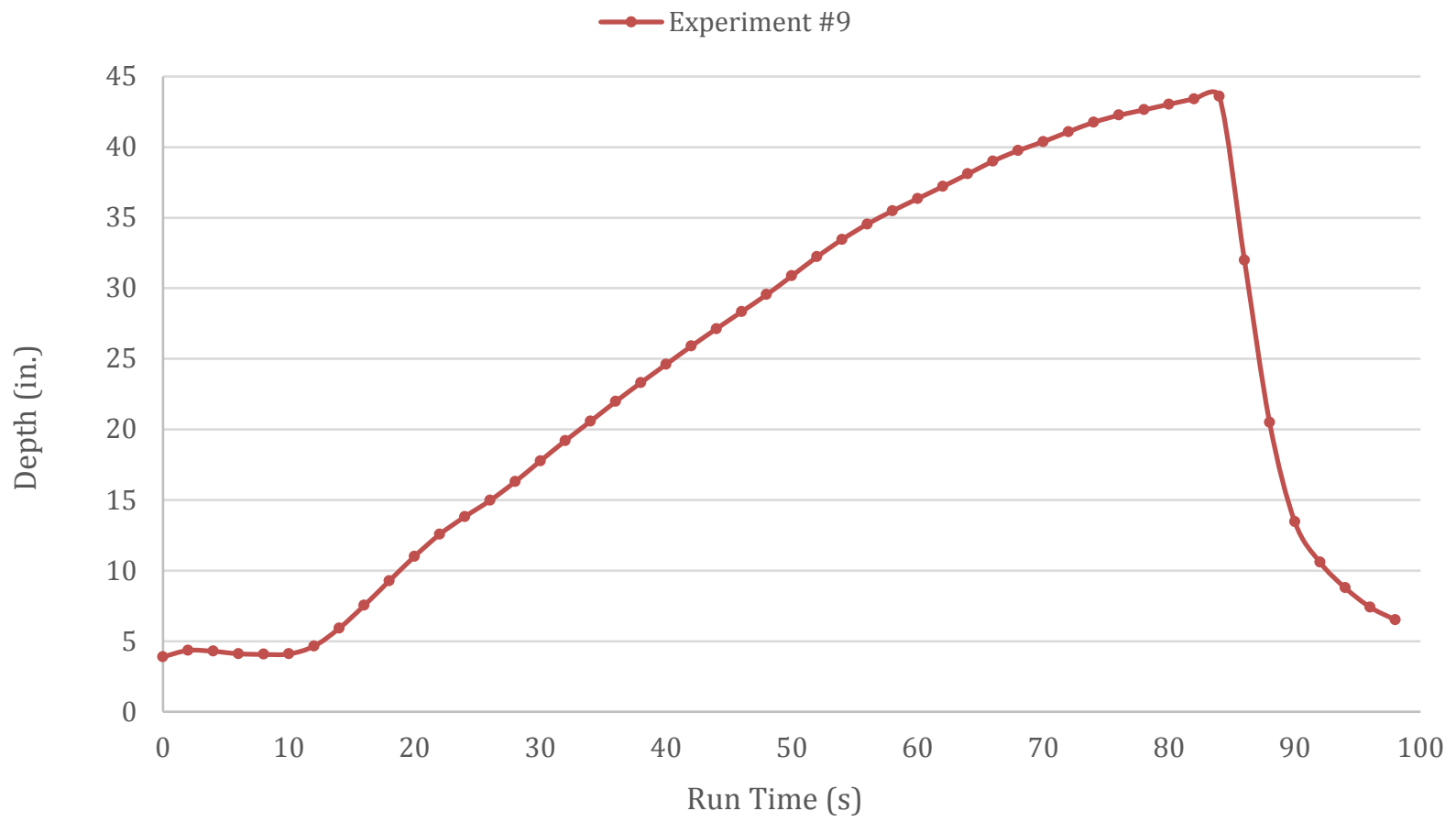


Figure 21. Metal door experiment \#9 - PET water depth.

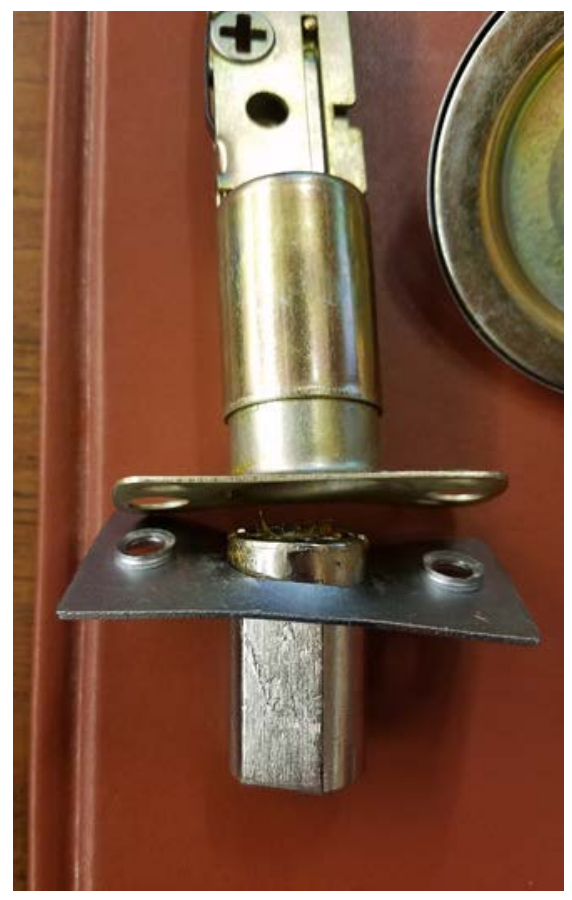

Figure 22 . Deadbolt failure experiment \#9.

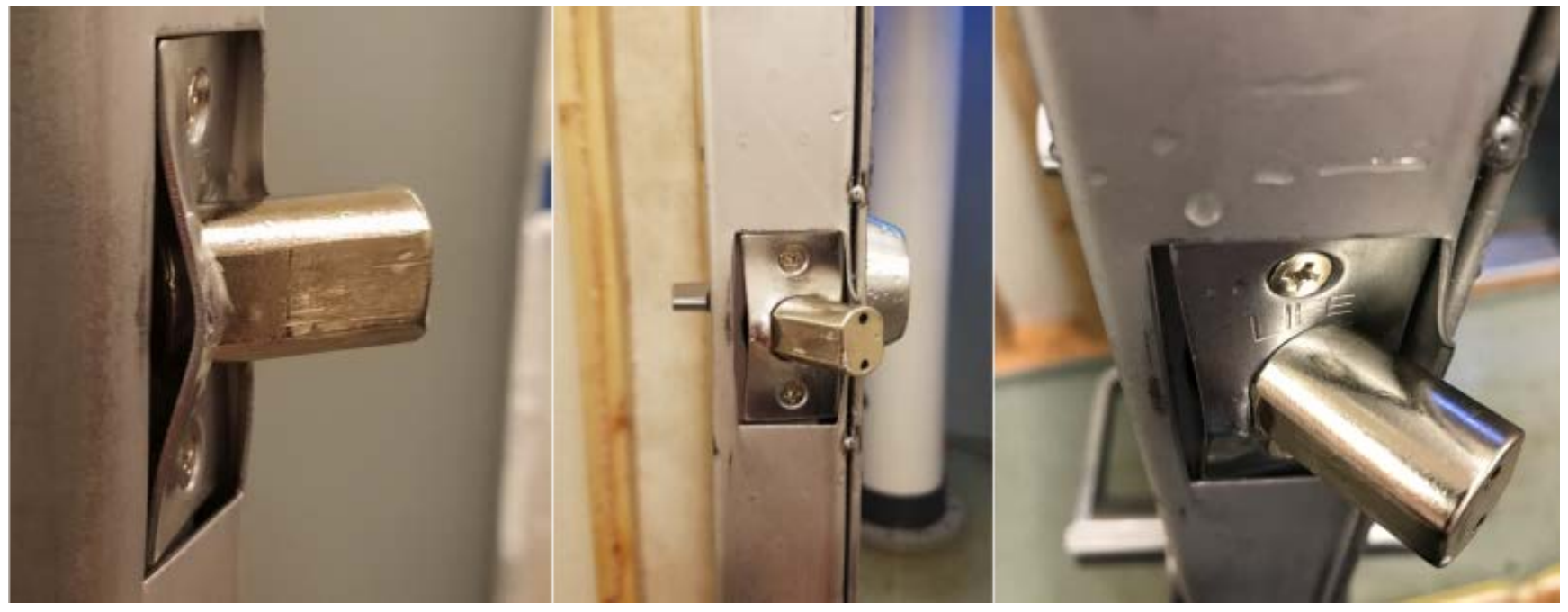

Figure 23. Deadbolt failure experiment \#10.

For experiment \#11 the door was locked in the same way as Experiment\#9 or Experiment\#10, this means both the door latch and deadbolt were locked; however, it is important to note that the deadbolt on this experiment was not the same as in the two previous ones, it was an aftermarket deadbolt acquired from a local hardware store, and this fact may be the cause why the results of this experiment were different than the previous two.

The initial valve configuration for this experiment consisted in having the recirculation valve fully closed, the upstream 12 in. valve one-quarter-way open, and the downstream 8 in. valve fully open (note that in previous experiments the downstream 12 in. valve was open and the 8 in. one closed). As in Experiment \#10, the configuration was not kept constant through the experiment, with the initial valve configuration, water depth inside 
the PET stabilized at 30 in. The upstream 12 in. valve was then opened fully and the downstream 8 in. valve was progressively closed, the results in the flow conditions for the valve movements can be seen in Figure 24 .

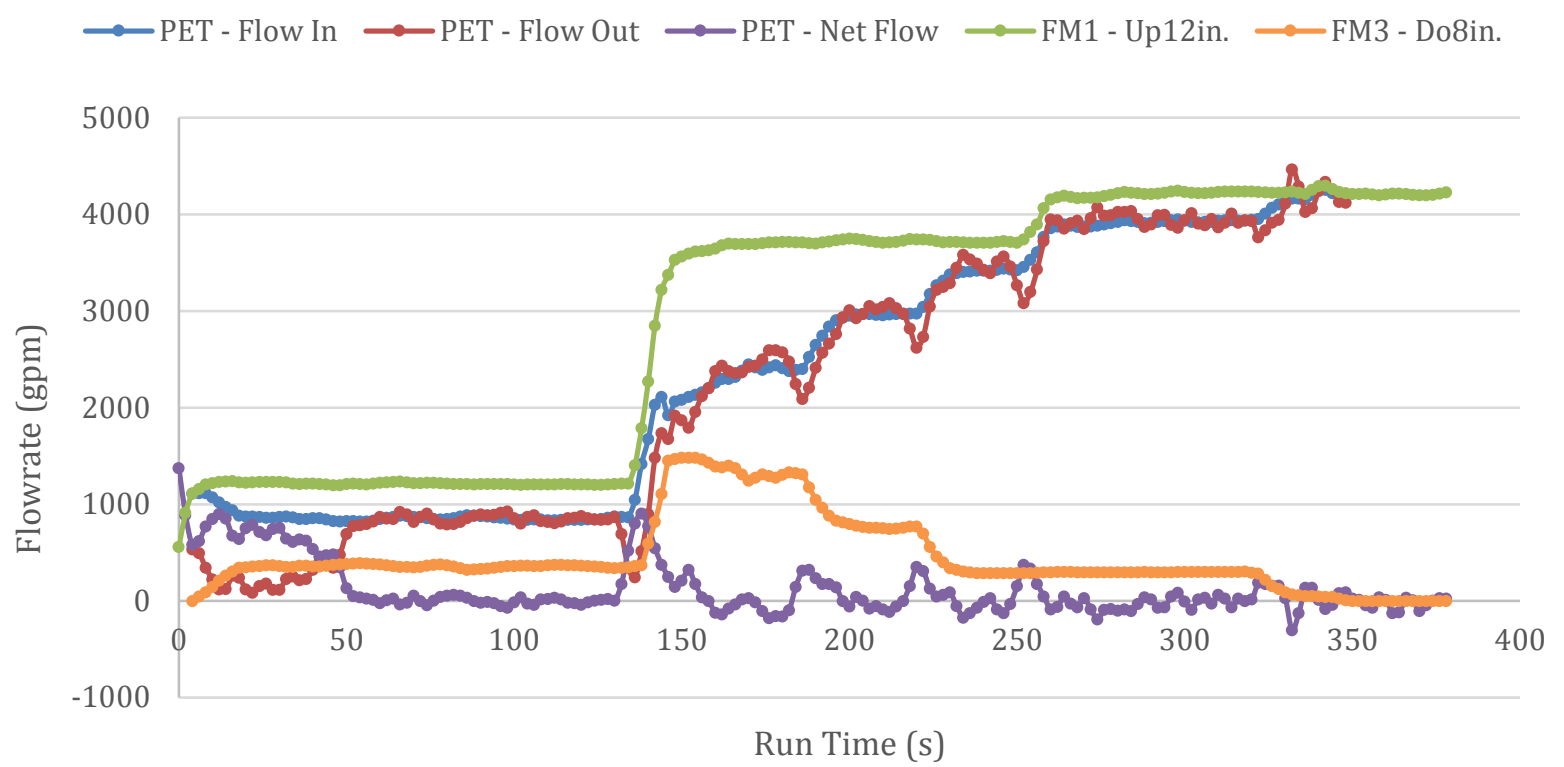

Figure 24. Metal door experiment \#11 - flow information

After closing the downstream 8 in. valve though, the experiment conditions were the same ones as in Experiment\#10 after the downstream 12 in. valve was closed, the full flow from the pump was going into the PET; the results from Experiment \#11 were different than those from Experiment\#10. During Experiment \#11 the flow into and out of the PET reached a constant value, even when all the water from the pump was directed towards the PET. This happened because during this experiment the door suffered a permanent bend at the level of the lock, see Figure 25. 


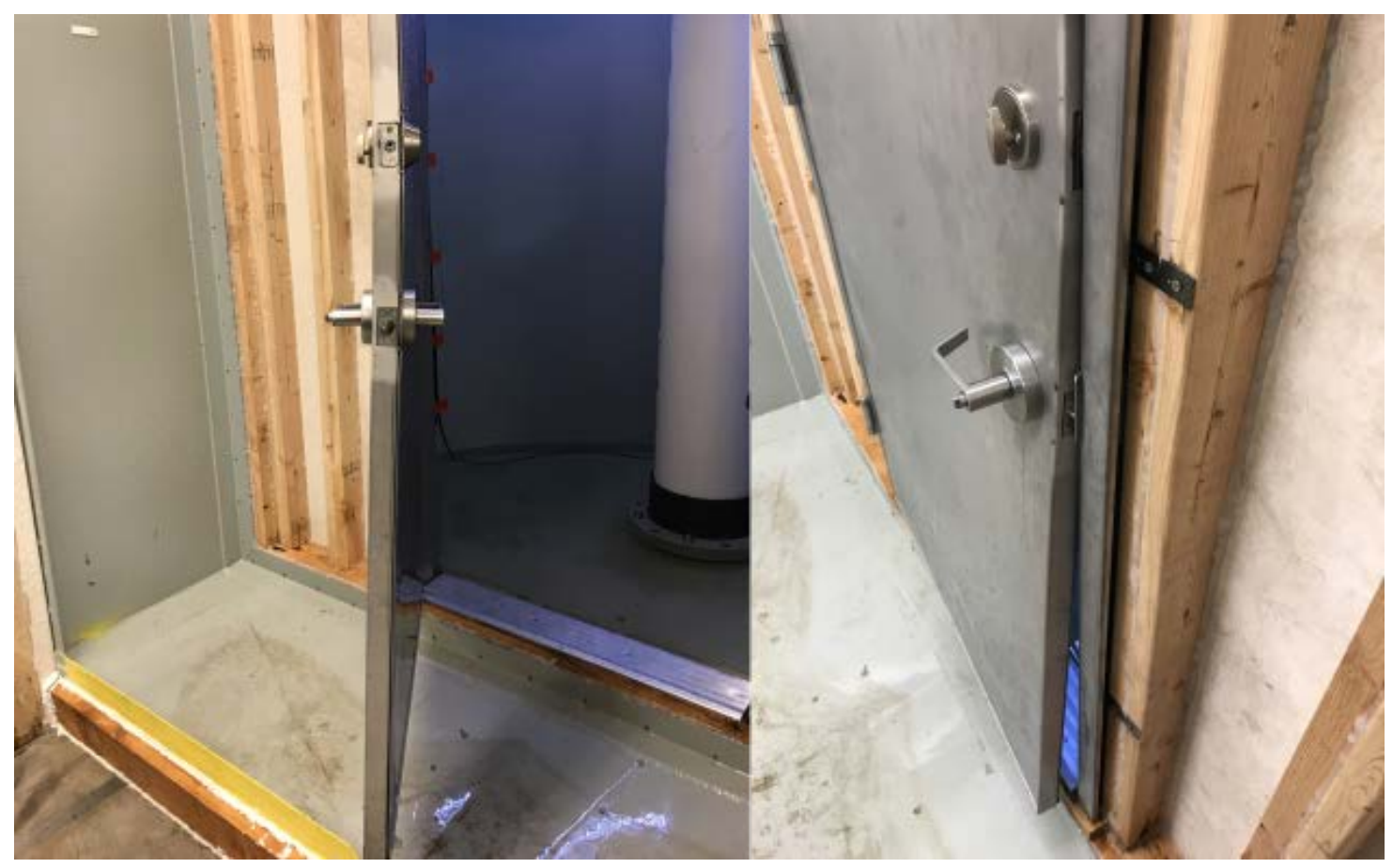

Figure 25. Experiment \#11 - post-experiment door damage.

One of the causes of this unexpected results is the different brand of deadbolt used for the experiment, which can be seen in Figure 26. The bolt itself is longer than OEM lock, and the whole deadbolt system was assembled differently.

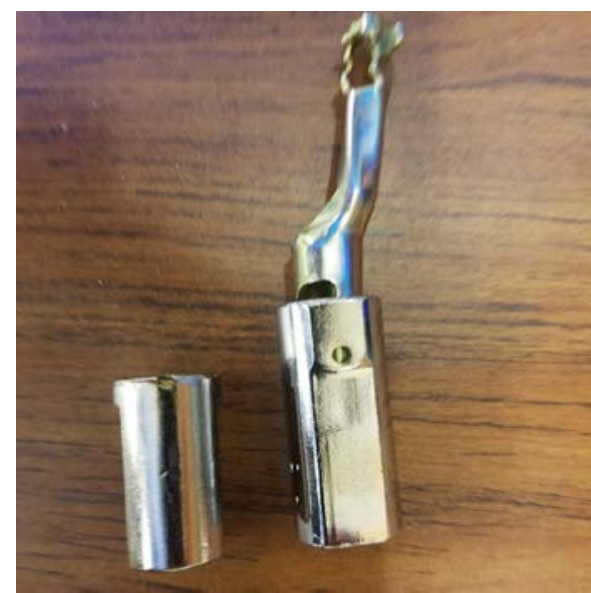

Figure 26. Deadbolt comparison - experiments \#9 \#10 (left-typical), experiment \#11 (right).

Experiment \#12 was performed with a similar initial valve configuration as Experiments \#4, \#5 and \#6. The recirculation valve was open, the upstream 12 in. valve was one quarter open, and the downstream 8 in. valve was also open. Note than in the previously mentioned experiments, the downstream 8 in. valve was closed, while the downstream 12 in. valve was open. This experiment served two purposes. The first was to determine whether the ' 0 gpm' reading of the downstream 12 in. flowmeter for Experiments \#4, \#5 \& \#6 was due to extremely low flows. The second objective was to confirm the water depth achieved in Experiment \#1, which was higher than all other experiments performed with the open deadbolt since the door and latch used for this experiment were new and of the same model as in Experiment \#1. 
The results showing the water depth achieved in this experiment are shown in Figure 27, and the flowrate analysis is shown in Figure 28. The spike at the end of both figures corresponds to the change of the upstream 12 in. valve to half-way open. The deepest water level in Experiment \#12 corresponds to $44.5 \mathrm{in}$, which is 3.6\% lower than that of Experiment \#1, 46.1 in; but 19\% higher than the depth achieved from Experiment \#2 through Experiment \#8. This helps confirm the water depth achieved in Experiment \#1 was related to it being the first experiment rather than an anomaly; although further testing with the same latch and door could be used to further prove this point.

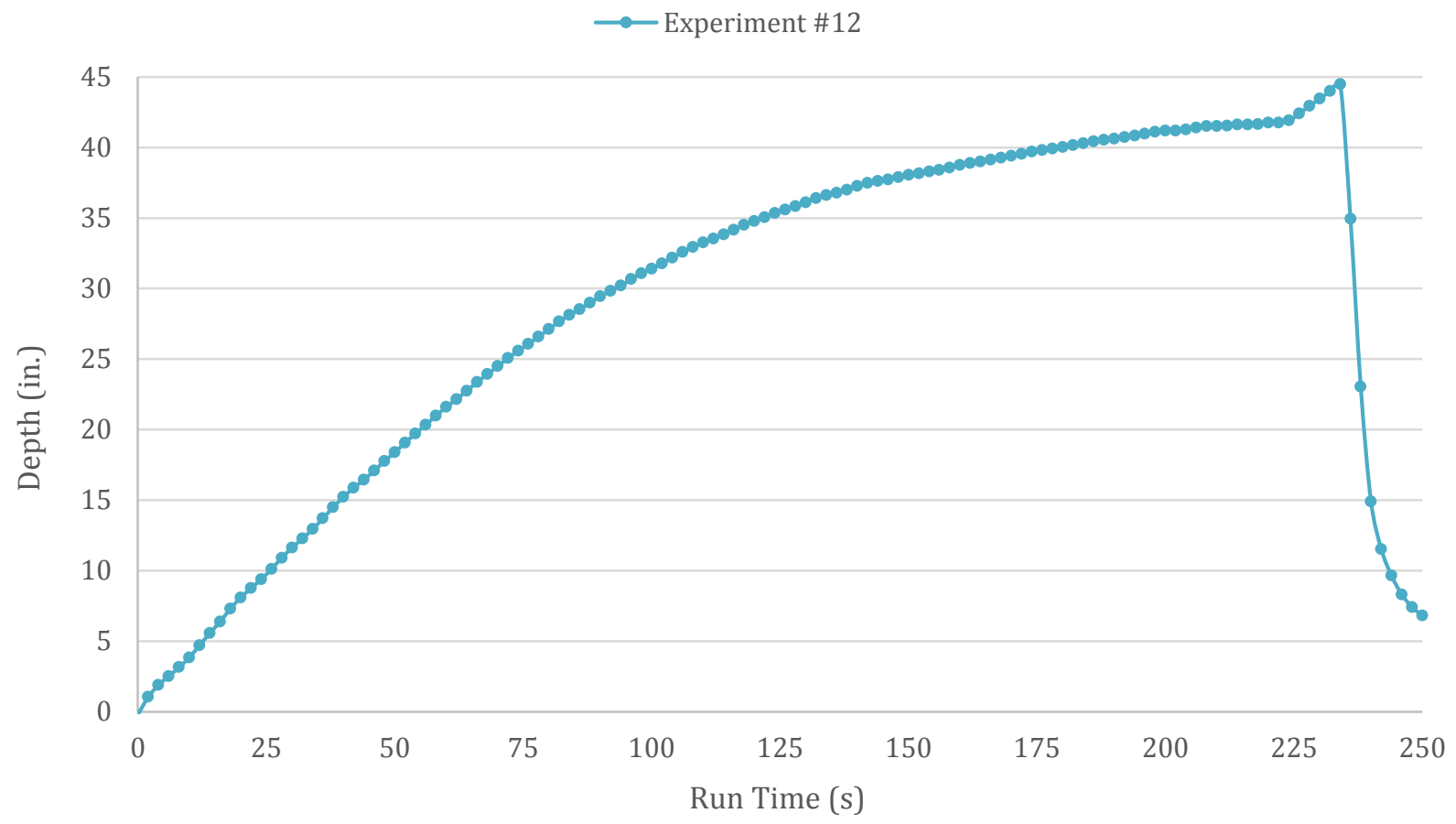

Figure 27. Experiment \#12 - PET water height.

One of the trendlines in Figure 28 shows the readings produced by the 8 in. downstream flowmeter. It can be observed how the readings are ' 0 gpm' for duration of the experiment while the upstream $12 \mathrm{in}$. valve was open one quarter of the way. This can be used to assert than in this configuration, there is either no water flowing through the downstream section of the PET or the flowrate through this section is below 10 gpm, which is the cutoff flowrate for the 8 in. flowmeter. 


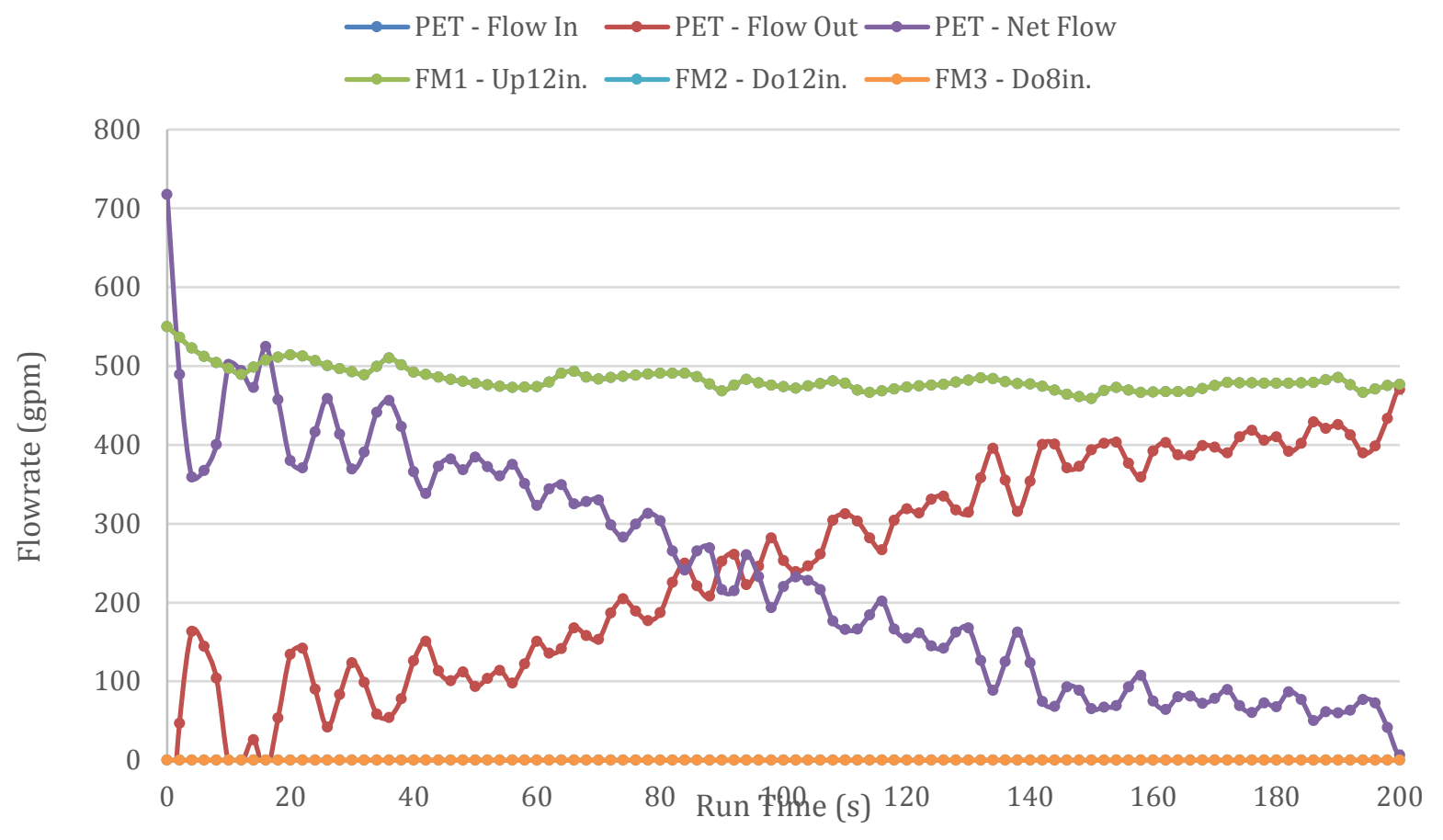

Figure 28. Metal door experiment \#12 - flow information.

Testing involving inward opening steel doors will occur next in the PET followed by NPP fire barrier feedthroughs.

\section{PIPE RUPTURE SPRAY PATTERN}

Significant pipe leakage research has been conducted, however, most research addresses how leaks can occur and the probabilities of occurring. The research is primarily concerned with loss-of-coolant accidents (LOCA), and core damage. LOCAs and core damage may be the most important considerations for nuclear power plants, but the possibilities of damage occurring to equipment suffering water damage from a leak have not been well studied. The goal of this section is to address what has been studied in pipe failures relevant to nuclear power plant components, and to demonstrate where the knowledge is lacking. The basis for the experiments will be justified using data compiled from pipe failures in nuclear power plants.

The Swedish Nuclear Power Inspectorate (SKI) performed a study of pipe failure events at United States NPPs from 1961-1995 [1]. The three most important report databases studies were Licensing Event Reports, Abnormal Occurrence Reports, and Reportable Occurrences. In all, this study aggregated 1511 reports of piping failures in the US and created a Microsoft Access database for the events. The study used six different terminologies to define pipe failures. The first term is leak, defined as a limited but finite amount of water that is released, varying from leaks of cubic centimeters per hour to a liter or more a minute. The following term is crack/leak, defined as having finite depths and penetration of the pipe wall to create a leak, and is a subset of leaks. Thirdly, failure, which, in the context of these reports, is a vague term and is not used in any quantifying ways. Several failures in the studied reports, after further assessment of their associated reports by SKI, were reclassified as leaks. The last three categories, rupture, severed, and breakage, are used synonymously and center on failures the size of the cross-section of the pipe to full double-ended guillotine breaks. Each failure in the database is defined by one of these terms to indicate the severity of the damaged piping. 
The following tables show some categorical breakdowns of the data from the SKI database. Table 1 shows the numbers of failures sorted by pipe size. The first set of data contains 1055 reports where the pipe size was reported in ranges: less than 1 inch, 1 to 4 inches, 4 to 12 inches and greater than 12 inches. Most of the failures were in pipes of size less than 1 inch. The next set of data contains 382 reports where the exact size is unknown, but it could be classified as either less than 1 inch, greater than 1 inch, or a reducer. Lastly, 74 of the reports studied gave no indication of the pipe size. This shows most pipe failures in the reporting period were in pipes of less than 1 in.

Table 1. Number of Piping Failures for Various Pipe Sizes and Pipe Size Categories

\begin{tabular}{|c|c|}
\hline Pipe Size/Category & Number of Failures \\
\hline Actual Pipe Size & 574 \\
\hline$\leq 1$ inch & 252 \\
\hline$>1$ inch \& $\leq 4$ inches & 155 \\
\hline$>4$ inches \& $\leq 12$ inches & 74 \\
\hline$>12$ inches & 1055 \\
\hline Subtotal & 227 \\
\hline Pipe Size Category & 142 \\
\hline “<1” & 13 \\
\hline “>1" & 382 \\
\hline Reducer & 74 \\
\hline Subtotal & 1511 \\
\hline Unknown/Undetermined Size/Category & \\
\hline Total & \\
\hline
\end{tabular}

The next data table from the SKI report, Table 2, shows the number of failures from the report based on the failure type. Failures from leaks were the largest category of failures. This is expected, as the LBB principle from before has described. It is these types of failures being examined in this research. It must be assessed how these pipes failed and the leaks occurred.

Table 2. Number of Piping Failures by Type of Failure

\begin{tabular}{|c|c|}
\hline Failure Type & Number of Failures \\
\hline Leak & 1274 \\
\hline Leak & 54 \\
\hline Crack/Leak & 64 \\
\hline Failed & 13 \\
\hline Rupture & 76 \\
\hline Breakage & 30 \\
\hline Rupture & 1511 \\
\hline Severed & \\
\hline Total & \\
\hline
\end{tabular}

Finally, Table 3 discusses the failure mechanisms described in each report for each type of piping failure. This shows the two biggest known causes of failure as being Fatigue-Vibration (FV) with 364 failures, and Erosion/Corrosion (E/C) with 295 failures. The SKI report notes FV failure is mostly a contributor to pipes 1 in and smaller, but is not visible in any of the presented tables. The SKI report describes a downward trend in failures 
after 1983, contributed to by changes in reporting requirements and increased safety standards, in all areas except ruptures. This is due to $\mathrm{E} / \mathrm{C}$ being the main cause of ruptures. The experiments in this project will seek to recreate these two failure types in tested pipes with machining.

Table 3. Number by Piping Failures for Each Failure Mechanism Category

\begin{tabular}{|c|c|}
\hline Failure Mechanism (Code) & Number of Failures \\
\hline Corrosion/Fatigue (C/F) & 14 \\
\hline Construction Defects/Errors (CD) & 184 \\
\hline Design-Dynamic Load (DDL) & 8 \\
\hline Water Hammer (WH) & 35 \\
\hline Fatigue-Vibration (FV) & 364 \\
\hline Erosion/Corrosion (E/C) & 295 \\
\hline Stress Corrosion / IGSCC SC & 166 \\
\hline Corrosion (COR) & 72 \\
\hline Thermal Fatigue (TF) & 38 \\
\hline Other (OTH) & 43 \\
\hline Unknown Causes (UNK) & 292 \\
\hline Total & 1511 \\
\hline
\end{tabular}

CFEL pipe leakage experiments started with the failures noted above in mind. The piping examined had a nominal diameter of less than 4-inches and focused on FV and E/C failure. Cracks were machined like some of the example papers presented here, as recreating FV and E/C proved too difficult to recreate accurately in the scope of this experiment. A safety advisory from the Canadian National Energy Board [2] indicated FV failure tended to happen where small diameter pipes tie into larger pipes. The failure occurs because these small pipes are not supported well enough, so vibration cause bending stresses at the pipe junction. This situation, as well as $\mathrm{E} / \mathrm{C}$ failure would have taken too much time to recreate in the scope of this experiment. No ruptures were examined for this experiment-leaks through cracks were the focus. Lastly, the specific pipe material was needed.

Most of these small-bore pipes are likely either fire suppression systems, or for water lines for human use (sinks, toilets). The design of the experiment will be based on the pressure ratings of these lines, specifically fire sprinklers. From the National Fire Protection Association's NFPA 13 Standard for the Installation of Sprinkler Systems [3], the minimum pressure rating for fire sprinklers is $7 \mathrm{psi}$, and most fire sprinkler heads are rated to a maximum about 150-175 psi. Fire sprinklers will be chosen for the model as they are ubiquitous not only to every plant, but most plant rooms as well. One aspect of fire sprinkler lines not to be examined is the impurities present in fire lines over time. This is one-way fire sprinkler lines can leak, as water sits in the piping for long periods of time and rusts. The water becomes filled with particulate, which may affect the way water leaks. It would be difficult to recreate this in the available lab space, although it is worth acknowledging that the water in the lab contains a noticeable amount of sawdust, dust, dirt, and other contaminants, due to the open tank. Some of this may be pumped into the test piping, but it would be difficult to quantify how much and what effect it would have.

NFPA specifies the types of piping which may be used in fire sprinkler lines as well. Usable piping types include ferrous piping (welded and seamless), copper tube, CPVC, brass pipe, and stainless-steel pipe. Fittings can be cast iron, malleable iron and steel in addition to the types previously mentioned. The type of pipe chosen for these experiments is schedule 40 galvanized steel. This falls into the category of ferrous piping, and is like the black steel piping often used for fire sprinkler. This was chosen over other materials, as it seems to be the most common material used for sprinkler piping. 
The Idaho National Laboratory published a report in September 2016 [4] concerning the modeling of seismic events in NPPs with advanced probabilistic risk assessment tools. It uses many codes and programs such as EMRALD, SAPHIRE, Mastodon, and Neutrino. The part of highest interest to this research is what they include as the needed enhancements for this demonstration. Most significant for the real pipe leak experiments is the mention of a lack of spray patterns for areas affected. This may be an issue of how the simulation is modeling spray, as the simulation only emits particles in the given direction. Figure 29 shows the figure included in the report with a drawn-in-example of the types of spray patterns they desire. These real pipe spray experiments will endeavor to perform these kinds of simulations in how sprays behave, from the flowrates to the pattern and area affected.

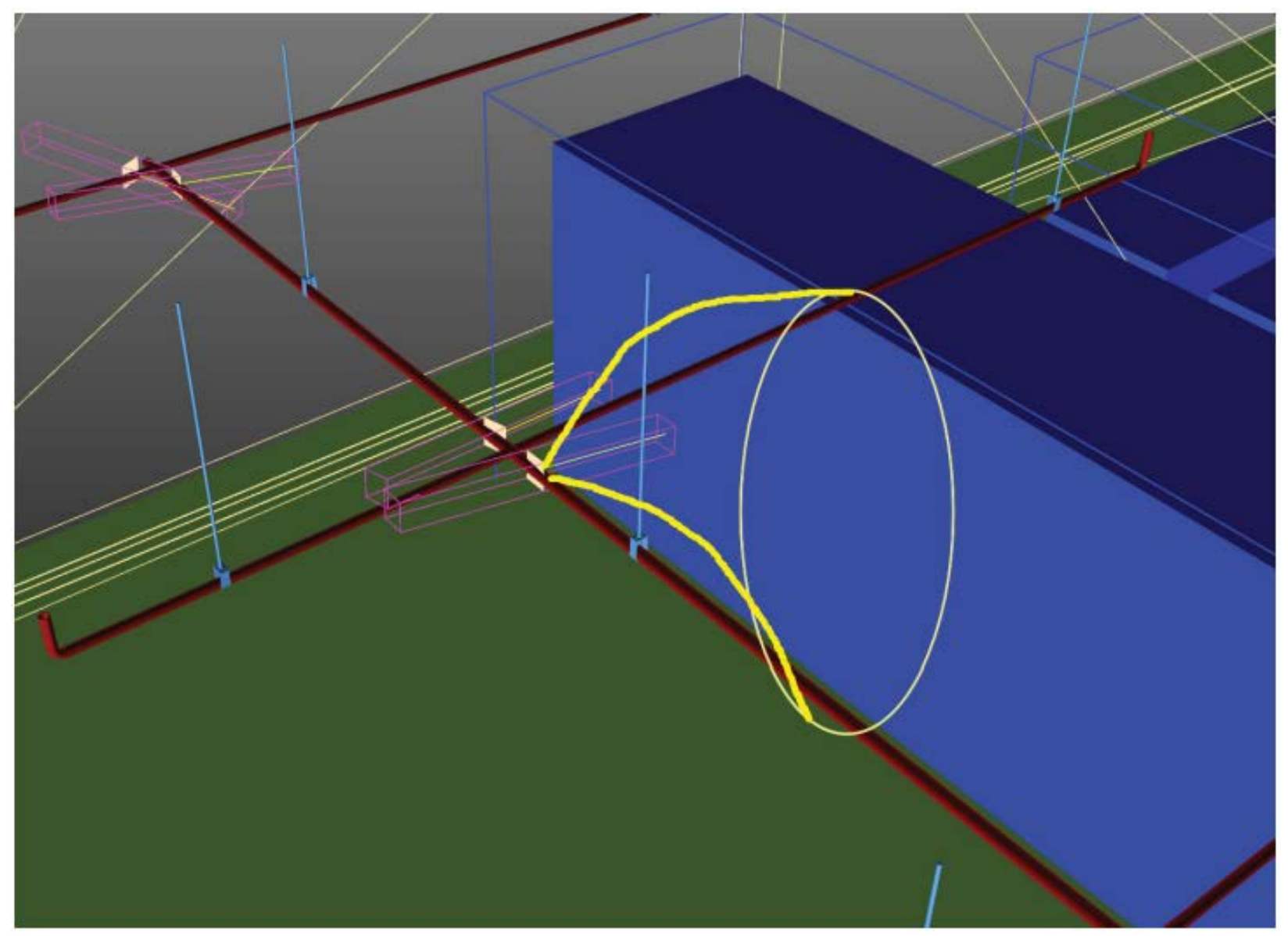

Figure 29. Needed emitter with spray pattern capability.

The goal of the experiment design was to create a piping setup allowing for fast and easy adjustments in the diameter of the failed pipes examined, as well as the failed pipes themselves. The design also needed to be noninvasive to allow for other experiments to take place in the PET. A pump is present in the Water Resource Laboratory from a previous experiment for rising water door failure tests. This pump is a 3-inch high volume dewatering sump pump from Global Pump. The PET was originally designed to accommodate this pump and the 3-inch piping associated with it. The literature review found most pipe leaks were in pipes less than or equal to 1inch, with the next most likely being in pipes between 1 and 4-inches in diameter. Based on this, three pipe diameters were chosen for these tests: 1-inch, 2-inch, and 3-inch sections.

It is useful to know what kinds of flows would be expected from these experiments, so an examination of the piping system was performed for each test section individually to determine the operating point for each line. 
These calculations were made with a few assumptions. First, it was assumed any tee joints the water flowed through with one direction ending in a closed valve were modeled as regular elbows. Next, for reducing tees, with flow only going one direction, the same assumption as before was made, but also including a sudden reduction in the line. The next assumption is for the starting point and the end. It is assumed the starting point is at the surface of the water in the tank, and the outlet back into the tank is the exit. This difference in height was measured to be 14.5 inches at the time of the experiments. The result of this can be seen in Figure 30. Three curves were developed, as there are three different operating conditions. The smaller the pipe diameter, the higher the head losses, and the lower the operating flow. The larger the pipe diameter, the lower the major head losses from friction, so a higher flow with a lower pressure is expected.

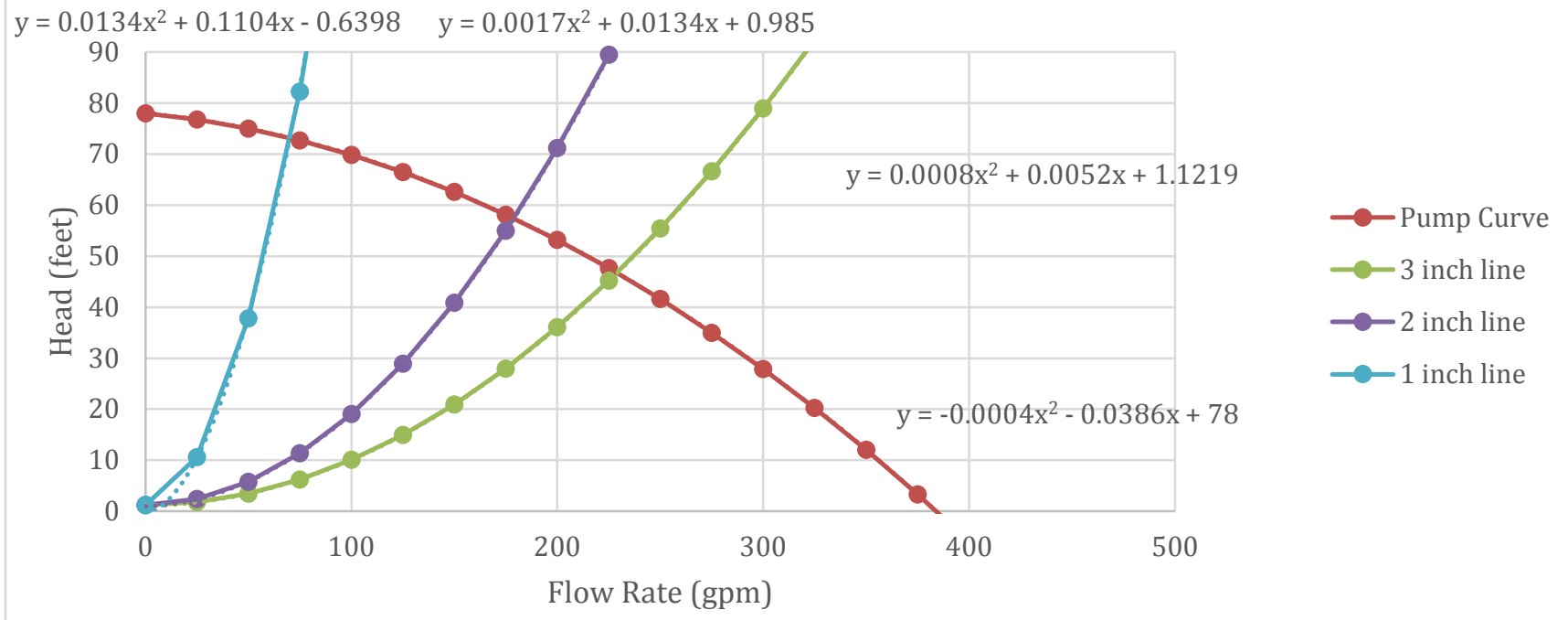

Figure 30. Pump curve operating point calculations.

Figure 31 shows the completed test section inside the PET. Three test sections, of three different pipe diameters (1-inch, 2-inch, and 3-inch), are built in and are intended to run independently. Each test section has a u-bend of galvanized steel piping. The galvanized pipe is where the failure will be produced, because of its similarity to fire sprinkler piping. Fire sprinkler piping is generally black steel piping as it is more fire resistant. Fire sprinkler lines can range in size from schedule 10 to schedule 40 [3]. Galvanized pipe was chosen over black steel as the parts in the size required were more readily available. Failure will be induced with milling equipment to ensure a slot of consistent and known size can be created. The u-bend setup allows for several positions of cracks in piping to be tested, as failure can be tested in horizontal and vertical sections of piping, as well in elbows. On each piece of piping, different orientations of cracks can be tested as well - for instance, on pipe runs, two simple slots can be created, circumferentially or longitudinally. For simplicity in the rest of this thesis, parts of the piping will be referred to as either upstream or downstream of the test sections. 


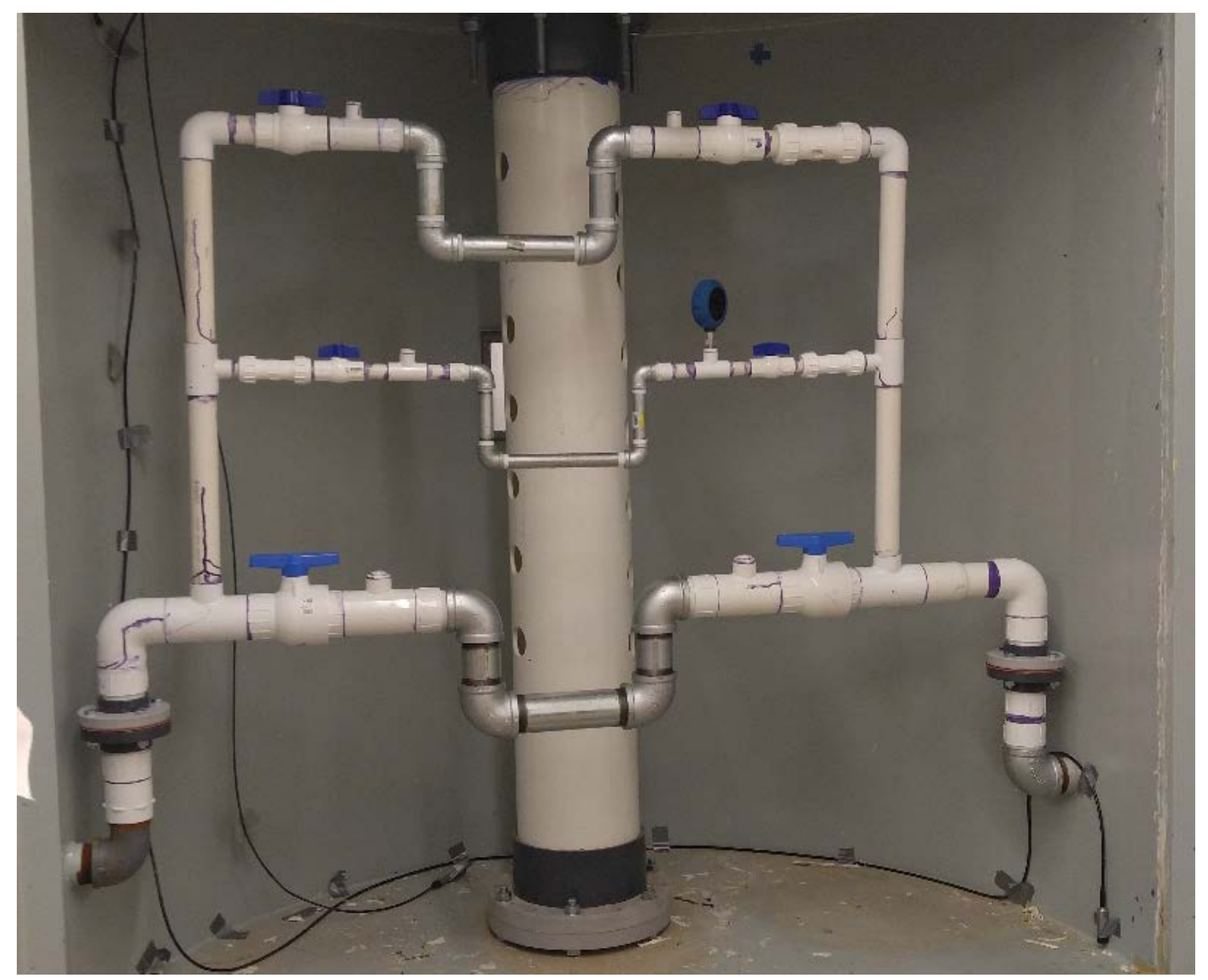

Figure 31. Pipe rupture spray pattern configuration.

In addition to the u-bend section of galvanized steel, each test section included two valves on each side to isolate each test section when they are not being tested. Inside of the valves are two tees reducing to $1 / 4$ inch to allow for the installation of pressure gauges. The idea being if the pressure on each side of the piping can be known, with and without any failures, then the pressure loss through the crack will be known as well. The location of one of these gauges can be seen in the middle test section in Figure 31 to the right of the steel pipe. The 1-inch (middle section) and the 2-inch (top section) piping also include compression couplers in their design. This is to allow easy removal and replacement of test section components once experiments begin.

Figure 32 shows a P\&ID diagram for the setup. The different pipe sizes used are denoted by the different line thicknesses, and the steel test section has been denoted with red lines. This diagram makes it appears there are six pressure gauges, two for each leg, however this is just to demonstrate the places in the diagram where the pressure gauges are used in each line. The valves are numbered as they will be referred to in following sections. Each line has four different valves associated with it. Valves 1 and 4 are the 3-inch brass valves on the outside of the PET, and are the same for all test setups. Valves 2 and 3 are line dependent, but refer to valves in the same position relative to each line's test section. Valves will be referred to with these numbers from here, or as upstream/downstream and PVC/brass. 


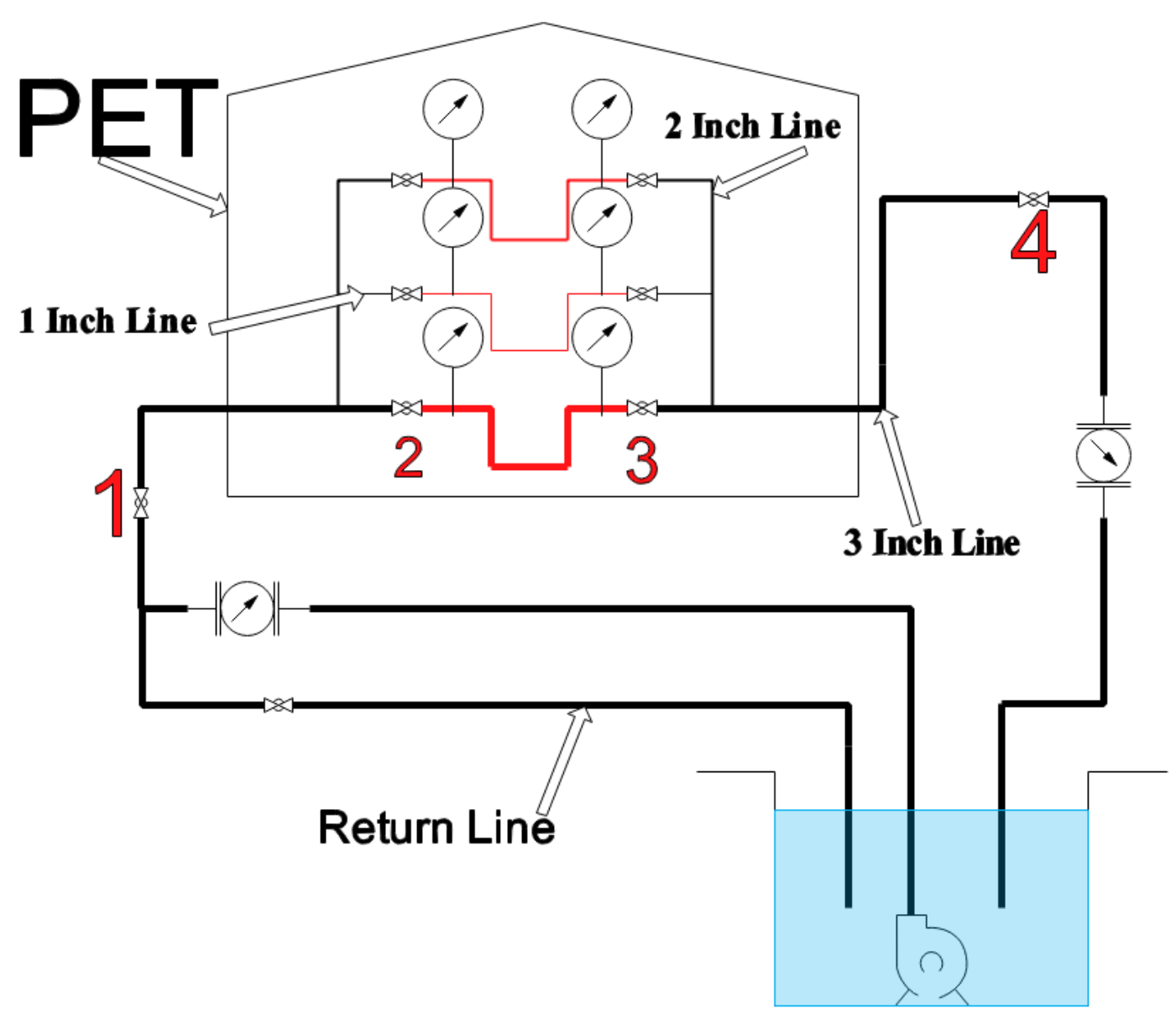

Figure 32. P\&ID diagram of pipe leak setup.

The experimental procedure is simple. After installing the failed pipes for all three diameters, set all the valves to full open for one line. Close the two PVC valves on each of the other lines to isolate them. Set up a camera to record the leak. Turn the pump on and record the flow data, the pressure data, and the videos, as well as taking any necessary pictures. After recording for four minutes, partially close valve 1 , then record for four more minutes. Continue downstream through the valves, partially closing them, then recording for four minutes. After all, four valves are partially closed and recorded, open valves 1, 2, and 3, then working from downstream to upstream repeat the recordings, closing valve 3 then 2 . Valve 1 was not partially closed from downstream to upstream because it would be repeating data already collected upstream to downstream.

Three leak types were tested in this experiment. All the cracks were milled using a thin blade in a milling machine. The first failure type tested is a longitudinal crack. The tested section is the middle, galvanized nipple seen in Figure 31 for each diameter. The crack was made to be 2 inches externally. As the disk used to cut it was circular, the inner hole is not the same 2 inches and is slightly smaller. The size of the hole was measured with calipers and can be seen in Table 4. Pictures of the three nipples can be seen in Figure 33. 
Table 4. Longitudinal Slit Measurements

\begin{tabular}{|c|c|c|c|}
\hline & 3-inch & 2-inch & 1-inch \\
\hline Length (inches) & 1.970 & 1.978 & 1.990 \\
\hline Width (inches) & 0.039 & 0.049 & 0.042 \\
\hline Area $\left(\right.$ in $^{2}$ ) & 0.077 & 0.097 & 0.084 \\
\hline
\end{tabular}

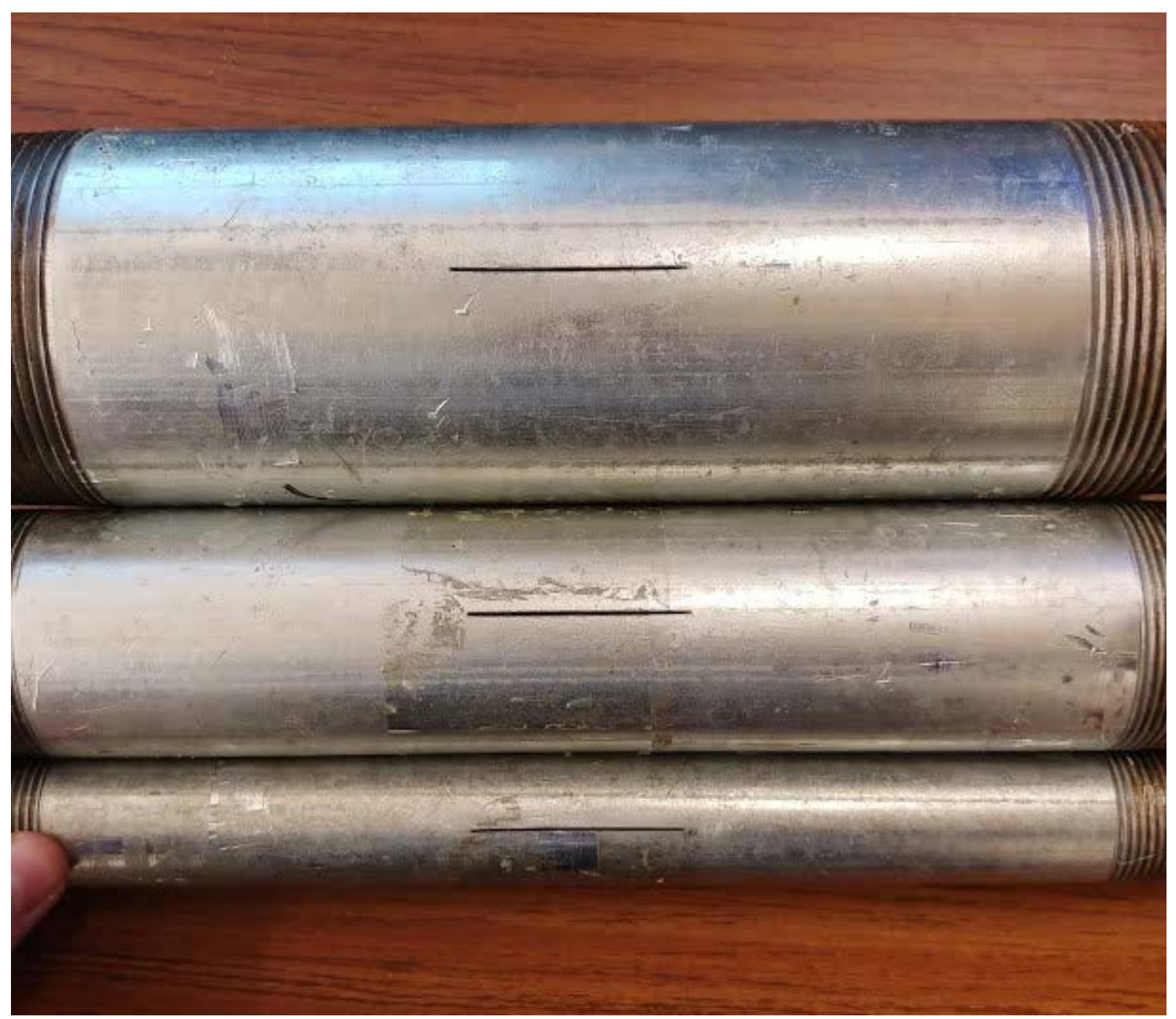

Figure 33. Longitudinally slotted galvanized nipples.

The next crack type is a circumferential crack on the same straight nipple section (referred as circumferential from here on), on new pipe. As the pipes have different diameters, the cracks were milled to be about a $90^{\circ}$ arc. It was wished to have the cracks in the middle like the longitudinal cracks, but it ended up not being feasible for the machinist to mill it in the middle. The cracks ended up being about 3 inches from one of the ends. Table 5 shows the arc length for each crack, the width of the crack, and the depth. Figure 34 shows the three nipples side by side. 
Table 5. Circumferential Slit Measurements

\begin{tabular}{|c|c|c|c|}
\hline & 3-inch & 2-inch & 1-inch \\
\hline Length (inches) & 2.707 & 1.835 & 1.057 \\
\hline Width (inches) & 0.039 & 0.043 & 0.037 \\
\hline Length from End & 3.014 & 2.939 & 3.021 \\
\hline Area $\left(\right.$ in $\left.^{2}\right)$ & 0.083 & 0.062 & 0.031 \\
\hline
\end{tabular}

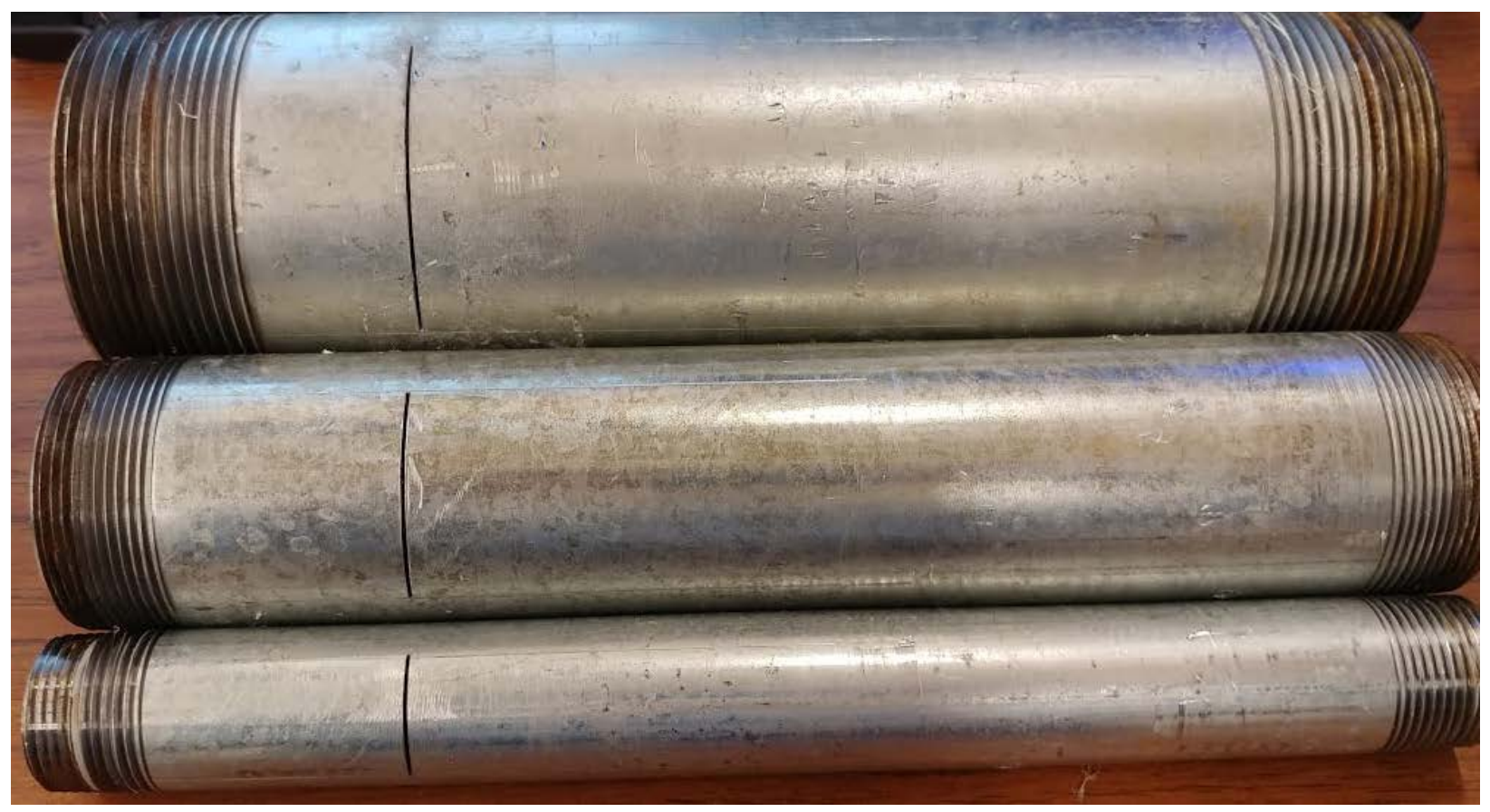

Figure 34. Circumferential slotted galvanized nipples.

The last type of crack tested was on the outside of a threaded elbow. The elbow position of the elbows can be seen in Figure 35. This position was chosen as it allowed the water to have a straight run before the leak, as well as being positioned vertically without otherwise changing the piping. Other positions would have required repositioning of the test section to have the slit vertical. The same idea was taken for the elbows as was the circumferential cut pipes, the elbows were cut circumferentially to a $90^{\circ}$ arc, centered on the back of the elbow. A picture of the three elbows can be seen in Figure 35 and Table 6 shows the size of the cracks and the arc length of the cracks.

Table 6. Elbow Slit Measurements

\begin{tabular}{|c|c|c|c|}
\hline & 3-inch & 2-inch & 1-inch \\
\hline Length (inches) & 3.131 & 2.162 & 1.356 \\
\hline Width (inches) & 0.045 & 0.041 & 0.038 \\
\hline Area $\left(\right.$ in $^{2}$ ) & 0.111 & 0.070 & 0.040 \\
\hline
\end{tabular}




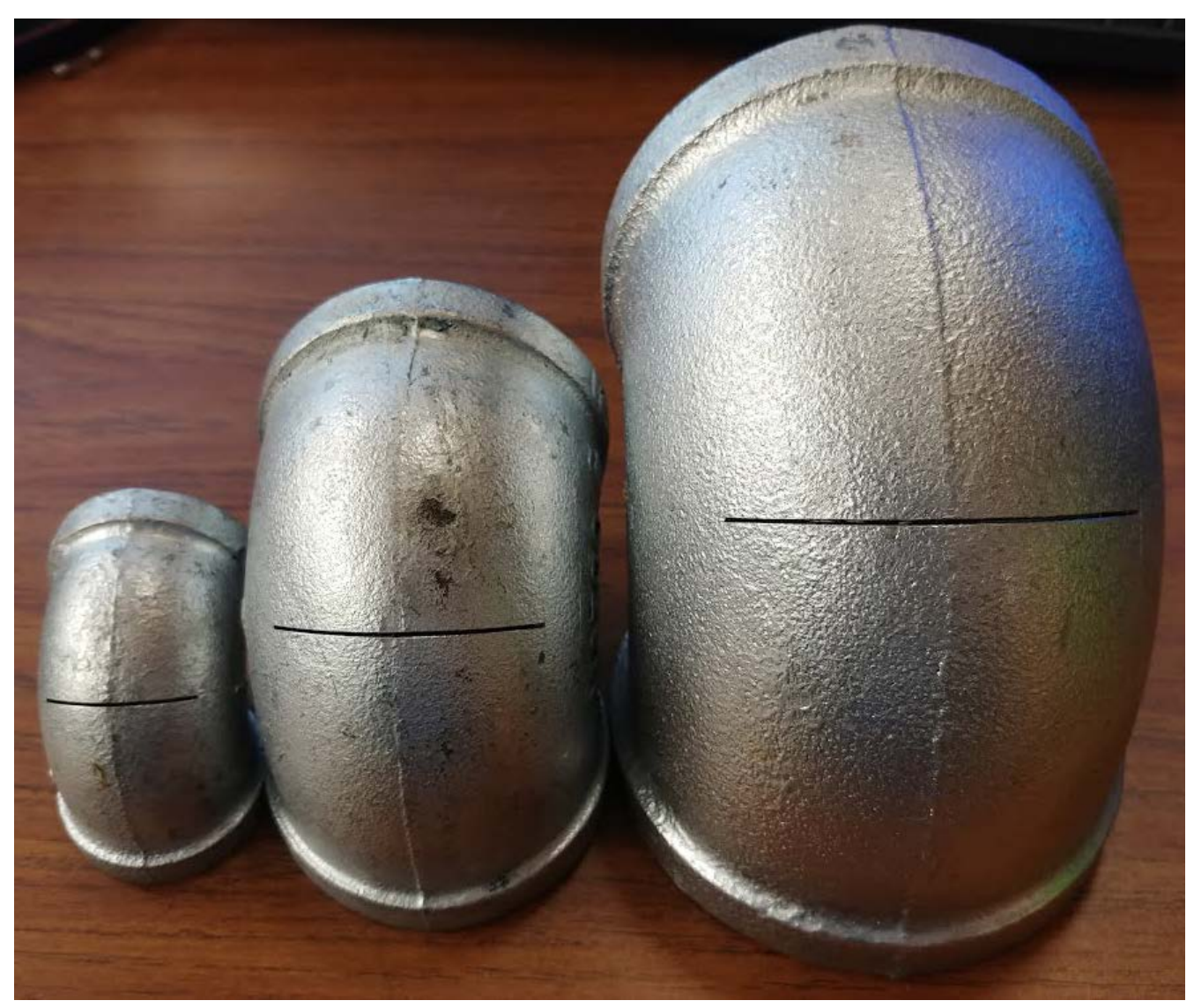

Figure 35. Galvanized slotted elbows.

There are several results of the leak tests this section will seek to present, some quantitative, some qualitative. The qualitative results are difficult to define, but will seek to describe the spray and pattern in a categorical way. Each different crack style will be described and shown with typical traits of each crack. Different categories will be presented then with different examples from the three different crack styles. The demonstrations will mostly be done visually through videos and photos, and with some description. Each four-minute test had at least one picture and one video taken of the spray. These will be used as the evidence for these categories. As videos cannot be used here, stills will be presented as applicable.

The first crack type examined was longitudinal. As discussed above, the cracks made in the pipes were approximately 2 inches long placed in the center of the pipes. This is the type of leak expected to be most like an $\mathrm{E} / \mathrm{C}$ failure. This is the most directed type of the three sprays, coming out in a stream from the crack. There seems to be a velocity or pressure dependence on the angle of the stream as it exits the pipe. This will be explored further below. The spray often oscillates, which can be seen in Figure 36. Figure 36 (progressing left to right, top to bottom) shows the oscillation of a spray with no valves closed. The frames were taken over the course of 14 frames in the video recorded at 120 frames per second (fps). This makes the period of oscillation for this configuration is about 0.12 seconds. The oscillations visible here are likely due to variations in the pump during operation. Similar oscillations may not be present in systems where the water supply is more constant. 


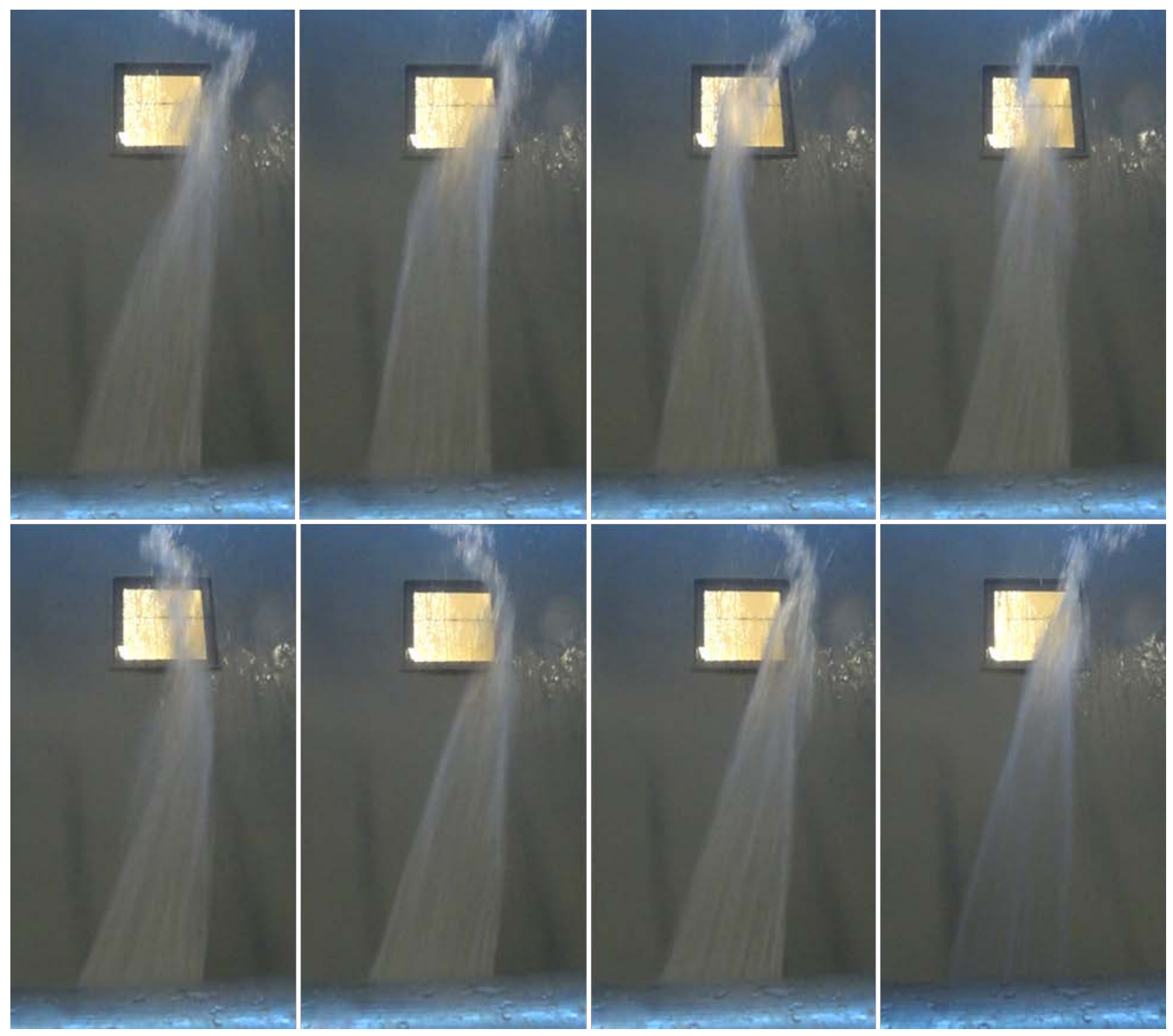

Figure 36. Oscillation in longitudinal slit.

Figure 37 shows a wider view of the same 3-inch-no-valves-closed experiment. The water sprayed toward the back of the tank where it hits above the window (oval area) then splashed upward towards the ceiling, where some spray can be seen. The upwards direction seemed to be directly caused by the angle of the pipe in the threads, it appears the water came out normally through the pipe, and not at some upward angle. This type of failure is unique from the other two, as the cracks in all three pipes are of comparable size. 


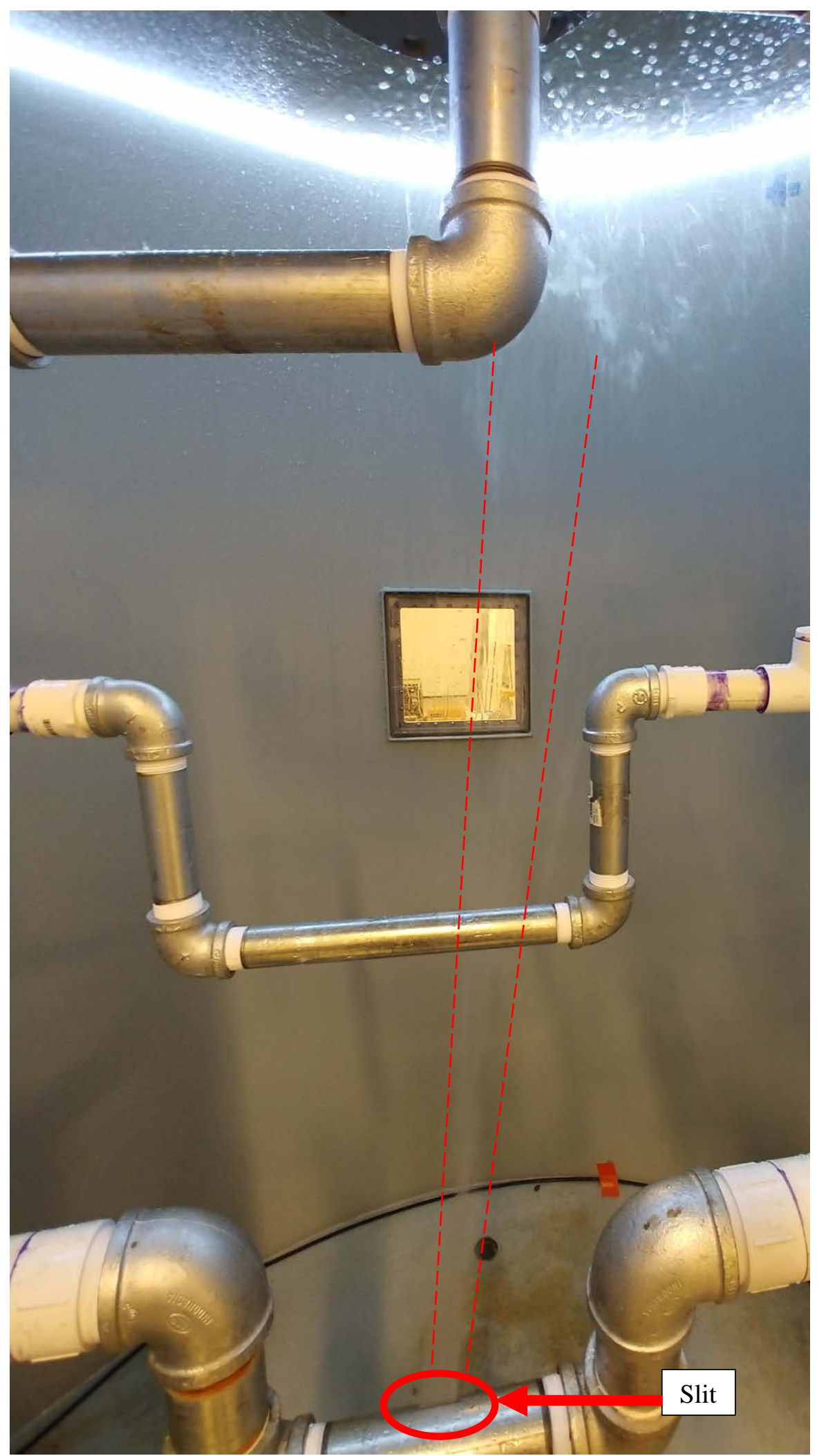

Figure 37. Larger view of longitudinal spray with no valves.

The second crack type is circumferential. This type of crack is most likely related to FV failure, due to the bending stresses created by vibration. This type of crack results in a fan-like spray, as seen in Figure 38. Figure 
39 shows the side view of the same spray. This spray can reach to top of the PET, even though it is not as directed as the longitudinal spray. Circumferential leaks create more mist than the longitudinal slits as well. This means there are smaller water droplets in the air, making it feel far more humid than the longitudinal. This may not be important for components directly in the spray failing, but may cause components to fail indirectly.

The biggest difference between the pipe diameters for this failure type is the difference in the failure area. As described before, the circumferential cracks were created to be as 90-degree arcs around the pipe, so the leakage area was lower for each pipe. Figure 40 and Figure 41 show the two-inch, and the one-inch circumferential cracks, respectively. A prominent feature here is the two-inch circumferential cut has burrs from the machining still inside of the crack. These burrs made the two-inch spray have several small streams, rather than appearing fan like, compared to the three-inch and the one-inch. Lastly, though the one-inch, and the three-inch appear similar, there is notably less flow through the one-inch crack, as expected. Note the clarity of the piping behind the spray is much sharper with the one-inch. Generally, for this crack type, as the pressure increased, and the crack area decreased, the finer the resulting mist, and a lower the distance the mist could travel.

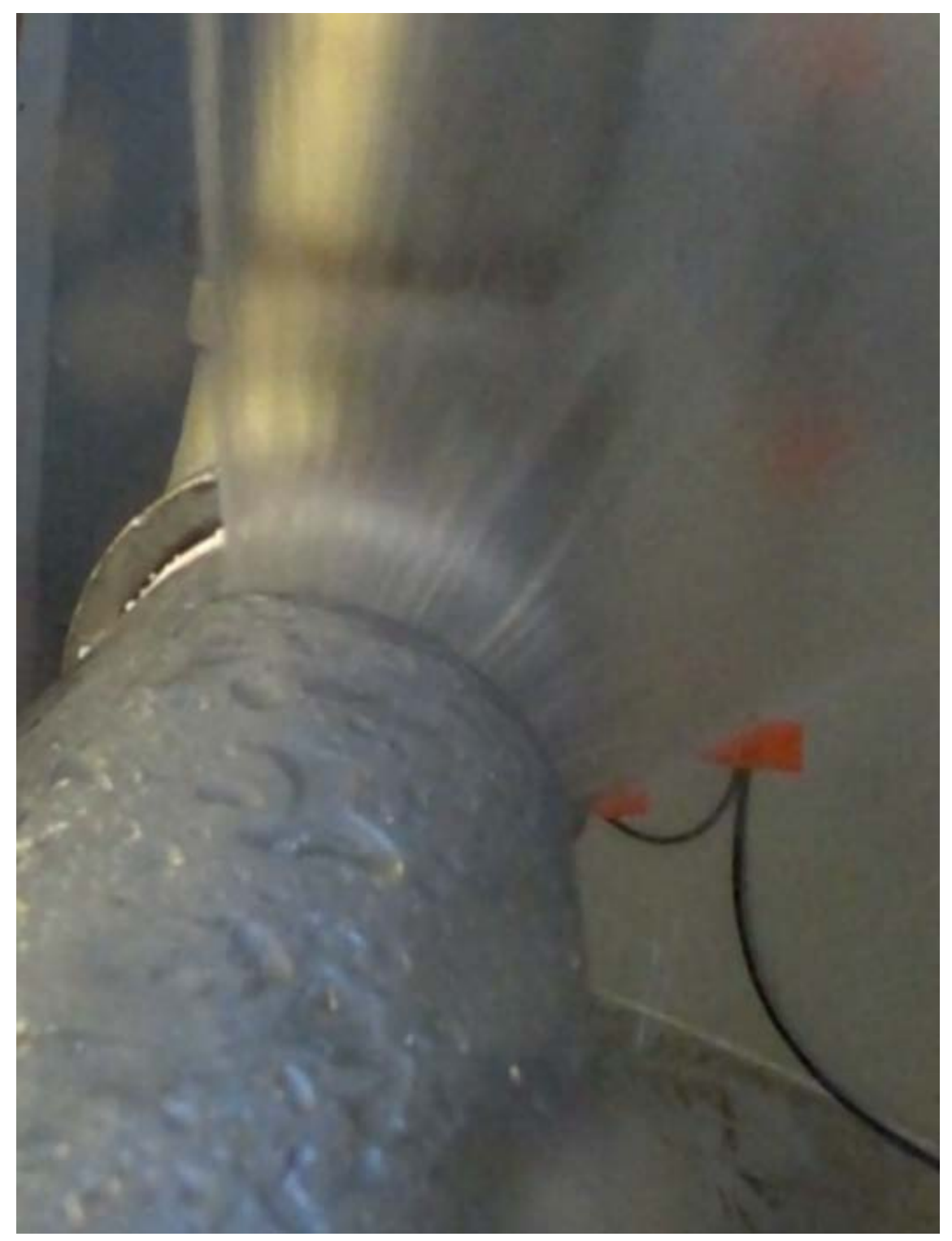

Figure 38. Fan spray from a circumferential crack, 3-inch pipe. 


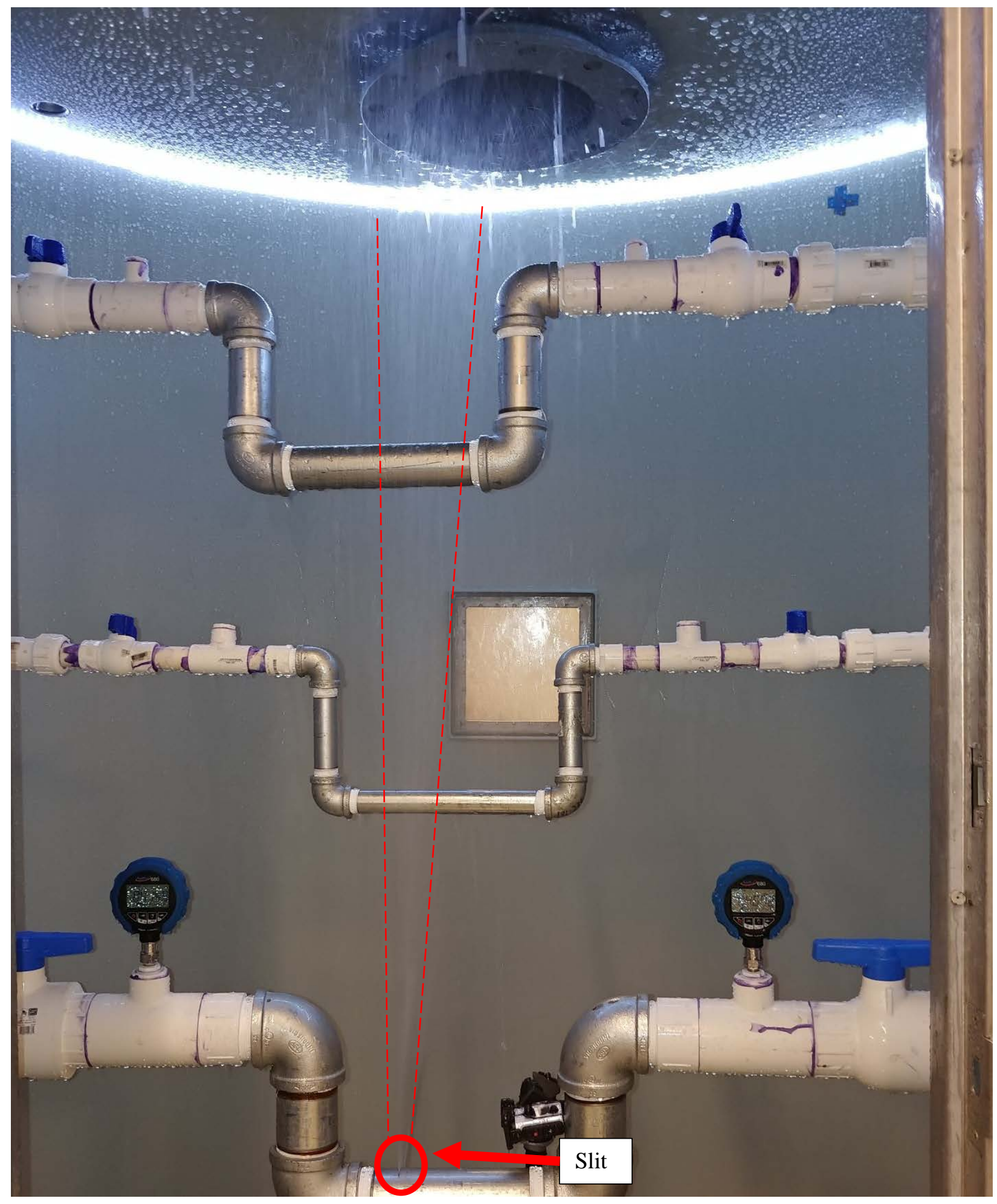

Figure 39. Elevation view of the circumferential spray. 


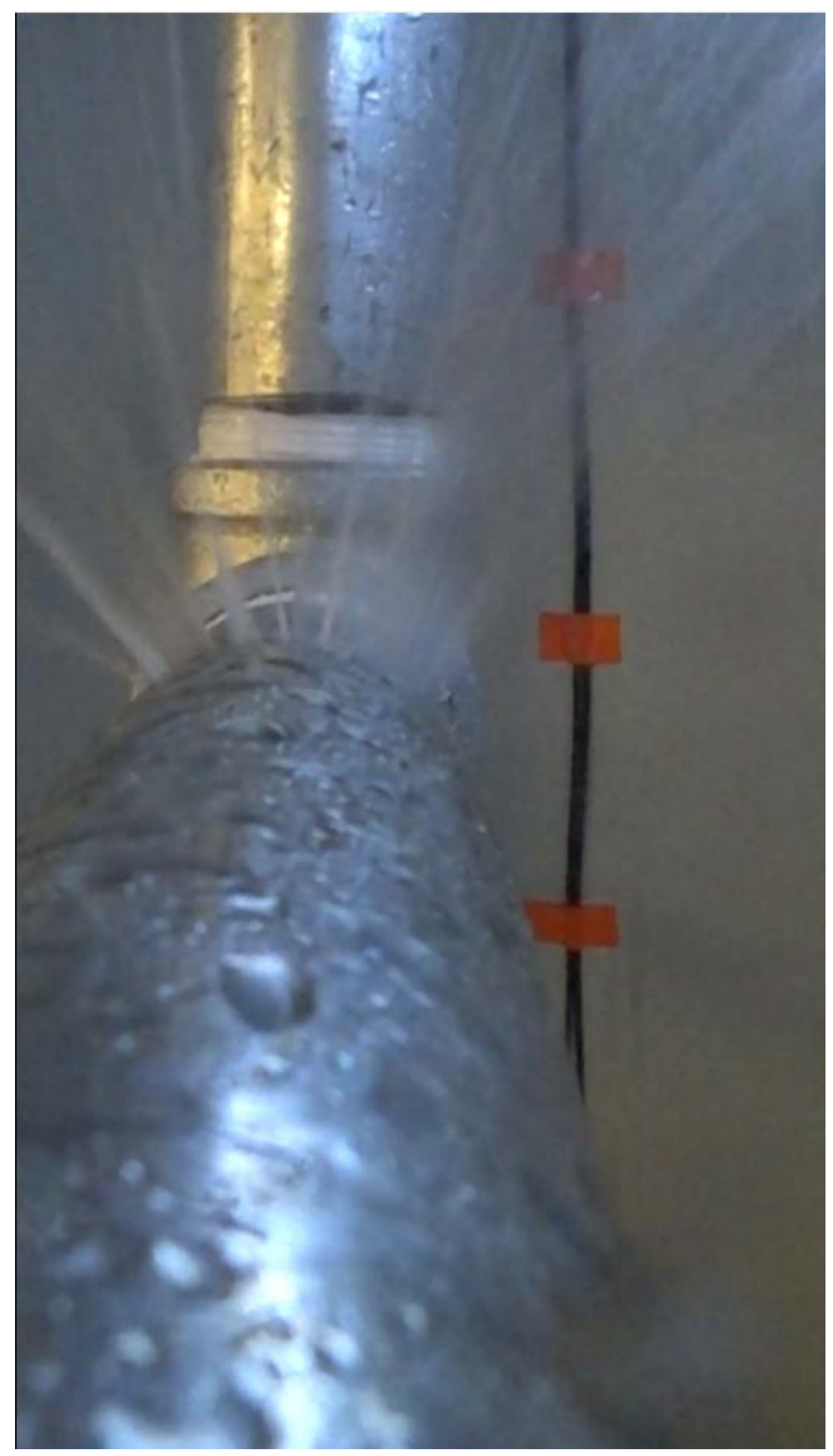

Figure 40. Fan spray from a circumferential crack, 2-inch pipe. 


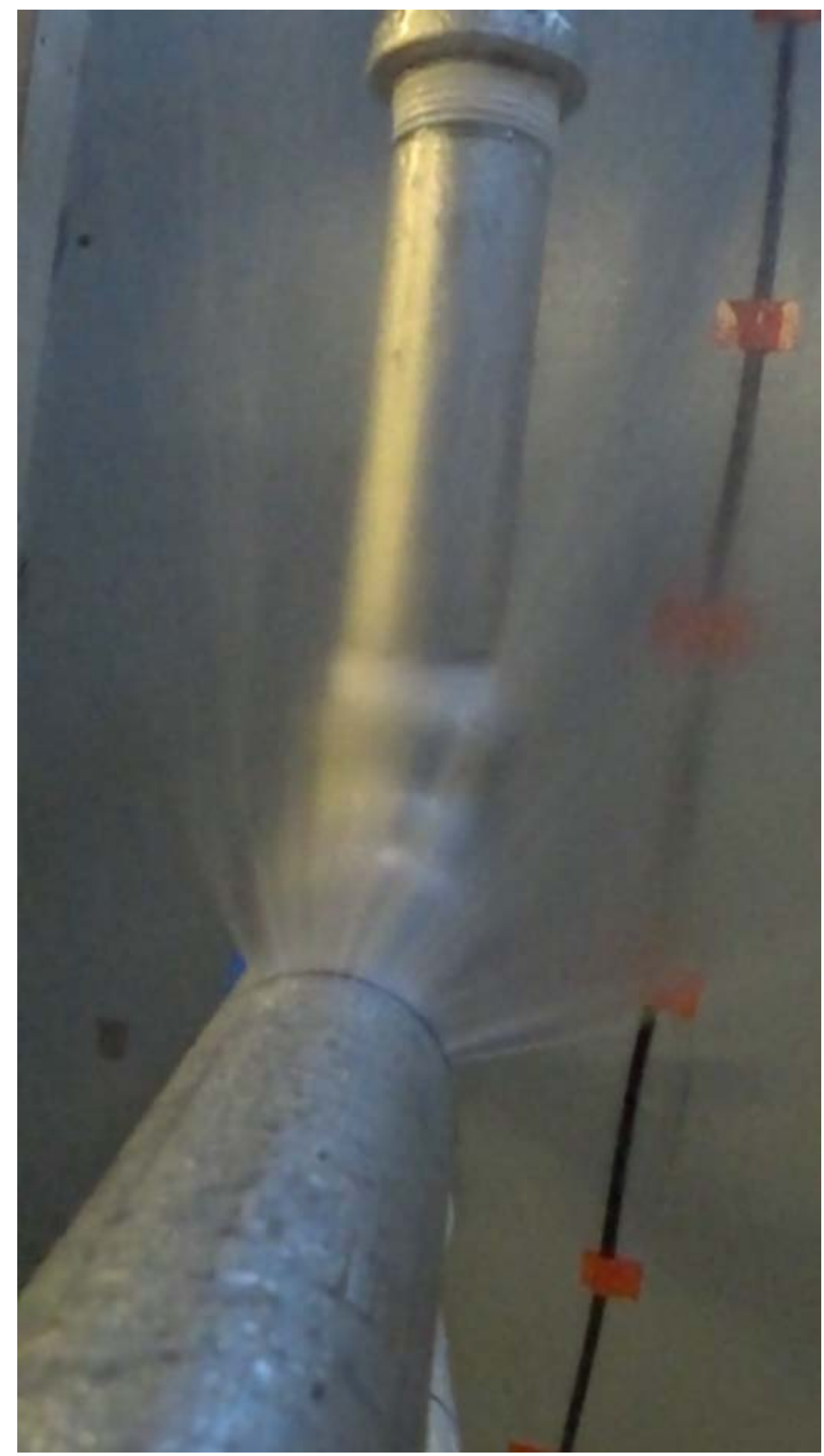

Figure 41 Fan spray from a circumferential crack, 1-inch pipe.

The last type of spray tested was leaks from elbows. Leaks from elbows, especially when they are somewhat circumferential, could also be described as FV failure if the piping had been secured poorly. Figure 42 
shows a side view of an elbow leaking, and Figure 43 shows the view from the video camera, which was placed above the elbow looking down. This type of failure was a combination of the past two, the longitudinal and the circumferential. It is like the circumferential due to the fan-like shape of the spray, as seen in Figure 43. The similarity is not very much of a surprise, as the slit is similarly somewhat circumferential. The elbow spray is like the longitudinal spray in the directed manner of the spray, as can be seen in Figure 42. The elbow leaks tended to have higher leaks compared to the other leak types with similar pipe flows. The higher leak rates are likely due to the position of the elbow, and the changes in momentum occurring. The comparison of the leak rates between the types is discussed more in depth in the next section. The elbow leaks did not appear to show as much of an oscillatory effect from the leaks, or if they did, they are not visible in the videos.

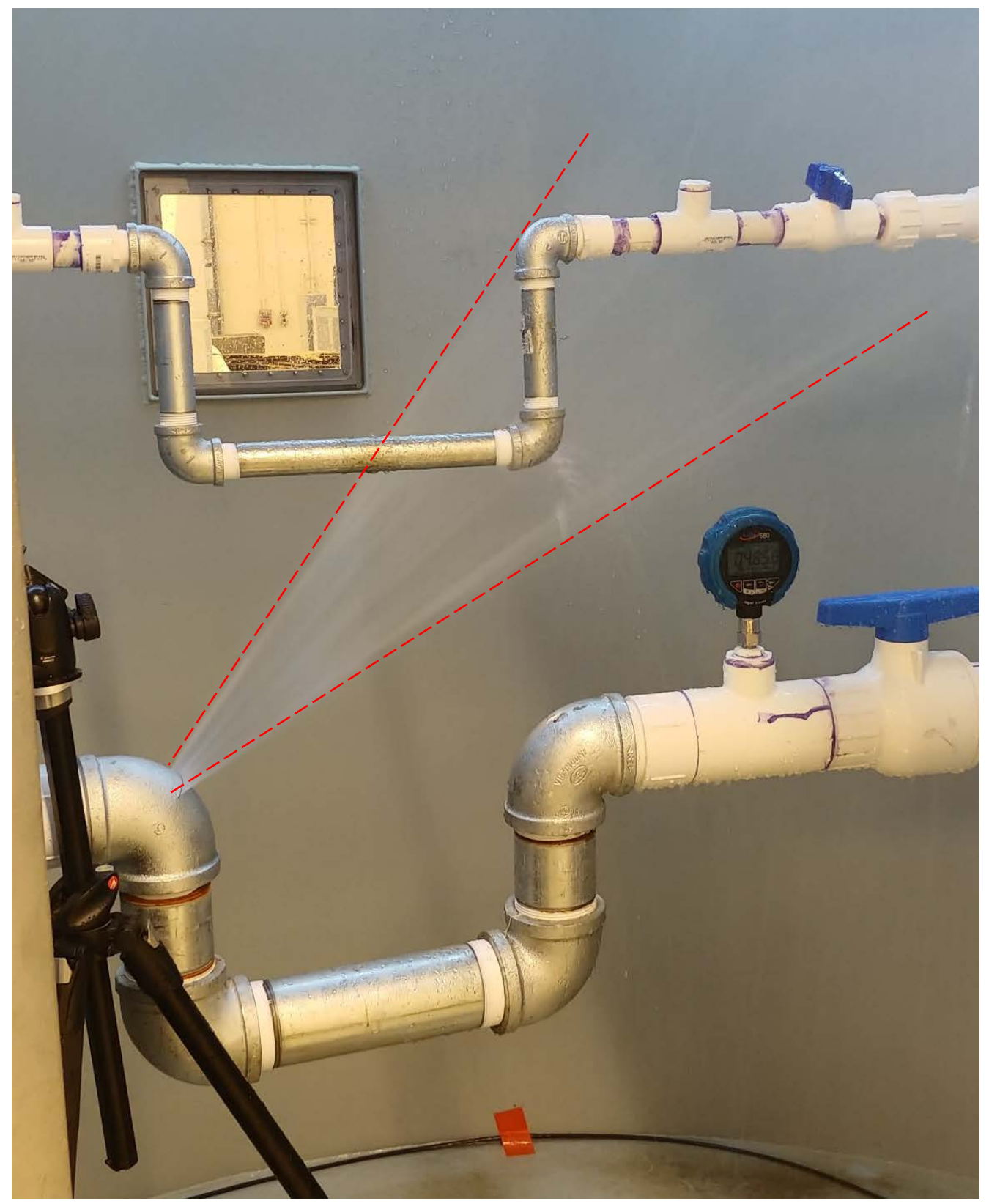

Figure 42. Spray from elbow, side view. 


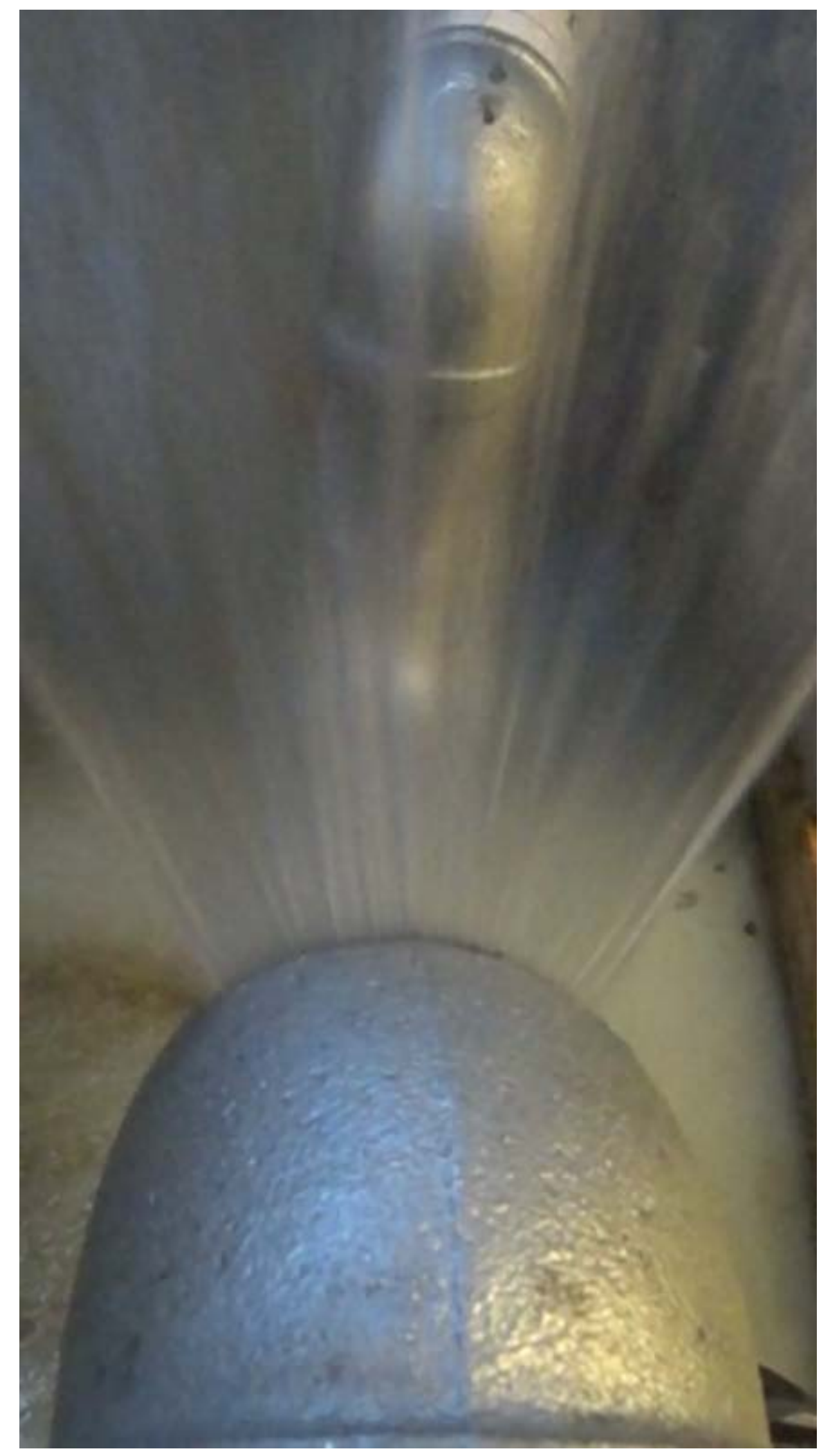

Figure 43. Spray from elbow, straight on view. 
Throughout the course of these experiments three types of sprays were identified. To categorize them, valve configurations were selected since they help identify flow and pressure groupings. The three types are:

1. No valve closed leak

2. Upstream valves closed

3. Downstream valves closed

The Type 1 configuration leaks generally had moderately high leak rates, pressures, and leak velocities. There were no valves creating large drops in pressure and flow. The previously presented pictures are all of Type 1.

Table 7. Type 1 Leak Data

\begin{tabular}{|c|c|c|c|}
\hline & $\begin{array}{c}\text { Leak rate } \\
\text { (gpm) }\end{array}$ & $\begin{array}{c}\text { Pressure at } \\
\text { Leak (psi) }\end{array}$ & $\begin{array}{c}\text { Velocity } \\
\text { (ft/s) }\end{array}$ \\
\hline Longitudinal & & & \\
\hline 3-inch & 4.81 & 6.63 & 20.04 \\
\hline 2-inch & 4.87 & 8.31 & 16.12 \\
\hline 1-inch & 5.40 & 9.94 & 20.63 \\
\hline Circumferential & & & \\
\hline 3-inch & 5.58 & 2.07 & 21.58 \\
\hline 2-inch & 2.89 & 3.35 & 10.19 \\
\hline 1-inch & 1.46 & 3.81 & 8.54 \\
\hline Elbow & & & \\
\hline 3-inch & 6.82 & 7.05 & 19.76 \\
\hline 2-inch & 4.54 & 2.60 & 13.16 \\
\hline 1-inch & 3.22 & 1.65 & 9.35 \\
\hline
\end{tabular}

Departing from the Type 1 leaks, the Type 2 happens when valves upstream of the leak are partially closed. More generally, if there are spots of large resistance upstream of the leak. Type 2 leaks have the lowest flow rates and the lowest velocities from the leaks compared to Type 1 and 3. Figure 44 shows a close view of a Type 2 leak from a longitudinal leak, and Figure 45 shows a wide angle. The water from the leak is barely reaching the back wall of the PET before hitting the ground. The misting from this type of leak is minimal, as can be seen in Figure 46 and Figure 47. These two figures show type leaks from an elbow and a circumferential leak respectively. Both leak types were characterized by the mist they created. At these lower flows and velocities though, very little mist is created. The distance away from this leak type at which components would be expected to be damaged is low, and would likely be only the components directly below the failed piping.

The data in Table 8, other than demonstrating Type 2 leak, also shows another comparison between the three crack types. The data in the table is from the tests with both upstream valves half closed. At low flows, the leakage from the elbow is still the greatest, even in the 1 and 2-inch piping, where the opening is smaller than the longitudinal slit. Conversely, the longitudinal crack leak parameters tend to be lower than the other two crack types. 
Table 8. Type 2 Leak Data Example

\begin{tabular}{|c|c|c|c|c|c|c|}
\hline & $\begin{array}{c}\begin{array}{c}\text { Leak rate } \\
(\mathrm{gpm})\end{array} \\
\end{array}$ & $\begin{array}{l}\text { Error } \\
\text { (gpm) }\end{array}$ & $\begin{array}{c}\text { Pressure at } \\
\text { Leak (psi) } \\
\end{array}$ & Error (psi) & Velocity (ft/s) & Error (psi) \\
\hline \multicolumn{7}{|c|}{ Longitudinal } \\
\hline 3-inch & 1.58 & 0.74 & 0.76 & 0.076 & 6.57 & 0.87 \\
\hline 2-inch & 4.26 & 0.59 & 10.14 & 0.076 & 17.77 & 0.91 \\
\hline 1-inch & 4.55 & 0.60 & 12.26 & 0.08 & 18.97 & 0.92 \\
\hline \multicolumn{7}{|c|}{ Circumferential } \\
\hline 3-inch & 2.21 & 1.11 & 1.87 & 0.076 & 8.54 & 1.15 \\
\hline 2-inch & 4.14 & 0.80 & 7.48 & 0.076 & 16.03 & 0.96 \\
\hline 1-inch & 6.17 & 0.75 & 15.07 & 0.076 & 23.87 & 1.09 \\
\hline \multicolumn{7}{|l|}{ Elbow } \\
\hline 3-inch & 3.22 & 0.77 & 1.65 & 0.076 & 9.35 & 0.66 \\
\hline 2-inch & 6.07 & 0.74 & 8.88 & 0.076 & 17.60 & 0.75 \\
\hline 1-inch & 7.35 & 0.59 & 13.13 & 0.076 & 21.30 & 0.76 \\
\hline
\end{tabular}

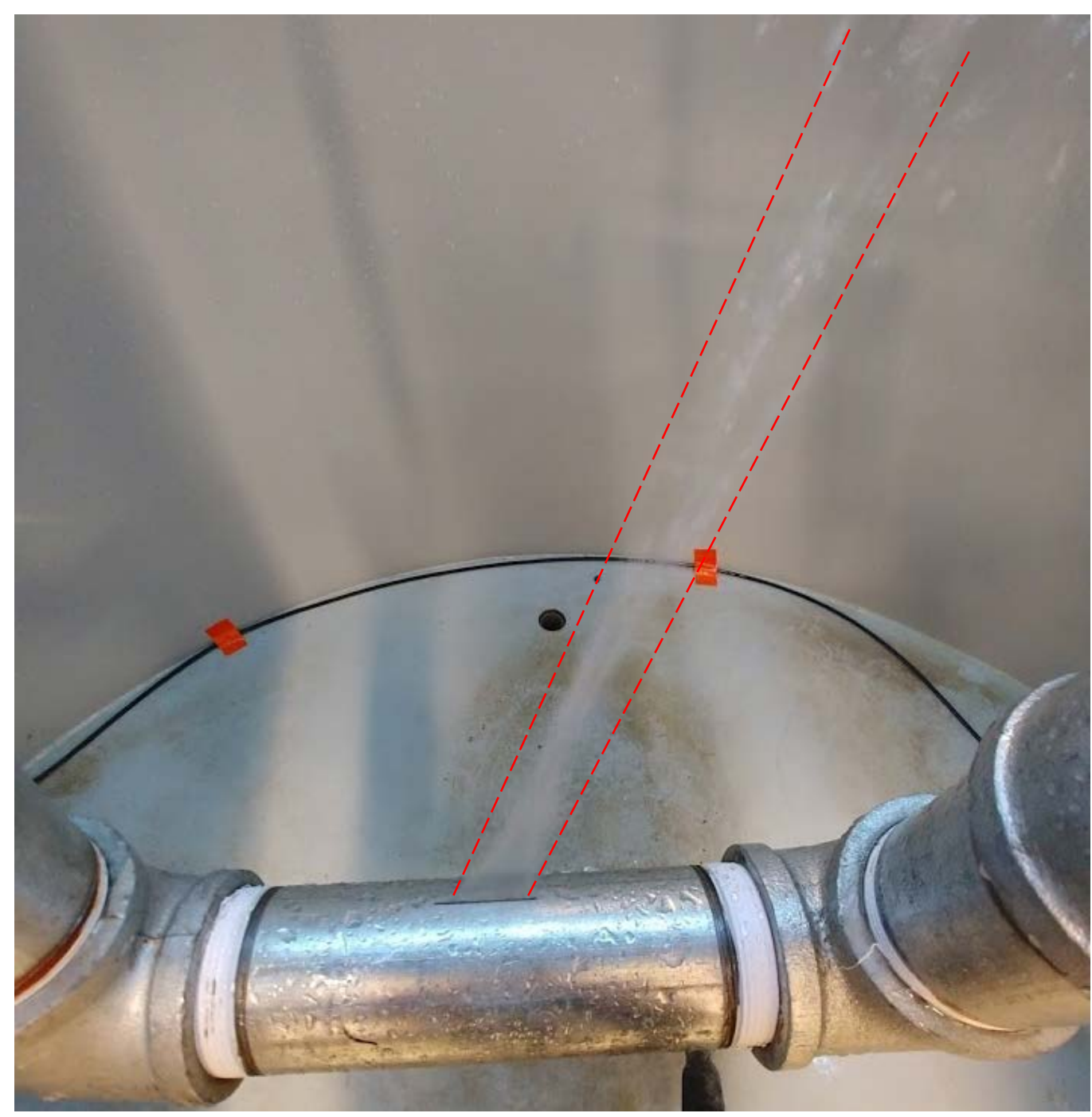

Figure 44. Type 2 leak from a longitudinal crack, close view. 


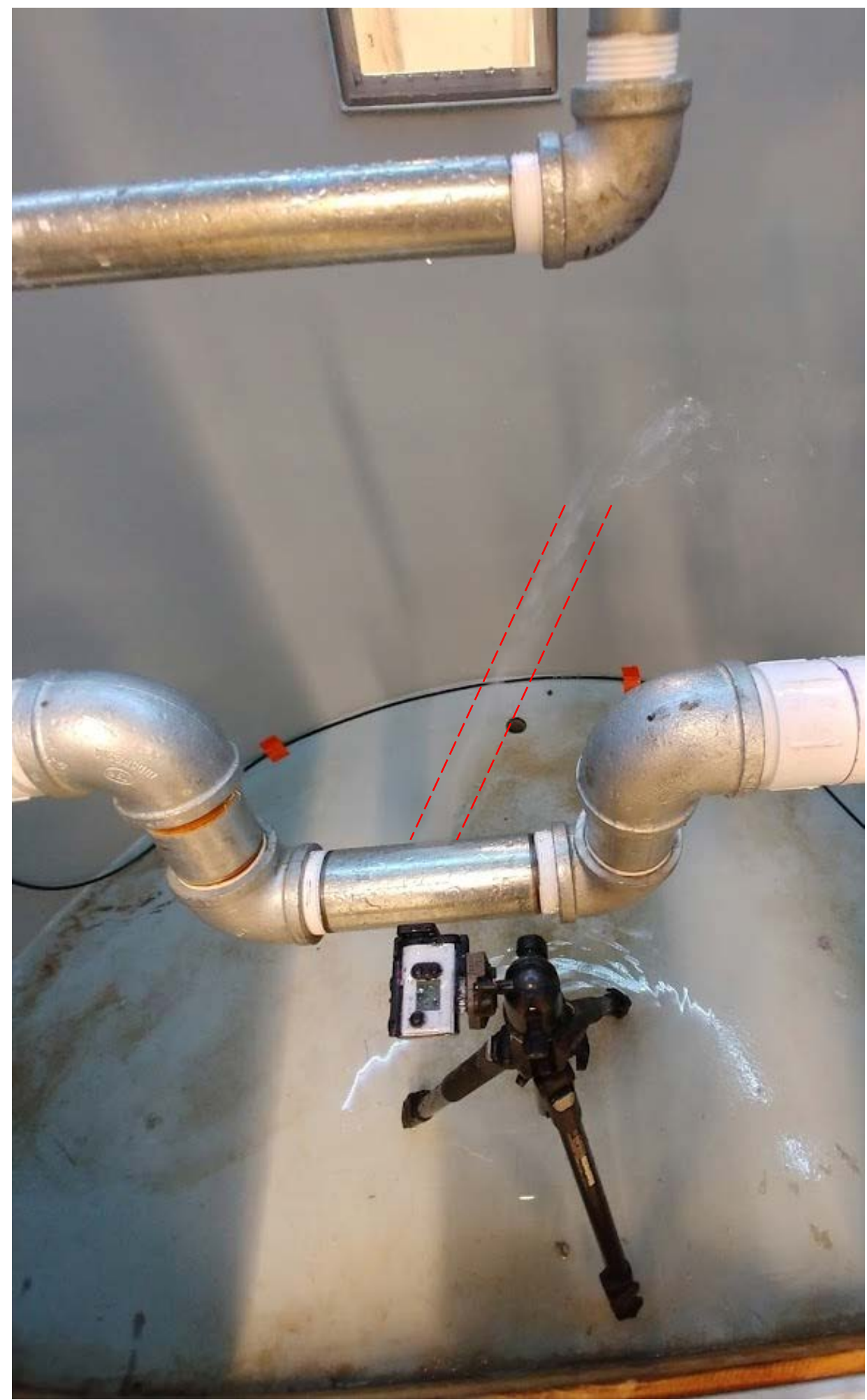

Figure 45. Type 2 leak from a longitudinal crack, wide view. 


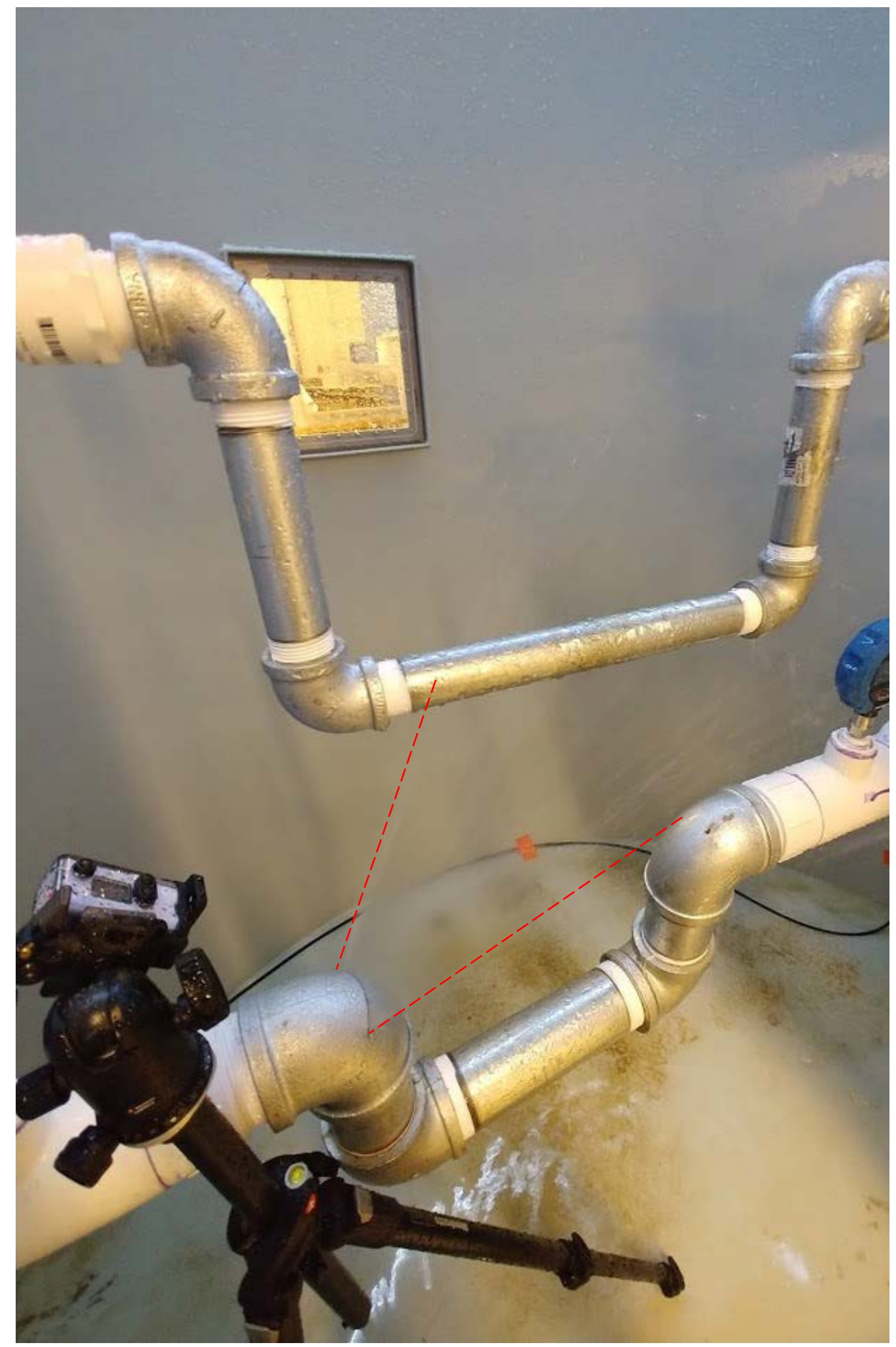

Figure 46. Type 2 leak from an elbow leak. 


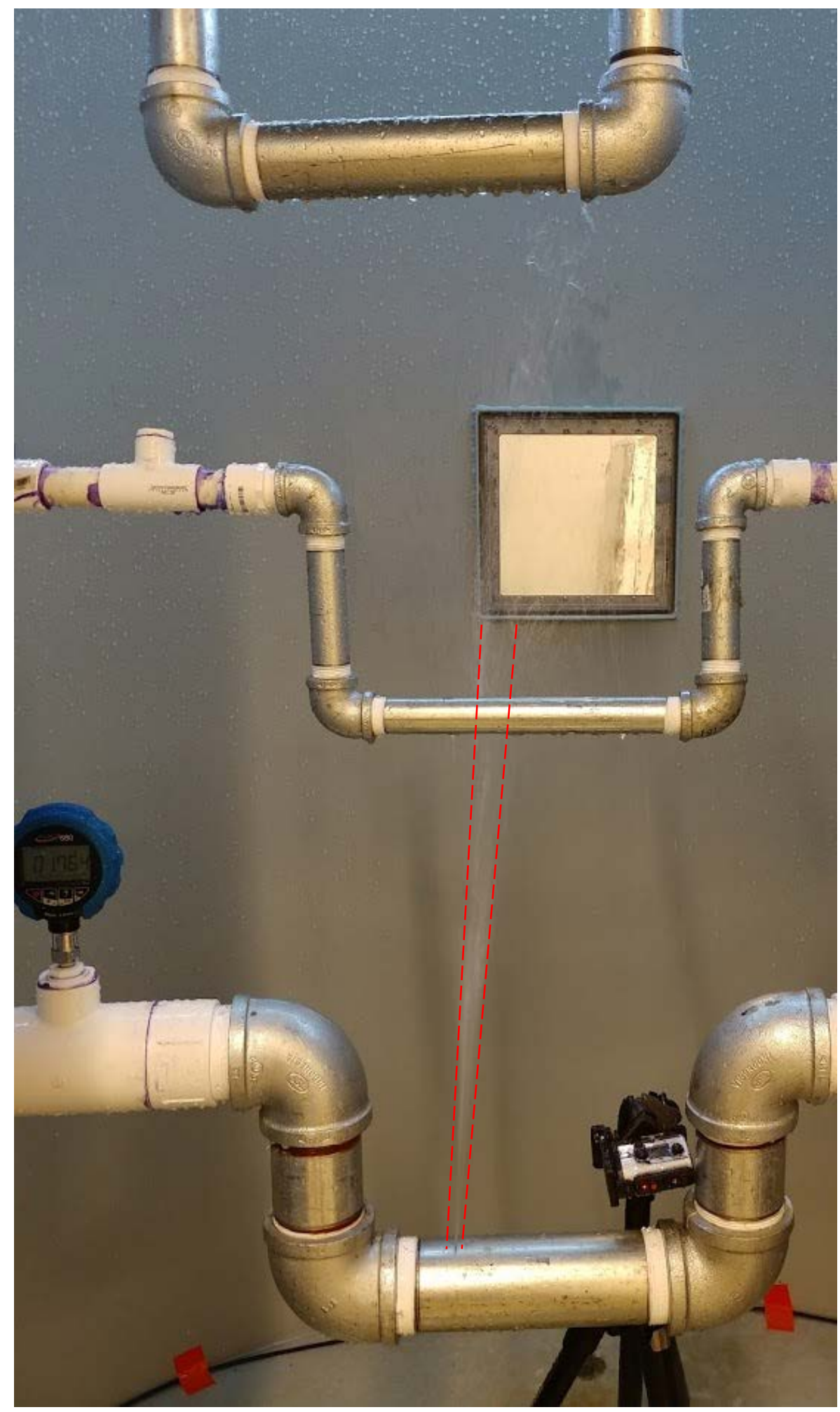

Figure 47. Type 2 leak from a circumferential leak.

The last type of leak identified is called Type 3, and is categorized by leaks with valves, or other large losses, downstream of the leaks. Due to the experiment design and setup, this category had the most representative runs. These types of runs are characterized by large leak rates, large velocities, low pressure drops across leaks, but large pressures at leaks as well. These types of leaks tended to be the most violent, and the mistiest. Figure 48 
shows a close view of a longitudinal crack, and Figure 49 a wide view. The wide view shows the leak hitting the back of the PET and splashing up and around the tank similar to the Type 1 in Figure 37. Additionally, the Type 3 leak comes out more normal to the pipe than the Type 1 or Type 2. Figure 50 shows a Type 3 leak from a circumferential leak and Figure 51 a Type 3 from an elbow. Both figures show the water being sharper and mistier than the other types. The angle is also more normal to the pipe than Type 2 . This type of leak has the potential to be the most damaging to components. The high flow means more water leaving the pipes. The high velocity means the water will carry further and become mistier as it exits the pipe. The high pressure means the water leaves the pipe more a more normal angle, than other types and is more direct. Table 9 shows an example data from one valve configuration giving Type 3 leaks, which is the two downstream valves partially closed.

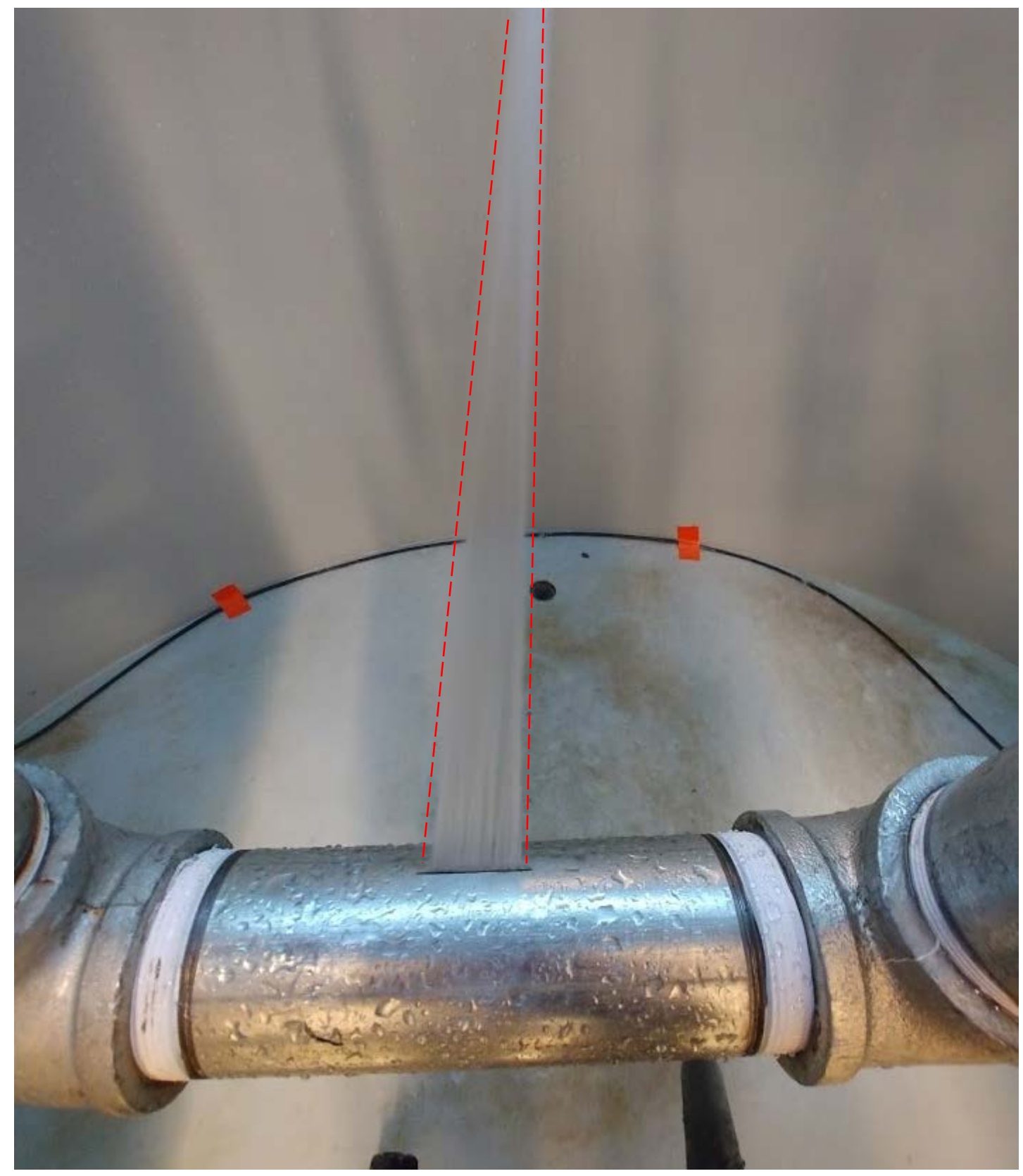

Figure 48. Type 3 leak from a longitudinal crack, close view. 


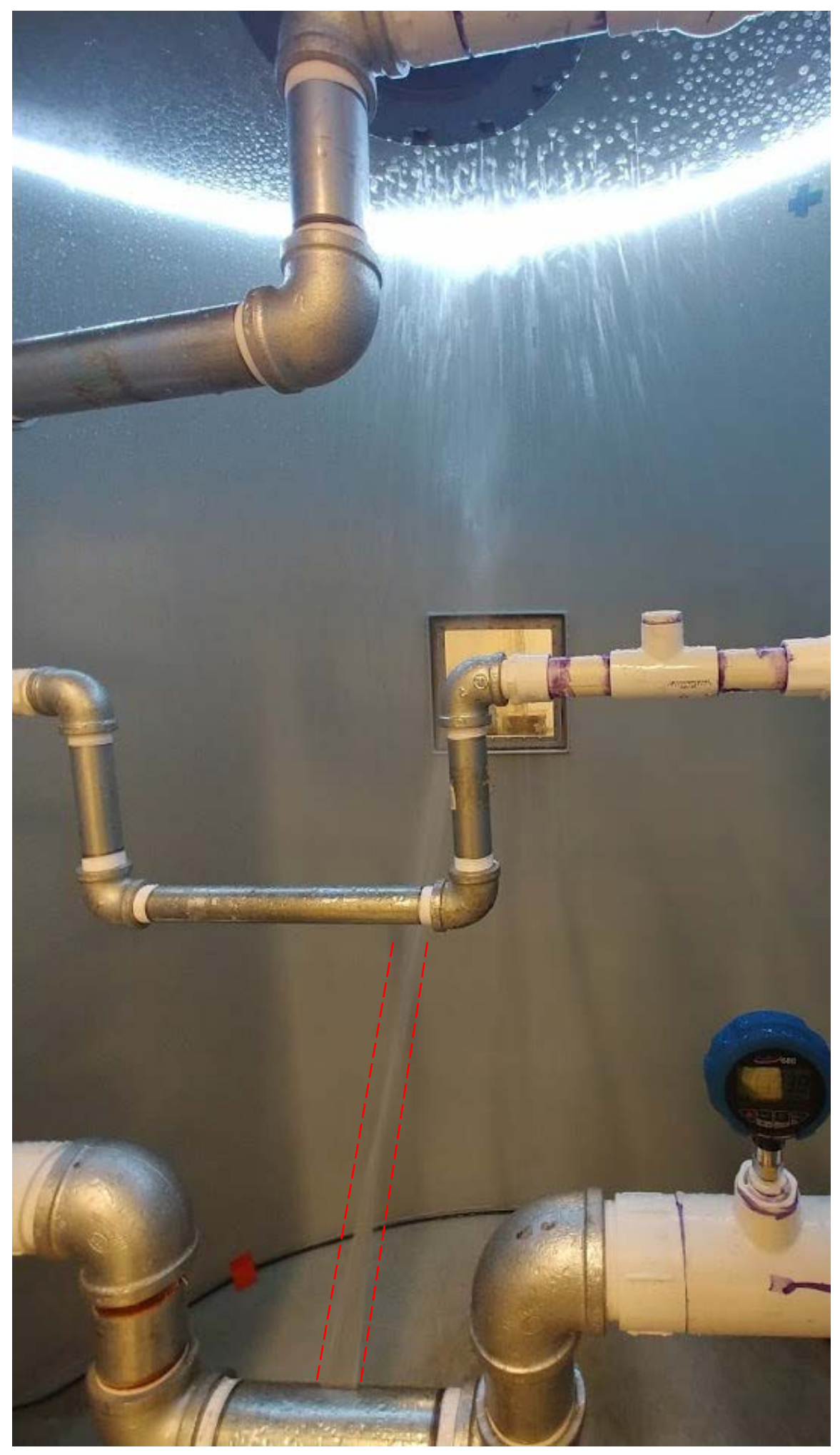

Figure 49. Type 3 leak from a longitudinal crack, wide view 


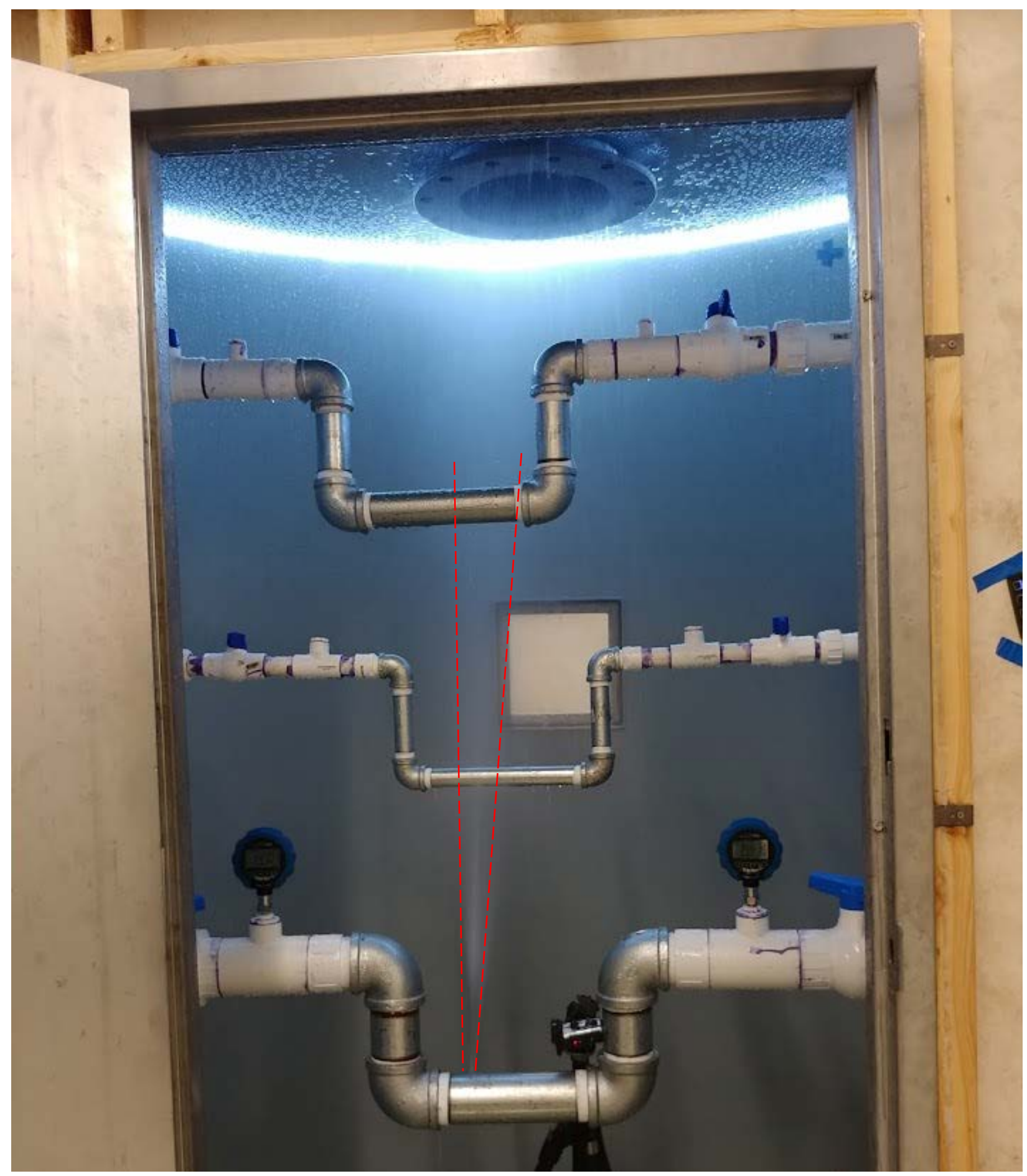

Figure 50. Type 3 leak from a circumferential leak. 


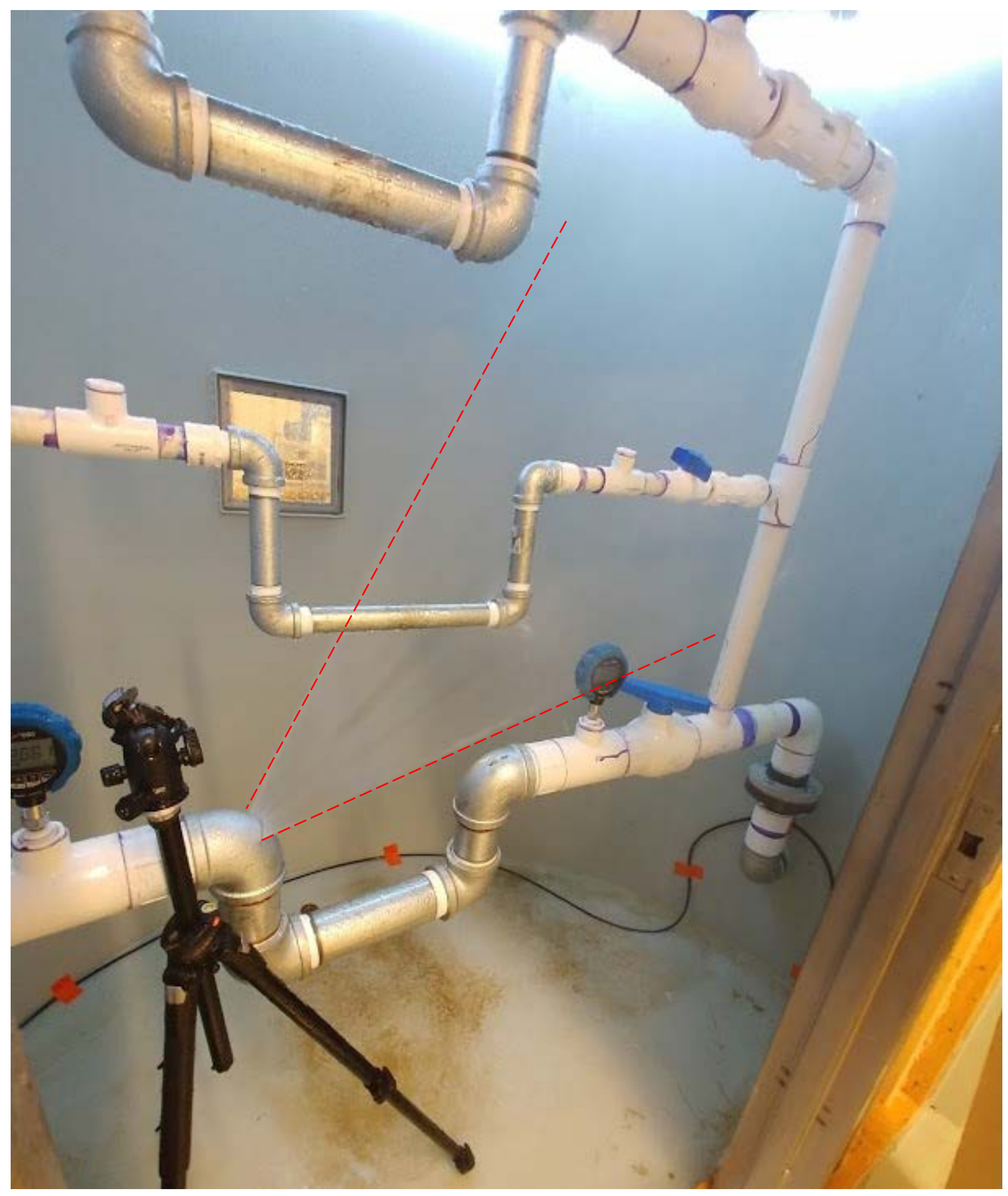

Figure 51. Type 3 leak from an elbow leak. 
Table 9. Type 3 Leak Data Example

\begin{tabular}{|c|c|c|c|c|c|c|}
\hline & $\begin{array}{c}\text { Leak rate } \\
\mathbf{( g p m )}\end{array}$ & $\begin{array}{c}\text { Error } \\
\mathbf{( g p m )}\end{array}$ & $\begin{array}{c}\text { Pressure at } \\
\text { Leak (psi) }\end{array}$ & Error (psi) & Velocity (ft/s) & Error (psi) \\
\hline Longitudinal & & & & & & \\
\hline 3-inch & 6.30 & 0.86 & 19.08 & 0.14 & 26.23 & 1.28 \\
\hline 2-inch & 4.6 & 0.59 & 10.51 & 0.19 & 14.35 & 0.64 \\
\hline 1-inch & 9.10 & 0.98 & 30.10 & 0.15 & 34.77 & 1.45 \\
\hline Circumferential & & & & & & 17 \\
\hline 3-inch & 7.35 & 0.72 & 22.71 & 0.17 & 28.45 & 1.19 \\
\hline 2-inch & 3.03 & 0.69 & 15.25 & 0.23 & 15.71 & 1.37 \\
\hline 1-inch & 6.95 & 0.46 & 24.14 & 0.19 & 72.61 & 2.84 \\
\hline Elbow & & & & & & 0.96 \\
\hline 3-inch & 9.34 & 0.74 & 21.49 & 0.16 & 27.08 & 1.29 \\
\hline 2-inch & 7.365 & 0.60 & 24.66 & 0.23 & 33.91 & 12.52 \\
\hline 1-inch & 7.44 & 6.00 & 30.06 & 0.16 & 59.02 & \\
\hline
\end{tabular}

Table 9 flow values are significantly higher than the two previous types, including one test with a velocity of $72 \mathrm{ft} / \mathrm{s}$. This was by far the largest velocity observed, due to the run also having one of the highest pressures. The same configuration with the longitudinal split had a comparable pressure at the leak, but due to the larger leak area, there is a larger pressure drop.

Results of these experiments demonstrate methods for describing different leak scenarios; longitudinal cracks, circumferential cracks, and cracks in elbows. Longitudinal cracks were shown to be directed streams, producing very little mist. The angle of the stream from the longitudinal crack is also dependent on the pressure in the pipe at the crack. Circumferential cracks produce a fan like effect for the spray, producing more mist than the longitudinal crack. The last type of crack in elbows was a combination of the other two, though it is more similar to the circumferential crack. This type typically had higher leak rates and velocities due to the changes in momentum present in elbows.

\section{SMOOTHED PARTICLE HYDRODYNAMICS}

Smoothed Particle Hydrodynamics (SPH) is a method for modeling fluid flow and is the main method being investigated for flooding event simulations. The goal of this work is to be able to either incorporate flooding failure data obtained from CFEL into an SPH code or to couple an SPH code with a risk assessment code that has the flooding failure data. By incorporating SPH and flooding failure data, it would allow for hypothetical flooding event simulations at NPPs to be modeled before a flooding event occurs. These simulations would allow the NPP risk analyst to determine what potential damage would be done if the flooding event were to occur. Additionally, the analysis would help determine if certain actions need to be taken that could reduce the damage of the flooding event. For flooding scenarios to be adequately modeled, SPH codes need to be validated against physical results to determine the accuracy of the code. Additionally, the optimum spacing of SPH particles needs to be used to ensure realistic simulation results.

To aid in the selection of particle spacing for SPH simulations, the SPH code Neutrino and RAVEN were coupled. RAVEN is an INL developed software tool that is a modular component that can be coupled with other systems [5]. It is capable of running external codes and performing parametric and probabilistic analysis based on 
the results of the external codes. RAVEN is written in Python and has a wide range of capabilities. Some of the capabilities of RAVEN include creation and use of reduced order models, forward propagation of uncertainties, advanced sampling methods, data post-processing, model parameter optimization, and data management. Additionally, RAVEN provides the framework for users to add new capabilities that will fit their needs. By coupling RAVEN and Neutrino, the goal is to use RAVEN's optimization framework and statistical analysis abilities to help determine the appropriate particle spacing for different scenarios. To achieve the Neutrino/RAVEN coupling, a Neutrino interface needed to be coded so RAVEN can run Neutrino.

The Neutrino interface is a python class consisting of five functions. Two of the functions are used to check the input file type, one function creates new Neutrino input files, one function generates the command to run Neutrino, and one function moves and converts the Neutrino output so RAVEN can interpret the results. The create new input function tells RAVEN to create new Neutrino input files by changing the particle interaction radius, or smoothing length, selected by RAVEN's sampling of distributions and changes the directories to where the results are stored. The generate command function tells RAVEN where to find the Neutrino executable and then how and what Neutrino input file to run. The finalize code output function edits and moves the Neutrino results so RAVEN can find the results and interpret them.

Once the Neutrino interface was created, a RAVEN input file was created to test the Neutrino/RAVEN coupling. The RAVEN input file consists of stating the Neutrino input file name, instructing RAVEN to use the Neutrino interface so RAVEN knows how to run Neutrino, creating different distributions and samplers, creating data objects for the results, and instructing RAVEN to plot the results. The Neutrino/RAVEN process consists of checking and reading the RAVEN input file, sampling the distribution to select new particle interaction radii, creating new Neutrino input with the sampled radii, generating and executing the commands for each Neutrino run, collecting the output from Neutrino, and plotting the results. Both Neutrino and RAVEN are installed on a local computer.

To show the Neutrino/RAVEN coupling, a simple example was created. The simple example consists of a rigid box, block of fluid, and a measurement field. Figure 52 shows the layout of the simple example for the Neutrino/RAVEN coupling.

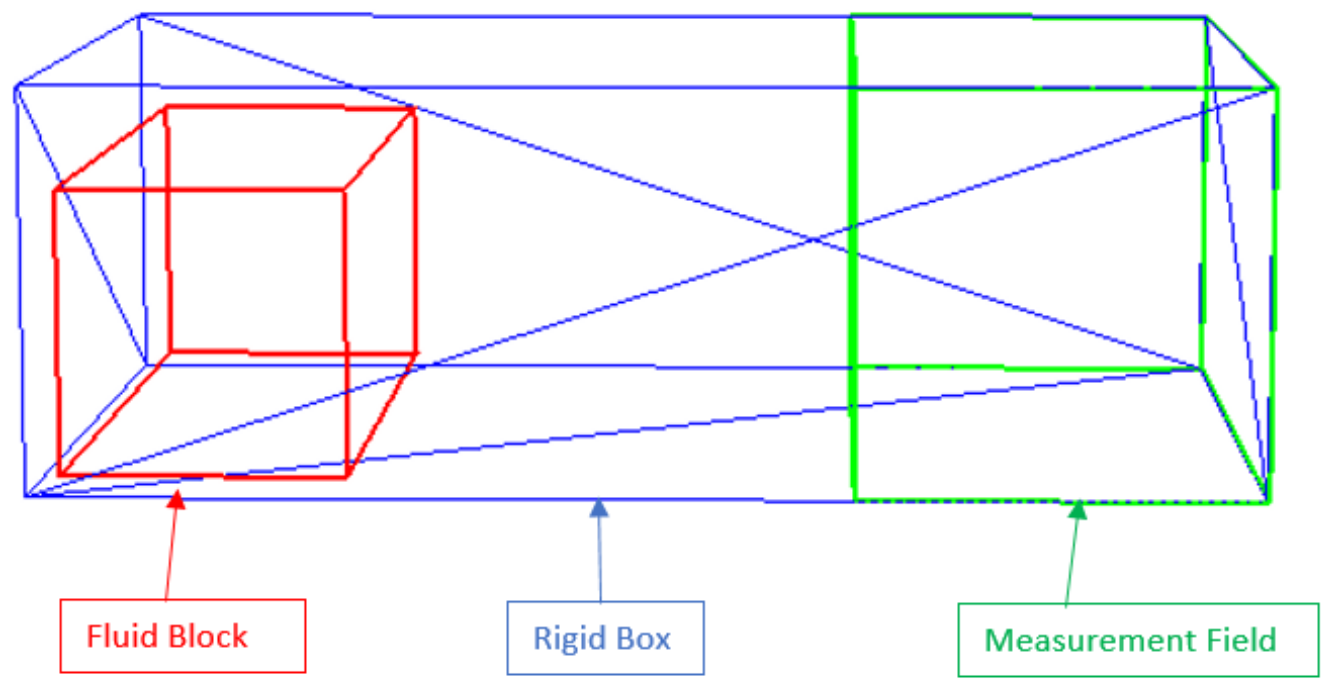

Figure 52: Neutrino/RAVEN simple example layout.

For the example, the block of fluid will collapse and flow throughout the rigid box. The measurement field will then measure the average height of the fluid inside the field. RAVEN will run this simulation five times for different particle interaction radii/smoothing length. Recall that the particle spacing is half the value of the 
particle interaction radii/smoothing length in Neutrino. Therefore, if the smoothing length is $1 \mathrm{~m}$, then the particle spacing is $0.5 \mathrm{~m}$. The smoothing length, and therefore particle spacing, will be sampled via Monte Carlo sampling from a uniform distribution ranging from 0.02 to 0.2 . The particle spacing will therefore range between 0.01 and 0.1. Figure 53 shows the average water height vs. time for different smoothing lengths. The color map on the right of the plot represents the colors for the different smoothing lengths.

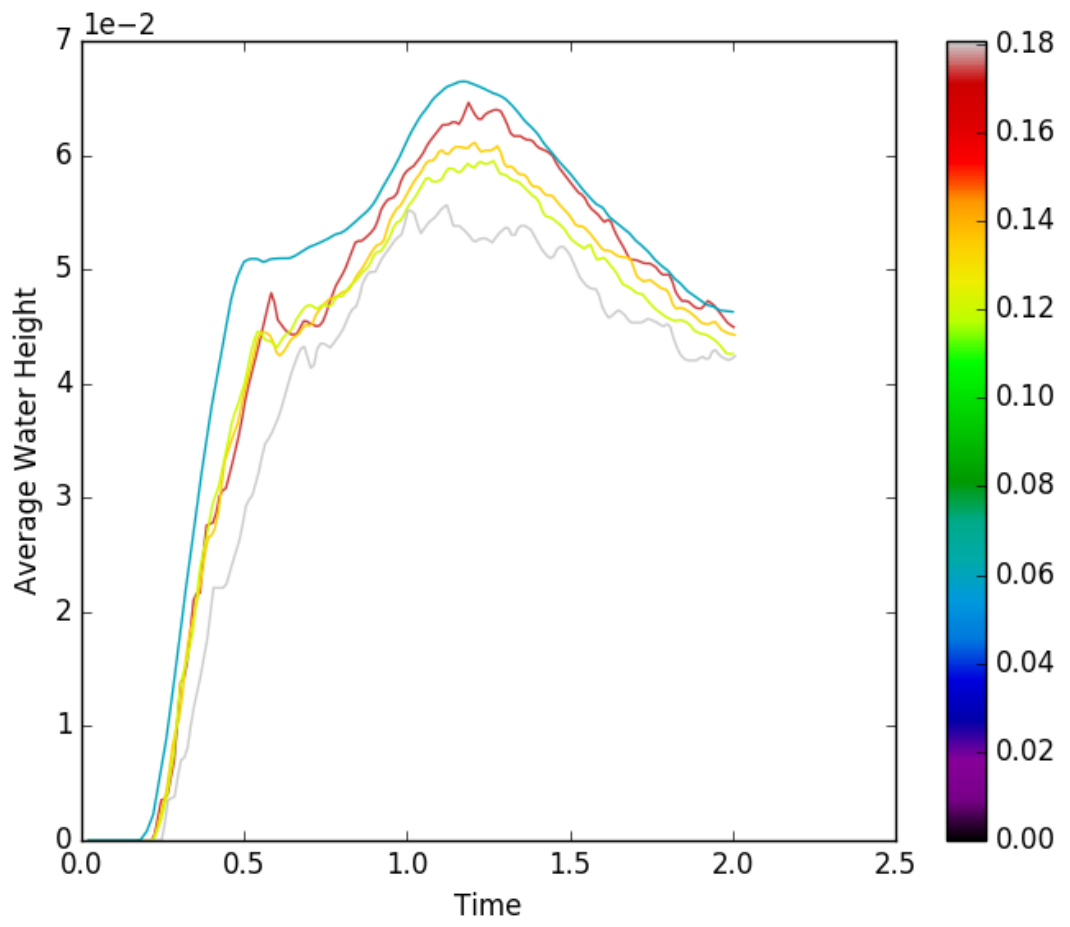

Figure 53: Neutrino/RAVEN simple example plot.

The plot shows that as the smoothing length decreases, the average water height measurement becomes smoother. This makes sense given the fact that a smaller smoothing length means a smaller particle spacing and therefore more particles for the measurement. Additionally, as the particle spacing becomes smaller, the average water height does not change as drastically when more particles move into the measurement field resulting in a smoother plot. The Neutrino/RAVEN coupling allows for a more efficient approach since it can change the Neutrino input, simulate multiple runs, and plot the results in one command. With the Neutrino/RAVEN coupling, research will be conducted to determine a protocol/optimizer for selecting the appropriate particle spacing for flooding simulations. Possible research entails incorporating a brute force convergence optimizer into RAVEN, simulating a wide variety of open channel flow and closed conduit flow models in Neutrino, analyzing the measurements obtained from the Neutrino models, analyzing the arrangement of spheres, and combining all of these analyses to create a protocol/optimizer for selecting the particle spacing.

Research is also being conducted at CFEL to compare SPH simulations using Neutrino for a series of physical experiments. The experimental setup consisted of a 1 in by 6 in by 12 in steel slab resting upright in the center of the flume and was subjected to known flow rates of water. The flow rates were recorded and increased until the slab fell over. The goal of the experiments is to determine the minimum flow rate that causes the steel slab to fall over which is identified as a failure event. Two configurations of the benchmark experiment were tested. Figure 54 depicts the horizontal orientation of the steel slab during one of the benchmark runs of the experiment and Figure 55 depicts the vertical orientation of the steel slab. 


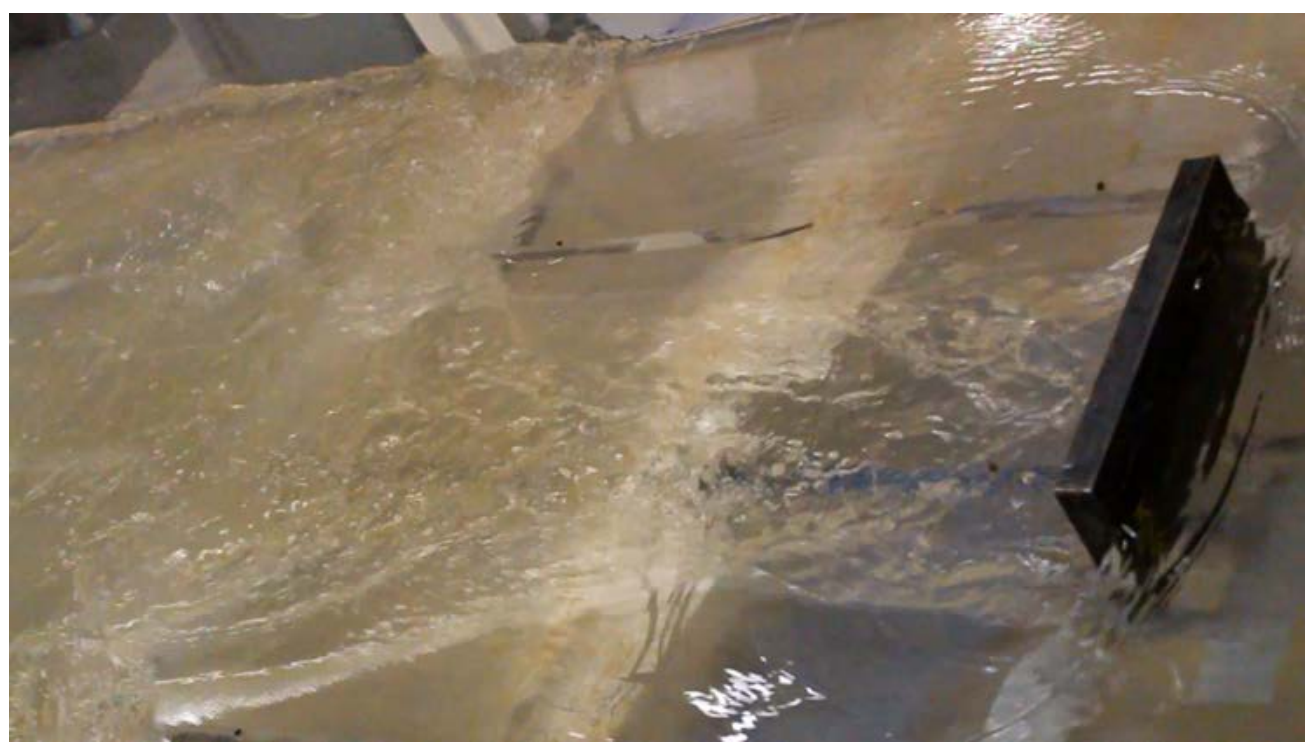

Figure 54. Horizontal orientation of benchmark experiment.

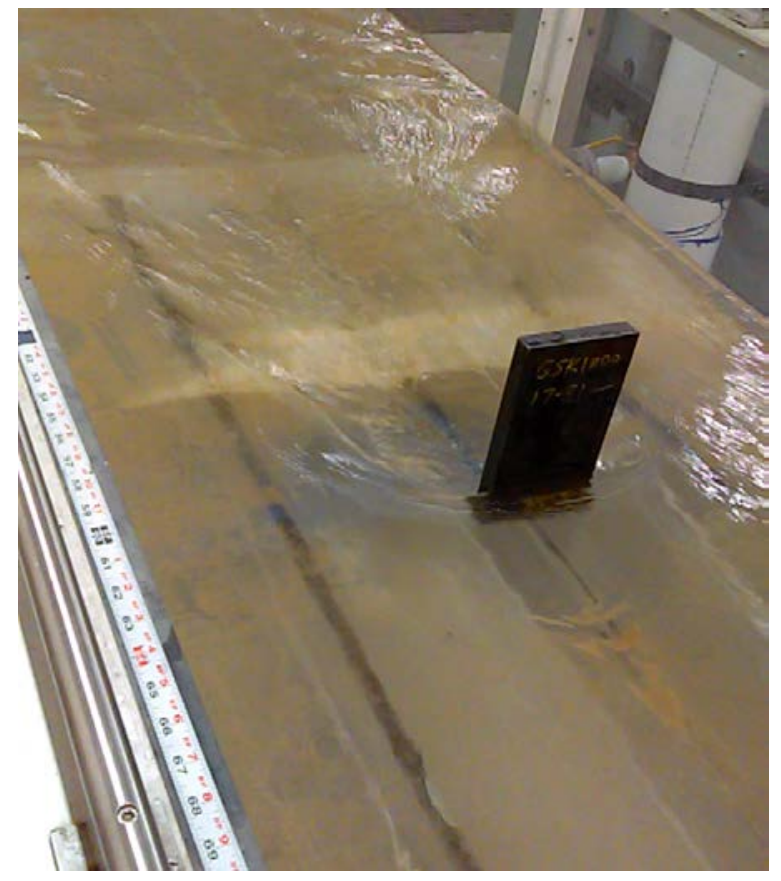

Figure 55. Vertical orientation of benchmark experiment.

Both configurations of the benchmark experiment have been completed. SPH simulations using Neutrino are being conducted for both configurations as well. Figure 56 shows the setup for the horizontally oriented simulation in Neutrino. 


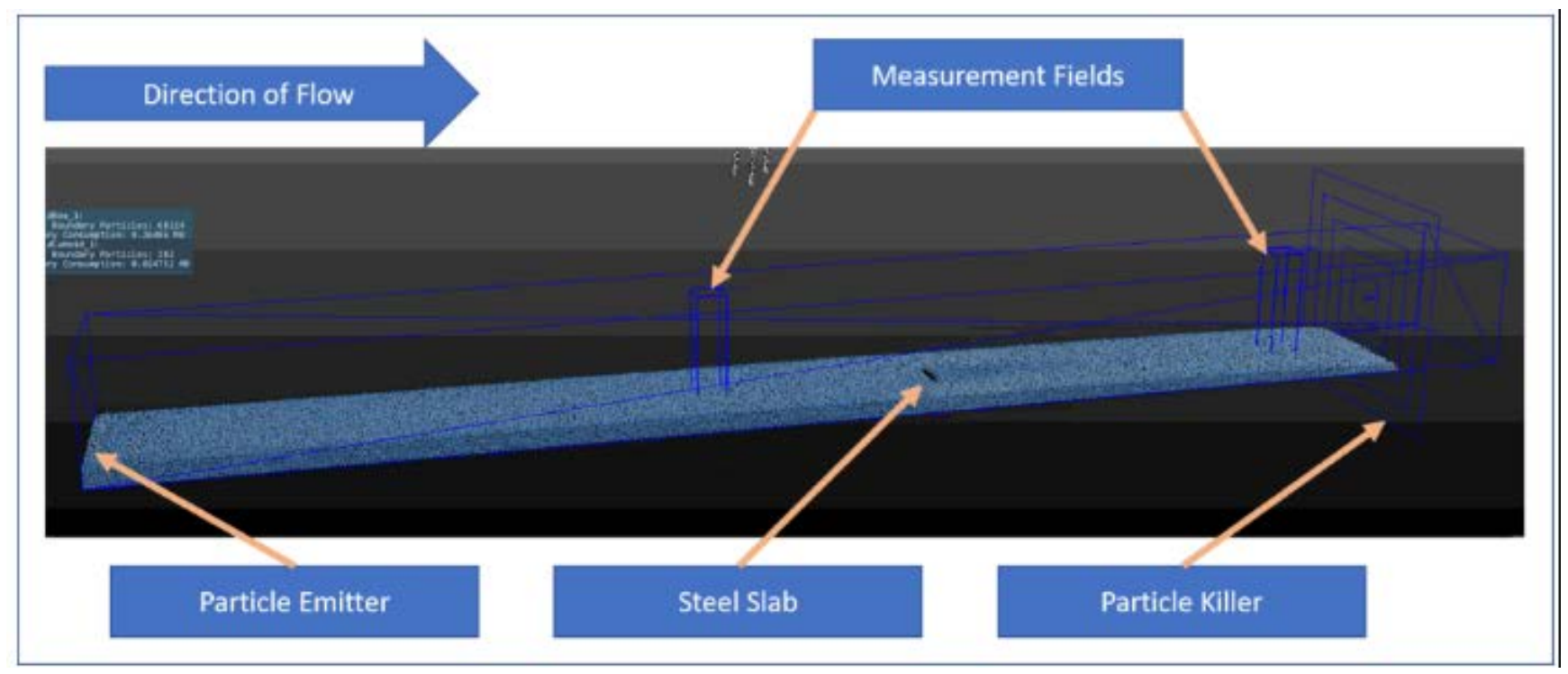

Figure 56. Neutrino SPH simulation for the horizontal orientation experiment.

Results of the physical experiments and preliminary simulation results have been compared. The flow rates needed to cause the steel slab to fall were greater in the experiments than in the simulations. Additionally, there were cases of translation in the SPH simulations, which did not occur in the physical experiment, where the slab slid across the bottom of the flume without falling over. The friction factor between the slab and the flume in the Neutrino simulation was increased to eliminate the translation occurrence.

Future work to improve the simulation result includes decreasing the particle spacing. A decrease in the particle spacing may yield results that are closer to those observed in the physical experiments. Ultimately, the goal of this comparison is to determine the validity of simulating dynamic rigid bodies in Neutrino.

\section{BAYESIAN REGRESSION FRAGILITY MODEL}

A flooding fragility model was developed using the door test data. For each of the experiments, data was collected for tank water depth (D), flow rate (F), and water temperature (T) as shown in Table 10. A failure (1) was assigned to tests where the damage to a door was permanent and the leakage area increased in a short amount of time. Success $(0)$ is defined when an equilibrium state was reached between the flow rate and leakage rate.

Table 10 Hollow Core Door Test Data

\begin{tabular}{|c|c|c|c|}
\hline Depth(in) & Flow Rate(gal/min) & Temp(F) & Failure \\
\hline 23.2 & 291.5 & 66 & 0 \\
\hline 20.8 & 292.5 & 67 & 0 \\
\hline 42.3 & 292.5 & 66 & 1 \\
\hline 21.1 & 297 & 68 & 0 \\
\hline 24.2 & 294.5 & 67 & 0 \\
\hline 35.4 & 292.5 & 67 & 1 \\
\hline 40.8 & 291 & 68 & 1 \\
\hline 38.9 & 294 & 68 & 1 \\
\hline
\end{tabular}

Initial mathematical modeling used the binomial model to represent failure of a door installed in the PET during a raising water flooding event. This is a commonly used model for failure on demand. The key variable in 
the binomial model, the failure probability on demand $p$ and $n=1$ (only one door is potentially challenged during testing). In this model, $\mathrm{p}$ is a possible function of depth (D), flow (F), and temperature (T). The parameter $\mathrm{p}$ is constrained between 0 and 1 and logit relation is used for $\mathrm{p}$ :

$$
\operatorname{logit}(p)=\ln \left(\frac{p}{1-p}\right)
$$

The fragility model examined seven possibilities with each of the parameters alone driving the model to failure, a combination of two factors driving the model to failure, and a combination of all three factors driving the model to failure. These models are:

$$
\begin{aligned}
& \operatorname{logit}(p)=\text { intercept }+a D+b F+c T \\
& \operatorname{logit}(p)=\text { intercept }+a D \\
& \operatorname{logit}(p)=\text { intercept }+b F \\
& \operatorname{logit}(p)=\text { intercept }+c T \\
& \operatorname{logit}(p)=\text { intercept }+a D+b F \\
& \operatorname{logit}(p)=\text { intercept }+a D+c T \\
& \operatorname{logit}(p)=\text { intercept }+b F+c T
\end{aligned}
$$

A script for OpenBUGS, a Bayesian analysis software tool using Markov Chain Monte Carlo (MCMC) methods, was written for seven equations. Since there was small variation in the flow rates and temperatures between runs, the outcome of failure is random with respects to these variables. Therefore, there is predictive capability on temperature and flow rate and the model had to be reduced to the depth variable when logit (p) function was used. This script is shown in Table 11.

Table 11 OpenBUGS Script for Depth Model

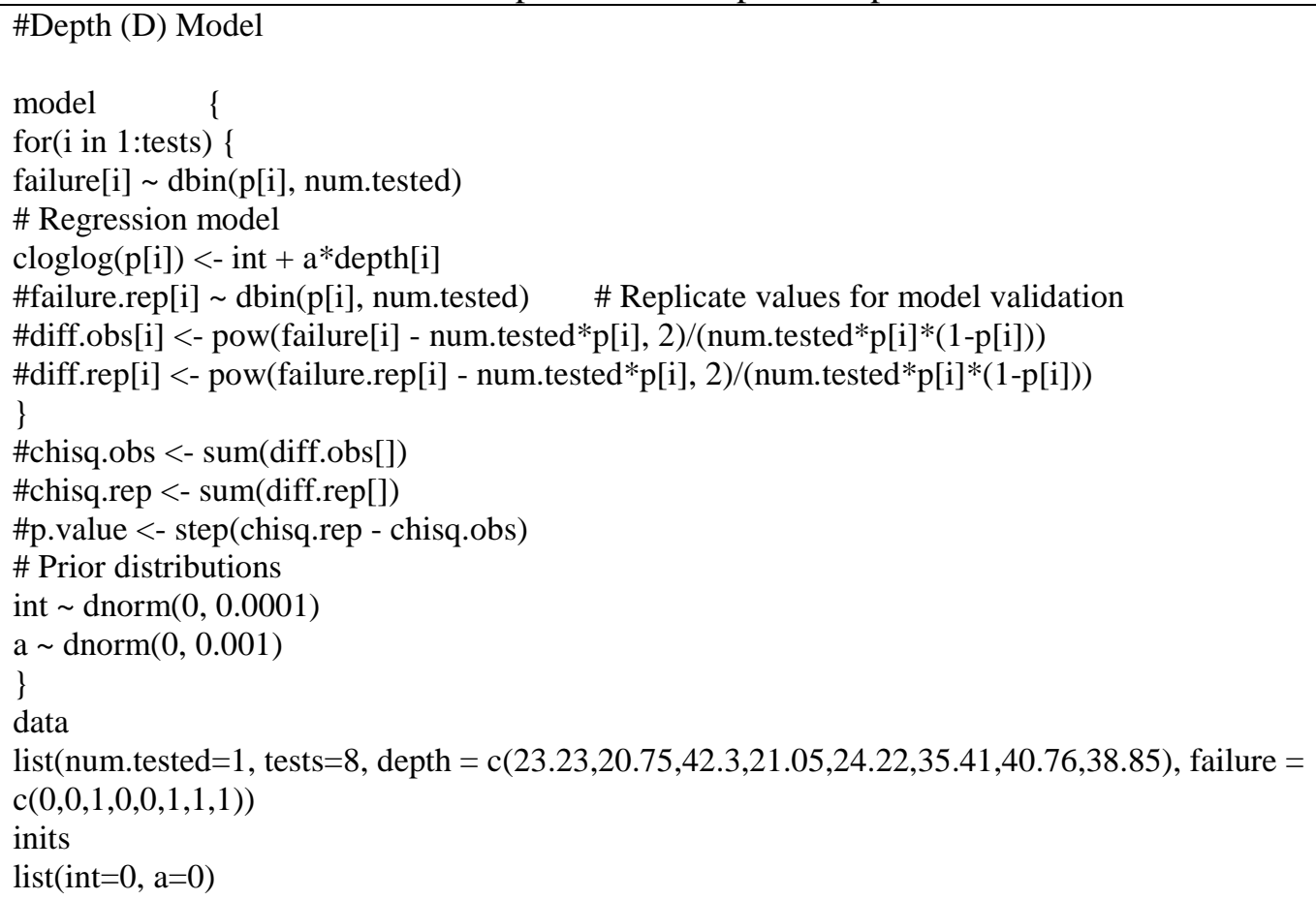


The results from running the script gives the depth coefficient and the intercept value as shown in Table 12. The Bayesian p-value was unavailable, because the number of samples obtained was insufficient.

Table 12 Results of the Depth Model

\begin{tabular}{|c|c|}
\hline Parameter & Mean Value \\
\hline intercept & 3.827 \\
\hline a (depth coefficient) & -110.2 \\
\hline Bayesian p-value & - \\
\hline
\end{tabular}

Since no conclusive results were obtained by using the logit function, the next section deals with possible functions that could potentially replace logit. Once the necessary coefficient values are established, $\operatorname{logit}(p)=$ intercept $+a D+b F+c T$, would be re-modeled for this case as:

$$
p=\frac{1}{e^{-(\text {intercept }+a D+b F+c T)}+1}
$$

The shortcoming with the logit function was recognized and an attempt was made to check other available link functions that could provide complete results. Available link functions supported by OpenBUGS are log, logit, cloglog and probit. They are defined as:

$$
\begin{gathered}
\log (p): \text { natural logarithm of } p \\
\operatorname{logit}(p)=\ln \left(\frac{p}{1-p}\right) \\
\operatorname{clog} \log (p): \text { complementary log log of } p \ln (-\ln (1-p)) \\
\operatorname{probit}(p): \text { inverse of standard normal cdf } p h i(p)
\end{gathered}
$$

\begin{tabular}{|c|c|c|c|c|c|}
\hline & variables & $\log$ & logit & cloglog & probit \\
\hline 1 & flow rate & $\bullet$ & $\checkmark$ & $\checkmark$ & $*$ \\
\hline 2 & depth & $\bullet$ & $\checkmark$ & $\checkmark$ & $\star$ \\
\hline 3 & temperature & $\checkmark$ & $\checkmark$ & $\checkmark$ & 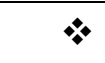 \\
\hline 4 & flow rate, depth & $\bullet$ & 0 & $\checkmark$ & $\%$ \\
\hline 5 & $\begin{array}{l}\text { flow rate, } \\
\text { temperature }\end{array}$ & $\bullet$ & $\checkmark$ & $\checkmark$ & $\nLeftarrow$ \\
\hline 6 & depth, temperature & $\bullet$ & 0 & $\checkmark$ & $\%$ \\
\hline 7 & $\begin{array}{l}\text { flow rate, depth, } \\
\text { temperature }\end{array}$ & $\bullet$ & 0 & $\checkmark$ & $*$ \\
\hline
\end{tabular}

Table 13 shows link functions behavior with designed flooding fragility models. The models with check mark indicate complete results available for intercept and coefficients. The symbols used in the table represents the errors faced while compilation and they are listed in Table 14.

Table 13 Models Responses to Link Function 
Table 14 Errors Observed While Using Different Link Functions

\begin{tabular}{|c|c|}
\hline Symbols & Observed Errors \\
\hline$\bullet$ & $\begin{array}{c}\text { Something went wrong in procedure; updater delayed. Sample in module } \\
\text { updater. }\end{array}$ \\
\hline 0 & Something went wrong in procedure. Sample in module update rejection. \\
\hline$*$ & Something went wrong in procedure node. Value in module graph probit \\
\hline
\end{tabular}

After testing available link functions, only cloglog script successfully ran for all seven models. Another way to predict the best model fit when the p-value is unavailable is using information criteria. This criterion measures the relative fit. A best model from the relative point of view may not be good from an absolute point of view. Two commonly discussed information criteria are the Bayesian Information Criteria (BIC) and Deviance Information Criteria (DIC). DIC is a measure of model fit that can be applied to Bayesian models and works when the parameter estimation is done using numerical techniques, such as Gibbs samplers. It is particularly useful in Bayesian model selection problems where the posterior distributions of the models have been obtained by MCMC simulation. DIC is a popular Bayesian analog of BIC. DIC has been recommended for selecting among the hierarchical models. A hierarchical model, also sometimes called multilevel model, has mutual dependence on the selected parameters that affect the modeling. The door test data shows clear interdependence on the factors that have been selected to affect regression modeling.

In OpenBUGS, Dbar is automatically monitored by the node called deviance. It is the posterior mean of the deviance and it requires no additional scripting. But mathematically, DIC is calculated as the sum of Dbar and $\mathrm{pD}$ :

$$
\begin{aligned}
& D I C=\text { Dbar }+p D \\
& p D=\text { Dbar }- \text { Dhat }
\end{aligned}
$$

where $\mathrm{pD}$ is the effective number of parameters and Dhat is deviance evaluated at posterior mean of parameter(s). DIC and even $\mathrm{pD}$ can be negative in some cases. DIC is usually negative when the density function $>1$. However, if $\mathrm{pD}$ turns out to be negative, DIC cannot be used. As a rule of thumb, the model with the smallest DIC usually indicates the best fitting model. For example, consider four models with DICs -11.5, -26, 10, 56. The second model is the best fit model because it has the smallest DIC among the others. It must also be noted that since DIC is a measure of relative fit, a model with the smallest DIC can still be a poor fit.

The scripts were re-written to change the link function from logit to cloglog. Table 15 shows the results obtained from running 100,000 samples and skipping the first 1000 samples. The best-fit model should be the first one (smallest DIC, of 0.02266) that has all the three parameters namely, depth, flow and temperature driving the model to failure. 
Table 15 Information on DIC and Other Parameters of the Seven Models

\begin{tabular}{|c|c|c|c|c|c|c|c|}
\hline Parameter & $\boldsymbol{D}, \boldsymbol{F}, \boldsymbol{T}$ & $\boldsymbol{D}$ & $\boldsymbol{F}$ & $\boldsymbol{T}$ & $\boldsymbol{D}, \boldsymbol{F}$ & $\boldsymbol{D}, \boldsymbol{T}$ & $\boldsymbol{F , T}$ \\
\hline Intercept & 5.653 & -108.0 & 3.468 & -6.659 & 3.289 & -2.621 & 9.64 \\
\hline $\begin{array}{c}\text { a } \\
\text { (depth } \\
\text { coeff) }\end{array}$ & 42.37 & 3.72 & - & - & 43.14 & 36.23 & - \\
\hline $\begin{array}{c}\text { b } \\
\text { (flow rate } \\
\text { coeff) }\end{array}$ & -7.109 & - & -0.01354 & - & -4.405 & - & 0.004231 \\
\hline $\begin{array}{c}\text { c } \\
\text { (temp } \\
\text { coeff) }\end{array}$ & 12.16 & - & - & 0.09177 & - & -16.06 & -0.1703 \\
\hline DIC & 0.02266 & 0.2804 & 12.74 & 12.36 & 0.02729 & 0.03374 & 14.23 \\
\hline
\end{tabular}

The initial mathematical modeling discussed in the previous background section used the binomial model to represent failure of a door installed in the PET during a raising water flooding event. This is a commonly used model for failure on demand. The key variable in the binomial model, the failure probability on demand $p$, became a possible function of the influencing factors water depth (D), flow rate (F), and temperature (T) of the water.

While trying to resolve some shortcomings of the OpenBUGS script use of the logit function, it was found that the binomial model was not running the complete number of samples assigned when a single component was put on demand $(n=1)$. While only one door was challenged during PET experiments, the logit script successfully ran all samples when $n$ was any value greater than one $(n>1)$. Since the binomial model was not performing when a single door was put on demand, other models were investigated. It should be noted that binomial might still be a useful aleatory model when the number of failure events is a positive integer in the internal $[0, n+1]$

Re-evaluating how demand was put on a door over a period of time while the water raised inside the tank, the next model interpretation could take a specified time period into account. The observed data will then be in the form of the door failure times in addition to depth, flow rate, and temperature. The failed hollow core door data is given in Table 16.

Table 16 Data on Inward Swinging Failures for Hollow Core Doors

\begin{tabular}{|c|c|c|c|}
\hline Time (min) & Flow Rate (gal/min) & Depth (in) & Temperature $\left({ }^{\circ} \mathbf{F}\right)$ \\
\hline 3.533 & 290.6 & 40.74 & 69.63 \\
\hline 2.667 & 294.07 & 38.77 & 69.02 \\
\hline 0.8667 & 411.25 & 32.28 & 62.18 \\
\hline 0.4667 & 472.89 & 43.34 & 63.49 \\
\hline
\end{tabular}

The simplest aleatory model for such data, when time is a random variable of interest, is an exponential distribution with unknown parameter $\lambda$. Before applying the possible influencing factors to the model, a standard exponential script was run using only the door failure times given in the first column of Table 16. The script for the exponential aleatory model in shown in Table 17. 
\#Exponential Model with No Environment; P-Value

model \{

for(i in $1: N)\{$

time[i] dexp(lambda)

time.rep[i] dexp(lambda)

\#Rank observed times a plicate times

time.ranked[i]<- ranked(time[],i)

time.rep.ranked[i]<-ranked(time.rep[],i)

\#Calculate components of Cramer-von Mises statictic for observed and replicated data

F.obs[i]<-cumulative(time[i], time.ranked[i])

F.rep[i]<-cumulative(time.rep[i], time.rep.ranked[i])

diff.obs[i]<-pow(F.obs[i] - $(2 * \mathrm{i}-1) /(2 * \mathrm{~N}), 2)$

diff.rep[i]<-pow(F.rep[i] - $(2 * \mathrm{i}-1) /(2 * \mathrm{~N}), 2)$

\}

\#Calculate distribution of Cramer-von Mises statistic for observed and replicated data

CVM.obs $<$-sum(diff.obs[])

CVM.rep<-sum(diff.rep[])

p.value<-step(CVM.rep-CVM.obs)

\# Prior distributions

\#lambda dnorm $(0,0.0001)$

lambda dgamma(0.0001, 0.0001) \#Jeffreys prior for lambda

\}\}

data

list $($ time $=c(3.533,2.667,0.8667,0.4667), \mathrm{N}=4)$

inits

list(lambda=1)

The parameter $\lambda$ has a specified prior distribution that represents the possible state of knowledge about possible values of $\lambda$ based on past or vendor provided information. Initially a Jeffreys non-informative prior was used in the script. For exponential data, the Jeffreys prior is like a gamma distribution with both parameters equal to zero. This is entered into OpenBUGS as $\operatorname{dgamma}(0.0001,0.0001)$ and an initial value most be provided for $\lambda$. Table 18 shows the results using the Jefferys prior distribution.

Table 18 Results for Standard Exponential Script with Jeffreys Prior

\begin{tabular}{|c|c|}
\hline Parameter & Mean Value \\
\hline$\lambda$ & 0.5309 \\
\hline Bayesian p-value & 0.6309 \\
\hline DIC & 15.13 \\
\hline
\end{tabular}

The prior distribution was then changed in the standard model script to a diffuse normal prior, as used in the previous binomial model. Running the script in Table 17 with the normal prior distribution $d n o r m(0,0.0001)$ gives the results found in Table 19. Comparing both the obtained p-value and DIC, the fitting capabilities of the model is improved using the normal prior. This comparison also illustrates the possible influences a prior distribution has on any given model. 
Table 19 Result of Standard Exponential Script with Diffuse Normal Prior

\begin{tabular}{|c|c|}
\hline Parameter & Mean Value \\
\hline$\lambda$ & 0.6617 \\
\hline Bayesian p-value & 0.6008 \\
\hline DIC & 14.93 \\
\hline
\end{tabular}

Further improvement could be made to the exponential model by including additional information, such as the environmental conditions. To determine what observable phenomena drive failure, $\lambda$ is not specified a prior distribution, but turned into its own model. The seven potential explanatory models are:

$$
\begin{aligned}
& \text { lambda }=\text { intercept }+f F \\
& \text { lambda }=\text { intercept }+d D \\
& \text { lambda }=\text { intercept }+t T \\
& \text { lambda }=\text { intercept }+f F+d D \\
& \text { lambda }=\text { intercept }+f F+t T \\
& \text { lambda }=\text { intercept }+d D+t T \\
& \text { lambda }=\text { intercept }+f F+d D+t T
\end{aligned}
$$

The OpenBUGS script for the last equation, which includes all three factors as explanatory variables, is shown in Table 20 and includes the Bayesian model check, p-value. Diffuse normal priors were used for the coefficient. It should be noted that while initial values were provided, the chain still contained uninitialized variables, and OpenBUGS was allowed to generate these values. 
Table 20 OpenBUGS Script for Exponential Aleatory Model with Bayesian p-value Calculation

\#Exponential Model Using Flow Rate (F), Depth (D), and Temperature (T); P-Value

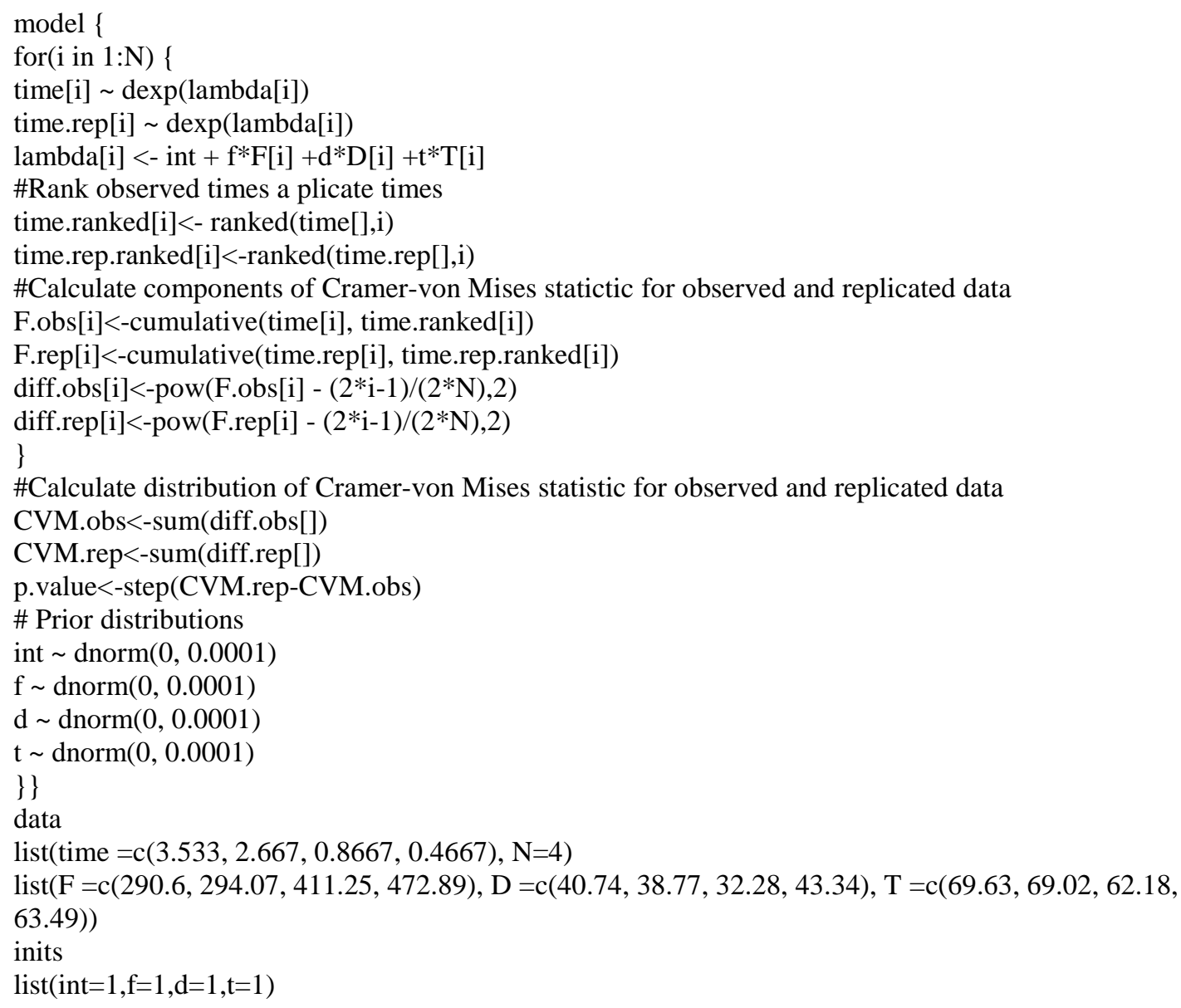

The model was analyzed with 1,000 burn-in iterations, followed by 10,000,000 iterations for parameter estimation. The code was run with the same number of samples as outlined with the binomial model background. The results for each coefficient, in addition to p-value and DIC, are summarized in Table 21. 
Table 21 Summary of Coefficient Estimates, p-value, and DIC for Exponential Model for Hollow Core Doors

\begin{tabular}{|c|c|c|c|c|c|c|c|}
\hline Parameter & $\boldsymbol{F}$ & $\boldsymbol{D}$ & $\boldsymbol{T}$ & $\boldsymbol{F , D}$ & $\boldsymbol{F}, \boldsymbol{T}$ & $\boldsymbol{D}, \boldsymbol{T}$ & $\boldsymbol{F}, \boldsymbol{D}, \boldsymbol{T}$ \\
\hline Intercept & -42.92 & -0.0402 & 10.59 & -16.44 & 43.58 & 2.82 & -10.92 \\
\hline $\begin{array}{c}\text { f } \\
\text { (flow rate } \\
\text { coeff) }\end{array}$ & 0.1488 & - & - & 0.1619 & 0.4927 & - & 0.2927 \\
\hline $\begin{array}{c}\text { d } \\
\text { (depth } \\
\text { coeff) }\end{array}$ & - & 2.49 & - & -0.7446 & - & -0.1429 & -0.185 \\
\hline $\begin{array}{c}\text { t } \\
\text { temp } \\
\text { coeff) }\end{array}$ & - & - & -0.1453 & - & -1.426 & 0.06877 & -0.9524 \\
\hline p-value & 0.8624 & 0.4689 & 0.3427 & 0.04328 & $6.533 \mathrm{E}-5$ & 0.1931 & 0.1705 \\
\hline DIC & 41.02 & 15.61 & 12.82 & 56.35 & 200.6 & 14.24 & 108.2 \\
\hline
\end{tabular}

The Bayesian p-value for the depth model is better than the value of 0.6008 from the standard exponential model. Taking into consideration the environment at failure improved the model without over-fitting the observed data. However, with calculated Bayesian p-values of 0.8624, 0.3427, 0.04328, 6.533E-5, 0.1931, and 0.1705 for the other respective six model equations are far enough from the ideal value of 0.5 , it is worth looking for a different aleatory model. Changing the model could give better predictive ability and there are several alternatives to the exponential model.

While the exponential distribution is an appropriate model in some situations, the analysis for door testing could require more complex aleatory models, such as the lognormal and Weibull distributions. The following alternative distributions have two parameters instead of a single one for exponential. In some cases, either of these parameters can be turned into a model in order to represent different test environments.

A lognormal aleatory model has two unknown parameters, commonly denoted by $\mu$ and $\sigma$. The shape of the distribution is fixed with the median determined by $\mu$ and the other parameter, $\sigma$, determines the spread of the distribution. This model could possibly be improved by including the additional testing environment information. The parameter $\mu$ will capture these influences on the results.

The example OpenBUGS script shown in Table 22 implements a lognormal aleatory model, time $[i] \sim \operatorname{dnorm}(\mu[i], \tau)$, with diffuse normal priors on the parameters of the $\mu$ model. Note that $\tau$ is equal to $1 /$ variance or $1 / \sigma^{2}$. The model was analyzed with 1,000 burn-in iterations, followed by 10,000,000 iterations. The posterior distributions of the parameters are summarized, along with Bayesian p-value and DIC, in Table 23. 
Table 22 OpenBUGS Script for Lognormal Model

\#Lognormal Model Using Flow Rate (F), Depth (D), and Temperature (T); P-Value

model \{

for(i in $1: N)\{$

time[i] dlnorm(mu[i], tau)

time.rep[i] dlnorm(mu[i], tau)

$\mathrm{mu}[\mathrm{i}]<-$ int $+\mathrm{f} * \mathrm{~F}[\mathrm{i}]+\mathrm{d} * \mathrm{D}[\mathrm{i}]+\mathrm{t} * \mathrm{~T}[\mathrm{i}]$

time.ranked[i]<- ranked(time[],i)

time.rep.ranked[i]<-ranked(time.rep[],i)

F.obs[i]<-cumulative(time[i], time.ranked[i])

F.rep[i]<-cumulative(time.rep[i], time.rep.ranked[i])

diff.obs[i]<-pow(F.obs[i] - $(2 * \mathrm{i}-1) /(2 * \mathrm{~N}), 2)$

diff.rep[i]<-pow(F.rep[i] - $(2 * \mathrm{i}-1) /(2 * \mathrm{~N}), 2)$

\}

CVM.obs<-sum(diff.obs[])

CVM.rep<-sum(diff.rep[])

p.value<-step(CVM.rep-CVM.obs)

tau $<$ - pow(sigma, -2$)$

sigma $\sim \operatorname{dunif}(0,10)$

\# Prior distributions

int $\sim \operatorname{dnorm}(0,0.0001)$

$\mathrm{f} \sim \operatorname{dnorm}(0,0.0001)$

$\mathrm{d} \sim \operatorname{dnorm}(0,0.0001)$

$\mathrm{t} \sim \operatorname{dnorm}(0,0.0001)$

Table 23 Summary of Coefficient Estimates, p-value, and DIC for Lognormal Model for Hollow Core Doors

\begin{tabular}{|c|c|c|c|c|c|c|c|}
\hline Parameter & $\boldsymbol{F}$ & $\boldsymbol{D}$ & $\boldsymbol{T}$ & $\boldsymbol{F}, \boldsymbol{D}$ & $\boldsymbol{F}, \boldsymbol{T}$ & $\boldsymbol{D}, \boldsymbol{T}$ & $\boldsymbol{F}, \boldsymbol{D}, \boldsymbol{T}$ \\
\hline Intercept & 4.222 & 0.6652 & -14.29 & 4.159 & 3.4 & -14.49 & 20.51 \\
\hline $\begin{array}{c}\text { f } \\
\text { (flow rate } \\
\text { coeff) }\end{array}$ & -0.01059 & - & - & -0.01066 & -0.00992 & - & -0.02009 \\
\hline $\begin{array}{c}\text { d } \\
\text { (depth } \\
\text { coeff) }\end{array}$ & - & -0.00857 & - & 0.002279 & - & -0.1123 & 0.08499 \\
\hline $\begin{array}{c}\text { t } \\
\text { temp } \\
\text { coeff) }\end{array}$ & - & - & 0.2213 & - & 0.008708 & 0.2903 & -0.2435 \\
\hline p-value & 0.5501 & 0.3276 & 0.4427 & 0.4239 & 0.457 & 0.4063 & 0.4403 \\
\hline DIC & 1.69 & 20.25 & 13.51 & 12.37 & 10.59 & 13.64 & 12.86 \\
\hline
\end{tabular}

From the lognormal consideration, the flow rate model and the combined flow rate and water temperature model are closest to the ideal value 0.5 . The flow rate over-predicted the observed data, while flow rate and temperature under-predicted by approximately the same margin. The flow rate also has the smallest DIC in this consideration, 1.69 versus 10.59, so it would be selected under this criterion. A DIC of 1.69 is also substantially lower than the smallest DIC obtained from the exponential distribution.

Like the lognormal distribution in the previous section, the normal distribution is characterized by the same two parameters, $\mu$ and $\sigma$. Therefore; the OpenBUGS script will be similar to Table 22 with the influencing 
factors applied to $\mu$, but the aleatory model is input as time $[i] \sim d$ norm $(\mu[i], \tau)$. Table 24 summarizes the results from running the normal distribution script.

Table 24 Summary of Coefficient Estimates, p-value, and DIC for Normal Model for Hollow Core Doors

\begin{tabular}{|c|c|c|c|c|c|c|c|c|}
\hline Parameter & $\boldsymbol{F}$ & & $\boldsymbol{D}$ & $\boldsymbol{T}$ & $\boldsymbol{F}, \boldsymbol{D}$ & $\boldsymbol{F}, \boldsymbol{T}$ & $\boldsymbol{D}, \boldsymbol{T}$ & $\boldsymbol{F}, \boldsymbol{D}, \boldsymbol{T}$ \\
\hline Intercept & 7.464 & & 0.3359 & -22.34 & 4.426 & 5.122 & -19.76 & -2.937 \\
\hline $\begin{array}{c}\text { f } \\
\text { (flow rate } \\
\text { coeff) }\end{array}$ & -0.01521 & - & - & -0.01577 & -0.01399 & - & -0.01127 \\
\hline $\begin{array}{c}\text { d } \\
\text { (depth } \\
\text { coeff) }\end{array}$ & - & 0.03984 & - & 0.0836 & - & -0.0773 & 0.02541 \\
\hline $\begin{array}{c}\text { t } \\
\text { temp } \\
\text { coeff) }\end{array}$ & - & & - & 0.3666 & - & 0.02865 & 0.3731 & 0.1207 \\
\hline p-value & 0.4464 & & 0.3345 & 0.4181 & 0.4082 & 0.446 & 0.3995 & 0.4144 \\
\hline DIC & 11.11 & & 20.76 & 11.81 & 16.12 & 15.29 & 16.06 & 16.05 \\
\hline
\end{tabular}

The Bayesian p-values 0.4464, 0.3345, 0.4181, 0.4082, 0.446, 0.3995, and 0.4144 are all reasonable choices, with all models slightly under-predicting the failure time. From this consideration, the flow rate model would be selected just above the flow rate and temperature model. Next looking at the calculated DIC, the flow rate model also has the smallest DIC, 11.11, so it would also be selected under this criterion.

The Weibull distribution is another alternative aleatory model for random durations, with two unknown parameters denoted as $\beta$ and $\alpha$. Unlike the lognormal or normal distribution, were the shape of the distribution is fixed, $\beta$ is a shape parameter. If $\beta$ equals 1 , the Weibull distribution reduces to the exponential distribution. The second parameter, $\alpha$, is a scale parameter. OpenBUGS uses a slightly different parameterization with the scale parameter as $\lambda=\alpha^{-\beta}$.

Allowing either parameter to be turned into a model and applying the influencing environment data could potentially improve upon this model. Therefore, two scripts for the Weibull distribution were created which alternated between one parameter capturing the influence of testing information and the other parameter being given a prior distribution and monitored by OpenBUGS. The example script shown in Table 25 sets $\beta$ as its own model, $\beta[i]$, and a prior to $\lambda$. Note that in order to run the code, the prior for the monitored Weibull parameter was set to a Jeffreys non-informative prior. The results of Table 25 script is compiled in Table 26, and the results from the alternate script that switched the roles of $\beta$ and $\lambda$ are given in Table 27. 
Table 25 OpenBUGS Script of a Weibull Distribution with Influence on the Beta Parameter \#Weibull Model (Beta) Using Flow Rate (F), Depth (D), and Temperature (T); P-Value

model \{

for(i in 1:N) \{

time[i] dweib(beta[i], lambda)

time.rep[i] dweib(beta[i], lambda)

beta[i] <- int $+\mathrm{f} * \mathrm{~F}[\mathrm{i}]+\mathrm{d} * \mathrm{D}[\mathrm{i}]+\mathrm{t} * \mathrm{~T}[\mathrm{i}]$

\#Rank observed times and duplicate times

time.ranked[i]<- ranked(time[],i)

time.rep.ranked[i]<-ranked(time.rep[],i)

\#Calculate components of Cramer-von Mises statictic for observed and replicated data

F.obs[i]<-cumulative(time[i], time.ranked[i])

F.rep[i]<-cumulative(time.rep[i], time.rep.ranked[i])

diff.obs[i]<-pow(F.obs[i] - $(2 * \mathrm{i}-1) /(2 * \mathrm{~N}), 2)$

diff.rep[i]<-pow(F.rep[i] - $(2 * \mathrm{i}-1) /(2 * \mathrm{~N}), 2)$

\}

\#Calculate distribution of Cramer-von Mises statistic for observed and replicated data

CVM.obs $<$-sum(diff.obs[])

CVM.rep<-sum(diff.rep[])

p.value<-step(CVM.rep-CVM.obs)

\# Prior distributions

lambda dgamma $(0.0001,0.0001)$

int $\sim \operatorname{dnorm}(0,0.0001)$

$\mathrm{f} \sim \operatorname{dnorm}(0,0.0001)$

$\mathrm{d} \sim \operatorname{dnorm}(0,0.0001)$

$\mathrm{t} \sim \operatorname{dnorm}(0,0.0001)$

\}\}data

list (time $=\mathrm{c}(3.533,2.667,0.8667,0.4667), \mathrm{N}=4)$

$\operatorname{list}(\mathrm{F}=\mathrm{c}(290.6,294.07,411.25,472.89), \mathrm{D}=\mathrm{c}(40.74,38.77,32.28,43.34), \mathrm{T}=\mathrm{c}(69.63,69.02,62.18,63.49))$

inits

list(lambda=1,int=1,f=1,d=1,t=1)

Table 26 Summary of Coefficient Estimates, p-value, and DIC for Weibull Model with Environment Impact on Beta parameter for Hollow Core Door

\begin{tabular}{|c|c|c|c|c|c|c|c|}
\hline Parameter & $\boldsymbol{F}$ & $\boldsymbol{D}$ & $\boldsymbol{T}$ & $\boldsymbol{F , D}$ & $\boldsymbol{F}, \boldsymbol{T}$ & $\boldsymbol{D}, \boldsymbol{T}$ & $\boldsymbol{F}, \boldsymbol{D}, \boldsymbol{T}$ \\
\hline Intercept & -79.1 & 10.24 & -0.2844 & -68.59 & -83.05 & 3.459 & -87.1 \\
\hline $\begin{array}{c}\text { f } \\
\text { (flow rate } \\
\text { coeff) }\end{array}$ & 0.275 & - & - & 0.3009 & 0.2567 & - & 0.31440 \\
\hline $\begin{array}{c}\text { d } \\
\text { (depth } \\
\text { coeff) }\end{array}$ & - & -0.20600 & - & -0.44280 & - & -0.17530 & -0.903 \\
\hline $\begin{array}{c}\text { t } \\
\text { temp } \\
\text { coeff) }\end{array}$ & - & - & 0.03484 & - & 0.1337 & 0.08585 & 0.4794 \\
\hline lambda & 0.56520 & 0.2376 & 0.2735 & 0.4022 & 0.5825 & 0.2099 & 0.282 \\
\hline p-value & 0.2897 & 0.3378 & 0.2965 & 0.1832 & 0.2857 & 0.3096 & 0.1203 \\
\hline DIC & 63.56 & 15.43 & 15.66 & 64.05 & 53.68 & 15.13 & 63.45 \\
\hline
\end{tabular}


Table 27 Summary of Coefficient Estimates, p-value, and DIC for Weibull Model with Environment Impact on Lambda Parameter for Hollow Core Door

\begin{tabular}{|c|c|c|c|c|c|c|c|}
\hline Parameter & $\boldsymbol{F}$ & $\boldsymbol{D}$ & $\boldsymbol{T}$ & $\boldsymbol{F , D}$ & $\boldsymbol{F}, \boldsymbol{T}$ & $\boldsymbol{D}, \boldsymbol{T}$ & $\boldsymbol{F}, \boldsymbol{D}, \boldsymbol{T}$ \\
\hline Intercept & -44.93 & 1.986 & 7.683 & -52.66 & -10.84 & 8.641 & -13.61 \\
\hline $\begin{array}{c}\text { f } \\
\text { (flow rate } \\
\text { coeff) }\end{array}$ & 0.1549 & - & - & 0.2924 & 0.5672 & - & 0.4226 \\
\hline $\begin{array}{c}\text { d } \\
\text { (depth } \\
\text { coeff) }\end{array}$ & - & -0.03097 & - & -0.78990 & - & -0.0128 & 0.2437 \\
\hline $\begin{array}{c}\text { t } \\
\text { (temp } \\
\text { coeff) }\end{array}$ & - & - & -0.1057 & - & -2.21 & -0.1099 & -1.71 \\
\hline beta & 2.384 & 1.034 & 1.693 & 1.82 & 2.144 & 1.532 & 2.273 \\
\hline p-value & 0.9767 & 0.4579 & 0.2679 & $2.36 \mathrm{E}-4$ & $2.51 \mathrm{E}-5$ & 0.2981 & 0.01096 \\
\hline DIC & 36.67 & 17.27 & 10.8 & 104 & 207.1 & 10.95 & 138.8 \\
\hline
\end{tabular}

For both Weibull considerations, the water depth model was closest to the target 0.5 , with the environment impact on $\lambda$ returning closer results to 0.5 . It is also of interest that the $\beta$ value of this model is 1.034 , within rounding of 1 were the Weibull reduces to the exponential distribution. Depth was also the best-fit model for the exponential distribution.

The following Bayesian p-values were closest to the ideal value, while still under-predicting the failure time, for each distribution: exponential, 0.4689; lognormal, 0.457; normal, 0.4464; Weibull $(\beta)$, 0.3378; Weibull $(\lambda), 0.4579$. These are all reasonable choices that divide between including depth information (exponential, Weibull) and flow rate information (lognormal, normal) in the modeling. The flow rate model returned the significantly smallest DIC criterion for all models with the lognormal distribution, justifying the additional complexity of distributions with more than one parameter and the inclusion of additional experimental information. Factoring in the environment at failure could enhance a model's reliability predictions.

\section{WAVE IMPACT SIMULATION DEVICE SECTIONAL MODEL}

As part of the research for CFEL, this section focuses on improvements in the existing numerical model for a Wave Impact Simulation Device (WISD) and to conduct a physical experimental study to help validate the concept. The WISD is an air driven wave generator capable of generating a wave traveling at $25.4 \mathrm{ft}$ per second that is $10 \mathrm{ft}$ high and $10 \mathrm{ft}$ wide. It uses ten similar sections, each with a depth of one foot, stacked one on top of the other. After the air pressure is activated, the wave produced by each section combines to form a 10-foot wave section. Initially, the gates hold the water at rest and open quickly after the air pressure is activated. The design is based on the characteristics of a numerically simulated 20-foot tsunami wave.

Tsunamis are one of the most catastrophic natural events, generating floods that have proven to be a probable threat to nuclear facilities in the past. The March 2011 tsunami at the Fukushima NPP has clearly exposed the risk associated with flooding incidents in NPP's. The goal of the WISD is to allow testing and collecting information that can be applied to risk modeling studies of NPP components and facilities.

Most wave generators around the world use large wave basins and flumes which are expensive to construct and require large experimenting areas. These wave generators use solitary wave equations to produce tsunamilike wave behavior, however, some researchers believe solitary waves underestimate the total energy of a tsunami. 
The WISD, instead of generating an artificial wave for impact testing, produces a high-velocity jet as an alternative to simulate a high-velocity wave impact. Using high-velocity jets instead of waves avoids this debate and can economize available resources at the same time.

FLOW-3D, a commercially available computational fluid dynamics (CFD) program which solves the Reynolds-averaged Navier-Stokes equations, was used to simulate tsunami waves and tsunami wave impacts. Tsunami waves with a height of 20 feet were simulated approaching a solid model, recording flow data such as peak impact forces, pressures, and velocities. This information was then used to direct the design of the WISD. The flow behavior of the tsunami wave was replicated in the numerical code using solitary wave assumptions. To provide a wide range of testing capability for wave impact tests, the maximum horizontal fluid velocity for a 20-foot tsunami wave, 25.4 feet per second, was the criteria used to meet CFEL requirements concluding Roberts's study on tsunami waves.

In addition, ten distinct designs were studied to generate a wave in the laboratory traveling at a maximum velocity of 25.4 feet per second. A primary criterion for the design was to maintain a near vertical wavefront to imitate a tsunami wave traveling up an inclined beach. Design $\mathrm{J}$ as the most viable WISD option for validation and further study. Design J used air pressure to provide the motive force for the water retained behind the bottomhinged gates. Figure 57 and Figure 58 show the isometric view of Design J and the resulting wave section, respectively.

Plots for the velocity of the wave versus time indicated the velocities are unsteady. However, one of the objectives for CFEL was to generate a steady wave that would conserve the maximum velocity of 25.4 feet per second for a considerable fraction of the total simulation. This study concentrates on developing a steady wave.

Different geometries, in terms of fluid depth and approach angle, are tested in quest of the best geometry to produce a steady velocity. To retain water in the storage tank, diverse options have been explored with and without gates. Furthermore, the results from the numerical simulations will be used in a physical model study to validate the concept. Results from the physical experimental study will be used to build a scaled down model of the WISD.

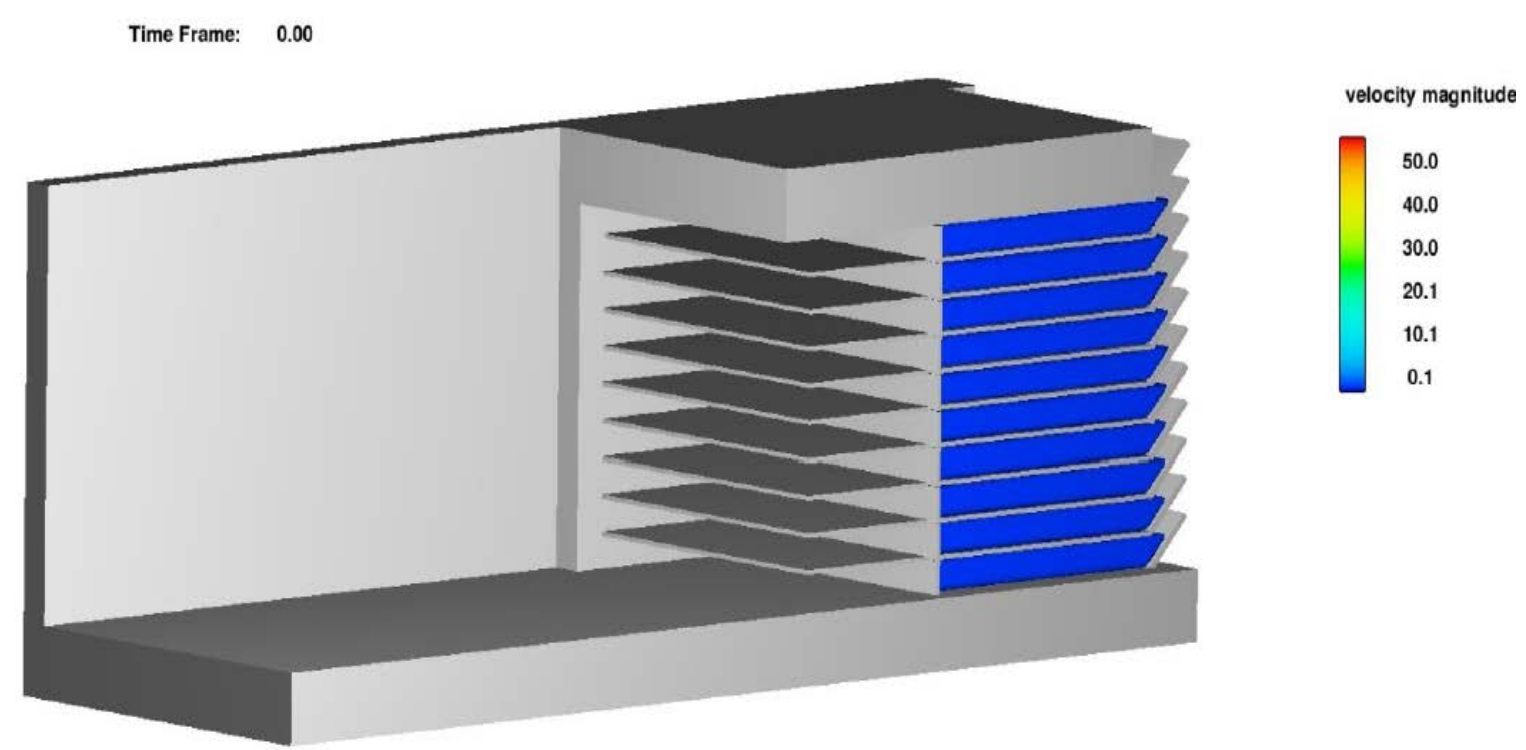

Figure 57: Wave Impact Simulation Device design. 


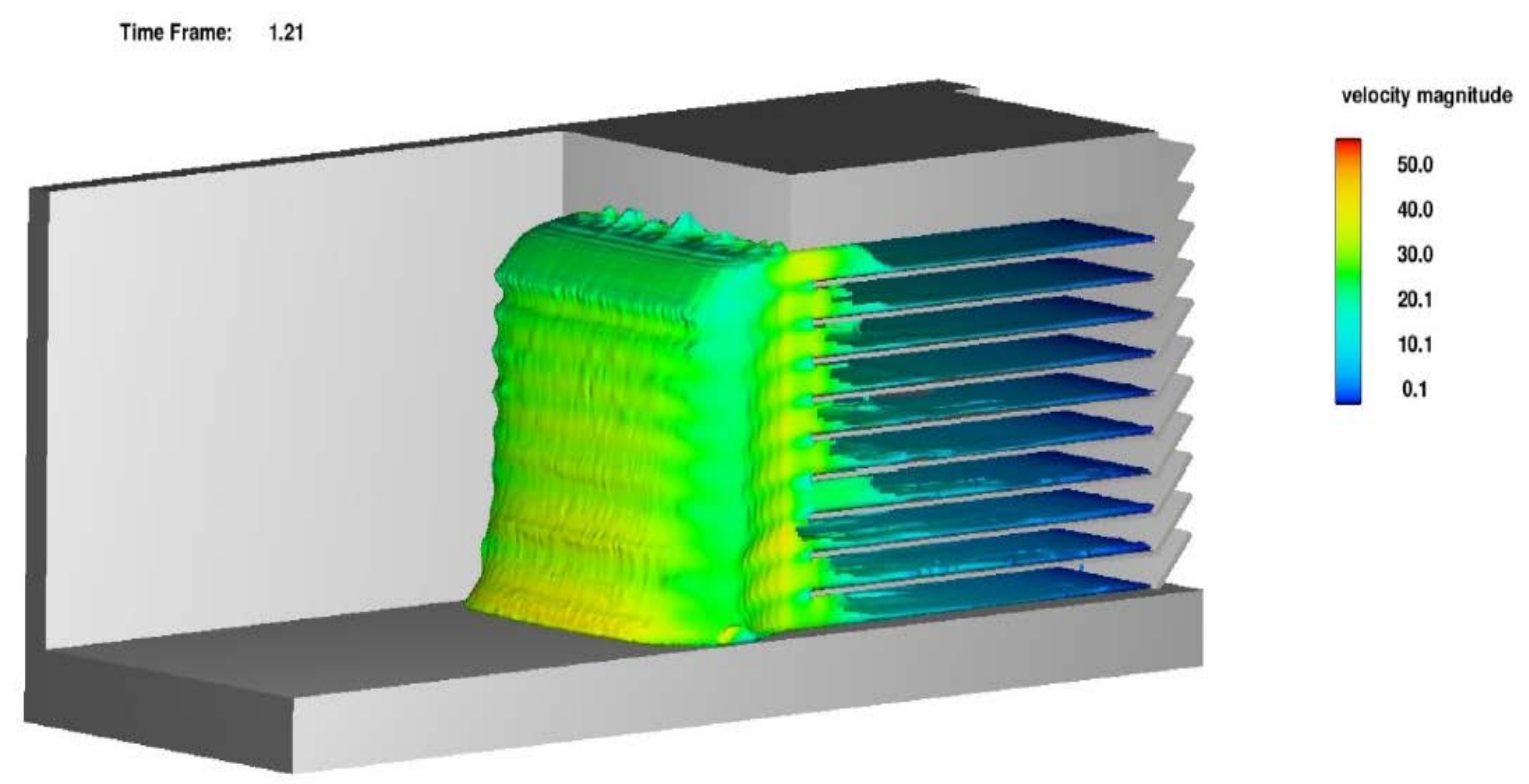

Figure 58: Vertical face wave front.

The scaled physical model of the prototype will be designed in a 1:5 scale ratio. The physical model will provide further insights of the selected design. Generating a 10-ft. high wavefront in a laboratory, conveying the energy of a tsunami wave, opens a new dimension of full-scale nuclear components and facilities testing under extreme flooding conditions. Most importantly, further improvements in the components and facilities can be developed to sustain such drastic events.

Significant research has been conducted to generate a laboratory scale wave with an objective to study the behavior, impacts, inundation flows and other aspects of an oceanic wave. Concentrating on the physical and numerical modeling of a tsunami-like wave, laboratory generation techniques and modeling methodologies were investigated. Fluid kinematics and air pressure variability were explored to generate a steady state fluid motion. Existing wave flumes and basins around the world are also briefly discussed in this section.

The foundation of ocean research is characterized by the identification of natural formation of waves and their behavior. Numerous wave theories have been developed to define the behavior of waves. Waves are characterized by their relative height and wavelengths. To describe these characteristics, the wave type that best represents the natural conditions is identified and represented by mathematical equations. The validity of these theories is limited to different ranges of wave parameters (height and wavelengths) and water depth. Stressing the limitations to generate a tsunami in a laboratory scale, researchers often use solitary waves to resemble tsunami waves. A solitary wave has no trailing or preceding waves associated with it and has a long wavelength.

The extensive use of coastal and offshore structures makes wave impact on shorelines important. The complexity involved with these wave-induced flow urges the use of empirical coefficients to expand the theoretical formulation. Broadly used calculations for forces generated by the wave is developed with the assumption that wave forces can be expressed as the sum of a drag force and an inertia force.

Numerical modeling is widely gaining popularity for its efficient nature and ability to model complex fluids problems. There are several methods for modeling and computing numerical models; some of which are Finite Volume Method, SPH method and Finite Element Method. The CFD code, FLOW-3D, which uses a Finite Volume Method, is used for this study. Because it uses a finite volume method in a regular hexagonal grid, the form of the employed discrete equations is similar to discrete equations in finite difference method. This numerical 
code is well known for modeling flows with free water surface and complex geometries. A unique post-processing tool named Flow-Sight has excellent abilities to portray the results of a simulation.

Most CFD codes depend on the subdivision of space into a grid of discrete volume elements in which average values of flow variables can be defined. FLOW-3D uses the simplest kind of grid composed of rectangular-shaped elements defined by a set of planes perpendicular to each of the coordinate axes generating a hexahedron around the domain. Difference equations are simpler in comparison to non-structured grids. Numerical approximations to partial differential equations include the rate of change of spatial and temporal values of physical quantities, which improves the accuracy associated with finite difference equations when uniform grid elements are used. However, it is difficult to accommodate complex geometries using a rectangular mesh limiting their usefulness.

Different rectangular mesh blocks can be used to define a domain using various cell sizes. Defining mesh planes at desired locations generate a variable spaced block allowing more efficiency in the gridding of complex geometric regions. Finer meshes can be embedded into a coarser mesh depending on the problem setup and precision of the desired result. These rectangular meshes could be efficiently used to open up memory and urges solver routines to basically run through a list of active grid elements to further save computational time.

To analyze, describe, and apply appropriate boundary conditions on a free surface, FLOW-3D uses the Volume-of-Fluid method. This method operates by determining the volume of fluid within each discretized cell. An empty cell receives a value of zero, and, a value of one is assigned if a cell is full. A free surface is represented in a cell with a value between 0 and 1 that correlates to the ratio of fluid volume to cell volume. FLOW-3D uses a grid porosity technique called Fractional Area/Volume Obstacle Representation method to define the solid geometries in 3D using computational cells. A porosity of zero is assigned to solid regions and a porosity of one is assigned to nonsolid regions. Like the VOF method, cells with both obstacle and flow volume are assigned a value per the ratio of the volume of the obstacle to the volume of the cell.

The boundary conditions defined by the user must accurately represent the physical occurrence of a flow. It is very important in every simulation as it directly affects the simulation results. Since a hexahedron is used to define the flow domain in Cartesian coordinates, there are six different boundaries on the mesh, in addition to the obstacle surface.

Generating a wave in a laboratory that characterizes a tsunami can be challenging with respect to multiple aspects. For example, the laboratory space available and the financial cost could be prohibitive. So, meticulous design and rigorous pre-testing are necessary. Numerical model study and physical experimental study would identify any defects in the design of the WISD.

Previous CFEL research resulted in the conceptual design the mechanism of the WISD. However, for better performance of the device, these questions remain unanswered: How should the air pressure be varied to produce a constant velocity in the jet? Which geometry, in terms of fluid entrance depth and approach angles, will yield a near vertical wave profile? Does a system with gates produce a near vertical wave profile, or does the system without gates yield a near vertical wave profile?

Using CFD simulations it was concluded that the best motive force to be used was air pressure over other options like hydraulically actuated pistons. This was determined by testing different designs of a general geometry for the WISD with the goal to produce a maximum velocity of 25.4 feet per second. A major advantage of using air pressure over another possible method to generate the motive force like pistons, was the flexibility to use different fluid depths by dividing it into discrete channels.

The motive force needs to provide a large force to initiate motion in water and then reduce to maintain a steady fluid velocity by overcoming the frictional forces. To reduce the computational time to compute a transient motive force, a sectional model was considered using only one section of the jet. In addition, modifications to the section were made, looking for the most effective arrangement to apply air pressure to displace the water by 
considering differently angled inlets and fluid depths. Table 28 shows the various simulations that were conducted in search of the optimum geometry. Parameters like wave profile and velocity were considered to compare the different geometries.

Table 28: Simulations Conducted in FLOW 3D

\begin{tabular}{|c|c|c|c|}
\hline Design No. & Design ID. & Inlet Angle (In degrees) & Fluid Depth (ft.) \\
\hline 1 & WD-45-1 & 45 & 1 \\
\hline 2 & WD-45-2 & 45 & 2 \\
\hline 3 & WD-45-3 & 45 & 3 \\
\hline 4 & WD-35-1 & 35 & 2 \\
\hline 5 & WD-35-2 & 35 & 3 \\
\hline 6 & WD-35-3 & 35 & 1 \\
\hline 7 & WD-25-1 & 25 & 2 \\
\hline 8 & WD-25-2 & 25 & 3 \\
\hline 9 & WD-25-3 & 25 & \\
\hline
\end{tabular}

3-D solid geometries of the WISD sectional model were designed in AutoCAD, and, a stereolithographic file format was used to import it into Flow-3D. A rectangular grid, with a mesh size of 0.02 feet defined the flow domain in Flow-3D which divides one foot into 50 cells. Grid convergence tests were not conducted in this study as it has already been tested in previous research. Figure 59 and Figure 60 show two of the designed listed in Table 28.

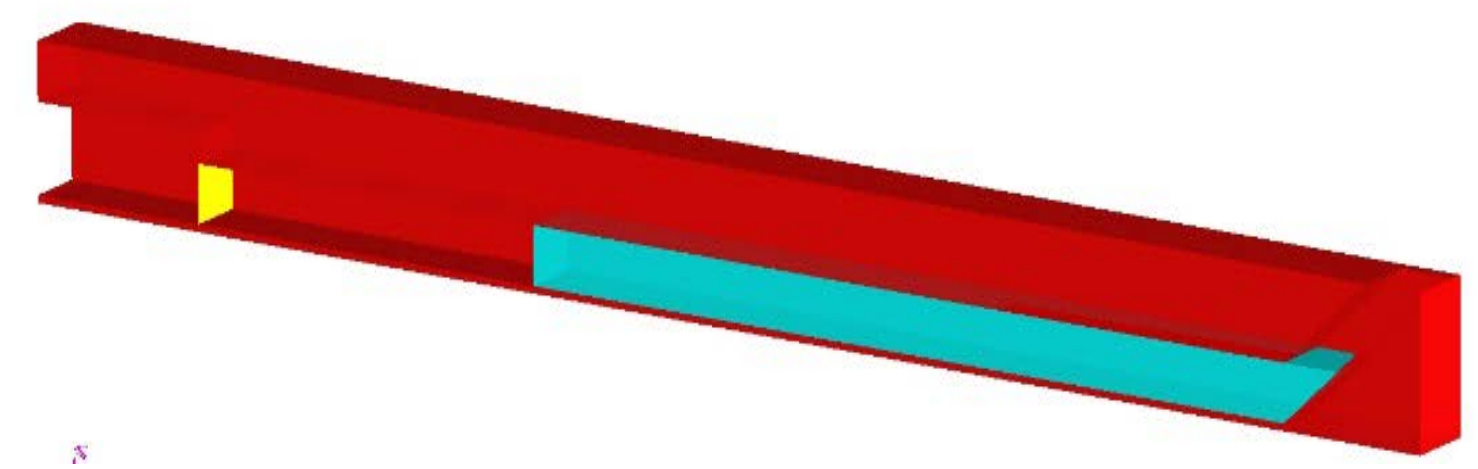

Figure 59: Sectional model Design 1, WD-45-1.

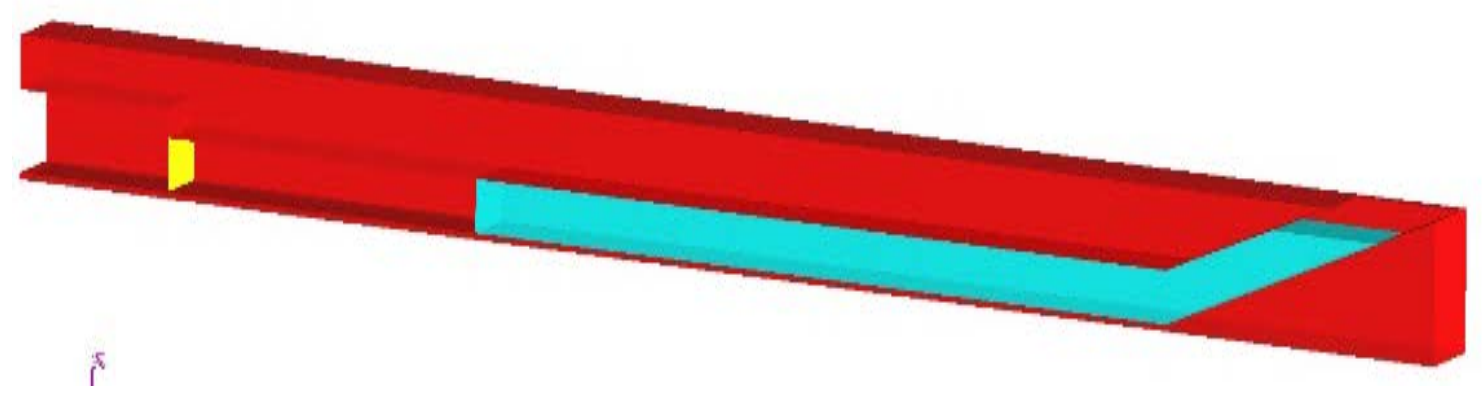

Figure 60: Sectional model Design 2, WD-25-2.

A bottom hinged watertight gate was placed downstream to retain the water in the reservoir. To measure the flow rate exiting the chamber, a baffle was placed at the same location for all arrangements. These numerical 
baffles do not impede or change the flow, rather they were used to better understand the fluid behavior. Immediately downstream of the baffle, the height of the channel was increased to replicate an open channel flow where the wave profile was observed. The motive air pressure was set to $625 \mathrm{psf}$. (lbs. $/ \mathrm{ft}^{2}$ ) for the simulations from an initial time, $\mathrm{t}=0$ seconds to 1.5 seconds. All the simulations behaved quite similar. Results show that the fluid would rapidly accelerate at $\mathrm{t}=0.8$ seconds to a maximum velocity without maintaining it.

Although these simulations provide useful information on the behavior of the motive force, one of the goals of the WISD is to have a constant velocity for a considerable portion of the total time frame, which did not occur. To modify the flow behavior, at approximately $t=0.8$ seconds, the motive air pressure was decreased sharply starting from $t=0.8$ seconds. This was applied to one simulation with an inlet depth of one foot and a 45 degrees angle. The varying inlet air pressure is shown in Table 29. The resulting velocity was fairly constant.

Table 29: Varying pressure boundary

\begin{tabular}{|c|c|}
\hline Time (secs.) & Pressure (psf.) \\
\hline 0 & 625 \\
\hline 0.3 & 625 \\
\hline 0.6 & 625 \\
\hline 0.8 & 625 \\
\hline 0.9 & 75 \\
\hline 1.2 & 50 \\
\hline 1.5 & 0 \\
\hline
\end{tabular}

Using the assumption that the water moves as a solid block through the conduit and applying general kinematic equations and Newton's second law of motion which are mentioned in the literature, it was determined that a pressure force of $2144.1 \mathrm{lbs}$. $/ \mathrm{ft}^{2}$ was required to accelerate the water to a velocity of 25.4 feet per second. This supplies the necessary inertial force to start the water moving. And, once the water "block" achieved the desired velocity, the only the force needed to maintain this velocity was a force to match the shear stress of the water acting on the structure walls.

Another important component for the WISD was the gates. These mechanical sections have to withstand the hydrostatic pressure from the retained fluid behind it with minimum possible leakage. Also, these gates had to be designed in such a way that at the exact moment when the air pressure would hit the water, they have to open quickly allowing the fluid to move. This principle was adopted so that the air pressure behind the fluid would not add forces on to the gate, and thinner gates could be used with strength enough to withstand the hydrostatic pressure of the resting water. These gates on opening were supposed to merge on to the plates underneath, without influencing the wave profile, so thick gates are not an option. A snapshot of the gate holding water is shown in Figure 61.

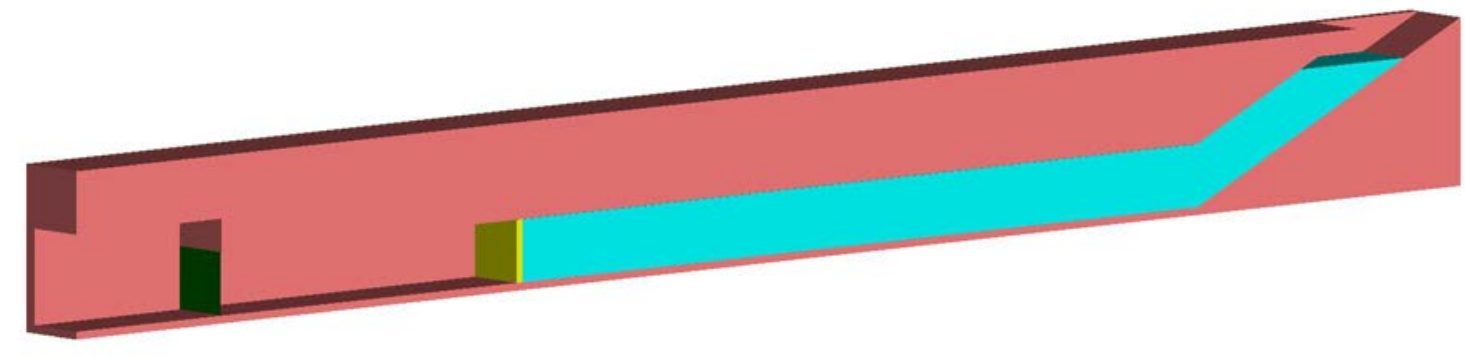

Figure 61: Gate (yellow) used for simulation. 
Using the general feature of FLOW-3D to assign the gate as a moving object, it was hinged at its bottom and was assigned to rotate at the gate axis in the flow direction. Figure 62 shows the gate opening on its bottom axis, allowing the fluid to move upon the action of air pressure. A motion was prescribed to the gate component manually, and directional limitations were assigned to the gate so that it would merge with the solid plate located below.

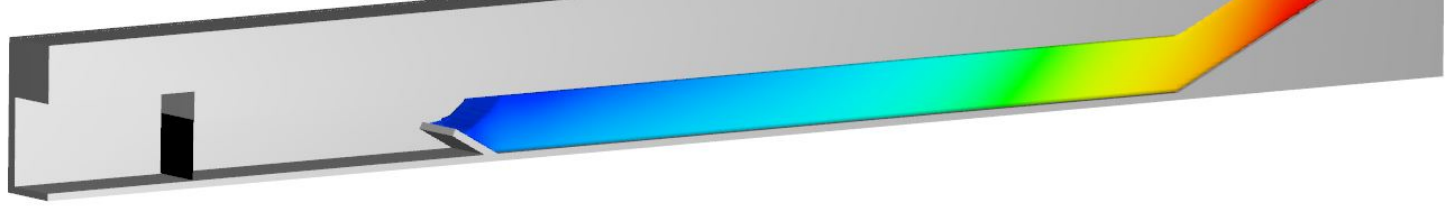

Figure 62: Representation of gate partially opening.

The gates used in the WISD produced excellent results, however, another option was also explored excluding the gate. For this case, a U-tube shape was used to retain the fluid and similar to the option with the gate, air pressure was used to apply the motive force. The inlet and outlet were inclined, and different angles and depths were adopted to produce a steady state water jet with a near vertical wave profile. It was necessary for the inlet and outlet to be inclined but not at 90 degrees, as a larger space would be required in the laboratory for construction of the full-scale prototype.

Similar to the models with the gate, 3-D solid geometries of the U-tube model were designed in AutoCAD, and, a stereolithographic file (STL) format was used to import it into FLOW-3D. A rectangular grid defined the flow domain with a mesh size of 0.02 feet. The pressure was applied through the $\mathrm{X}_{\min }$ boundary. Simulating these settings as a 2-D simulation, the adjacent boundaries, $\mathrm{Y}_{\min }$ and $\mathrm{Y}_{\max }$, were defined as symmetry boundaries and the top and bottom boundary, $\mathrm{Z}_{\min }$, and $\mathrm{Z}_{\max }$ were defined as walls with the downstream boundary, $\mathrm{X}_{\max }$, set as outflow to allow free flow out of the simulation. The $\mathrm{Z}_{\min }$ boundary was also defined as a wall. Table 30 provides a list of simulations that were conducted without gates in the WISD. Figure 63, Figure 64, and Figure 65 provide a side views of some two-dimensional WISD simulations without gates.

Table 30: Sectional Model Simulations Conducted Without Gates

\begin{tabular}{|c|c|c|c|}
\hline Design No. & Design ID & Inlet Angle (In degrees) & Fluid Depth (ft.) \\
\hline 10 & WD-45-1-WG & 45 & 1 \\
\hline 11 & WD-45-2-WG & 45 & 2 \\
\hline 12 & WD-35-1-WG & 35 & 1 \\
\hline 13 & WD-35-2-WG & 35 & 2 \\
\hline 14 & WD-25-1-WG & 25 & 1 \\
\hline 15 & WD-25-2-WG & 25 & 1 \\
\hline 16 & WD-55-1-WG & 55 & 1 \\
\hline 17 & WD-45-1-WG-1C & 45 & 1 \\
\hline 18 & WD-45-1-WG-2C & 45 & 1 \\
\hline 19 & WD-45-1-WG-3C & 45 & 1 \\
\hline 20 & WD-45-1-WG-PHY & 45 & 2 \\
\hline
\end{tabular}




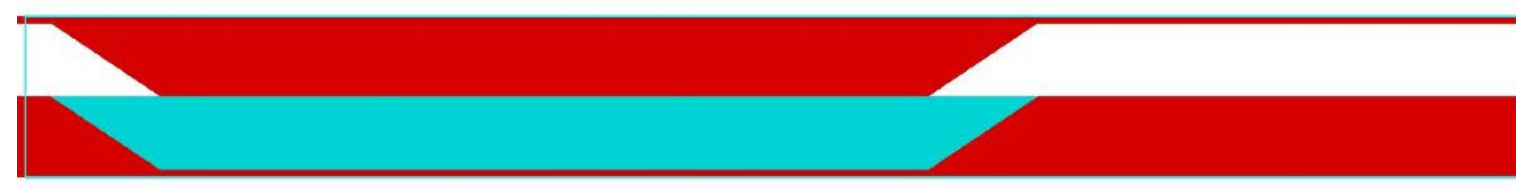

Figure 63: WD-45-1-WG (side view).

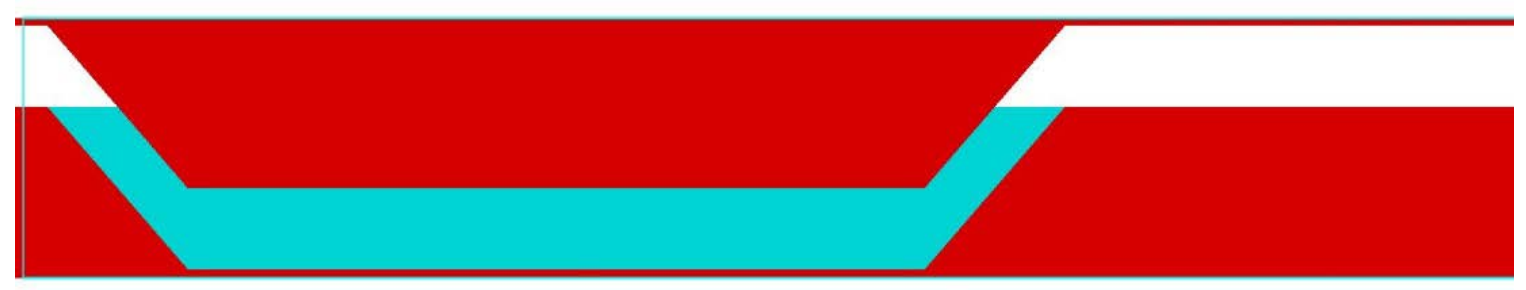

Figure 64: WD-35-2-WG (side view).

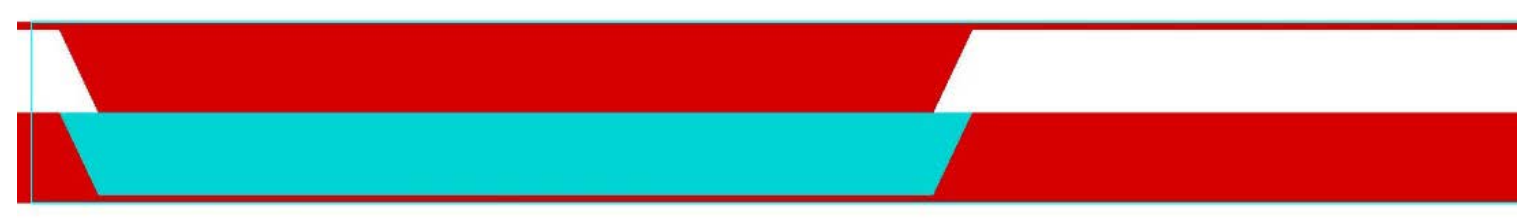

Figure 65: WD-55-1-WG (side view).

After observing the wave profiles from the simulation results in FLOW-3D, the 45 degrees inlet/outlet with a water depth of 1 feet produced the best uniform velocity and vertical-faced wave front. To make improvements in the wave profile, additional simulations were completed on Design 10 (WD-45-1-WG) with a thin layer of water placed downstream of the gate. The rationale was that the water layer would impede the lower portion of the wave front, helping to maintain the upper portion of the wave by decelerating the lower portion, thus, producing a near vertical wave better than the preliminary simulation. Three simulations were run including a thin film of water with depths of one cell, two cells, and three cells; the cell size of the FLOW-3D setup being $0.02 \mathrm{ft}$.

The sectional physical model of the WISD was designed in a 1:5 scale ratio assuming the Froude's number to be same for the model and the prototype. Similar to the numerical model, only one tube was designed out of total 10 tubes as each tube was defined to have exact same characteristics. Plexiglass was proposed as the material to construct the physical model to observe the behavior of the fluid. Using the physical model, possible discrepancies for both gate and motive force systems could be investigated, along with the flow behavior and the workability of mechanical components. Parameters for the sectional model using a 1:5 scale ratio are presented in Table 31. 
Table 31: Sectional Physical Model Parameters

\begin{tabular}{|l|l|l|}
\hline Model Scale Ratios and Prototype Equivalence \\
\hline Scale & Scale Value & Model to Prototype Equivalence \\
\hline Length scale & $\mathrm{L}_{\mathrm{r}}=5$ & $1 \mathrm{ft}=5 \mathrm{ft}$. \\
\hline Time scale & $\mathrm{t}_{\mathrm{r}}=2.24$ & $1 \mathrm{~s}=2.24 \mathrm{~s}$. \\
\hline Velocity scale & $\mathrm{V}_{\mathrm{r}}=2.24$ & $1 \mathrm{ft} / \mathrm{s}=2.24 \mathrm{ft} / \mathrm{s}$. \\
\hline Pressure Scale & $\mathrm{P}_{\mathrm{r}}=5$ & $1 \mathrm{psig}=5 \mathrm{psig}$. \\
\hline Design Parameters (For small scaled Model) & $1: 5$ \\
\hline Scale factor & Froude Number \\
\hline Prototype-Model Similarity & $11.36 \mathrm{fps}$. \\
\hline Velocity & 125 psf. (0.87 psig.) \\
\hline Pressure & Plexiglass \\
\hline Materials to be used (Model) & Steel \\
\hline Outer Walls & \\
\hline Inner plates \& gates
\end{tabular}

The physical model was designed as a module with the ability to change the channel length as deemed necessary for different scenarios. The inclined entrance was the first component providing a bend at different angles; 25 degrees, 35 degrees, and 45 degrees totaling at three in numbers. Air pressure would be applied in the first component with the help of an air compressor. The second component lies between the inclined entrance and the third component. This component consists of a bottom-hinged gate and one foot of length to allow the fluid motion to develop. The velocities would be measured at selected locations after the gate. The third component is a cuboidal box where the wavefront would be observed and the velocities would also be measured. The additional length segments that could alter the length of the reservoir and the development length was named as component four. Component five was the flange attached to other components for connecting/disconnecting capabilities. Table 32 is a list of components for the physical model and Figure 66 depicts the general layout of the sectional model for a 45 degrees inlet angle.

Table 32: Components of the Physical Sectional Model

\begin{tabular}{|l|l|l|l|}
\hline Component No. & Description & Quantity & Drawing No. \\
\hline \multirow{5}{*}{} & Inclined Inlet & & \multirow{3}{*}{$2-10$} \\
\cline { 2 - 3 } & $25^{\circ}$ & 1 & \\
\cline { 2 - 3 } & $35^{\circ}$ & 1 & $11-13$ \\
\cline { 2 - 3 } & $45^{\circ}$ & 1 & $14-17$ \\
\hline 2 & Gate Channel & 1 & \multirow{2}{*}{$18-26$} \\
\hline 3 & Outlet box & 1 & \\
\hline 4 & Additional Length component & 2 & \\
\cline { 2 - 3 } & $0.2^{\prime}$ & 4 & $27-28$ \\
\cline { 2 - 3 } & $0.4^{\prime}$ & 2 & 22 \\
\cline { 2 - 3 } & $1^{\prime}$ & 22 & \\
\hline 5 & Flanges & \multicolumn{2}{|l}{} \\
\hline
\end{tabular}




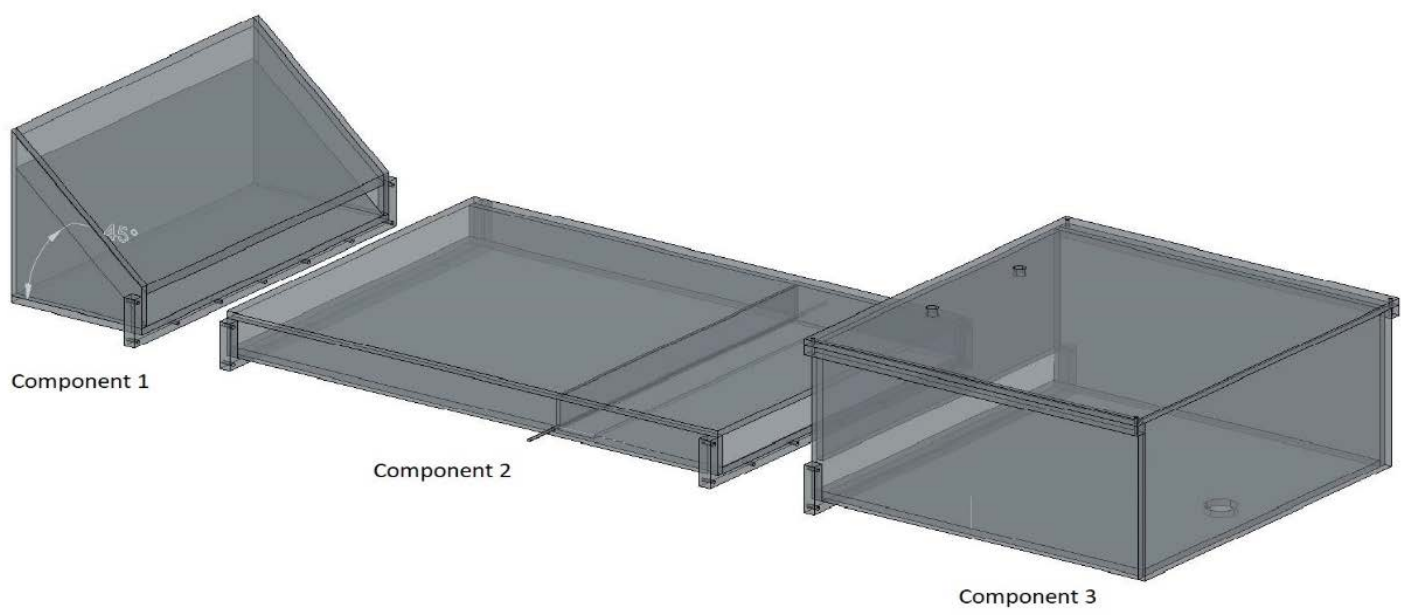

Figure 66: General layout of the sectional physical model

Component 2 is two feet wide, 0.2 feet high and three feet long with a gate located one foot from the exit. As mentioned earlier, using component 4 the lengths upstream and downstream could be increased. Note that only the length, entrance angle, and the motive air pressure were allowed to change for variation in data collection. Velocity sensors would be strategically placed in longitudinal and lateral directions to capture velocities at selected locations. Highly precise and accurate instruments would be used to record the air pressure in at least every $100^{\text {th }}$ of a second, the total simulation time being 1.5 seconds. A high-speed camera would also be used to observe the wave front downstream of the gate.

Another small-scale physical experiment study was proposed to verify the usability of air pressure and velocity measurement devices on a small-scale physical model before it was applied to a larger model. This allowed identification of potential drawbacks on the mechanism of the air pressure system and hence, make possible improvements for larger models. The possibility of using U-tubes in lieu of the gates could also be verified via this physical experiment study. The proposed conduit was a schedule 40 clear PVC pipe with a diameter of four inches. Using a clear PVC pipe, the fluid behavior could be observed upon application of the air pressure. Whereas, common PVC fittings were used for the elbows as clear PVC elbows were relatively expensive. Also, fluid behavior in the elbows was not a topic of interest for this study. The inlet and outlet of this arrangement were inclined at 45 degrees with a water depth of one foot. The wave developed downstream of the channel, and, velocity sensors were used to capture high velocities. The components were put together using PVC primer and glue for intact connections and prevention of leaks. A layout of the apparatus, with all components connected, is shown in Figure 67. 


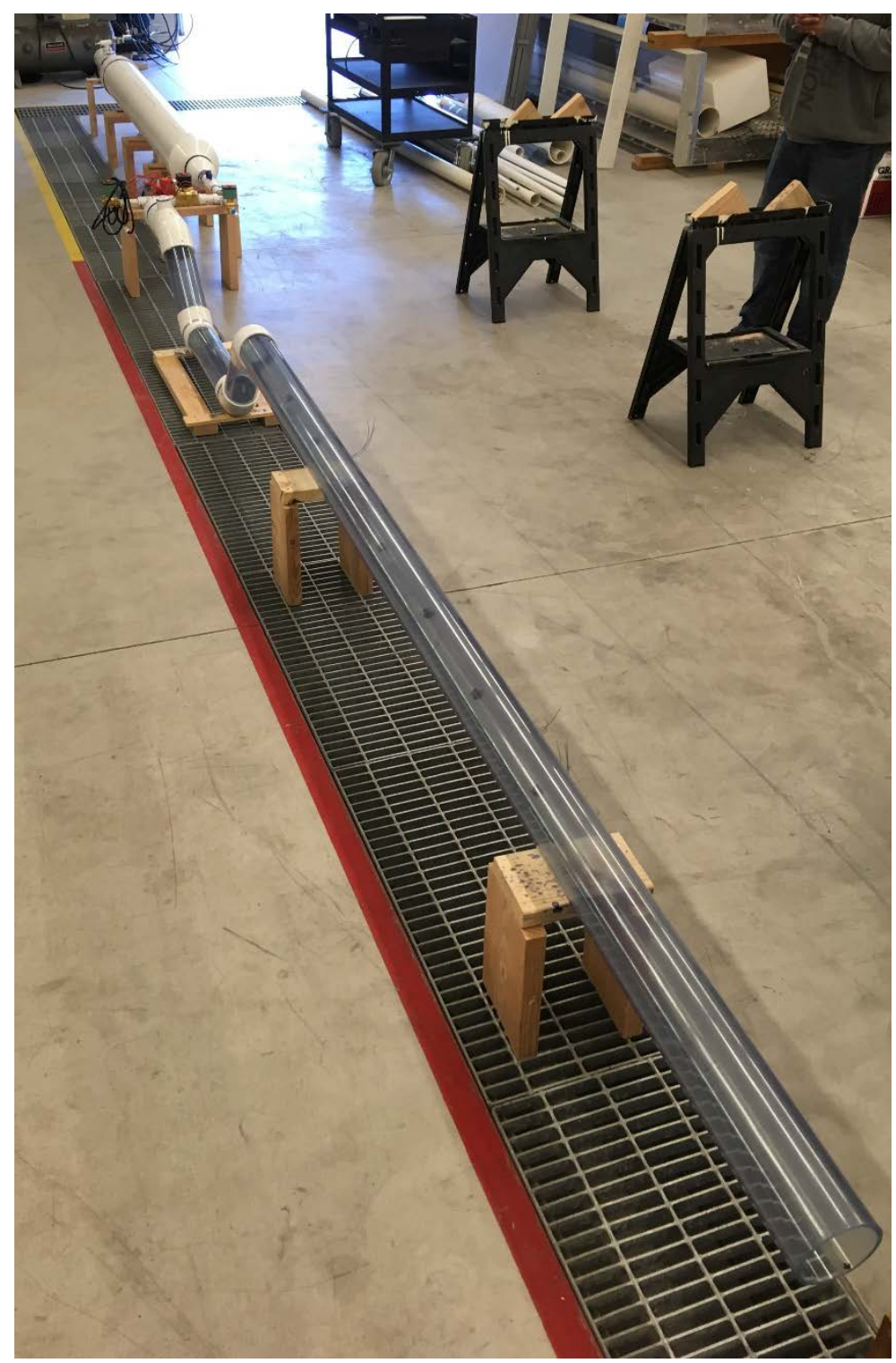

Figure 67: Layout of the physical experiment study.

The apparatus consisted of three components: 1) air pressure chamber, 2) solenoid valves and, 3) U-tube. The air pressure chamber was constructed with a schedule 40 eight inch PVC pipe and was eight feet long. The main purpose of using a chamber instead of a large compressor was to supply a sufficient volume of air into the third component. Large compressor could be used to supply air pressure into the U-tube, but most compressors would have a quarter inch outlet hose that would choke the airflow and limit the supply of air downstream. The volume within component one was large to try and provide a constant volume of air downstream into the U-tube and the desired pipe size could be used in the outlet. Additional PVC Tee's were added on to this component for connecting pressure compressors to pressurize the chamber and a transducer to measure the pressure. A ball valve was installed downstream to stop the air flow while pressurizing the tank. Figure 68 is a snapshot of the air pressure chamber. The Tee's can be observed on the left-hand side of the picture. 


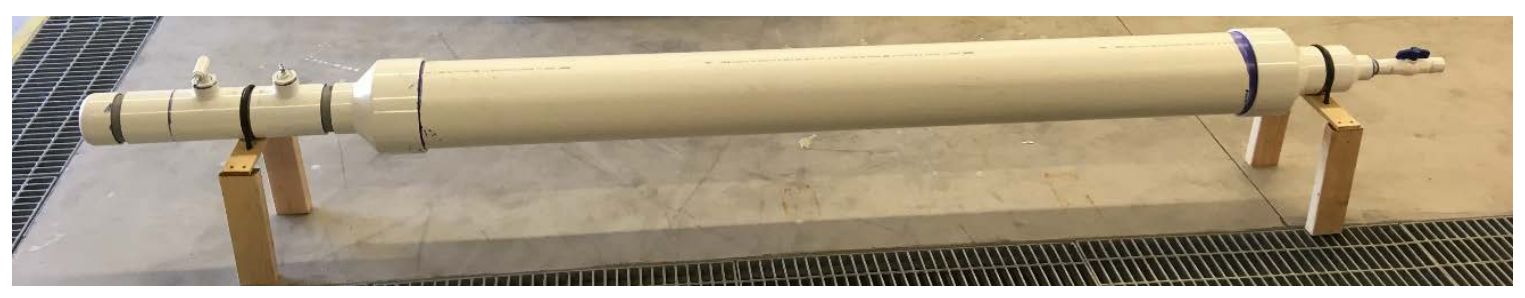

Figure 68: Component 1- Air pressure chamber.

Kinematic theories suggested that a pressure of 1.09 psig would need to be applied, then removed in 0.53 seconds to produce a steady 11.36 feet per second velocity wave. The valves controlling the pressure would, therefore, need to be fast-acting, actuated valves as manually opening and closing the valves could not be accomplished in a sufficient timeframe. Solenoid valves with response time less than 50 milliseconds were chosen for the task. The following diagrams illustrate the concept chosen by the team for applying the air pressure to the pipe model, (see Figure 69). Valves one and three are normally open (NO) solenoid valves that close when energized. Valve 2 is a normally closed (NC) solenoid valve that opens when energized. Power to all three valves is controlled by a multifunction timer relay. Figure 70 is a snapshot of the solenoid valves.

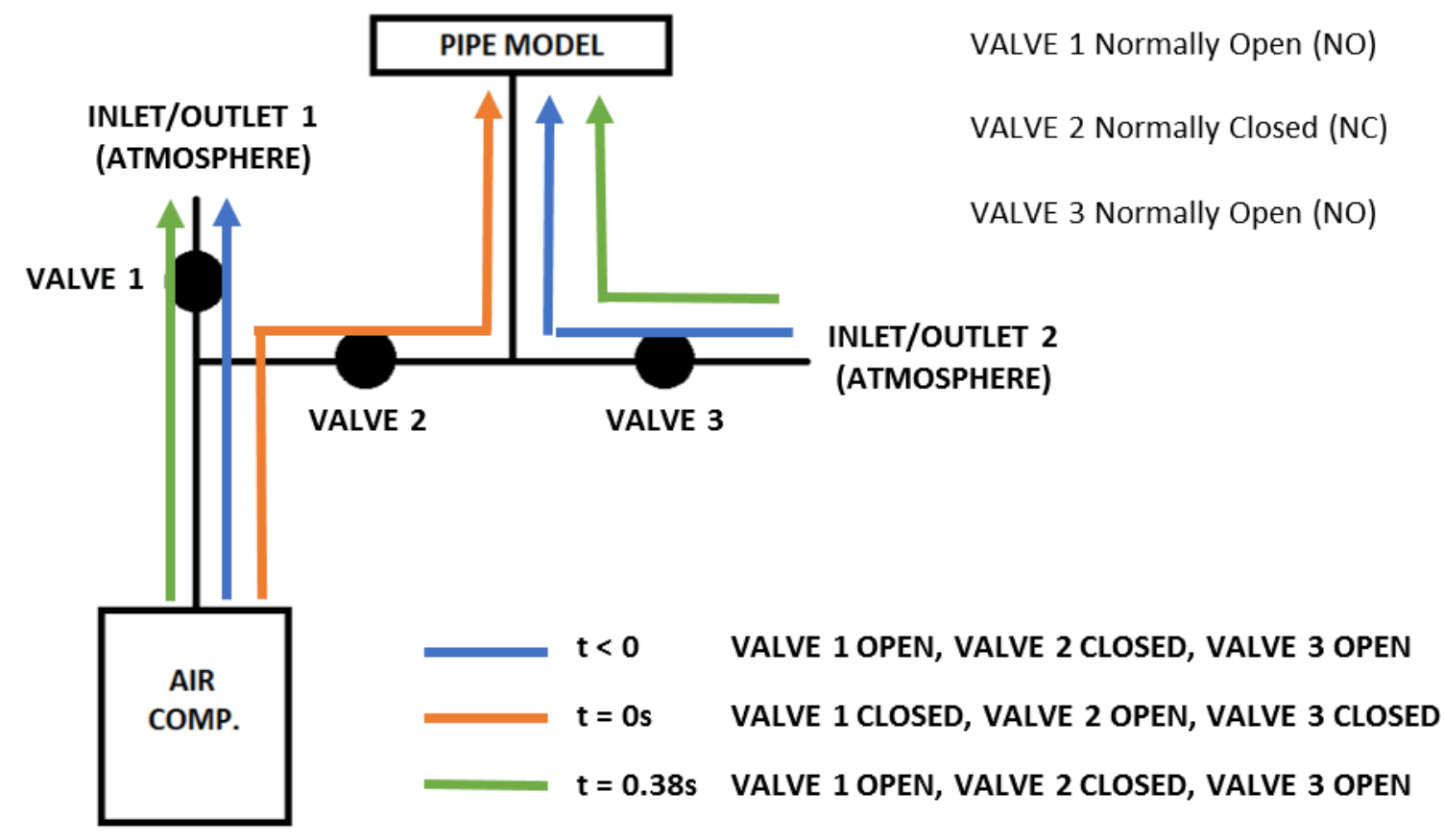

Figure 69: Diagram of pneumatic system for pipe model. 


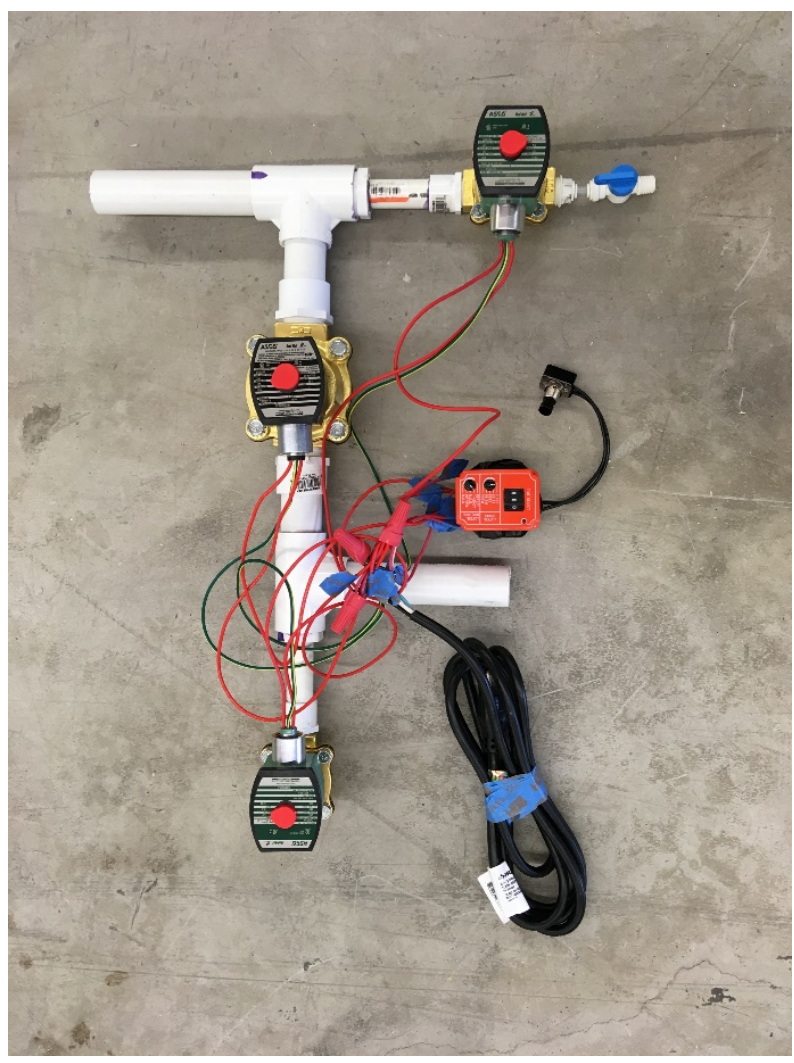

Figure 70: Solenoid valves arrangement

The U-tube was adopted in lieu of using gates in the system. Figure 71 is the side view drawing of the Utube with dimensions in feet (hatched area represents water). Figure 72 is a snapshot of the U-tube from the laboratory and the downstream end of this component was ten feet long. The wave profile developed in this downstream section and various sensors were inserted in selected locations to measure the velocity of the wave. Figure 73 shows pressure sensors located at every one foot along the length of the pipe for velocity measurement. At the upstream section of this component, a tee was added for measuring pressure.

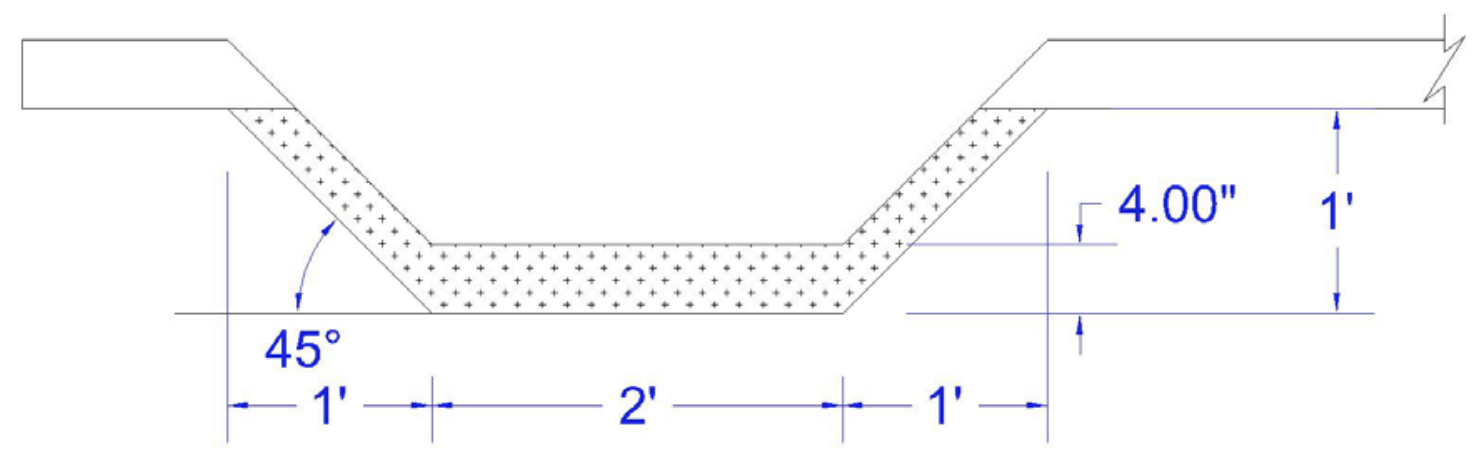

Figure 71: Physical experiment study U-tube. 


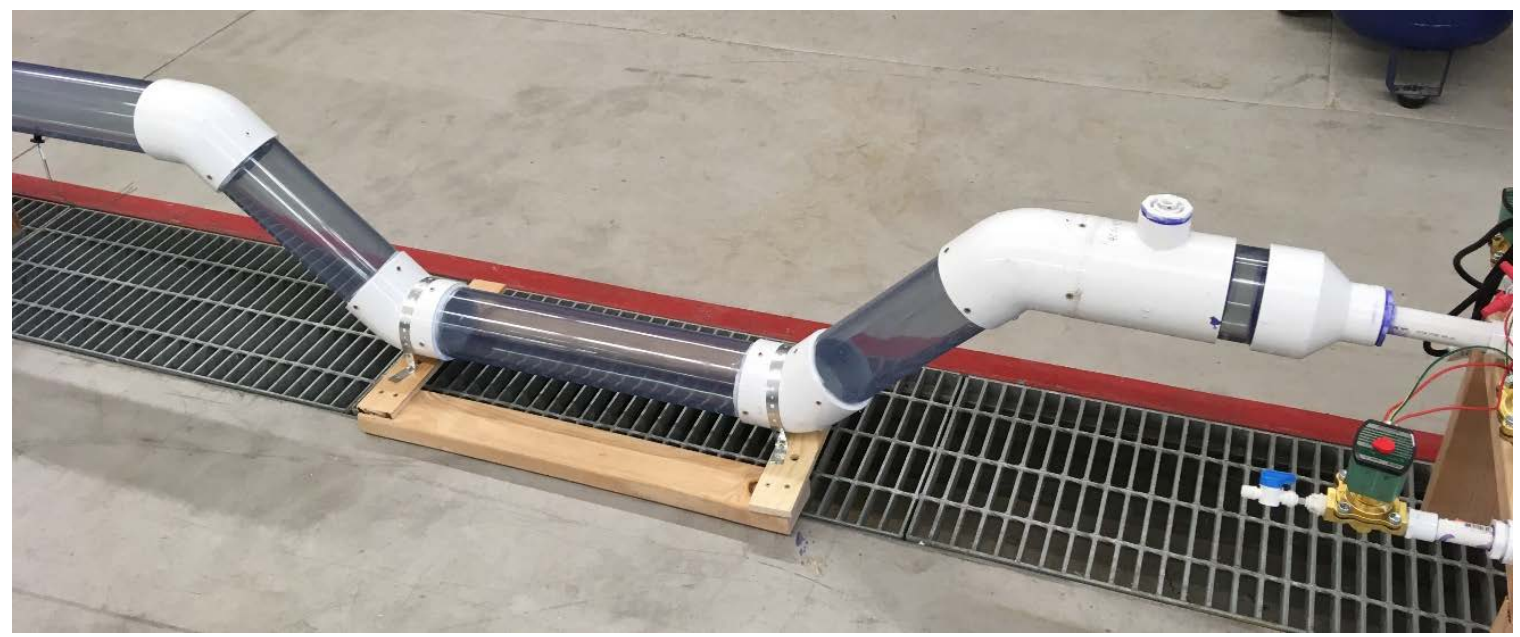

Figure 72: Component 3 (U-tube)

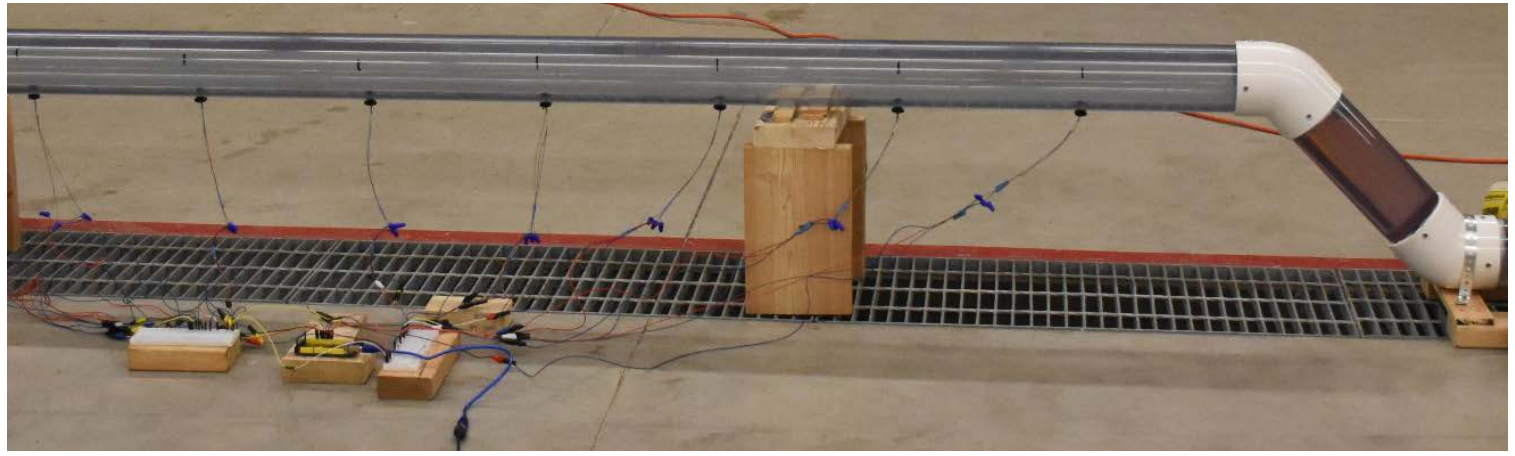

Figure 73: Velocity sensors

A multitude of models were simulated in FLOW-3D to determine an ideal design for the WISD capable of creating a steady wave with a near vertical wave section. FLOW-3D models with different inlet angles, along with different fluid depths are presented in chronological order of development. For all models, the development length, the distance between the gate and the outlet was fixed to six feet, the length of the fluid chamber, from the gate to the start of the inclined inlet was set to 14 feet as shown in Figure 74. Also, a pressure of 625 pounds per square foot was used to displace the fluid.

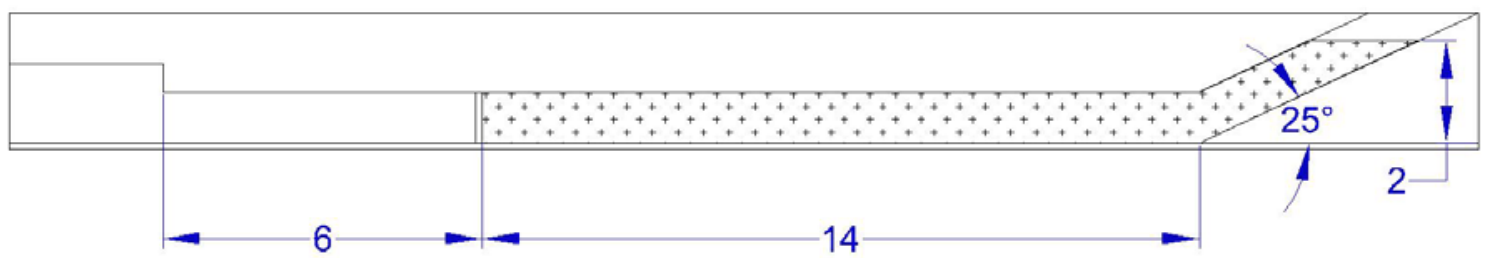

Figure 74: Design 8 (WD-25-2).

The different design configurations, labeled design $1-9$, are outlined in the following Figures and paragraphs. The configuration/model description is noted and specific comments related to the performance of the design are noted. 
Design 1 (WD-45-1)

Model Description: 45 degrees inlet angle with a fluid depth of one foot (see Figure 75).

Comments: $\quad$ The fluid accelerated quickly at $\mathrm{t}=0.7$ seconds up to 0.8 seconds but later continued steadily until $t=1.1$ seconds. The highest velocity recorded was 29.5 feet per second. The velocity of the fluid generated by this model was unsteady and the yielded wave profile was unsatisfactory (see Figure 76, color scale represents units in feet per second).

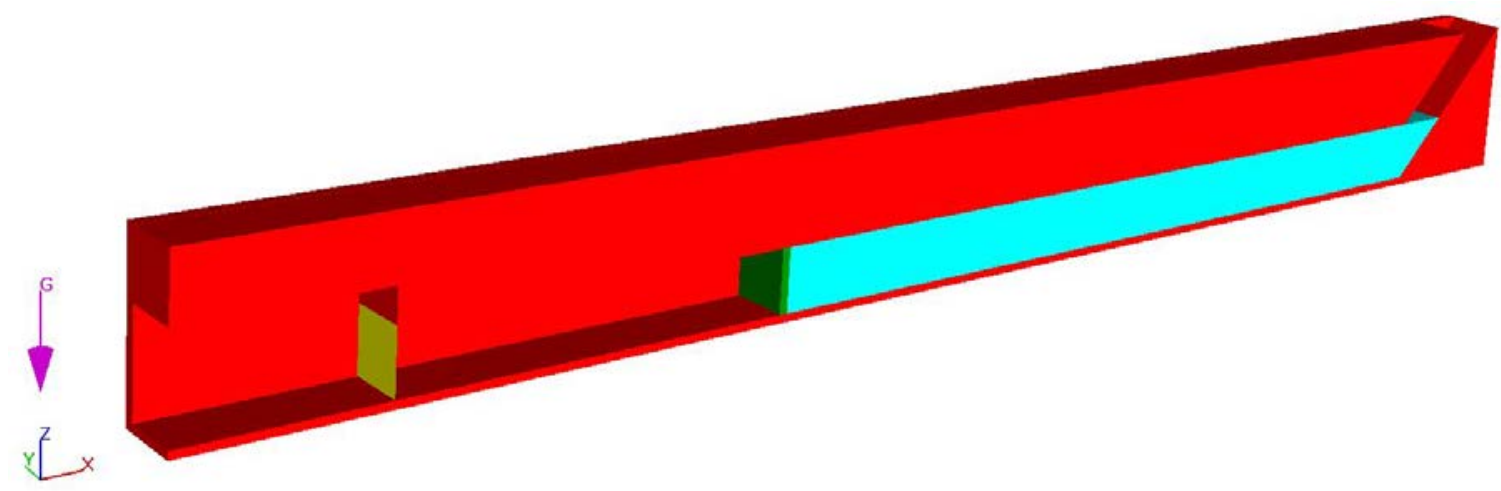

Figure 75: Design 1 (WD-45-1)

0.1

6.1

12.1

18.2

24.2

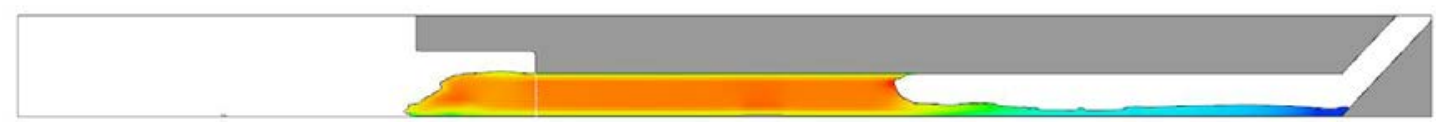

Figure 76: Design 1 (WD-45-1) - wave profile at $\mathrm{t}=0.8$ seconds

Design $2(W D-45-2)$

Model Description: 45 degrees inlet angle with a fluid depth of two feet (see Figure 77).

Comments:

The fluid accelerated rapidly at $\mathrm{t}=0.7$ seconds up to 0.8 seconds but later accelerated steadily until $t=1.2$ seconds. With a higher volume of water than Design 1 , it carries a higher momentum resulting in a peak velocity of 32 feet per second. The model is unsteady but produced an acceptable wave profile (see Figure 78, color scale represents units in feet per second). 


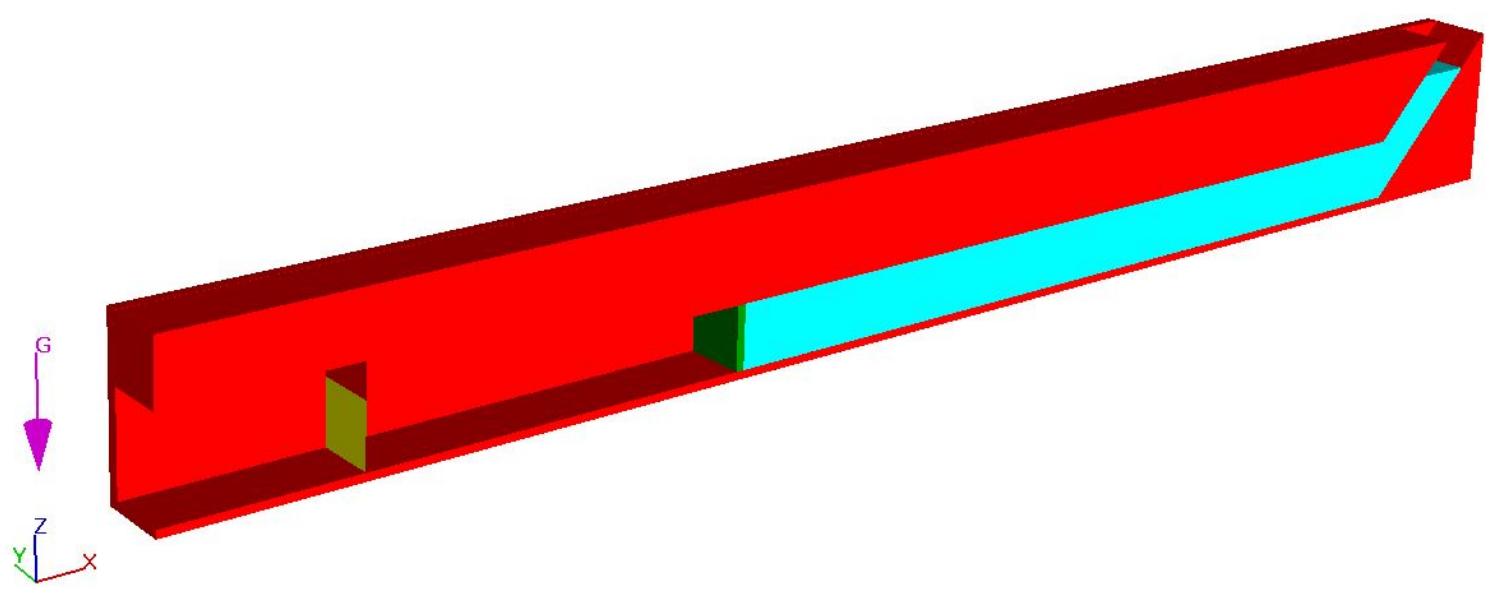

Figure 77: Design 2 (WD-45-2)

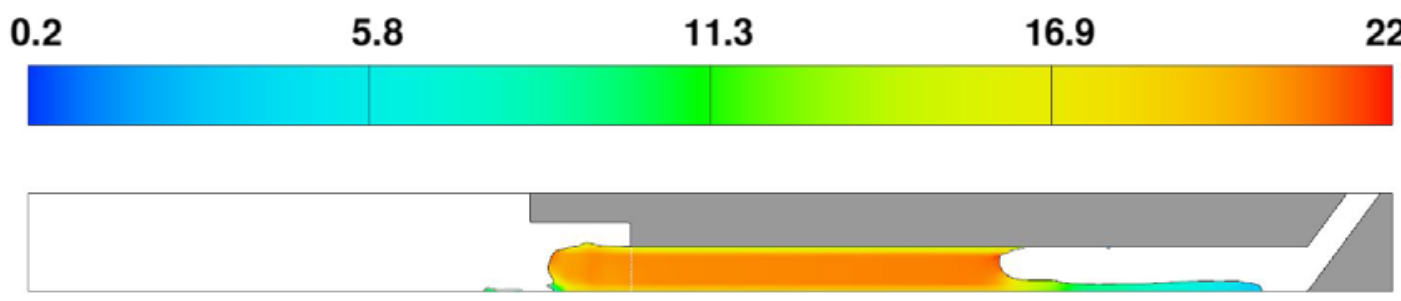

Figure 78: Design 2 (WD-45-2) - wave profile at $\mathrm{t}=0.8$ seconds

Design $3(W D-45-3)$

Model Description: 45 degrees inlet angle with a fluid depth of three feet (see Figure 79).

Comments:

At $\mathrm{t}=0.7$ seconds, water accelerated rapidly up to 0.9 seconds and later steadily accelerated until $\mathrm{t}=1.3$ seconds. With a higher volume of water than Design 1 and 2, its higher momentum resulted in a peak velocity of 36 feet per second. The results conclude the system to be unsteady but produced an acceptable wave profile (see Figure 80, color scale represents units in feet per second).

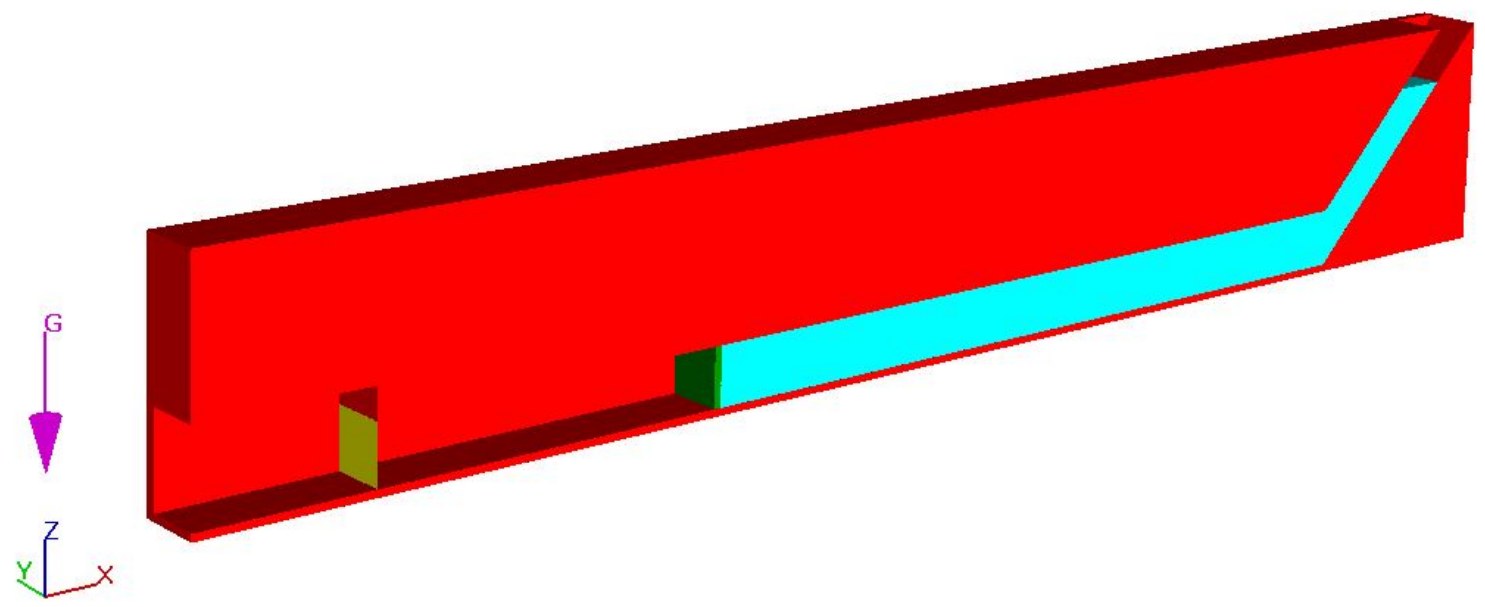

Figure 79: Design 3 (WD-45-3) 


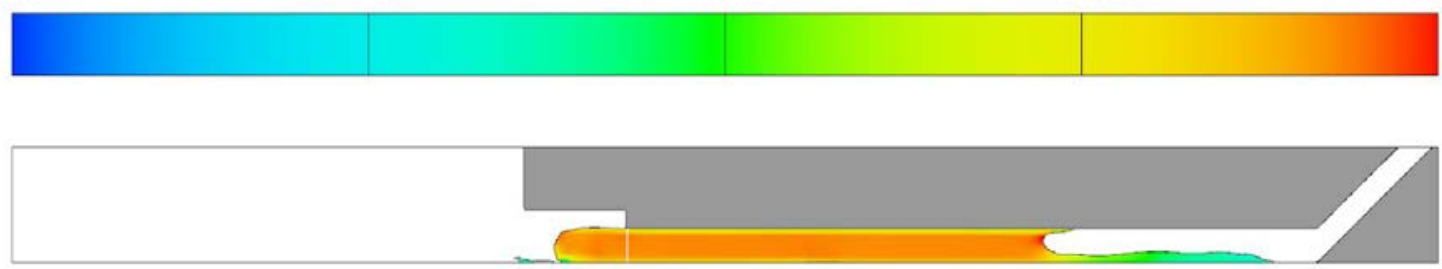

Figure 80: Design 3 (WD-45-3) - wave profile at $\mathrm{t}=0.8$ seconds

Design 4 (WD-35-1)

Model Description: 35 degrees inlet angle with a fluid depth of one foot (see Figure 81).

Comments: $\quad$ At $\mathrm{t}=0.7$ seconds, water accelerated rapidly up to 0.8 seconds and later steadily accelerated until $\mathrm{t}=1.1$ seconds. This model resulted in a peak velocity of 38.6 feet per second. This model has a relatively higher cross-sectional area for the initial air pressure than the 45 degrees model, which would produce a higher motive force resulting in a larger peak velocity. The results show the system to be unsteady with a rather undesirable wave profile (see Figure 82, color scale represents units in feet per second).

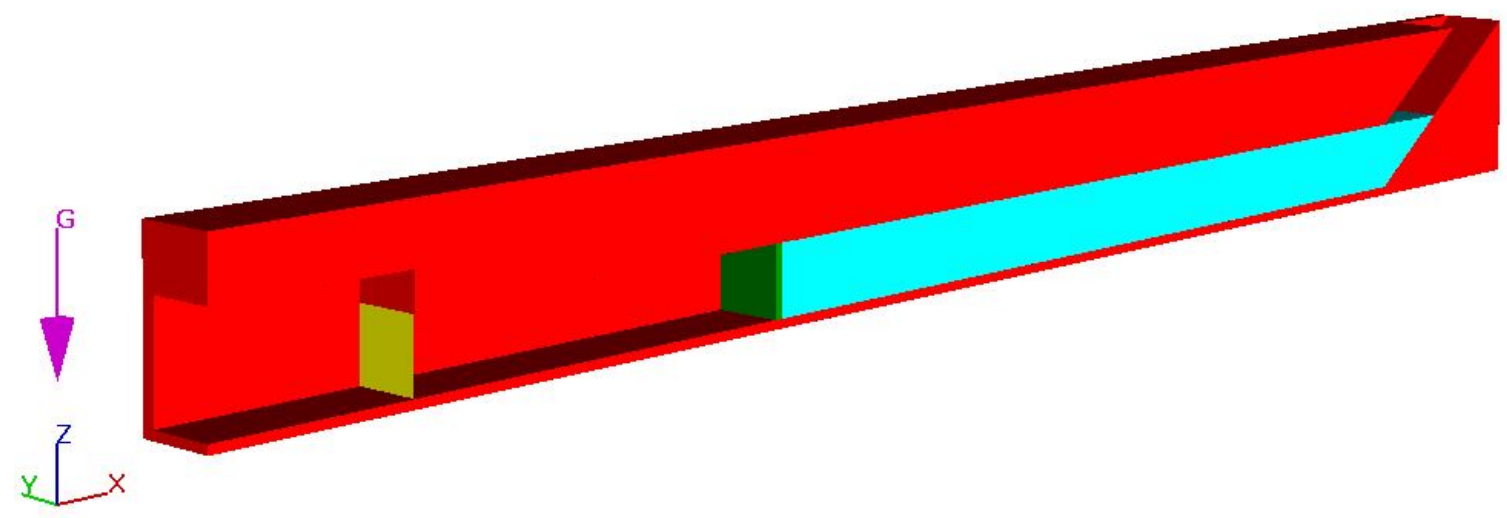

Figure 81: Design 4 (WD-35-1)

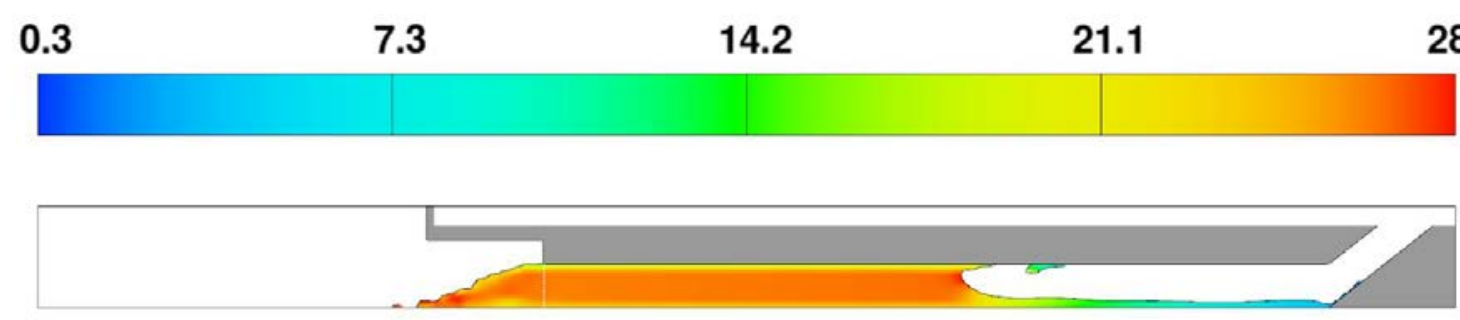

Figure 82: Design 4 (WD-35-1) - wave profile at $\mathrm{t}=0.4$ seconds

Design 5 (WD-35-2)

Model Description: 35 degrees inlet angle with a fluid depth of two feet (see Figure 83).

Comments:

At $\mathrm{t}=0.7$ seconds, water accelerated rapidly up to 0.8 seconds and later steadily accelerated until $t=1.2$ seconds. This model resulted in a peak velocity of 34.2 feet per second. Unlike the 45 degrees model, the peak velocity is smaller than Design 4 (WD-35-1) that has a depth 
of $1 \mathrm{ft}$. The velocity is not constant over the time period and the wave profile does not meet the expectations of the study (see Figure 84, color scale represents units in feet per second).

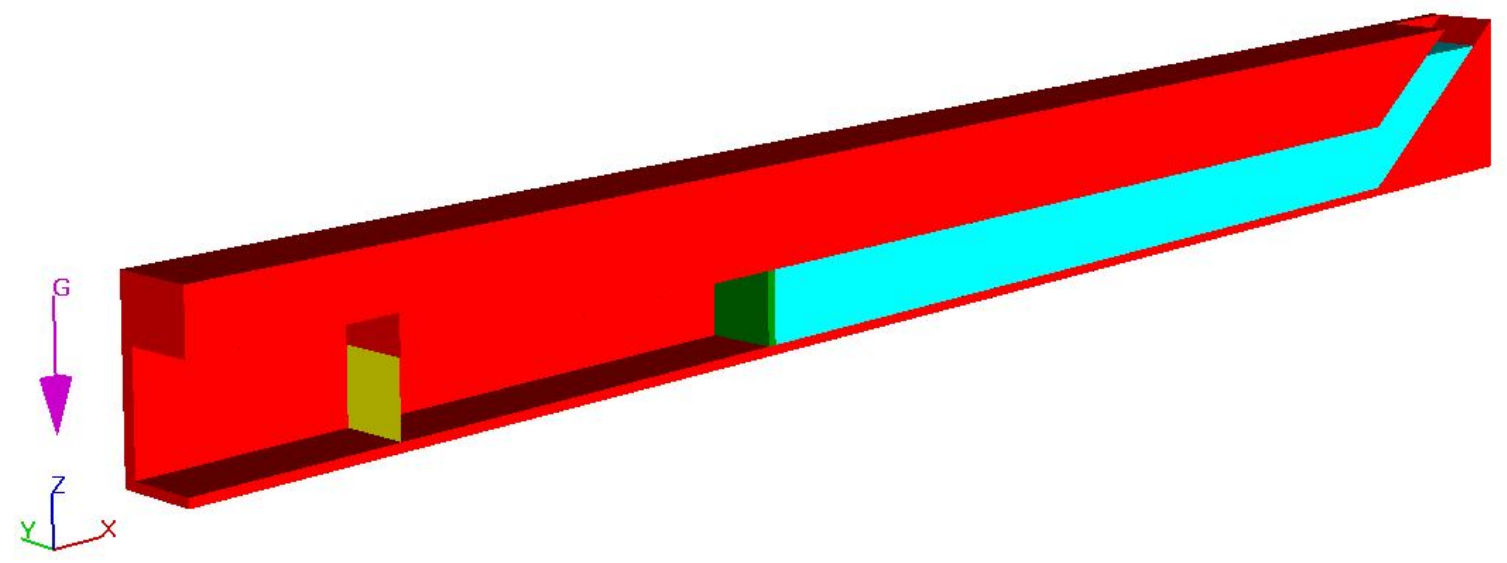

Figure 83: Design 5 (WD-35-2)
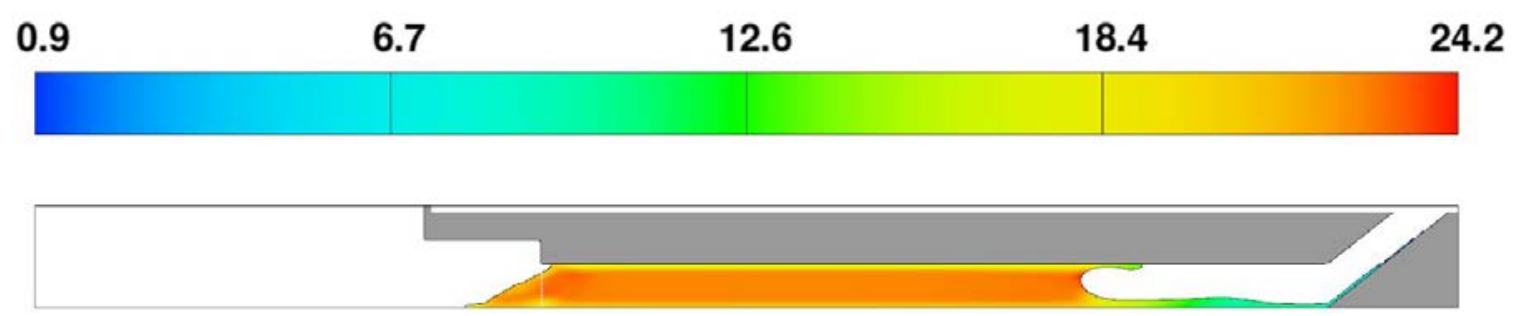

Figure 84: Design 5 (WD-35-2) - wave profile at $t=0.4$ seconds

Design 6 (WD-35-3)

Model Description: 35 degrees inlet angle with a fluid depth of three feet (see Figure 85).

Comments: $\quad$ At $\mathrm{t}=0.7$ seconds, water accelerated rapidly up to 0.9 seconds and later steadily accelerated until $t=1.3$ seconds. This model resulted in a peak velocity of 33.7 feet per second. The velocity is not constant over a time period and the wave profile does not meet the expectations of the study (see Figure 86, color scale represents units in feet per second).

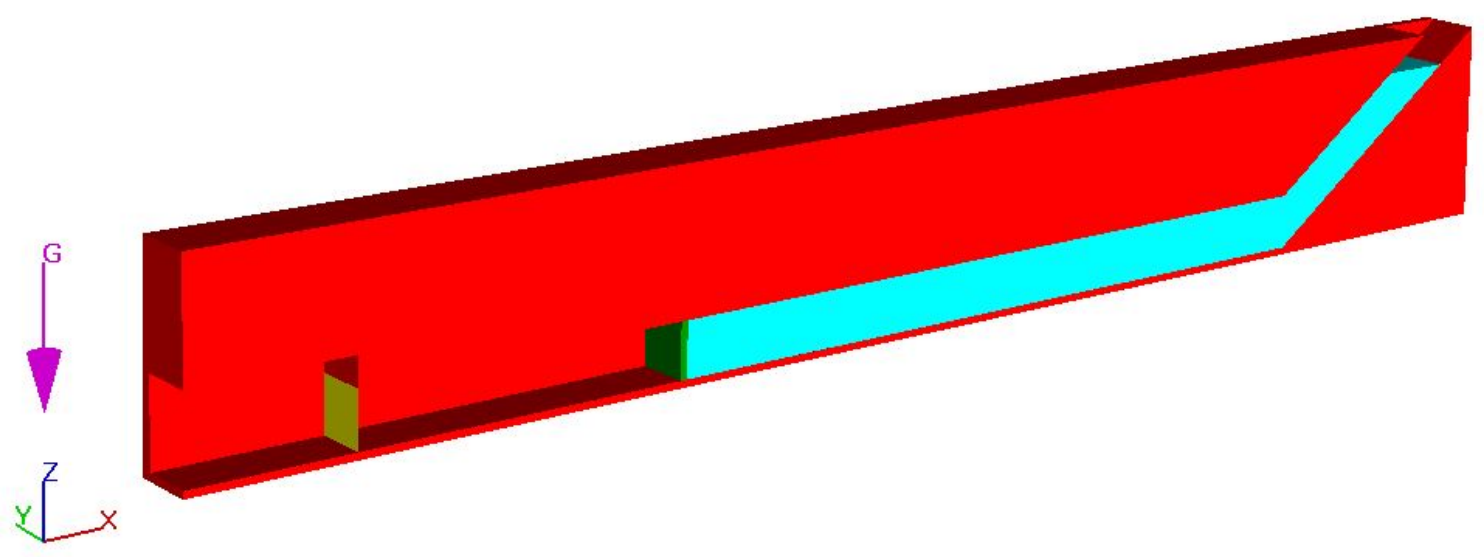

Figure 85: Design 6 (WD-35-3) 


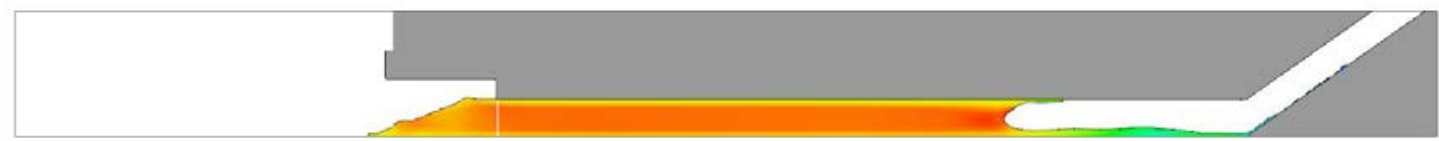

Figure 86: Design 6 (WD-35-3) - wave profile at $\mathrm{t}=0.6$ seconds

Design 7 (WD-25-1)

Model Description: 25 degrees inlet angle with a fluid depth of one foot (see Figure 87).

Comments:

Water accelerated rapidly at $\mathrm{t}=0.7$ seconds up to 0.8 seconds and later steadily accelerated until $\mathrm{t}=1.1$ seconds. This model resulted in a peak velocity of 33 feet per second. Unexpectedly, this model did not follow the trend set by previous simulations; the increased surface area for the initial pressure of this simulation compared to 25 and 35 degrees inlet should have produced a higher peak velocity due to the increased force. However, the peak velocity for this model is lesser than the 35 degrees geometry with one foot of depth. The velocity is unsteady and the wave profile does not meet the expectations of the study (see Figure 88, color scale represents units in feet per second). Unusually, the top portion of the wave is ahead of the bottom portion, unlike previous model results.

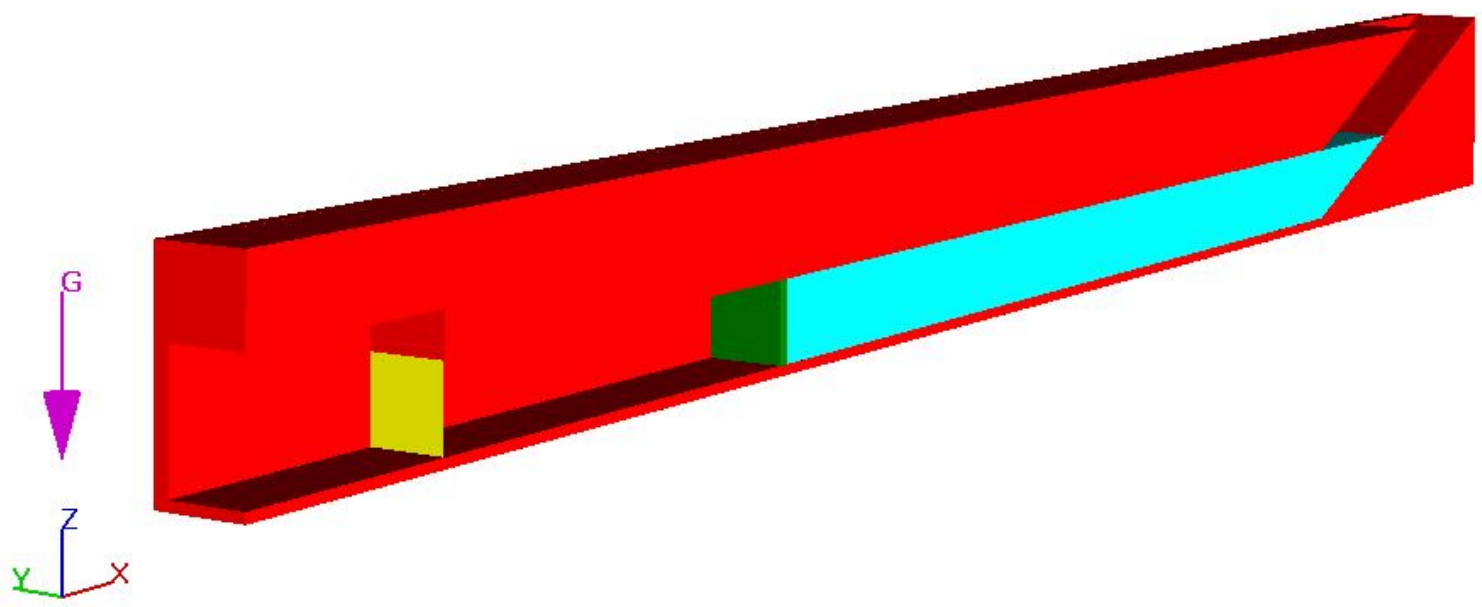

Figure 87: Design 7 (WD-25-1)

\section{4}

6.3

12.2

18.1

24.0

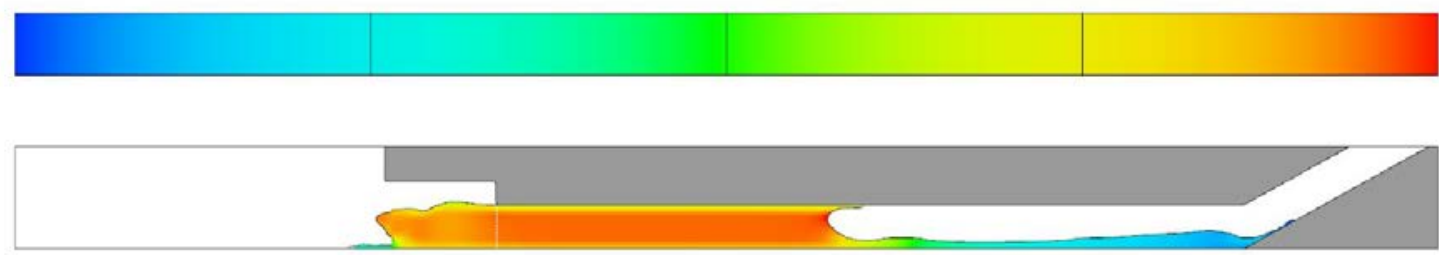

Figure 88: Design 7 (WD-25-1) - wave profile at $\mathrm{t}=0.8$ seconds

Design $8(W D-25-2)$

Model Description: 25 degrees inlet angle with a fluid depth of two feet (see Figure 89). 
Comments: $\quad$ Water accelerated rapidly at $\mathrm{t}=0.7$ seconds up to 0.8 seconds, followed by a rather steady acceleration until $t=1.3$ seconds. This model resulted in a peak velocity of 38.4 feet per second. The velocity is unsteady and the observed wave profile is acceptable (see Figure 90 , color scale represents units in feet per second).

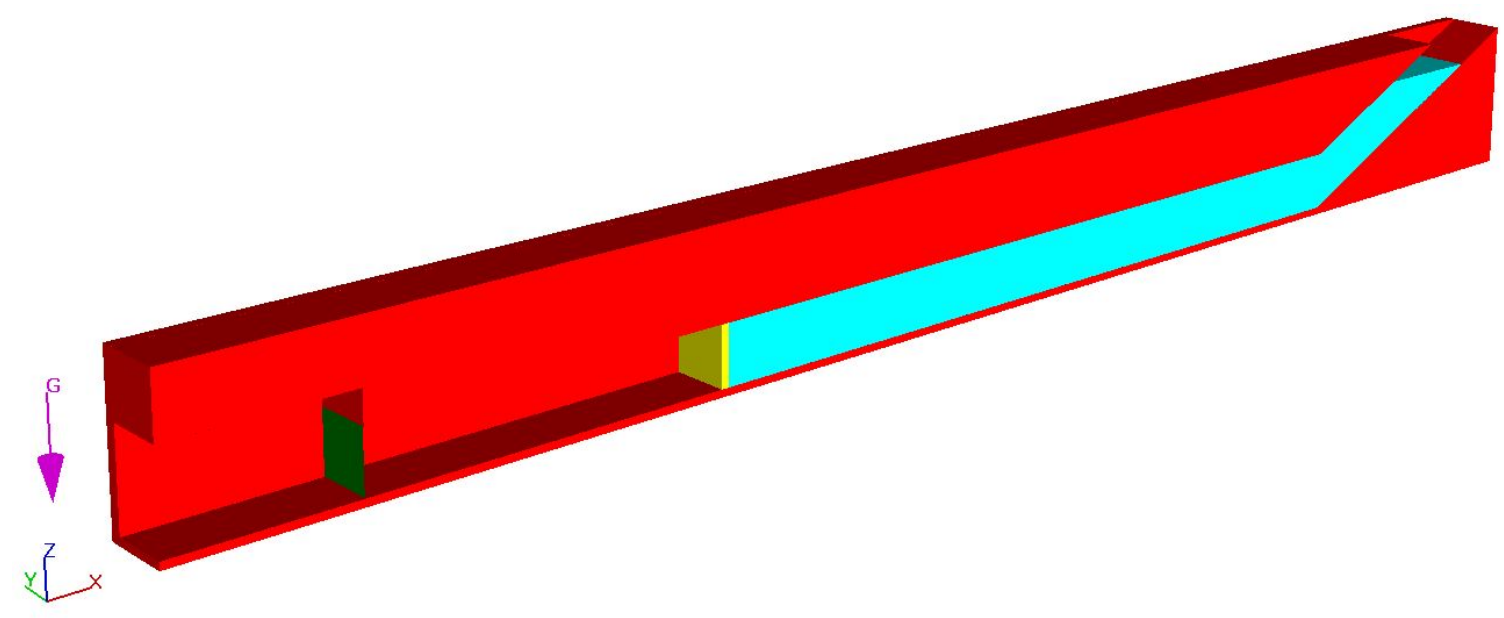

Figure 89: Design 8 (WD-25-2)
0.9
6.1
11.3
16.5
21.7

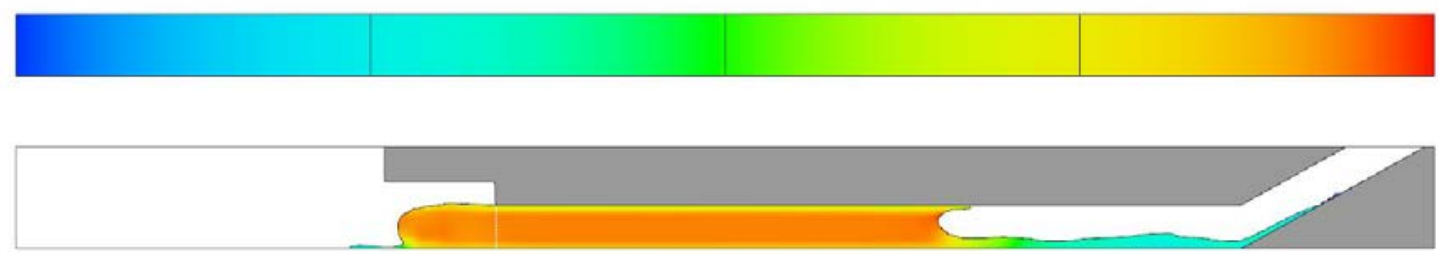

Figure 90: Design 8 (WD-25-2) - wave profile at $\mathrm{t}=0.85$ seconds

Design 9 (WD-25-3)

Model Description: 25 degrees inlet angle with a fluid depth of three feet (see Figure 91).

Comments:

The fluid accelerated steadily at $\mathrm{t}=0.7$ seconds up to 0.8 seconds, followed by a rapid acceleration until $t=1.4$ seconds. This model has the highest volume of water resulting in a peak velocity of 36.2 feet per second which is quite strange as it was expected this model would have the highest peak velocity due to the initial depth of three feet. The velocity is not constant but the wave profile is good enough (see Figure 92, color scale represents units in feet per second). 


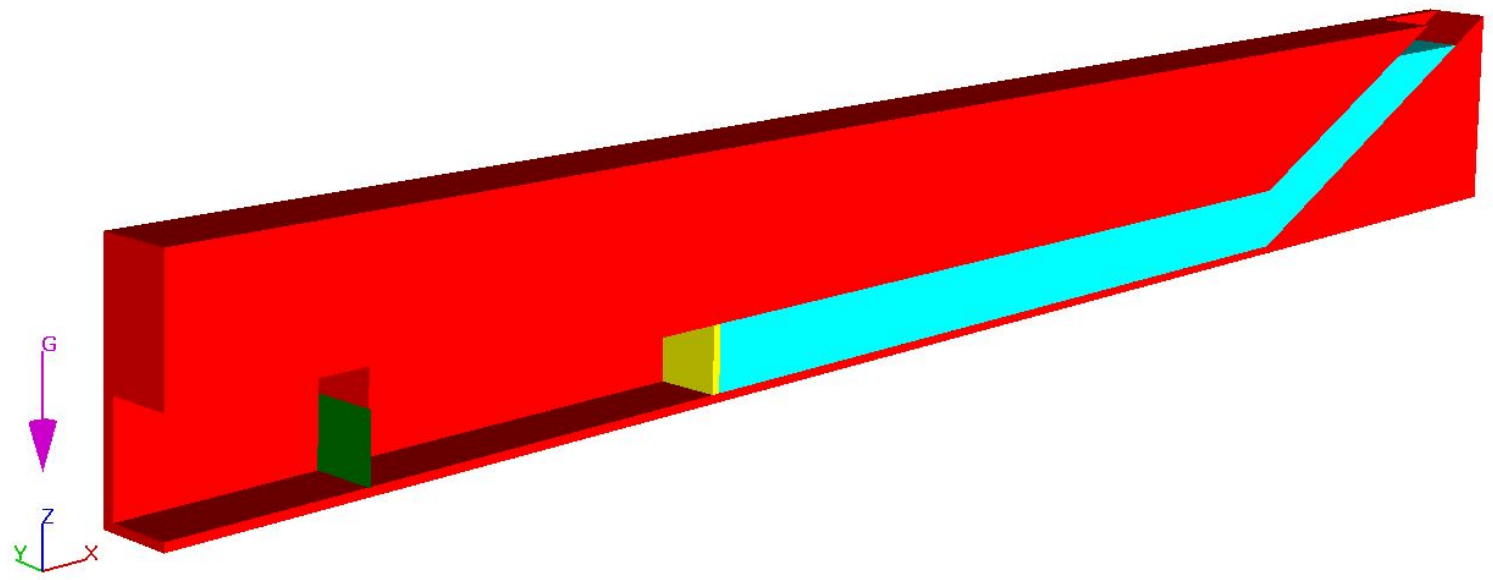

Figure 91: Design 9 (WD-25-3)

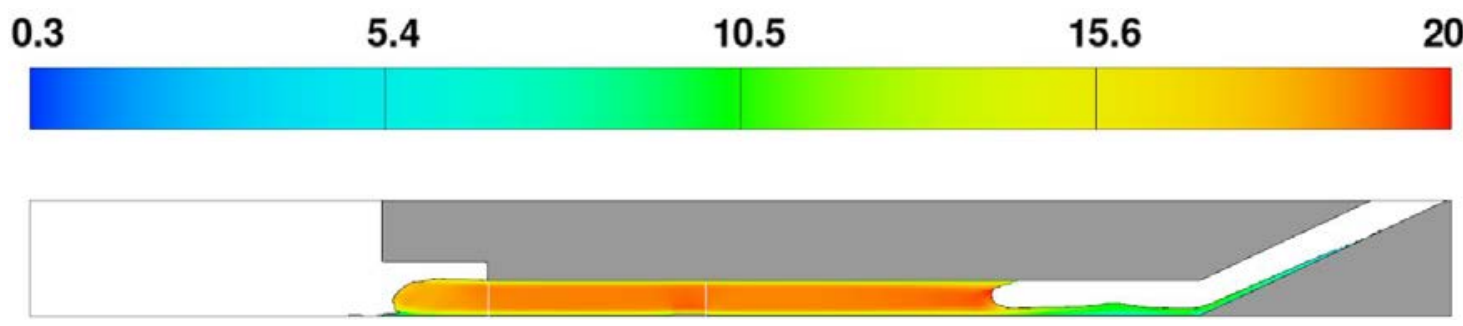

Figure 92: Design 9 (WD-25-3) - wave profile at $\mathrm{t}=0.9$ seconds

The simulation configurations included inlet angles at 25, 35, and 45 degrees with upstream depths ranging from one to three feet. All the simulations had similar behavior (see Figure 93); the velocities would sharply increase at 0.7 seconds and then gradually increase for a range from 0.8 seconds to 1.3 seconds followed by a sudden decrease as the water exited the flow chamber, except for the setup with 25 degrees of inlet angle with a fluid depth of one foot, which would gradually increase from 0.7 seconds to 0.8 seconds followed by a steep increase up to 0.9 seconds. 


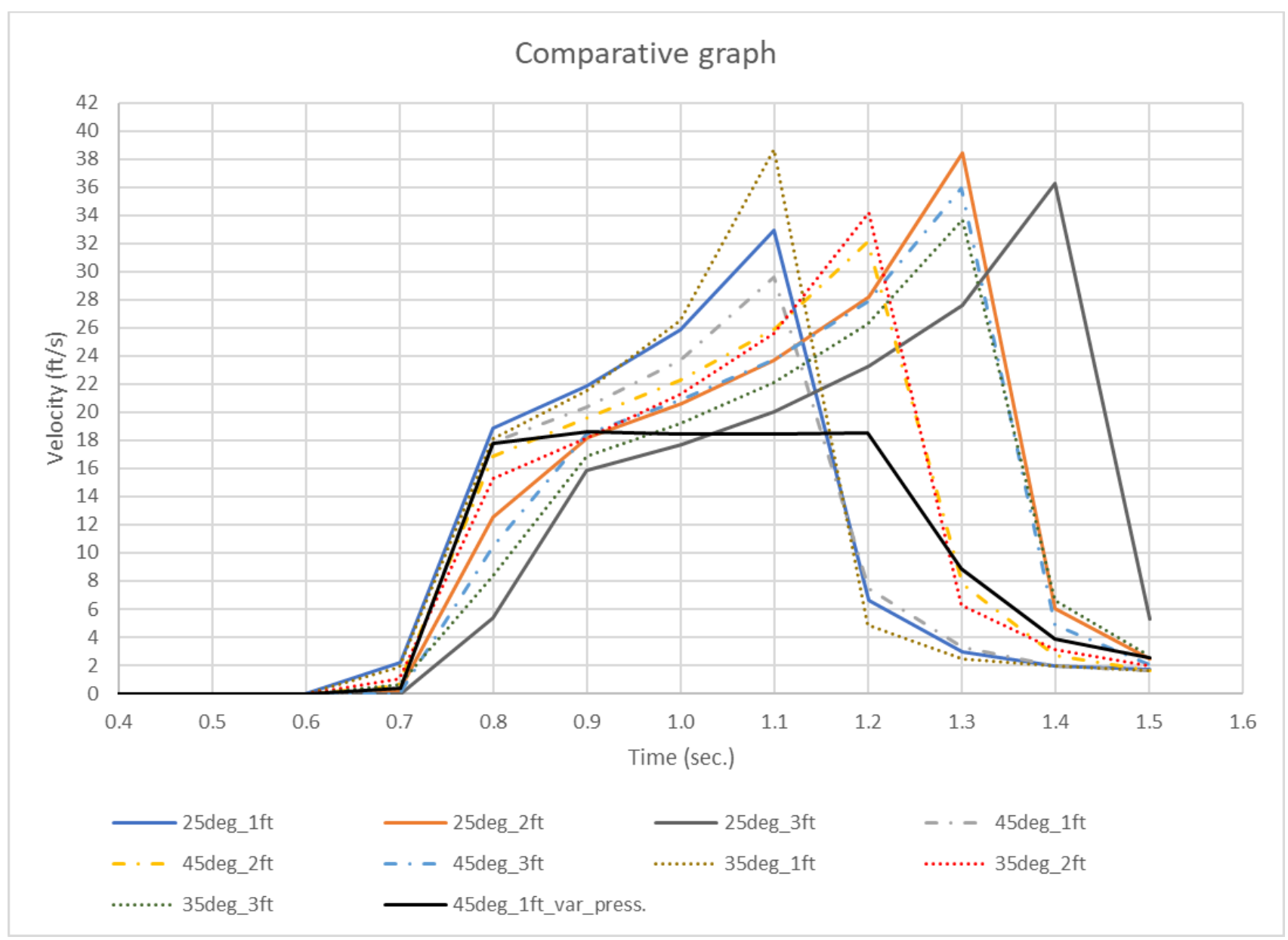

Figure 93: Velocity vs time

Comparing the one-foot depth inlet for angles 25, 35, and 45 degrees, the results show that the inlet angle does increase the motive force, moving the water through the chamber with a slightly higher initial response at 0.8 seconds and a larger peak at 1.1 seconds. This is expected since the initial pressure force is pressure multiplied by the area and the 25 degrees inlet provides more area. Additionally, increasing the inlet depth slows the initial response at 0.7 seconds but produces a higher peak that is delayed. The higher peak is attributed to the increase in the initial inlet depth and the time delay is created by the change in inertia required to move the additional mass.

Initially, a trial and error process was used to select the air pressure that was applied to the static water to obtain a steady state velocity. To help understand this process better, analytical kinematic theories outlined in literature were adapted to compute the ideal variation in the air pressure. Theoretically, the fluid should maintain a steady velocity if it maintains its initial geometry similar to a solid block. However, a real fluid does not follow this assumption, and thus, as expected, the numerical simulations using FLOW-3D has slight differences in its results compared to the theoretical values, when the variation in air pressure was applied to the system rather than a constant air pressure behind the system. The applied variation in air pressure allowed the fluid to maintain velocities ranging from 27.8 feet per second to 28.8 feet per second for a duration of 0.4 seconds instead of the expected velocity of 25.4 feet per second for the geometry with 25 degrees inlet and a fluid depth of 2 feet. Figure 94 shows a plot of velocity (feet per second) and time (seconds) for the geometry with a 25 degrees inlet angle and a fluid depth of 2 feet for the CFD simulation. These results satisfy the expectations of the WISD, with an approximately constant velocity from 0.8 seconds to 1.2 seconds. 


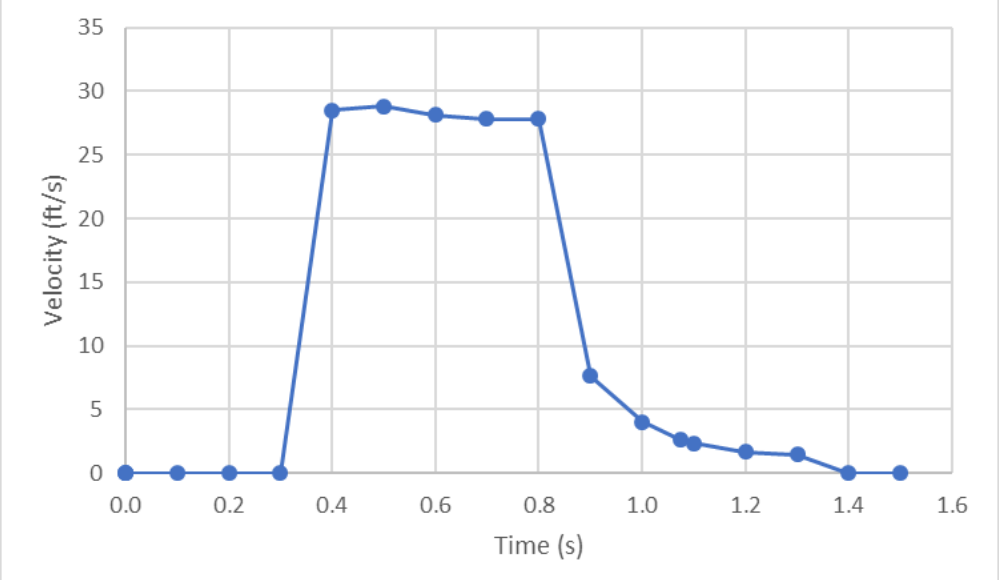

Figure 94: Velocity vs time (25 degrees inlet $-2 \mathrm{ft}$. depth)

The velocities were recorded via a baffle placed at the same location for all simulations. The results were plotted on a graph with velocity (feet per second) on the y-axis and time (seconds) on the x-axis as shown in Figure 95. It can be observed that most geometries with a depth of one foot have a near constant velocity of approximately 25 feet per second starting within $\mathrm{t}=0.4$ to 0.5 seconds that is conserved for almost $\mathrm{t}=1$ seconds. Whereas, the geometry with 55 degrees and the 25 degrees inlet-outlet have not produced acceptable results as shown in Figure 95 (c). The initial bump on the graphs is believed to appear from splashing and turbulent nature of water. 
(a)

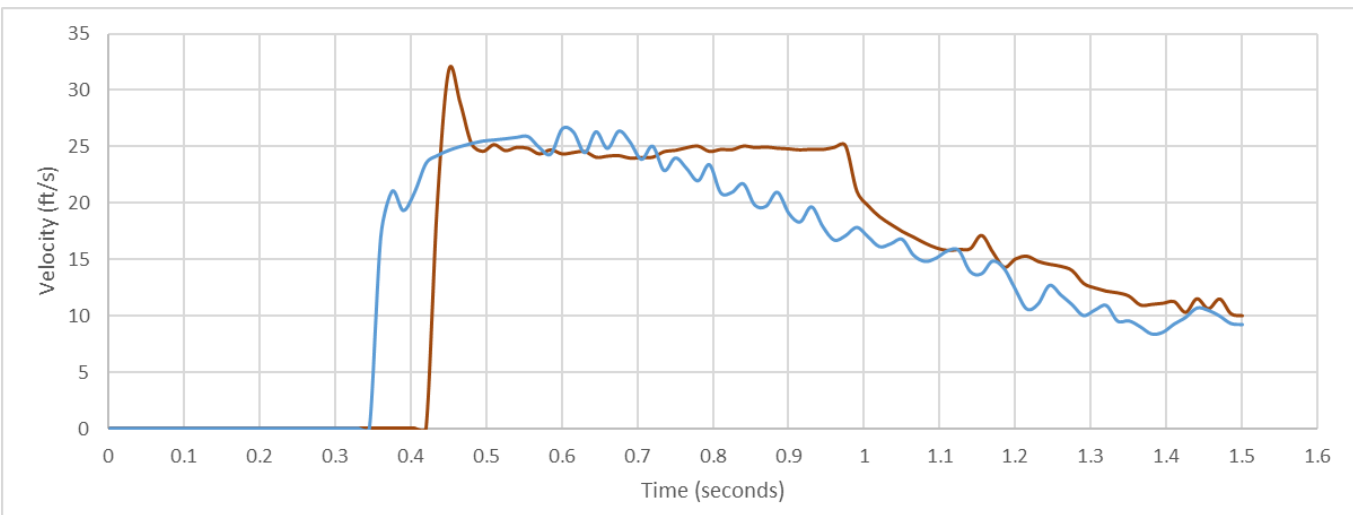

-WD-45-1-NG -WD-45-2-NG

(b)

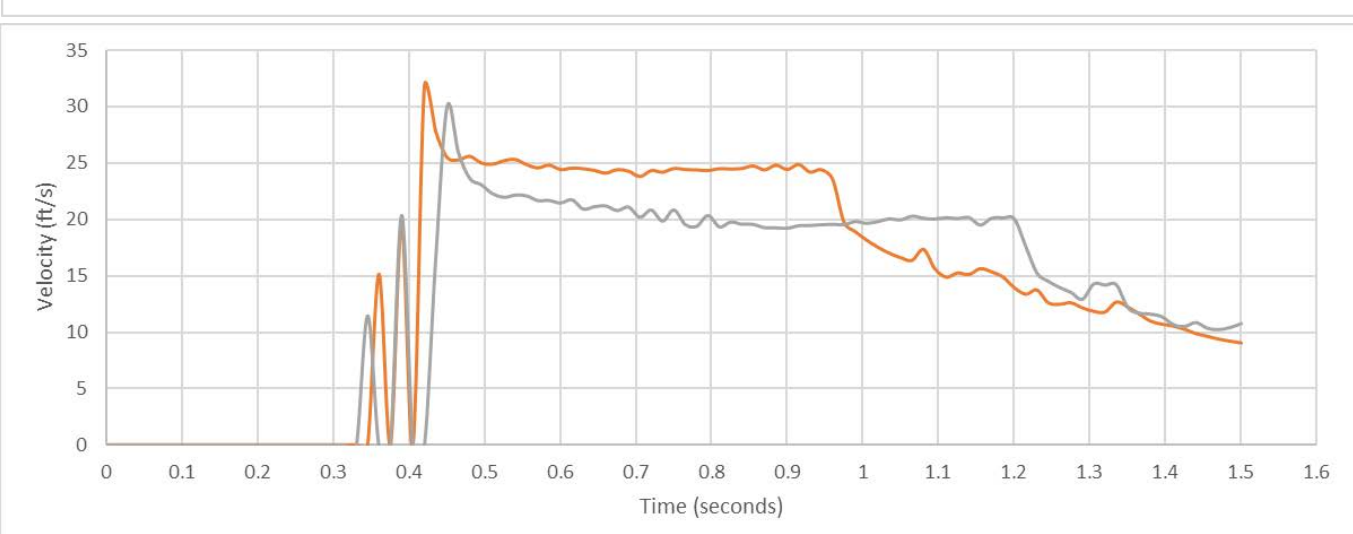

-WD-35-1-NG —WD-35-2-NG

(c)

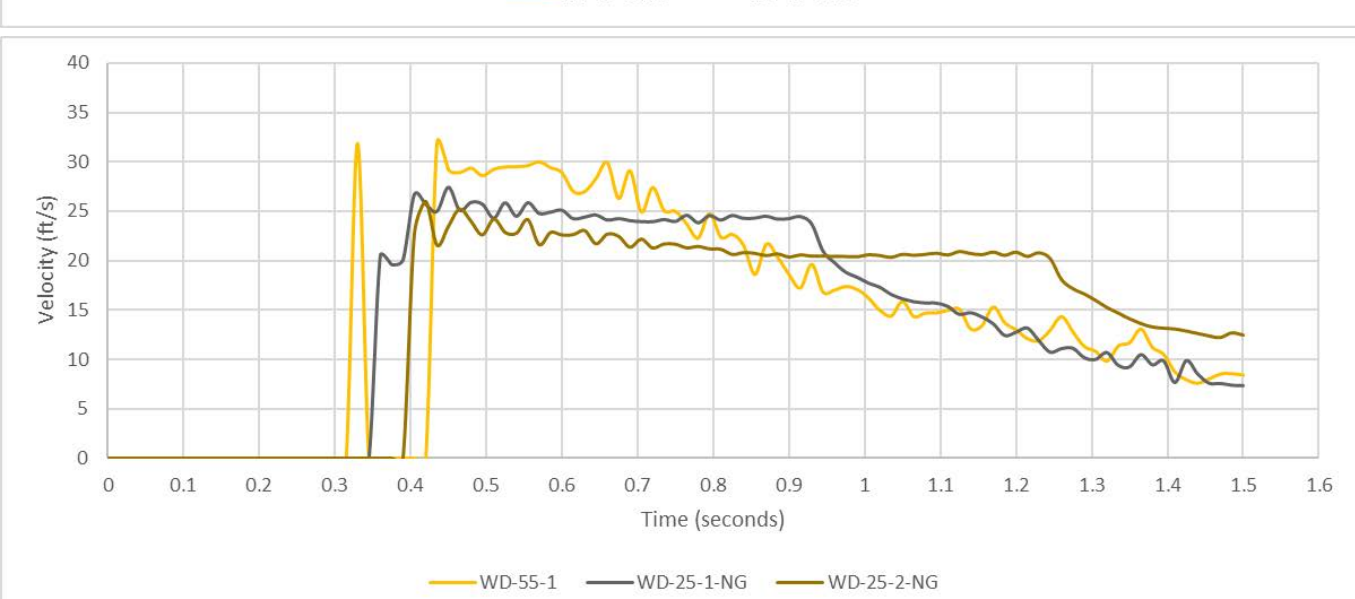

Figure 95: Velocity - vs - time (without gates) for geometries with: (a) 45 degrees; (b) 35 degrees; (c) 55 degrees and 25 degrees

Primarily, three parameters were focused in the physical experimental test: 1) U-tube pressure, 2) wave velocity and, 3) wave profile. The U-tube pressures and wave velocities were adjusted by controlling the chamber pressure (listed in Table 33). The wave profiles generated by all test configurations were near vertical, which was one of the major objectives of this study. Among a number of test configurations, a chamber pressure of 2.75 psig yielded an almost constant velocity and a U-tube pressure of 1.1 psig. The target velocity was 11.3 feet per second and the adopted kinematics suggested a U-tube pressure of 1.09 psig would be required to displace the water in 
motion for the mentioned velocity. The wave profile produced by this configuration was acceptable (see Figure 96).

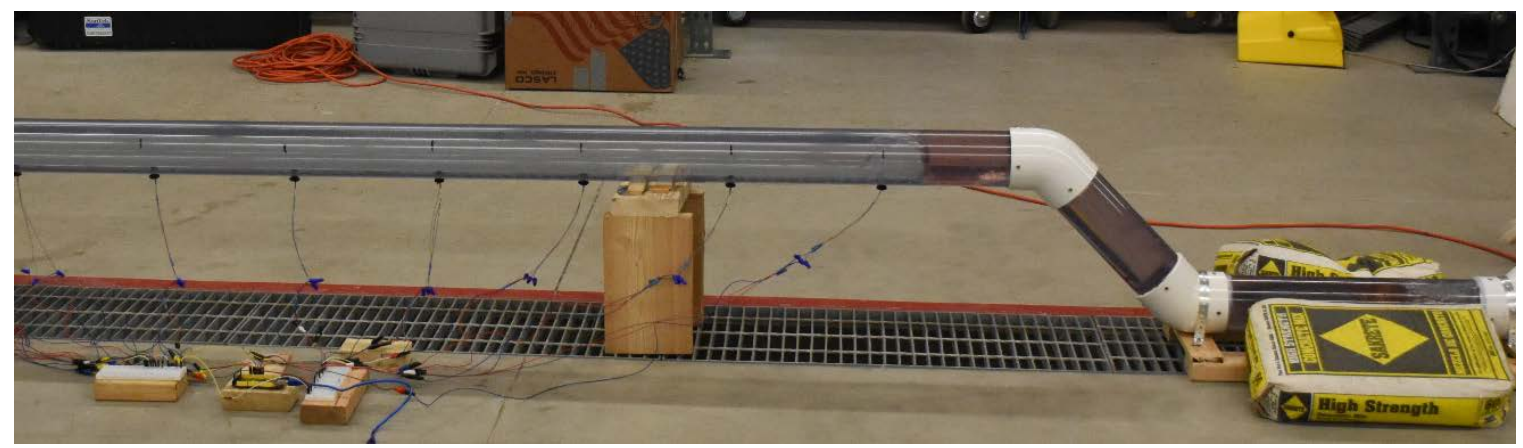

Figure 96: Wave profile generated by an inlet pressure of 2.75 psi

Sensors were connected to the 10-ft clear schedule 40 PVC pipe section attached to the outlet side of the U-section. Ten (five pairs) Adafruit Industries Optomax digital liquid level sensors (Figure 97) were used to measure the wave velocity. Figure 98 shows the sensor electrical connections.

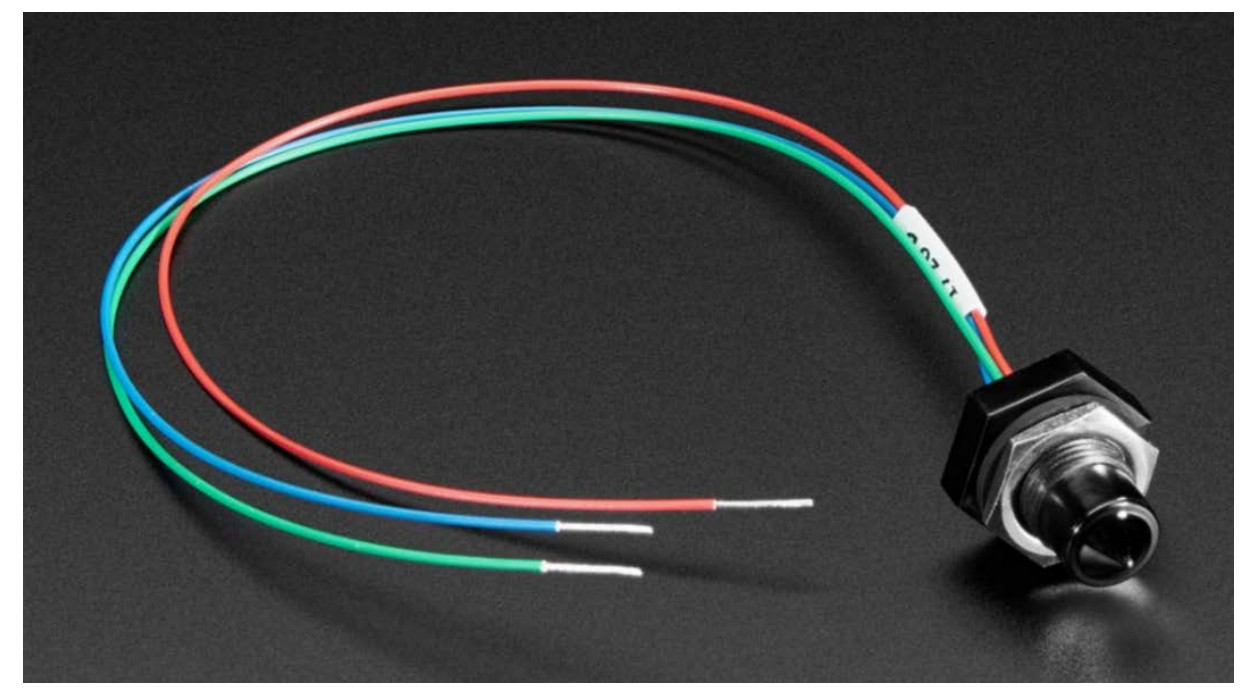

Figure 97: Optomax Digital Liquid Level Sensor 


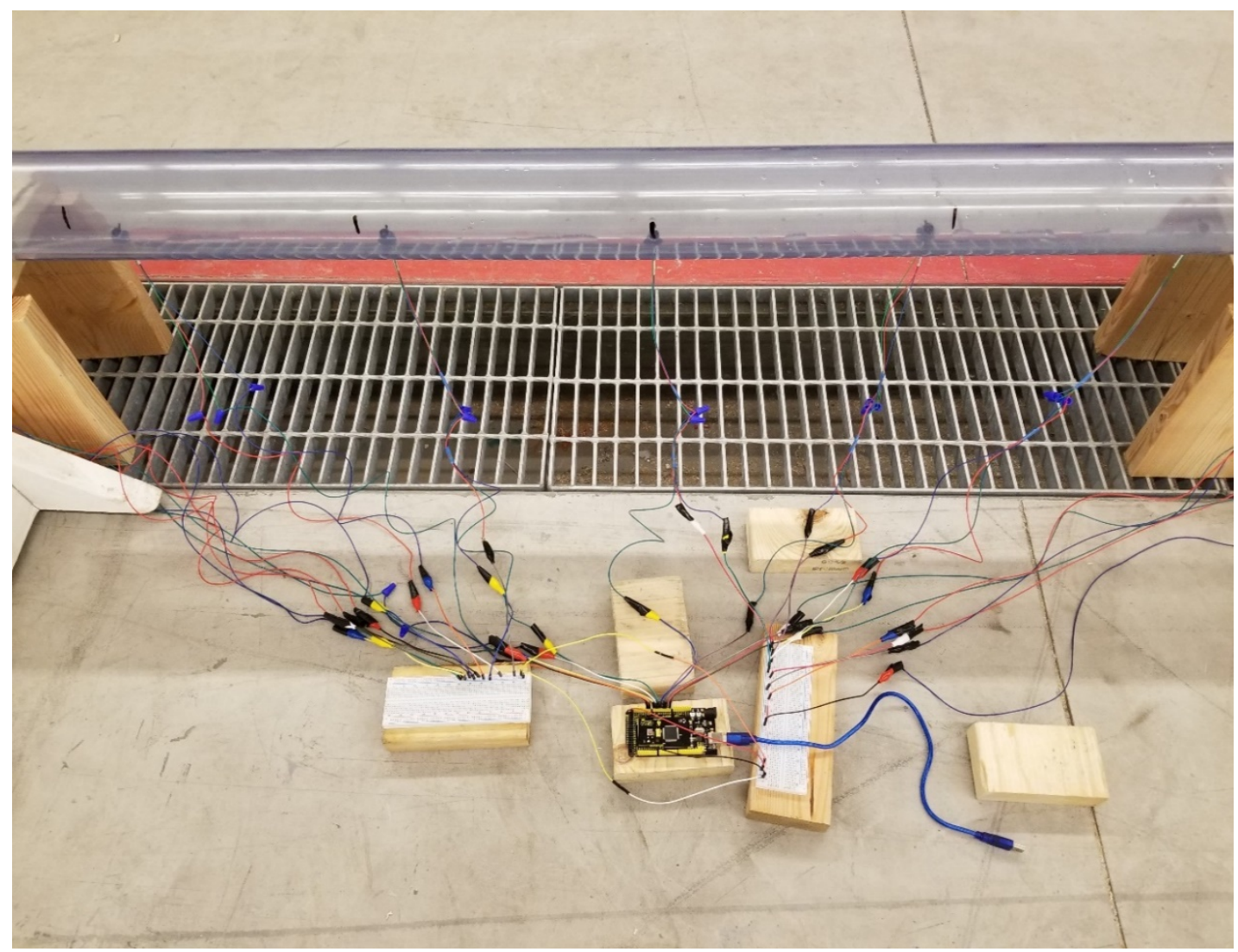

Figure 98: Sensor Electrical Connections

All individual sensors were powered and configured through a single Arduino mega microcontroller board, which in turn was connected to a computer. Figure 99 shows a flow chart of the code used to determine the wave velocity. Figure 100 shows the microcontroller being used in these processes. 

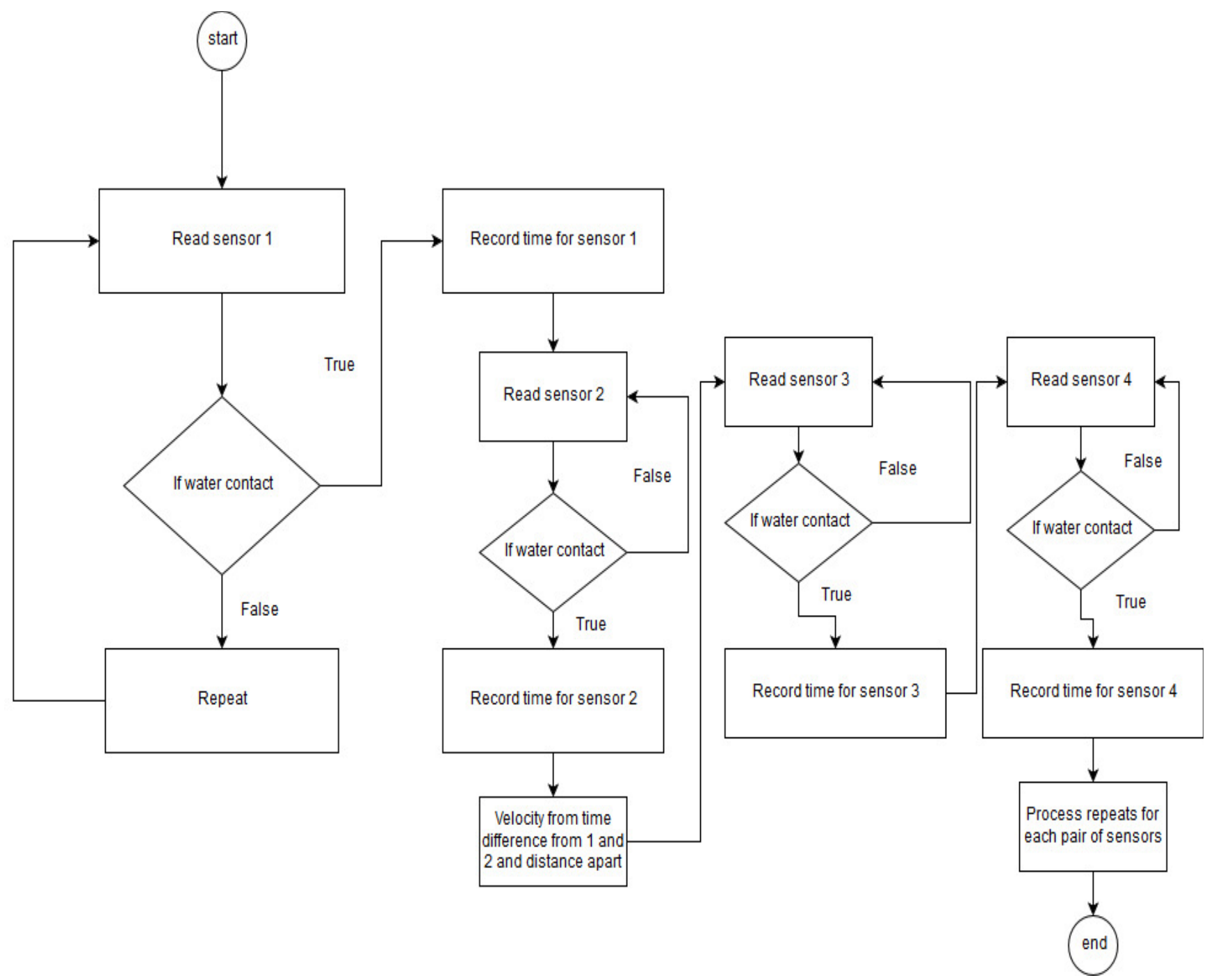

Figure 99: Flow Chart of the Code Used 

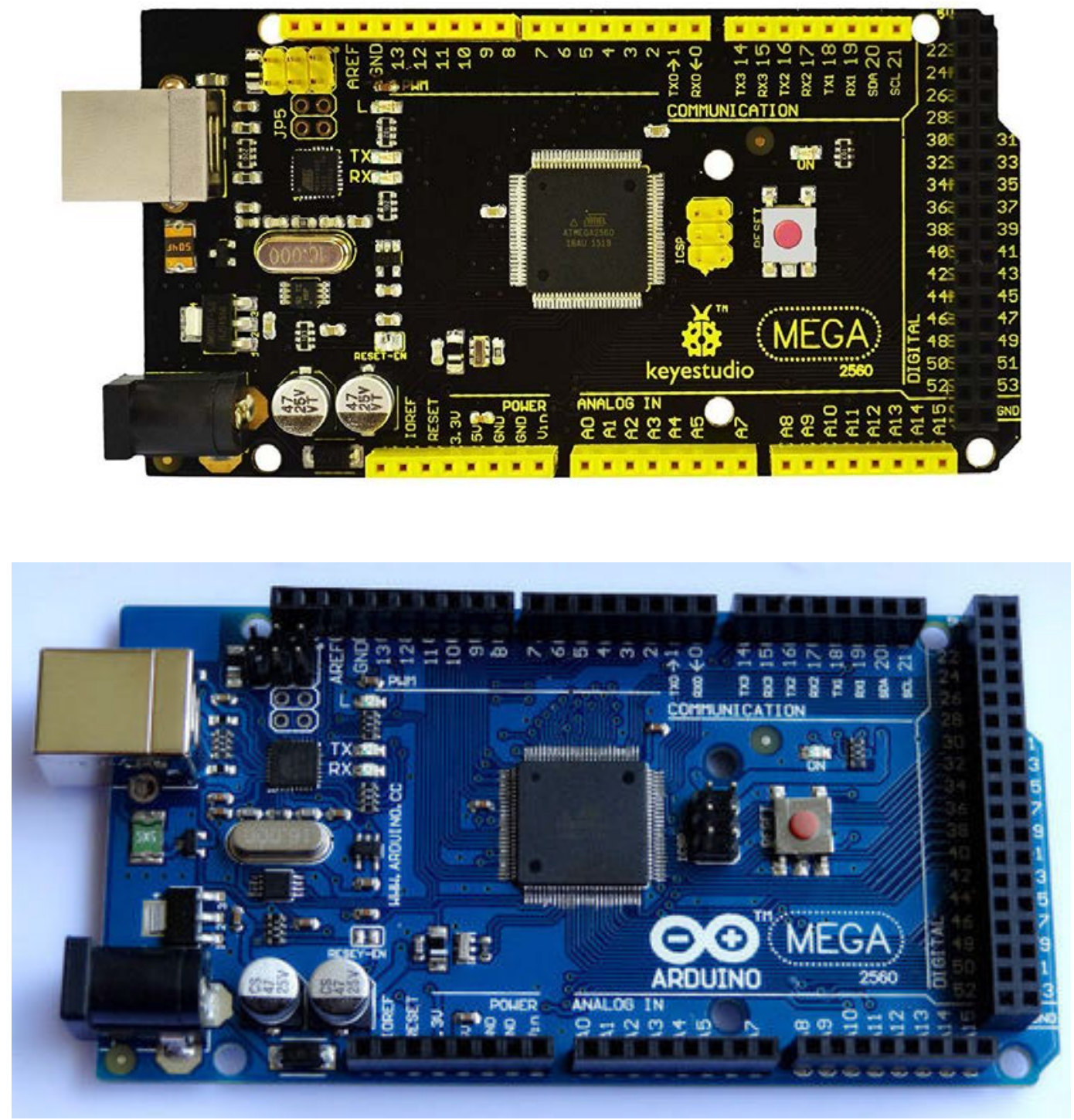

Figure 100: Arduino Mega 2560 Micro-controller

Results from the physical experimental study are shown in Table 33. The target velocity was 11.3 feet per second generated by a U-tube pressure of 1.09 psig within 0.53 seconds for a development length of $3 \mathrm{ft}$. The velocity measurement uncertainties are estimated to be $3.2 \%$. 
Table 33: Physical Experimental Study Results

\begin{tabular}{|c|c|c|c|c|c|c|c|c|c|}
\hline \multirow[b]{2}{*}{ Run } & \multirow[b]{2}{*}{$\begin{array}{c}\text { Chamber } \\
\text { Pressure (psig.) }\end{array}$} & \multirow[b]{2}{*}{$\begin{array}{l}\text { DL } \\
\text { (ft.) }\end{array}$} & \multirow[b]{2}{*}{ Time(s.) } & \multicolumn{5}{|c|}{ Velocity at locations: (ft./s) } & \multirow[b]{2}{*}{$\begin{array}{l}\text { Average U-tube } \\
\text { Pressure (psig.) }\end{array}$} \\
\hline & & & & $2 \mathrm{ft}$. & $4 \mathrm{ft}$. & $6 \mathrm{ft}$. & $8 \mathrm{ft}$. & $10 \mathrm{ft}$. & \\
\hline 1 & 10 & 3 & 0.53 & 22.73 & 12.05 & 38.46 & 27.78 & 25.94 & 2.89 \\
\hline 2 & 5 & 2 & 0.4 & 15.63 & 16.95 & 11.36 & 13.70 & 14.56 & 2.22 \\
\hline 3 & 5 & 3 & 0.53 & 28.57 & 11.36 & 16.95 & 16.67 & 16.60 & 1.85 \\
\hline 4 & 4 & 3 & 0.53 & 20.00 & 12.99 & 16.13 & 14.08 & 10.78 & 1.56 \\
\hline 5 & 3 & 2 & 0.4 & 12.35 & 12.50 & 12.99 & 12.82 & 14.56 & 1.6 \\
\hline 6 & 3 & 3 & 0.75 & 8.26 & 15.15 & 11.49 & 9.52 & 8.06 & 0.4 \\
\hline 7 & 3 & 3 & 0.75 & 13.70 & 13.25 & 10.42 & 12.50 & 11.53 & 1.1 \\
\hline 8 & 3 & 2.5 & 0.5 & 16.67 & 10.42 & 10.20 & 12.35 & 11.22 & 1.3 \\
\hline 9 & 3 & 3 & 0.58 & 16.13 & 11.49 & 12.20 & 10.10 & 11.22 & 1.1 \\
\hline 10 & 3 & 3 & 0.58 & 18.87 & 15.63 & 12.35 & 12.20 & 9.43 & 1.2 \\
\hline 11 & 3 & 3 & 0.53 & 14.08 & 15.63 & 9.71 & 12.20 & 11.53 & 1.2 \\
\hline 12 & 2.75 & 3 & 0.53 & 10.10 & 12.82 & 10.64 & 10.64 & 10.25 & 1.09 \\
\hline 13 & 2.75 & 3 & 0.53 & 11.63 & 12.82 & 12.35 & 10.10 & 8.30 & 1.11 \\
\hline 14 & 2.75 & 3 & 0.53 & 11.49 & 14.08 & 11.11 & 11.36 & 10.64 & 1.13 \\
\hline 15 & 2.75 & 3 & 0.53 & 11.24 & 12.20 & 11.76 & 9.35 & 12.39 & 1.09 \\
\hline 16 & 2.75 & 3 & 0.53 & 11.57 & 11.24 & 10.63 & 11.11 & 10.57 & 1.11 \\
\hline 17 & 2.75 & 3 & 0.53 & 11.76 & 13.16 & 10.42 & 10.35 & 9.71 & 1.14 \\
\hline 18 & 2.5 & 2 & 0.4 & 21.74 & 12.50 & 12.82 & 10.53 & 6.97 & 1.41 \\
\hline 19 & 1.5 & 3 & 0.63 & 6.25 & 10.87 & 10.20 & 8.26 & 15.37 & 0.82 \\
\hline 20 & 1.5 & 3 & 0.75 & 11.63 & 14.49 & 12.35 & 11.49 & 9.76 & 1.19 \\
\hline 21 & 1.5 & 3 & 0.75 & 7.75 & 7.94 & 7.63 & 6.49 & 6.10 & 0.5 \\
\hline 22 & 1.5 & 3 & 0.58 & 8.33 & 8.06 & 8.33 & 7.19 & 7.76 & 0.68 \\
\hline
\end{tabular}

Using an inlet pressure of 2.75 psig yielded a nearly constant velocity, which is close to what was expected. Figure 101 shows the velocities yielded by a chamber pressure of 2.75 psig at selected locations on the downstream pipe. The fifth test simulation with a chamber pressure of 2.75 psig yielded the best result with near constant velocities at all locations. For this simulation, it can be observed that water accelerated with a peak velocity of around $11.5 \mathrm{ft}$. /s at the location of two feet and then decelerated slowly. Another small peak velocity is observed at the location of eight feet. Gravity acts on the wave front and produces a tongue in the bottom portion as shown in Figure 102 which might be a reason for the peak velocity as water is accelerated by gravity. In another perspective, as the tongue is produced, the wetted perimeter of the water jet is reduced, when compared with the jet with a vertical wave front, as the top portion of the wave is dragged down by gravity. With the smaller surface area in contact, water might have been additionally accelerated due to the acting pressure. 


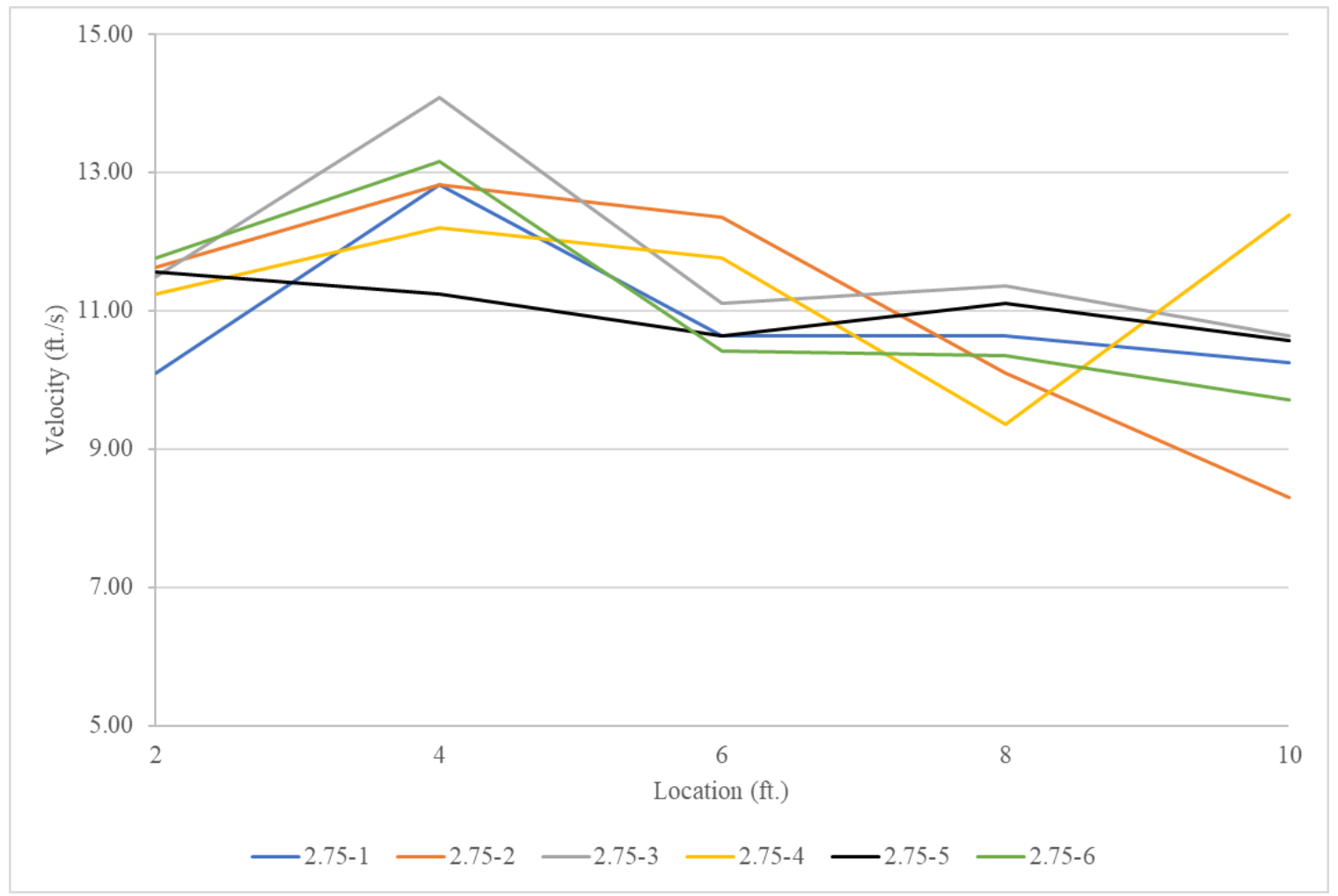

Figure 101: Velocity results.

The chamber pressures and their respective U-tube pressures are compared and presented in Figure 103. A relation between these pressures can be generated almost accurately with an equation and additionally, a correlation coefficient has been generated as shown in the figure. The correlation coefficient is quite close to unity indicating that the regression line almost perfectly fits the data.

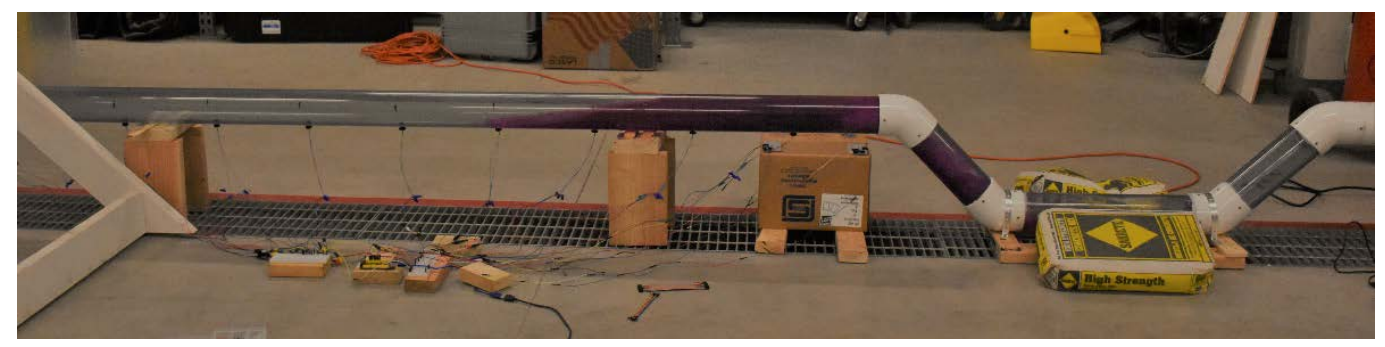

Figure 102: Tongue produced by the wave. 


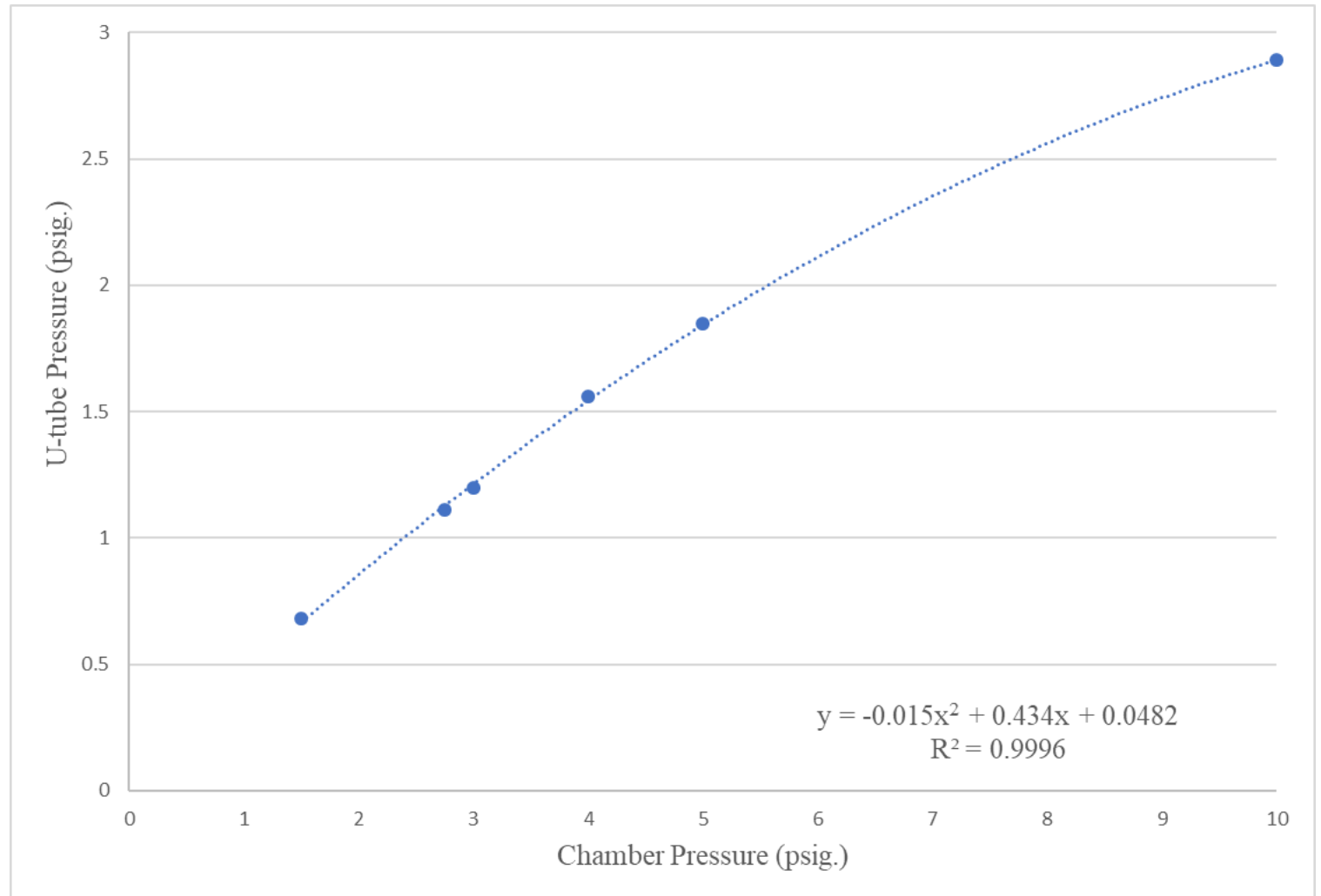

Figure 103: Chamber pressure vs U-tube pressure

These results indicate that the physical and theoretical experiments are similar to one another as shown in Table 34. This verifies the workability of air pressure to generate the motive force on the water, and, similarly, the velocity sensors proved to work very well with the ability to records velocities up to 28 feet per second.

Table 34: Physical vs Theoretical Results for a Chamber Pressure of 2.75 psig (run 5)

\begin{tabular}{|c|c|c|}
\hline Results & Theoretical & Physical \\
\hline U-tube pressure (psig) & 1.09 & 1.11 \\
\hline Velocity (ft. /s) & 11.3 & $11.57,11.24,10.63,11.11$,and 10.57 \\
\hline
\end{tabular}




\section{WAVE IMPACT SIMULATION DEVICE STRUCTURAL ANALYSIS}

This section discusses structural analysis associated with the WISD (Figure 104). Results of the software validation indicated that thinner plates could be used if the spanning width was decreased. Plates spanning the entire 10-ft. width would require additional thickness, but they would be less invasive on the developing wave than multiple sections per channel. Design iterations were used to identify the advantages and disadvantages of each option.

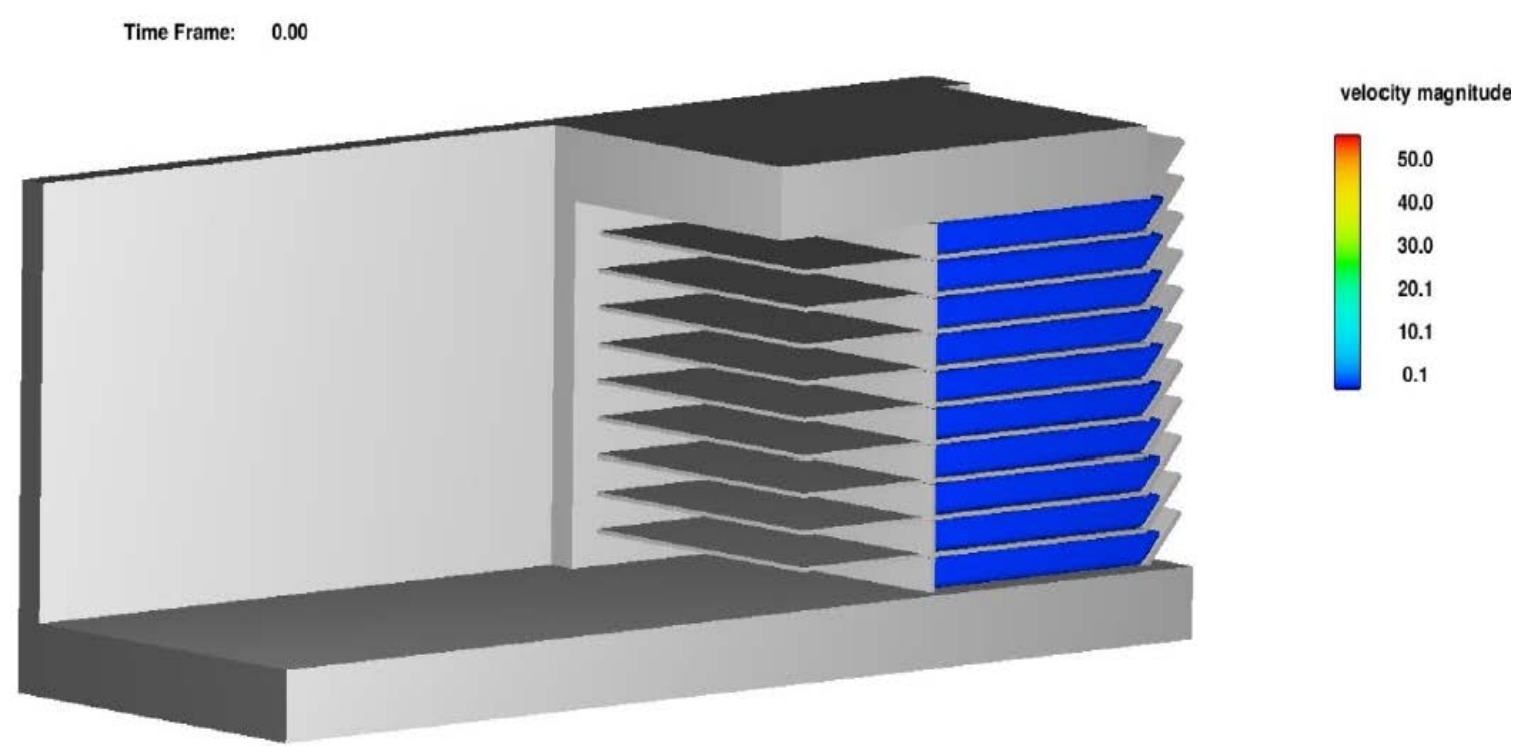

Figure 104: Wave Impact Simulation Device design.

Plate Design Iteration 1: Four 2-1/2' Plates per 10’ Wide Channel

Model Details: $\quad$ Four 2.5’ x 10’ x 3/32”' A36 steel plates were designed to span the 10-ft. wide channel. A single plate was modeled with the long edges fixed from translation and rotation. A total of 36 plates would be required for the entire prototype, (see Figure 105). A uniform pressure of 0.433 psi was applied over the face of the plate.

FEA Results: The maximum von-Mises stress was observed to be 17,446 psi, (see Figure 106), with a maximum deflection of 0.044”, (see Figure 107).

Remarks: $\quad$ The maximum stress did not exceed the 36,000 psi yield stress of A36 steel, and the 0.044” deflection was considered minimal. However, it was unclear how dividing the $10 \mathrm{ft}$. channel into four separate 2-1/2 ft. sections might affect the target flow profile. The vertical flow profile appeared unchanged from a single channel of $10-\mathrm{ft}$ width but installing supports for each section was considered a disadvantage. The team agreed more options should be explored. 


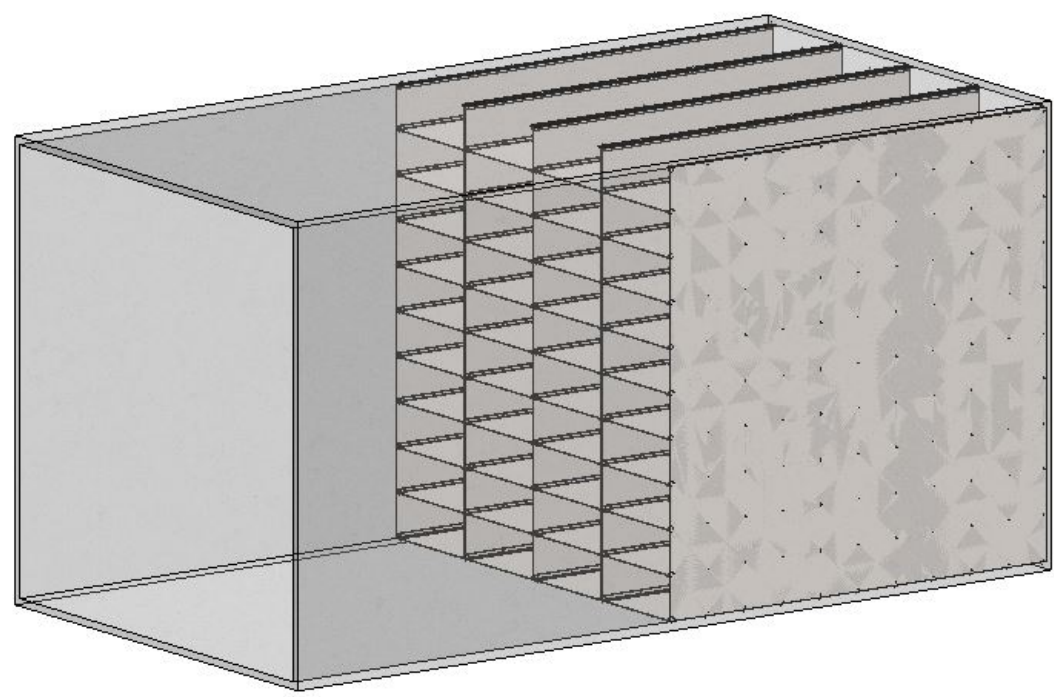

Figure 105. Overview of the 2-1/2' x 10’ x 3/32” plate system.

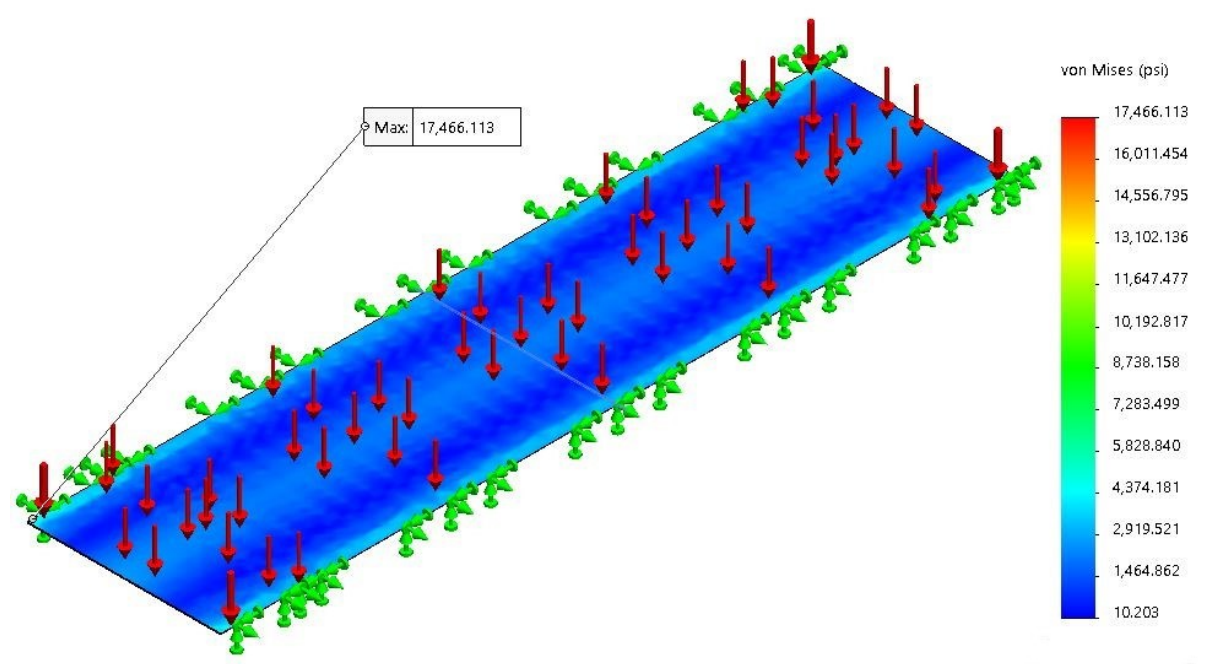

Figure 106. Von-Mises stress of a 2-1/2' x 10' x 3/32" plate with long edges fixed under 0.433 psi. 


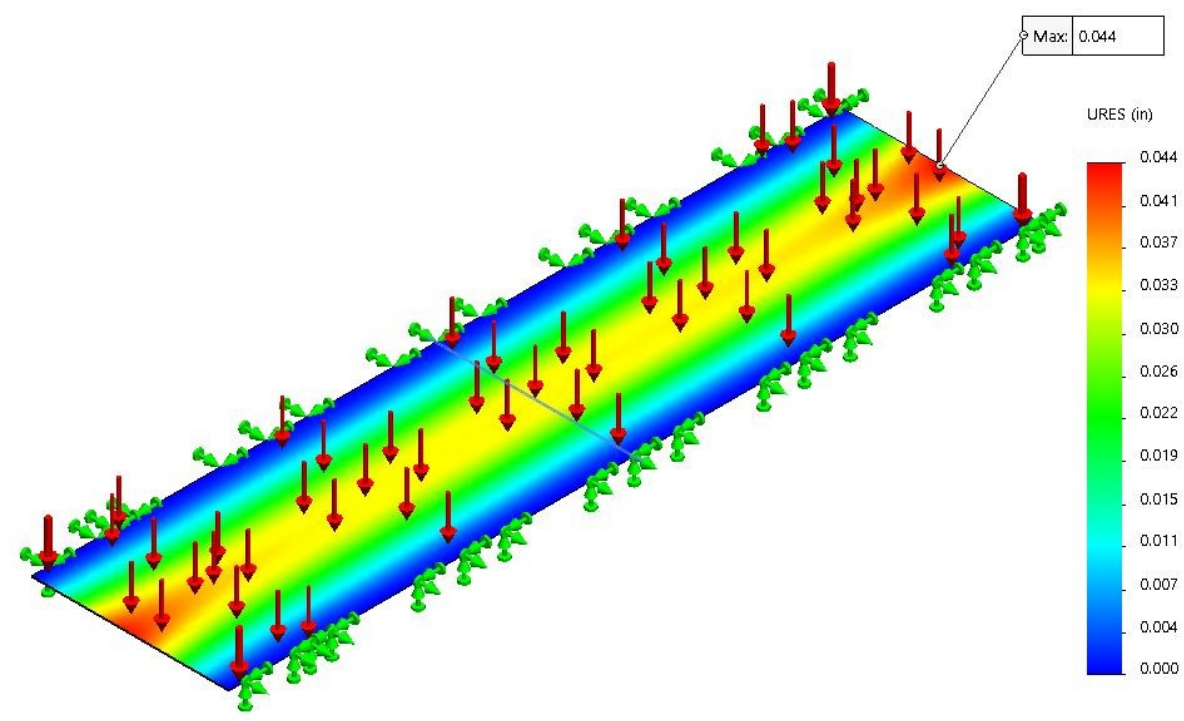

Figure 107. Deflection of a 2-1/2' x 10' x 3/32" plate with long edges fixed under 0.433 psi.

Plate Design Iteration 2: A Single 10' Wide Plate per 10' Channel

Model Details: $\quad$ A 10' x 10' x 1” thick plate was modeled with long edges fixed. A total of 9 plates would be required for the reservoir section of the prototype, (see Figure 108). A uniform pressure of 0.433 psi was applied over the face of the plate.

FEA Results: $\quad$ Maximum von-Mises stress was 2,743 psi (see Figure 109). Maximum deflection was 0.099” (see Figure 110).

Remarks: $\quad$ Each 10 ’ x 10’ x 1” plate would weigh approximately 4,084 lbs., a significant issue for installation and safety issues. A 1” thick plate was also scrutinized as being highly disruptive to a developing flow profile. The solution was to use a thinner plate and investigate different options for supporting it. 


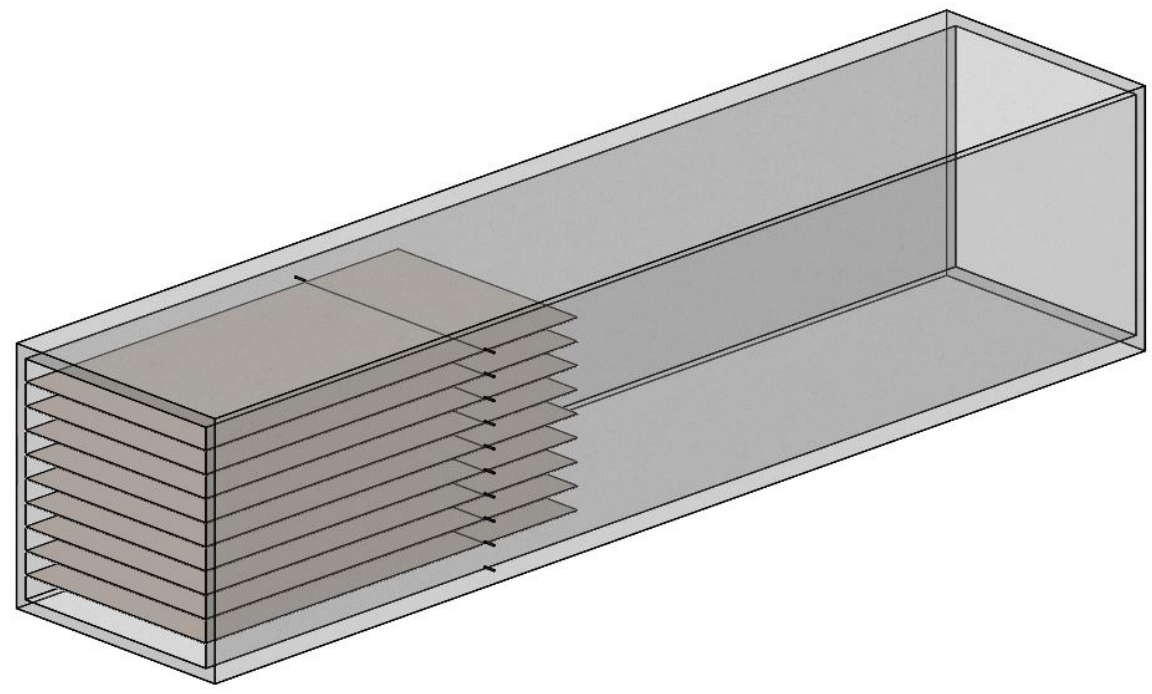

Figure 108. Overview of the 10 ' x 10' x 1” plate system.

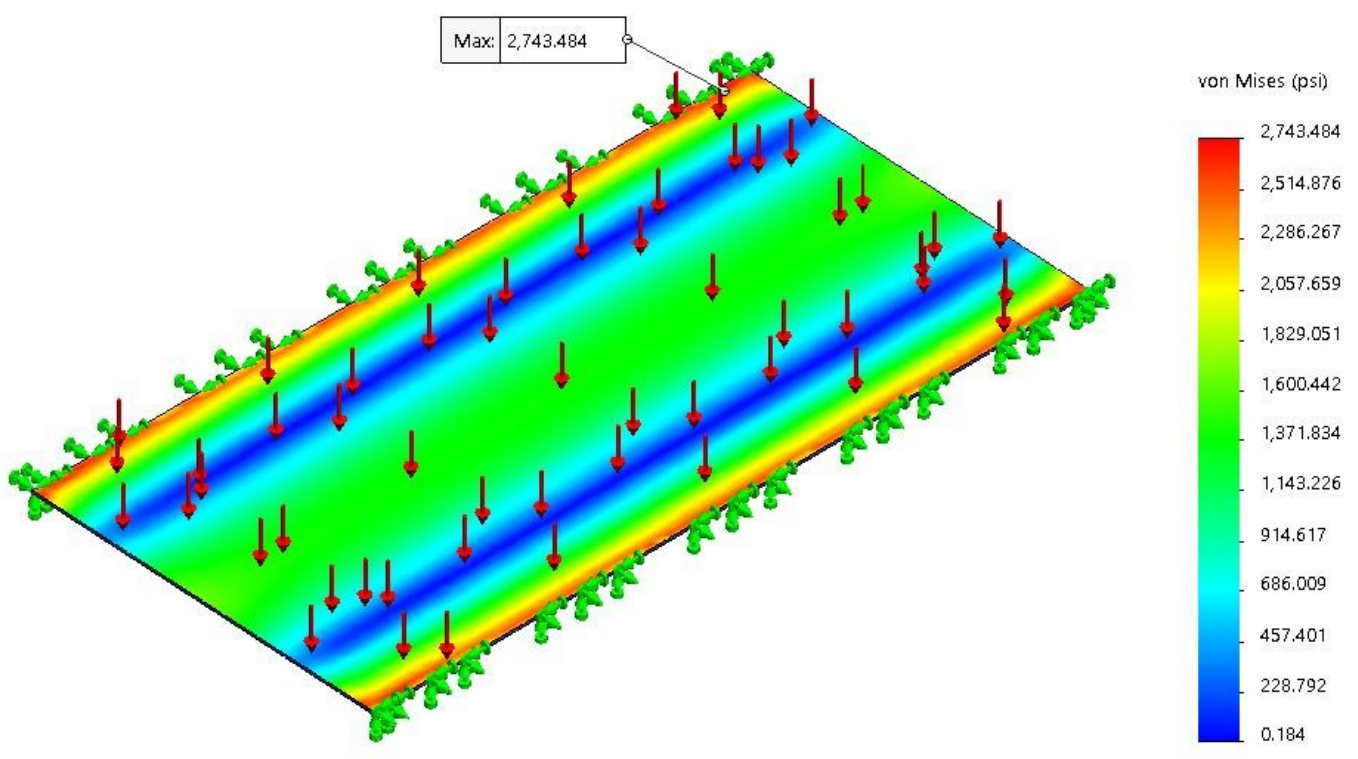

Figure 109. Von-Mises stress of a 10' x 10' x 1" plate with long edges fixed under 0.433 psi. 


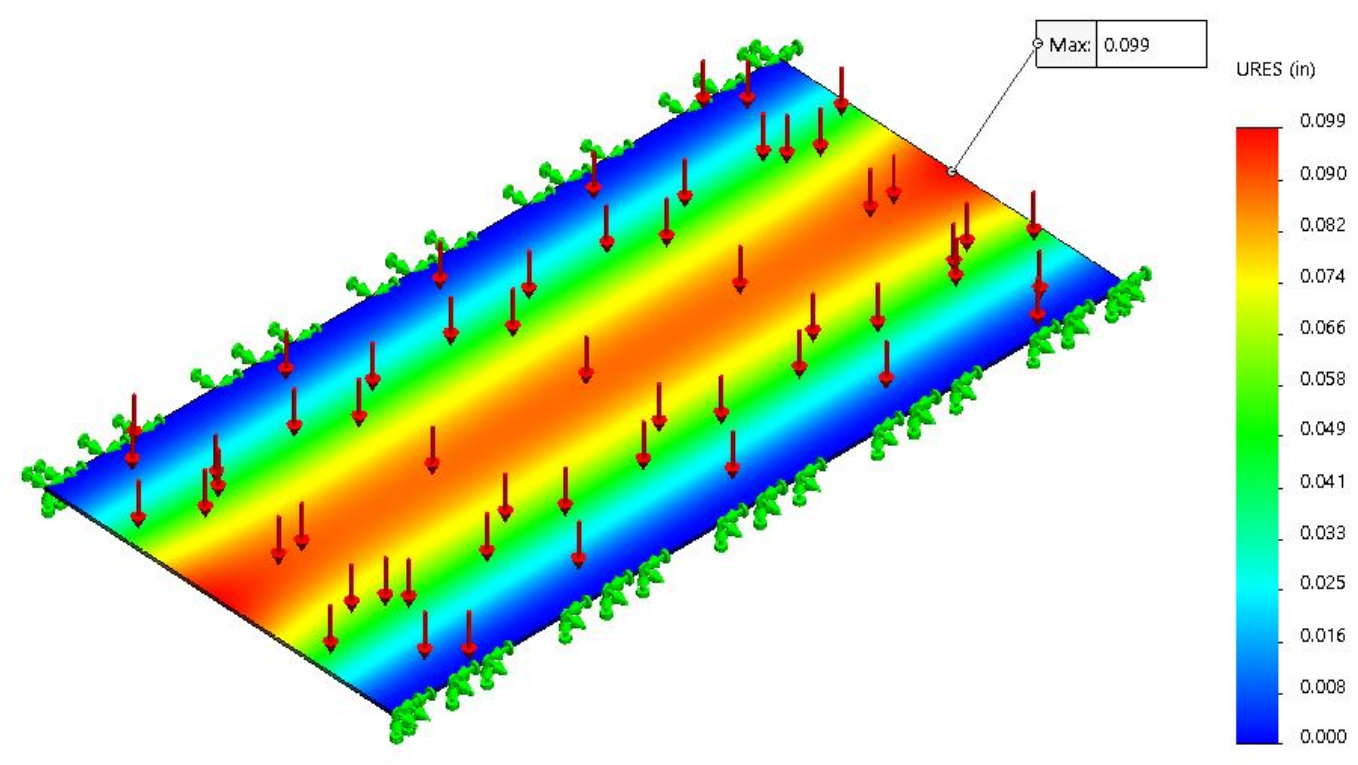

Figure 110. Deflection stress of a 10' x 10' x 1" plate with long edges fixed under 0.433 psi.

Plate Design Iteration 3: Plate Thickness Decreased and Number of Fixed Edges Increased

Model Details: A $10^{\prime}$ x 10' x 3/8” plate was modeled with three sides fixed in place. A uniform pressure of 0.433 psi was applied over the face of the plate.

FEA Results: Maximum von-Mises stress was 19,327 psi (see Figure 111). Maximum deflection was 1.852” (see Figure 112).

Remarks: $\quad$ The 19,327 psi maximum stress did not exceed the 36,000 psi yield stress of A36 steel, but the deflection was substantial at 1.852”. Additional support was deemed necessary. 

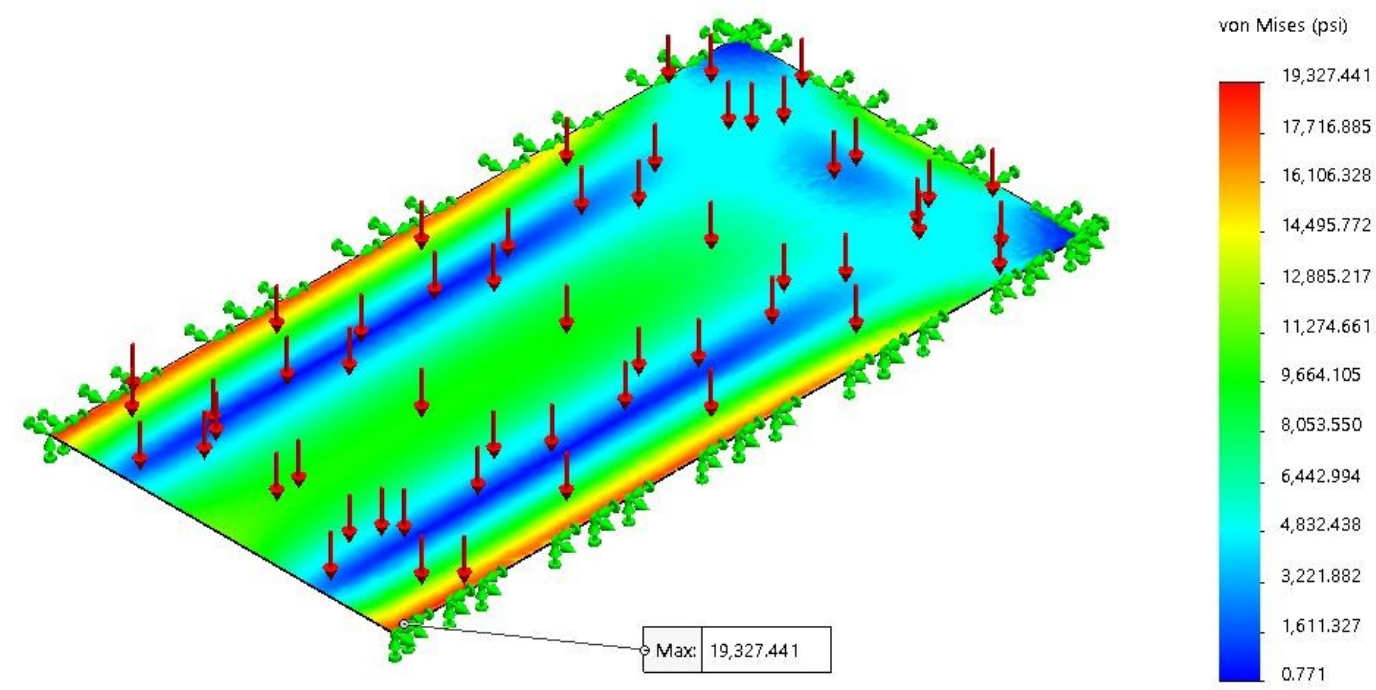

Figure 111. Von-Mises stress of a 10' x 10' x 3/8" plate with three fixed edges under 0.433 psi.

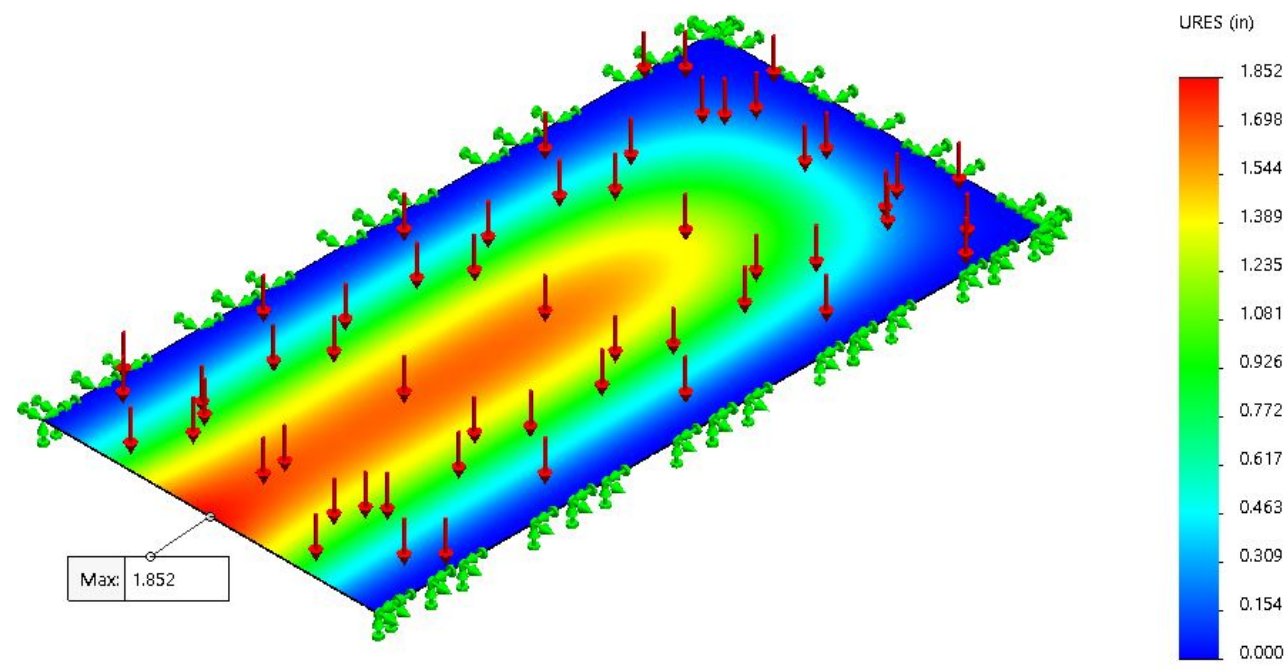

Figure 112. Deflection of a 10' x 10' x 3/8" plate with three fixed edges under 0.433 psi.

Plate Design Iteration 4: Fixed Edges Increased to all Four Sides of Plate

Model Details: $\quad$ A $10^{\prime}$ x 10' x 3/8” thick plate was modeled with all four sides fixed. A uniform pressure of 0.433 psi was applied over the face of the plate.

FEA Results: $\quad$ Maximum von-Mises stress was 18,098 psi, (see Figure 113). Maximum deflection was 1.630” (see Figure 114). 19,327 psi (see Figure 111). 
Remarks: $\quad$ The maximum stress was reduced from 19,327 to 18,098 psi, and the deflection was reduced from 1.852” to 1.630”. The deflection was still considered excessive. The team began research on thin supports to decrease deflection at the center of the plate.

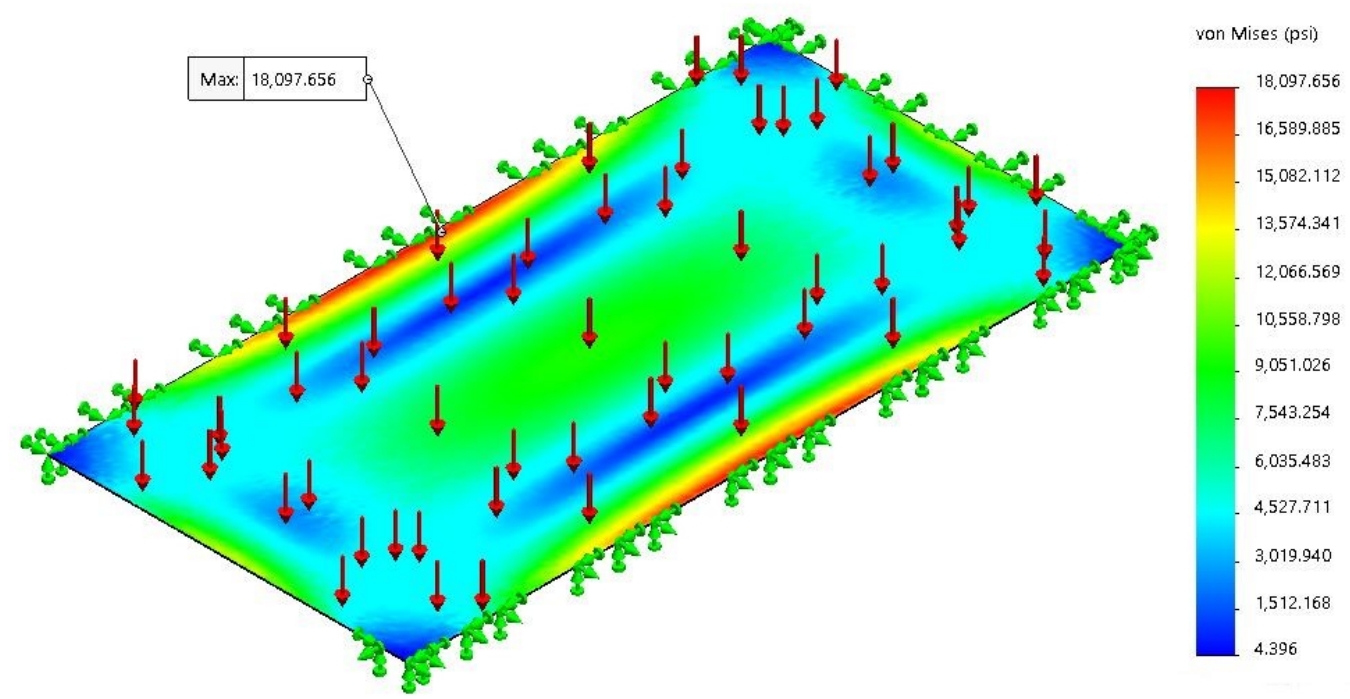

Figure 113. Von-Mises stress of a 10' x 10' x 3/8" plate with three fixed edges under 0.433 psi.

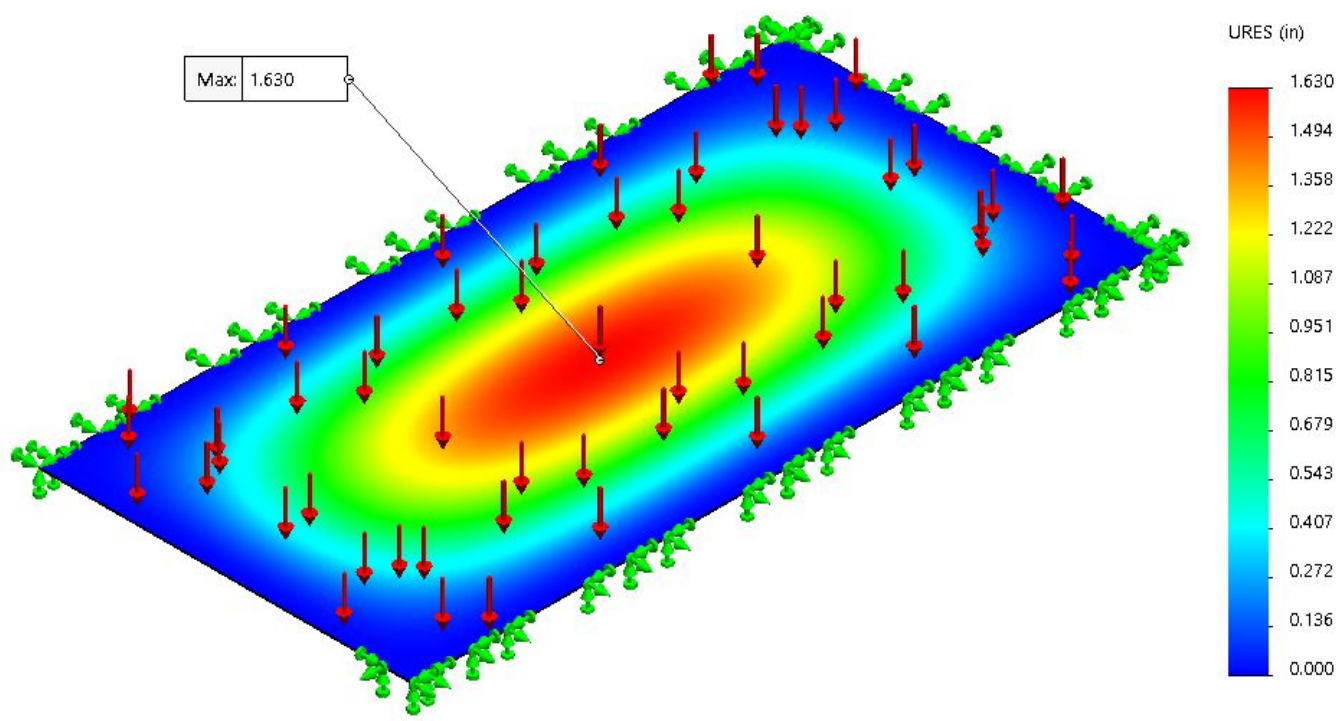

Figure 114. Deflection of a 10' x 10' x 1" plate with three fixed edges under 0.433 psi.

It was at this time in the design process that the concurrent FLOW-3D research being performed which showed that increasing the hydrostatic head from $1 \mathrm{ft}$ to $3 \mathrm{ft}$. would aid in the development of a near vertical flow profile. The FLOW-3D simulation also indicated as much as 20 psi of air pressure would be required to accelerate the water from stagnation to a constant $25.4 \mathrm{ft} / \mathrm{s}$ velocity before it exists the conduit.

The change in design parameters from 0.433 psi to approximately 21.3 psi substantially increased the difficulty in developing an adequately thin, yet adequately strong prototype plate. The need for additional plate 
supports became imperative. It was assumed that adding supports to the reservoir section would have less of an impact on the target velocity profile than adding supports to the plates located downstream of the gate system. Furthermore, the water contained in the post-gate section would not be static. It would be in constant motion when acting upon by the 20 psi horizontal, pneumatic force. As such, the design focus was shifted from the reservoir plates to the post-gate plates to determine the stress and deflection behavior under these new parameters.

\section{Plate Design Iteration 5: Investigation of Static vs. Dynamic Effects on Post Gate Plates}

Model Details: $\quad$ Nine 10' x 5' x 1/2" plates were modeled with three edges fixed in place (exit edge remained simply supported). All nine plates were included in the simulation to observe the overall behavior of the plate system (see Figure 115). Two FEA studies were performed to compare the behavior of all nine plates when subjected to a static, then dynamic load. For the static study, a uniform 1.3 psi pressure was applied vertically to the top plane of each plate. The dynamic study included a non-linear, time-dependent study based on the 20 psi horizontal force also acting on the water column.

FEA Results: $\quad$ The static analysis showed a maximum von-Mises stress of 8,864 psi would develop in all nine plates when a 1.3 psi pressure is applied, (see Figure 116). The maximum deflection was 0.413 ” (see Figure 117). In the time-dependent analysis, the maximum von-Mises stress was 8,372 psi (see Figure 118), and the maximum deflection was 0.442 ” for the 20 psi horizontal and 1.3 psi vertical loads (see Figure 119 and Figure 120).

Remarks: $\quad$ The time-dependent simulation used 67 time steps from the onset of deflection to cessation. Figure 119 and Figure 120 each contain six time step images showing the deflection at each respective time step. The stress to each plate at maximum deflection is shown in Figure 118. A comparison between the two studies confirmed flowing water through all nine plates minimally lessens the stress experienced by each plate and actually increases the deflection. In either case, the nearly 1/2" deflection was not considered acceptable. The plate thickness could not be increased any further, and the investigation into an alternative support system continued. 


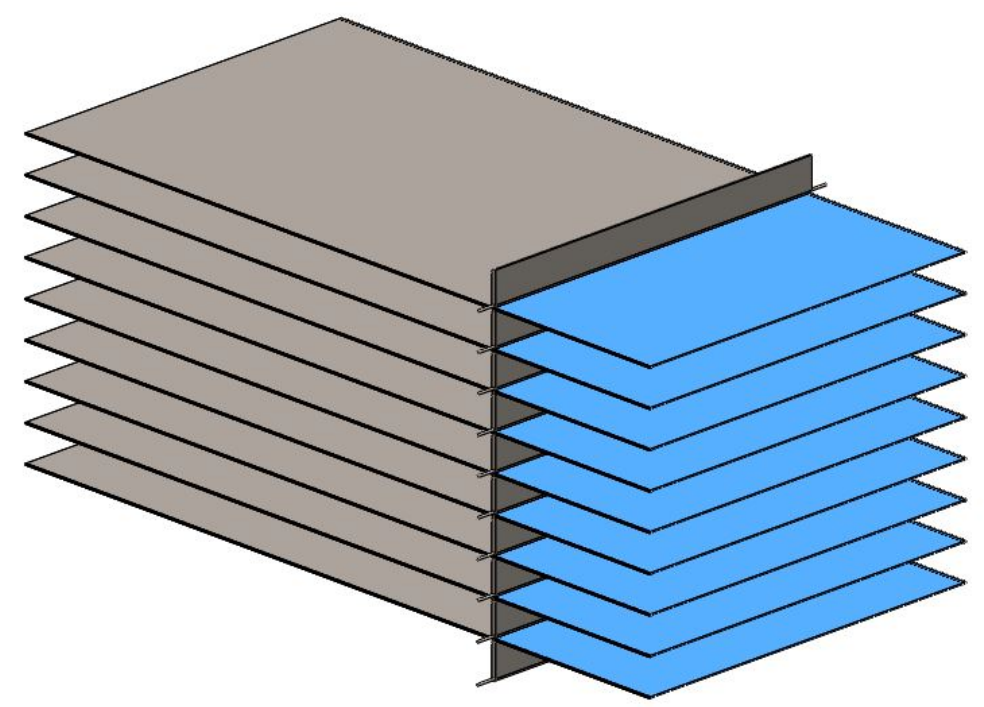

Figure 115 . Overview of the $10^{\prime}$ x 5’ x 1/2" post-gate system.

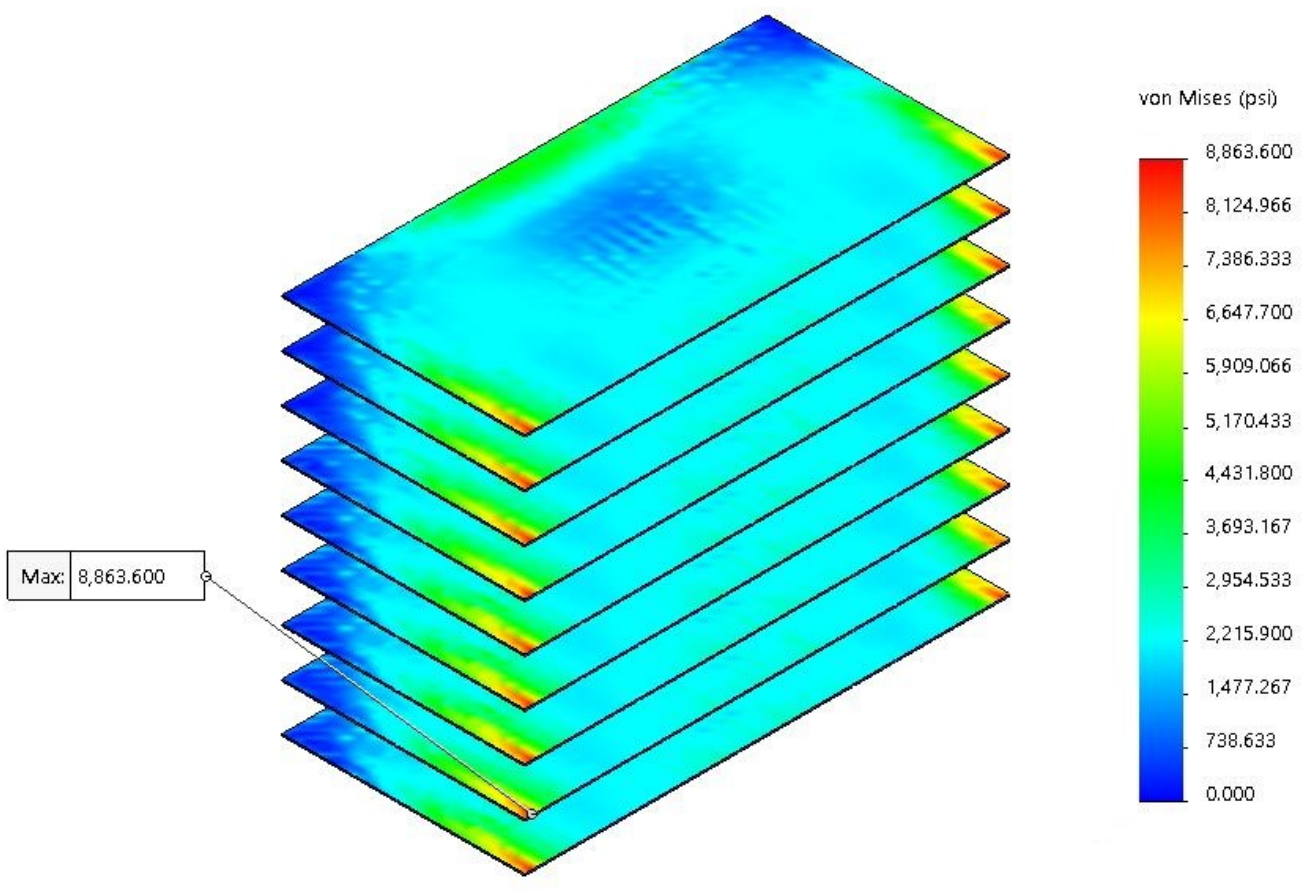

Figure 116. Von-Mises stress for nine 10' x 5' x 1/2" plates under a 1.3 psi static load. 


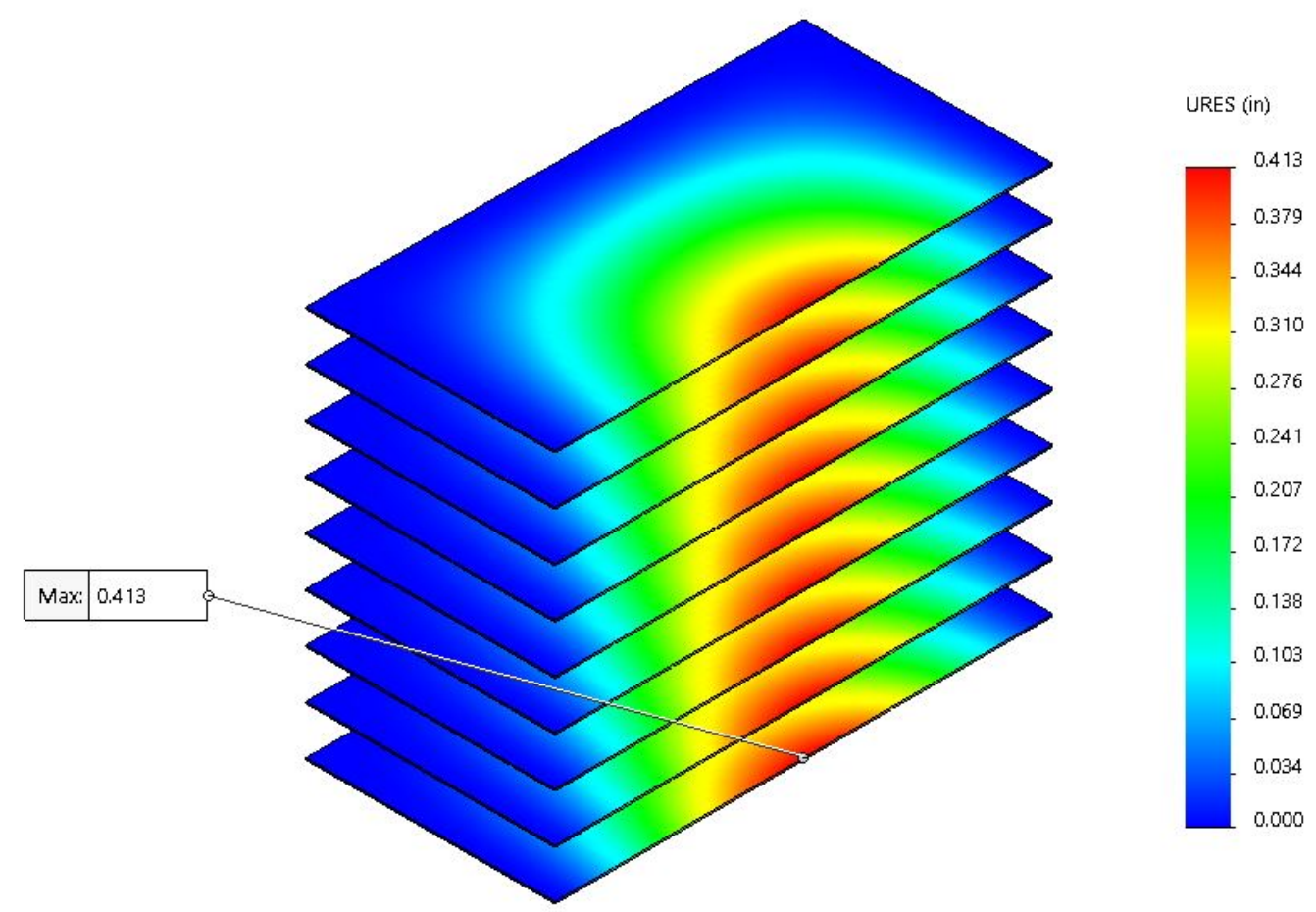

Figure 117. Deflection for the nine 10' x 5' x 1/2" plates under a 1.3 psi static load.

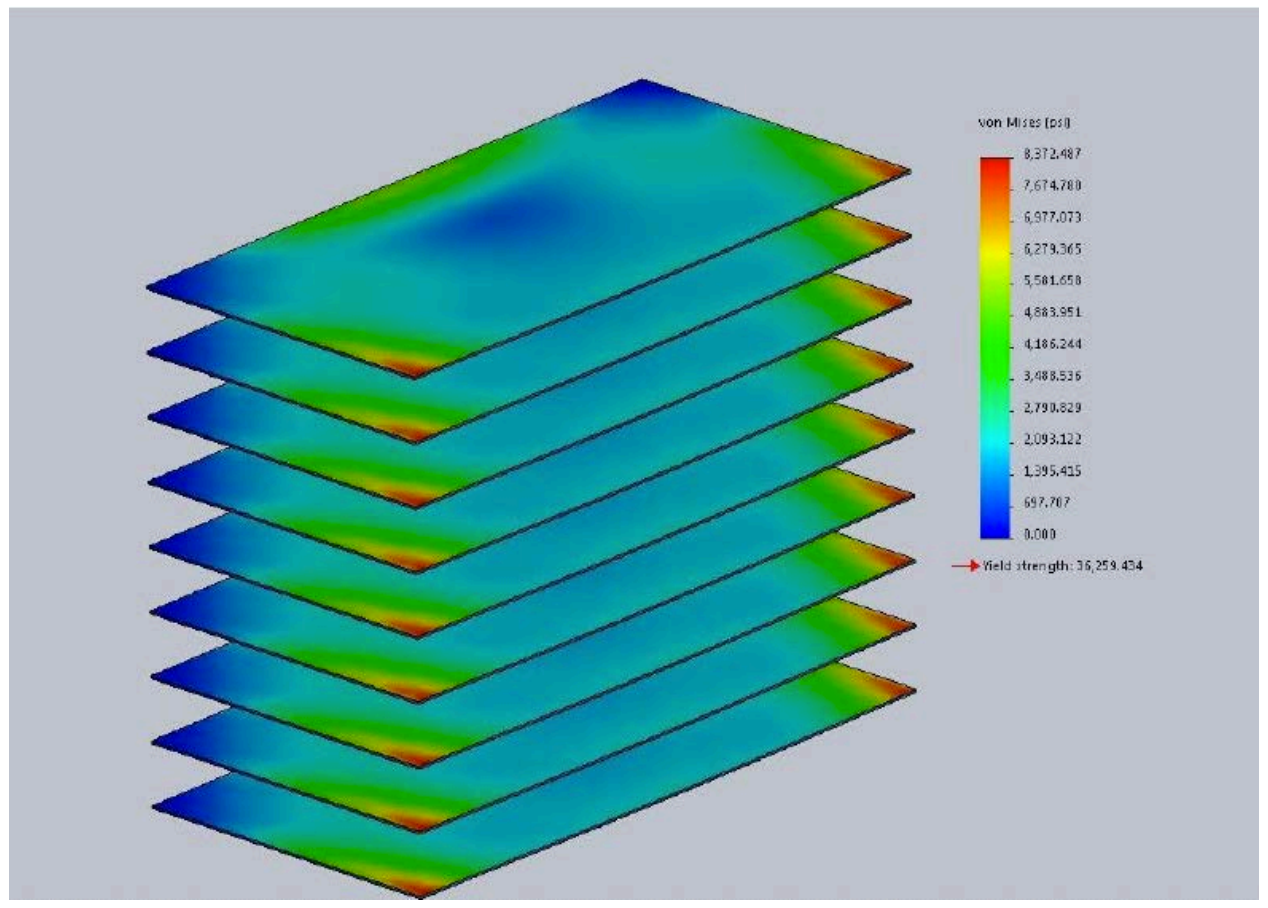

Figure 118. Von-Mises stress for the nine $10^{\prime}$ x 5' x 1/2" plates under static and dynamic loading. 


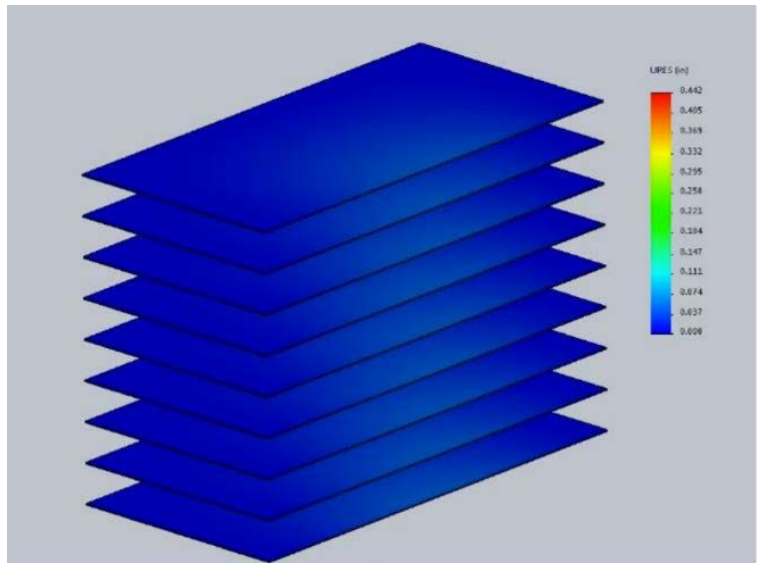

$t \approx 0.01 \mathrm{~s}$, max deflection $\approx 0.053 \mathrm{in}$.

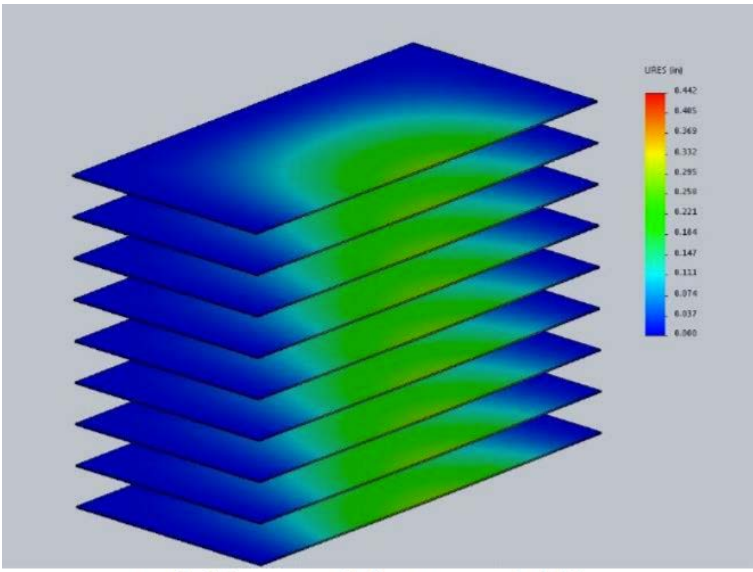

$t \approx 0.10$ s, max deflection $\approx 0.278$ in.

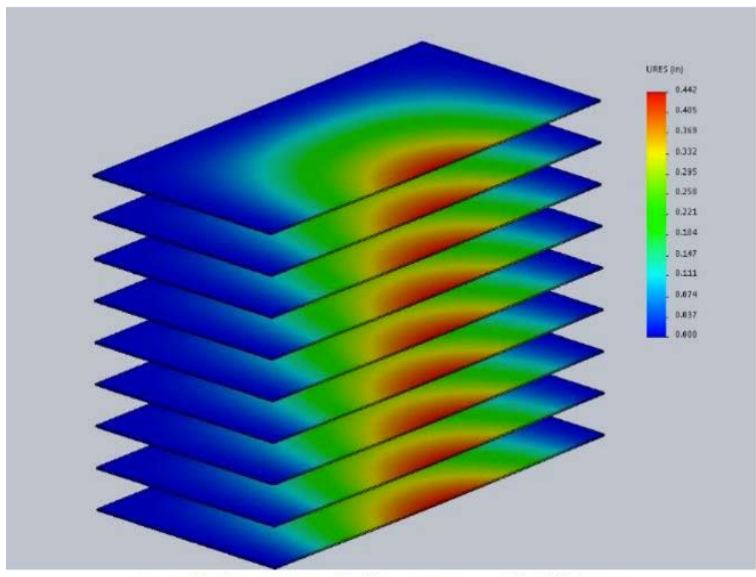

$t \approx 0.2 \mathrm{~s}$, max deflection $=0.442 \mathrm{in}$.

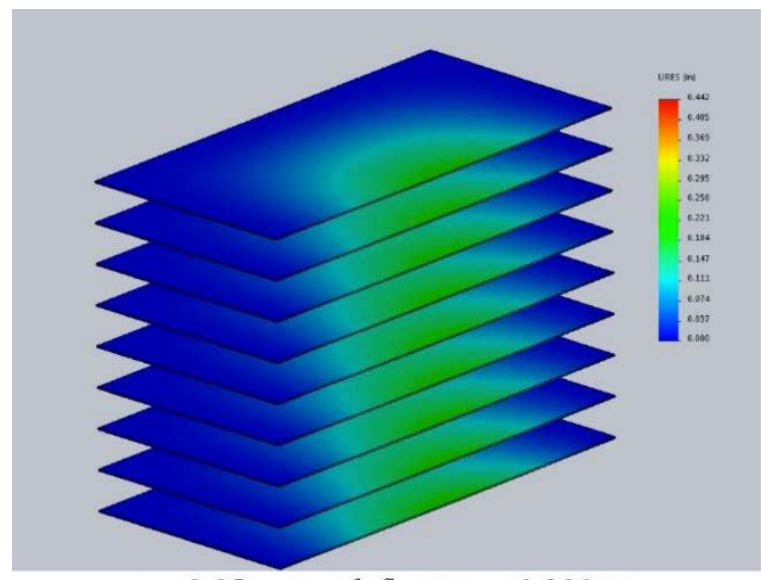

$t \approx 0.05 \mathrm{~s}$, max deflection $\approx 0.201 \mathrm{in}$.

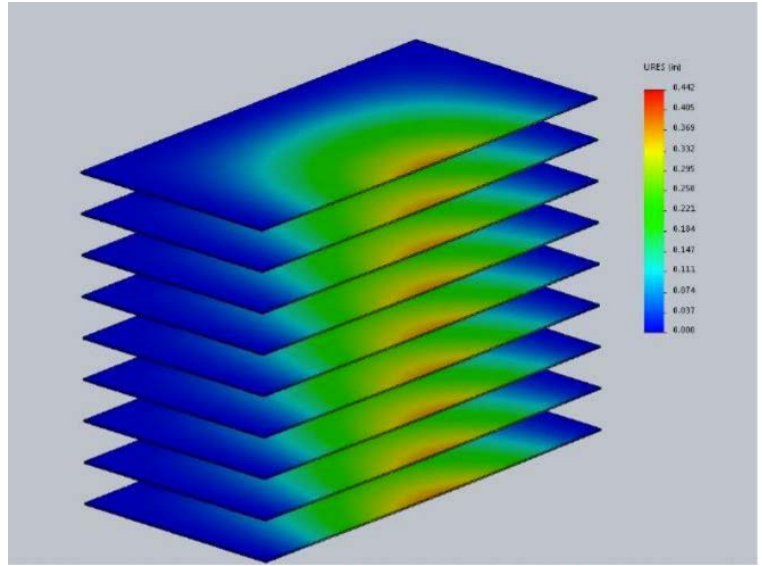

$t \approx 0.15 \mathrm{~s}$, max deflection $\approx 0.370 \mathrm{in}$.

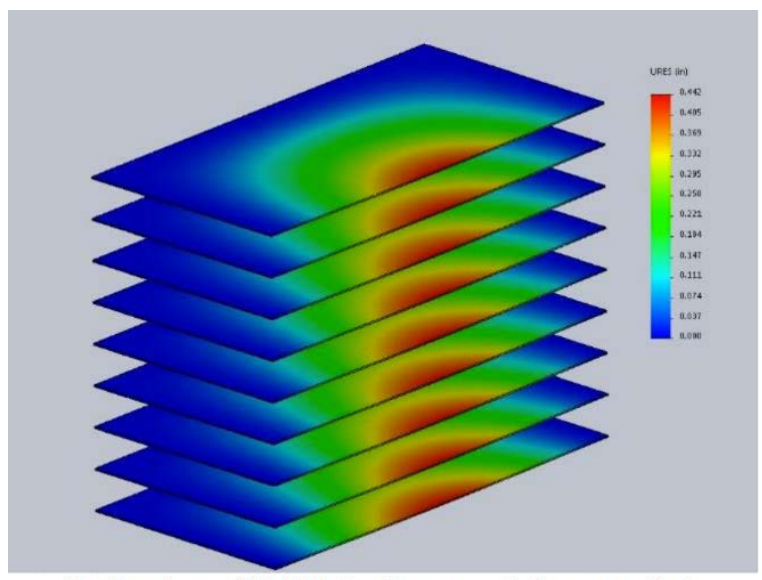

Deflection of $0.442 \mathrm{in}$. from $t \approx 0.2 \mathrm{~s}$ to $t \approx 0.6 \mathrm{~s}$

Figure 119. Onset of deflection of the $10^{\prime}$ x 5' x 1/2" plates under static and dynamic loading.

$(t=0.01$ to $t=0.2$ seconds $)$ 


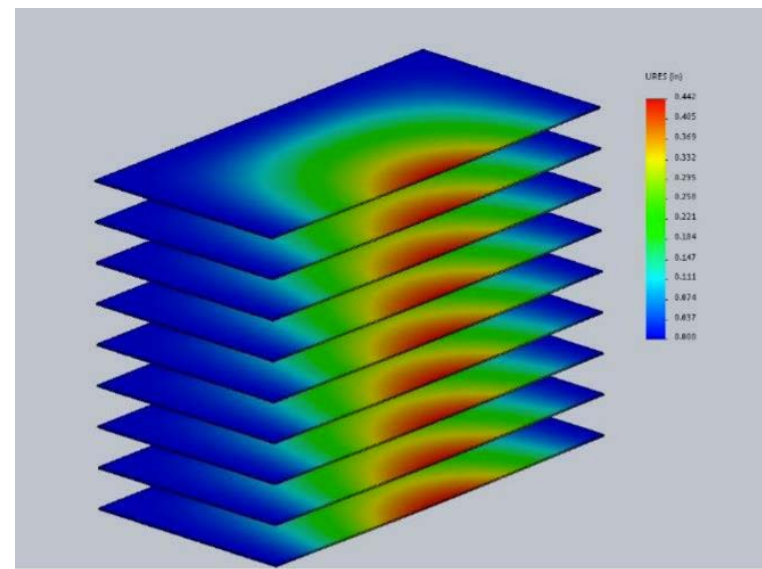

$t \approx 0.59 \mathrm{~s}$, max deflection $=0.442 \mathrm{in}$.

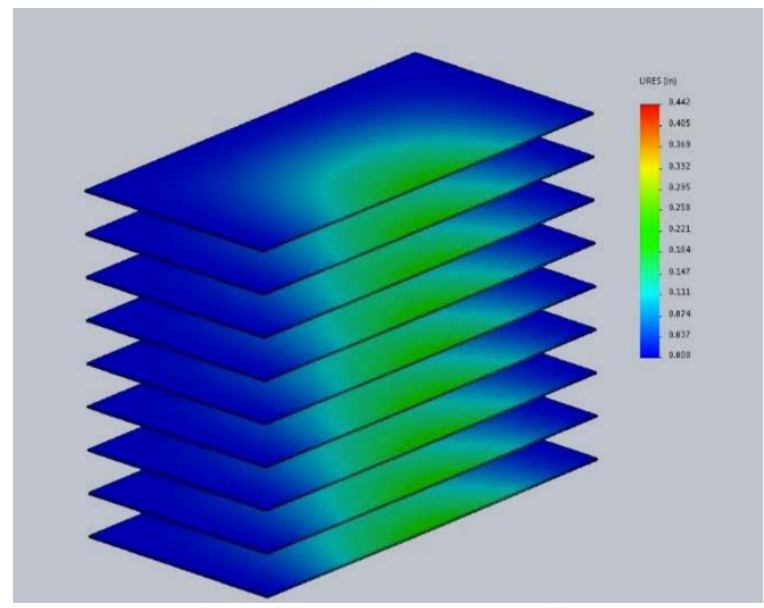

$t \approx 0.71 \mathrm{~s}$, max deflection $\approx 0.201 \mathrm{in}$.

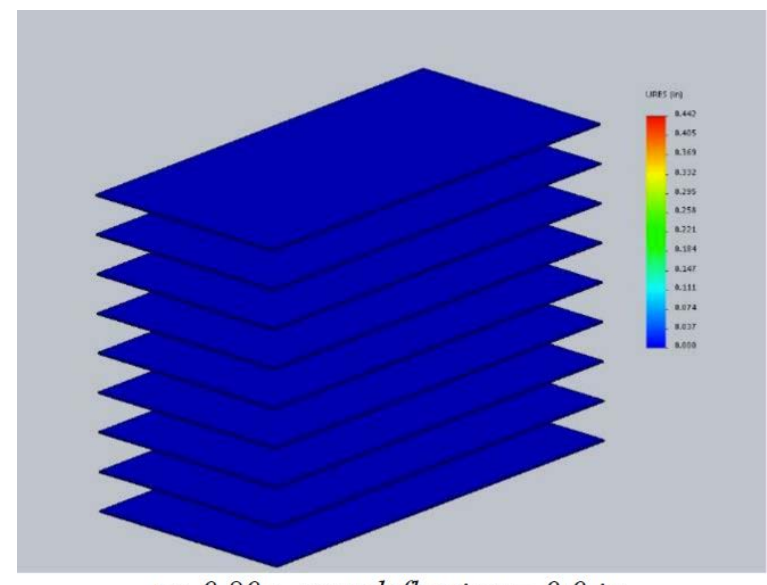

$t \approx 0.80 \mathrm{~s}$, max deflection $=0.0 \mathrm{in}$.

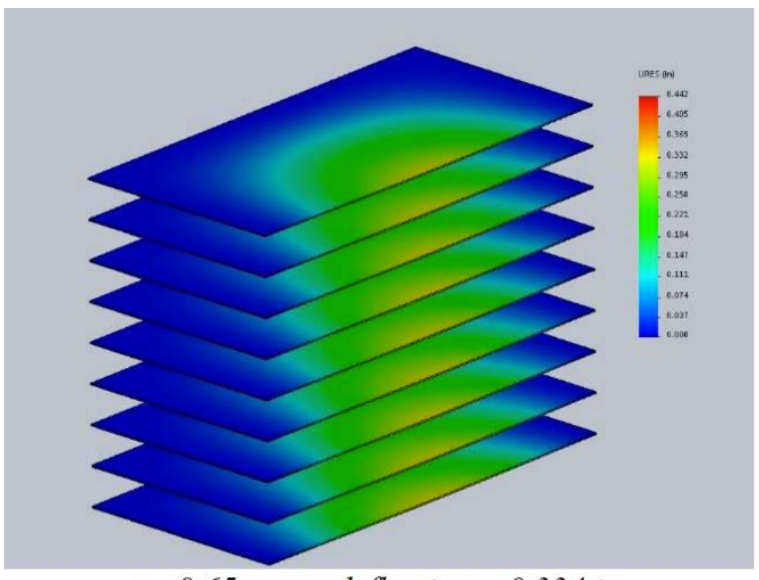

$t \approx 0.65$ s, max deflection $\approx 0.334 \mathrm{in}$.

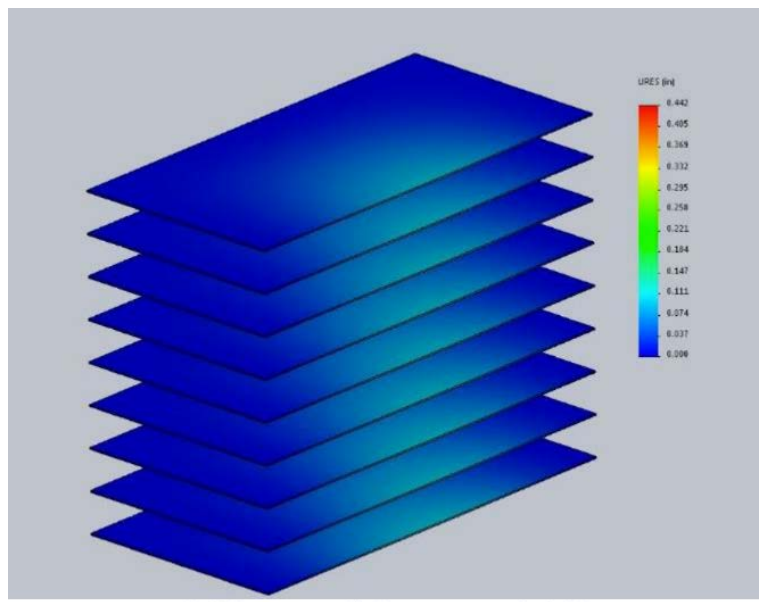

$t \approx 0.75 \mathrm{~s}$, max deflection $\approx 0.103 \mathrm{in}$.

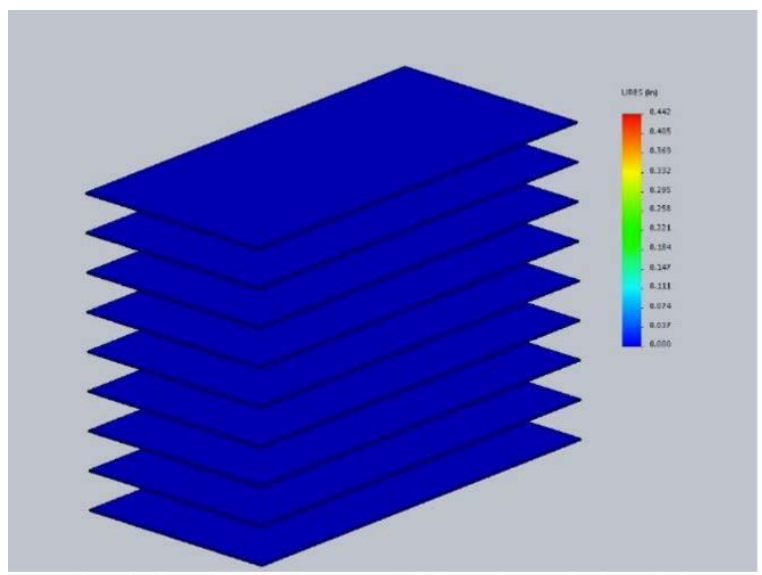

No deflection after $t=0.8 \mathrm{~s}$, plates unloaded.

Figure 120. Cessation of deflection of the $10^{\prime}$ x 5' x $1 / 2$ " plates under static and dynamic loading.

$(t=0.6$ to $t=0.9$ seconds $)$ 
Plate Design Iteration 6: Cross Beams Added to Plate for Additional Support

Model Details: $\quad$ A 10’ x 5’ x 3/8” plate was modeled with long edges fixed. Cross beams 1” x 1/4" x 10' were attached to the underside of the plate at 4' intervals (see Figure 121). A uniform pressure of 1.3 psi was applied over the face of the plate, accounting only for hydrostatic pressure.

FEA Results: Maximum von-Mises stress was observed to be 4,873 psi, (see Figure 122), with a maximum deflection of 0.101” (see Figure 123).

Remarks: $\quad$ Both maximum stress and deflection were considered acceptable; however, the interference of a horizontal support system with the target flow profile was unknown. Thin vertical supports would be a less invasive option.

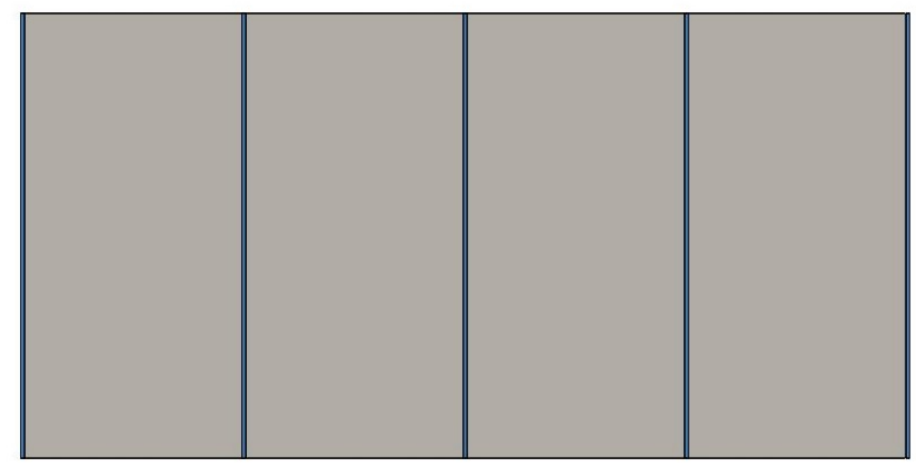

Figure 121. Bottom view of crossbeams attached to a 10’ x 5’ x 3/8” plate.

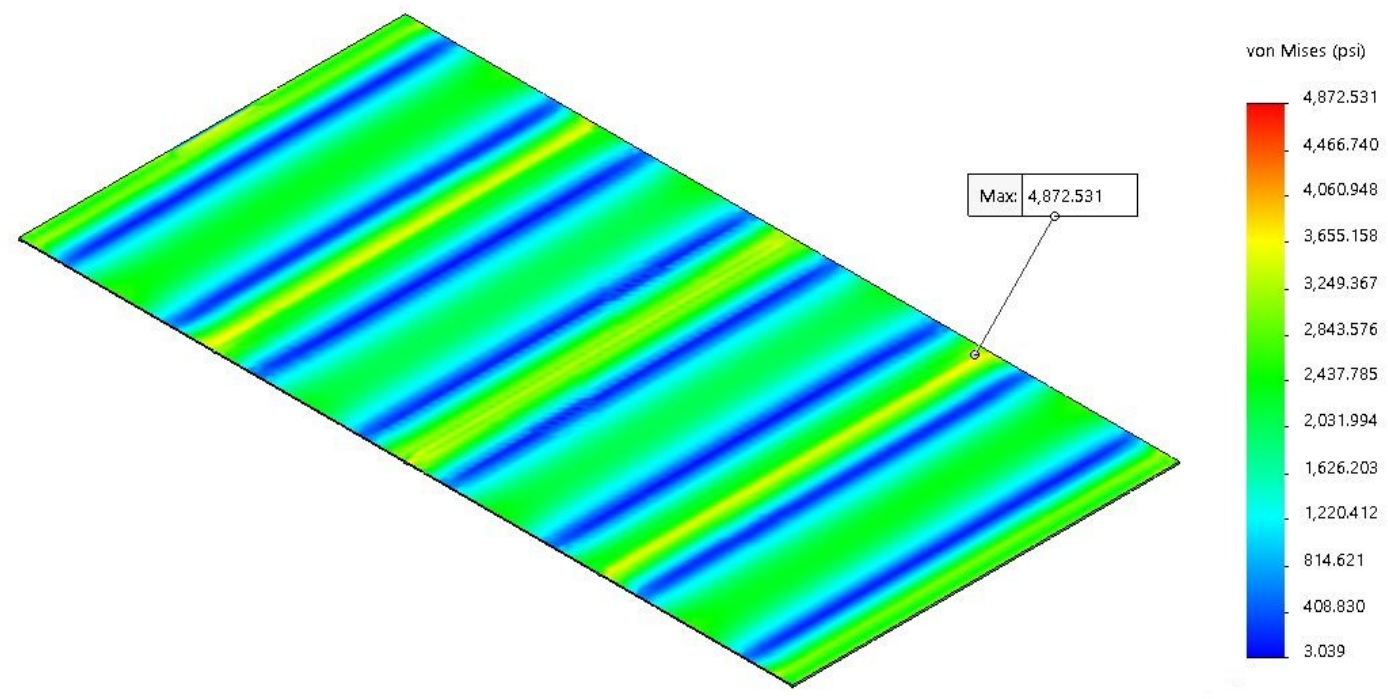

Figure 122. Von-Mises stress of a 10' x 5' x 3/8" plate with crossbeams under 1.3 psi. 


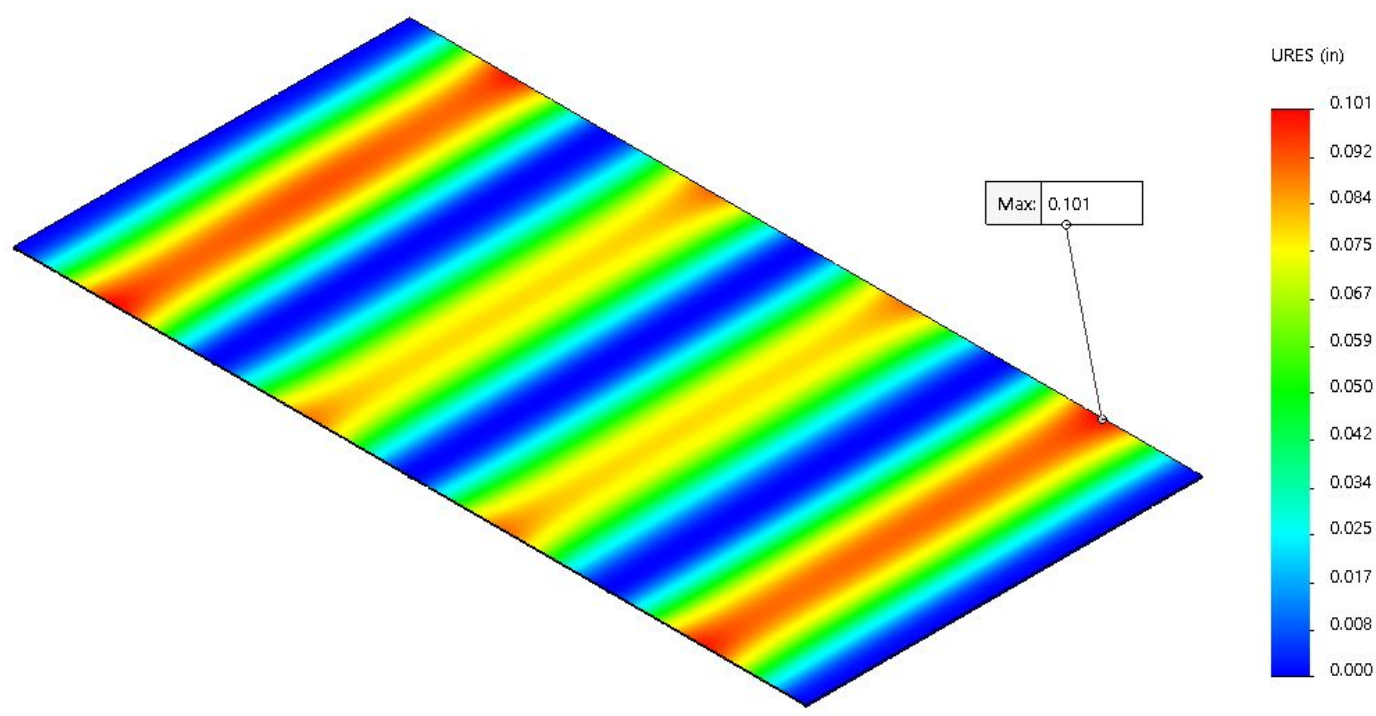

Figure 123. Deflection of a 10' x 5' x 3/8" plate with crossbeams under 1.3 psi.

Plate Design Iteration 7: A Thin Steel Support Installed at Exit Edge of Post-Gate Plates

Model Details: A 10' x 5' x 3/8” plate was modeled with long edges fixed in place. A 1/4” thick, steel vertical support was fixed to the plate using 3/8” notches at 12” intervals, (see Figure 124). A uniform pressure of 1.3 psi was applied over the face of the plate.

FEA Results: Maximum von-Mises stress was 17,074 psi and maximum deflection was 0.383”.

Remarks: $\quad$ The appearance of the stress concentration surrounding the vertical support was a concern for future cracking and/or failure in the support. But the overall improvement of placing a vertical support in the center of each plate was considered progress.

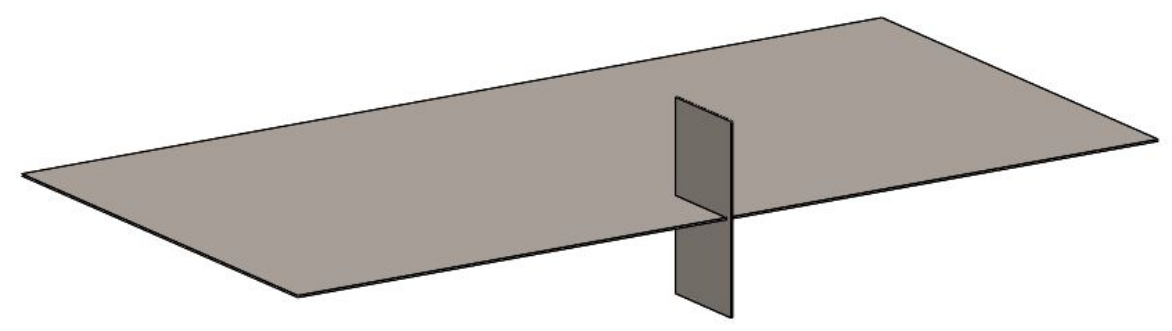

Figure 124. A 10’ x 5’ x 3/8” plate supported using 1/4” thick, notched steel. 
Plate Design Iteration 8: Thin Vertical Support Replaced by Two Rods

Model Details: $\quad$ A 10' x 5' x 3/8” plate was modeled with long edges fixed in place. The vertical support of Plate Design Iteration 8 was replaced by two 1/4" steel rods, fixed in tension to the top and bottom of the device housing (see Figure 125). Washers were attached to at each opening in the plate to provide reinforcement (see Figure 126). The rod was modeled as fixed to each washer and rod under the assumption that the components would be welded in the physical system. The distance from the edge of the plate to the first and second rod was 6” and 18”, respectively. A uniform pressure of 1.3 psi was applied over the face of the plate.

FEA Results: The maximum von-Mises stress was 16,254 psi (see Figure 127), and the maximum deflection was 0.049” (see Figure 128).

Remarks: $\quad$ High stress concentration were observed at the location of each fixture, even with the addition of the reinforcing washers. The deflection, however, was significantly decreased by 0.334 ”. It was hypothesized that additional rods would mitigate the stress concentrations but welding several threaded rods to each limited access plate was not feasible.

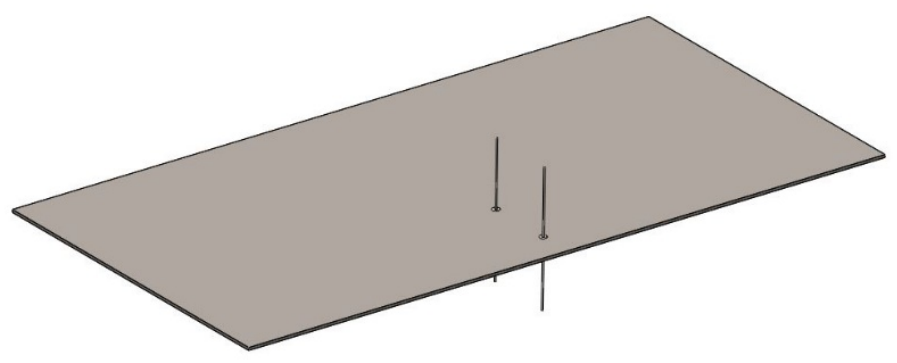

Figure 125. Overview of vertical rod plate support.

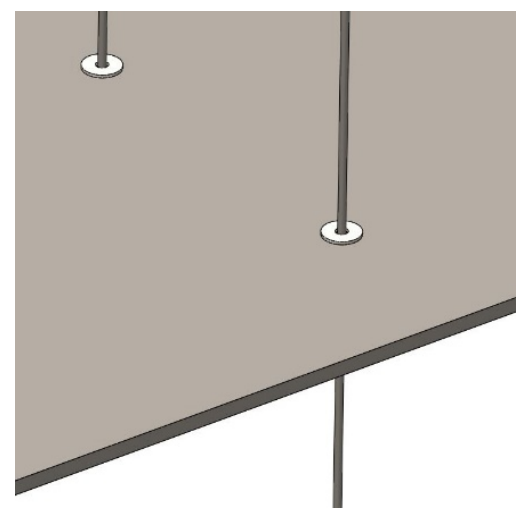

Figure 126. Close-up of vertical rod and washer attachments. 


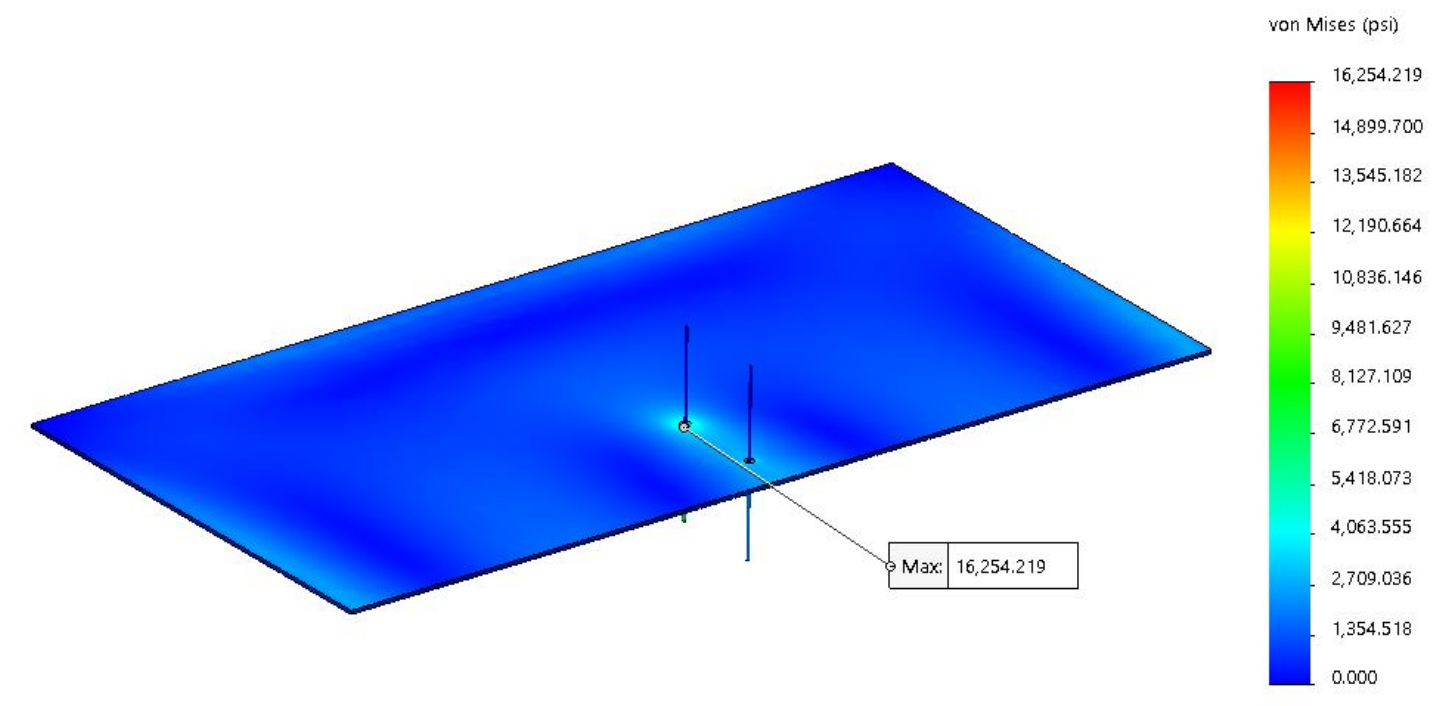

Figure 127. Von-Mises stress of a 10' x 5' x 3/8" plate under 1.3 psi with two support rods.

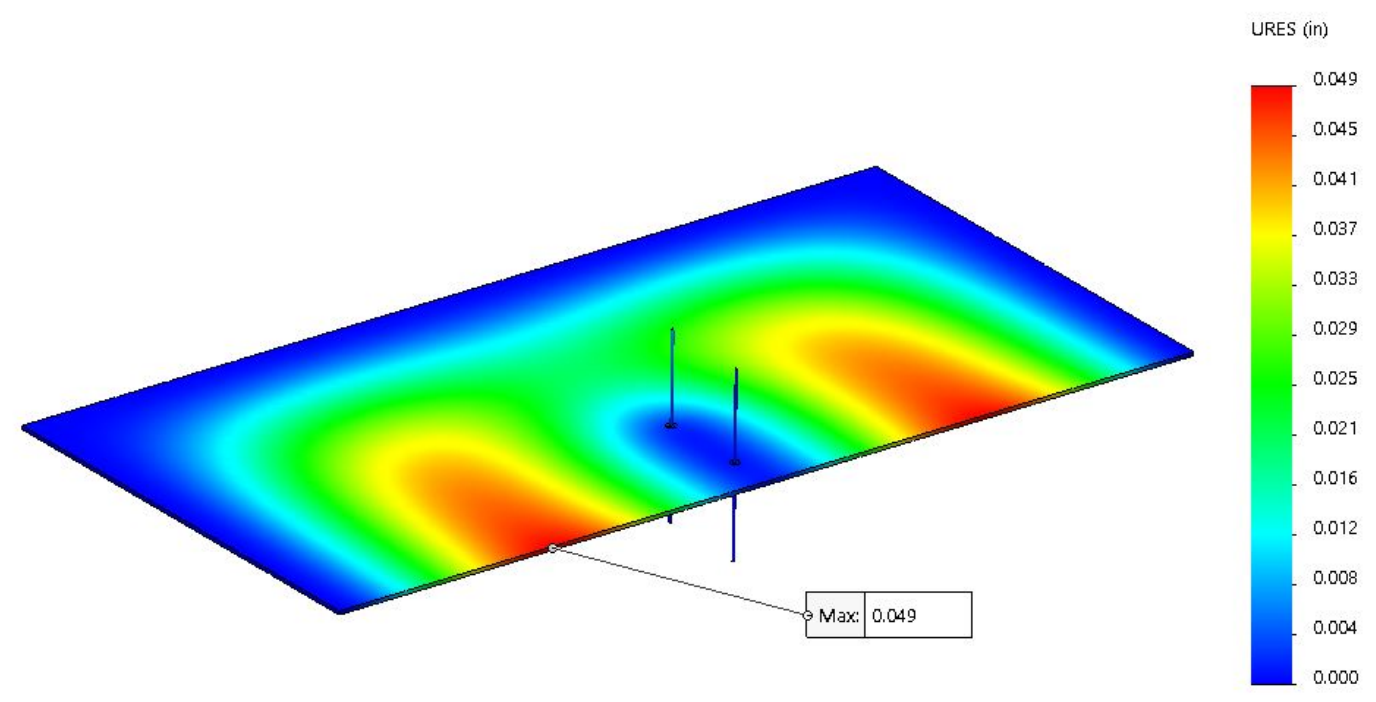

Figure 128. Deflection of a 10' x 5' x 3/8" plate under 1.3 psi with two support rods.

\section{Plate Design Iteration 9: Threaded Rods and Rivet Nuts for Additional Plate Support}

Model Details: $\quad$ One 10' x 5' x 3/8” was modeled with long edges fixed in place. Threaded rivet nuts were installed in intervals across each plate (see Figure 129). Six 1/4" steel threaded rods are threaded through each rivet nut, then fixed in tension to the top and bottom of the device housing (see Figure 130). The distance from the edge to the first and second rod in the center of the plate was 12" and 24", respectively. The distance from the edge to the first and second rod on each side of the center supports was 6” and 18”, respectively. A uniform pressure of 1.3 psi was applied over the face of the plate.

FEA Results: The maximum von-Mises stress was 15,801 psi (see Figure 131), and maximum deflection was 0.043 ” (see Figure 132). 
Remarks: $\quad$ The 15,801 psi maximum von-Mises stress in the plate was reduced to almost half the 36,000 psi yield stress of A36 steel. The 0.043” maximum deflection was small enough to be considered reasonably negligible. The concept of using thin, threaded rods coupled with lowprofile rivet nuts had succeeded in distributing the stress and deflection of each plate more uniformly than had previous design iteration. Thin vertical rods also meant less disruption to the wave development. The system was designed for each rod to be threaded continuously through each rivet nut in the plates below from above the WISD housing. This provided flexibility for future modifications since adding or removing supports could be done with ease as necessary.

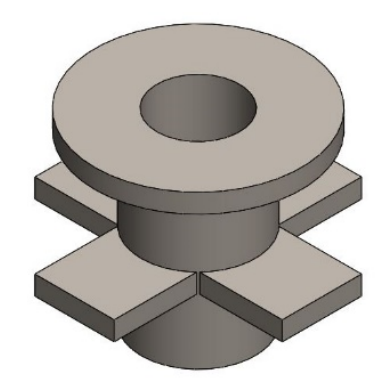

Figure 129. Close-up of expanded rivet nut after installation.

(Plate, rod, and threads removed for clarity.)

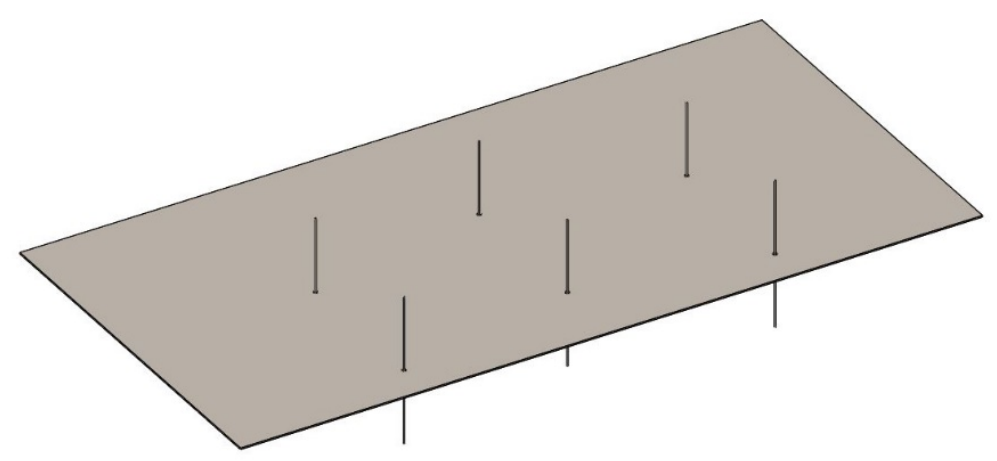

Figure 130. Overview of threaded rivet nut and rod plate supporting system. 


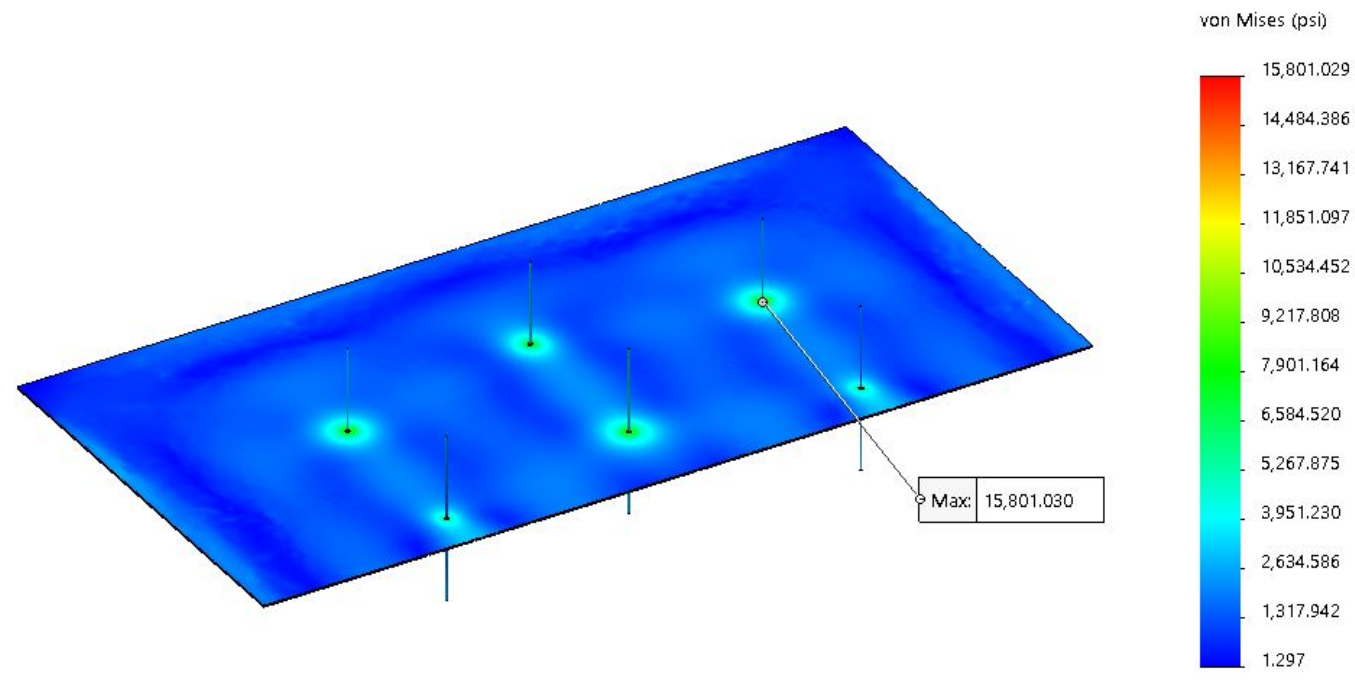

Figure 131. Von-Mises stress of a 10' x 5' x 3/8" plate under 1.3 psi with rivet nut/rod supports.

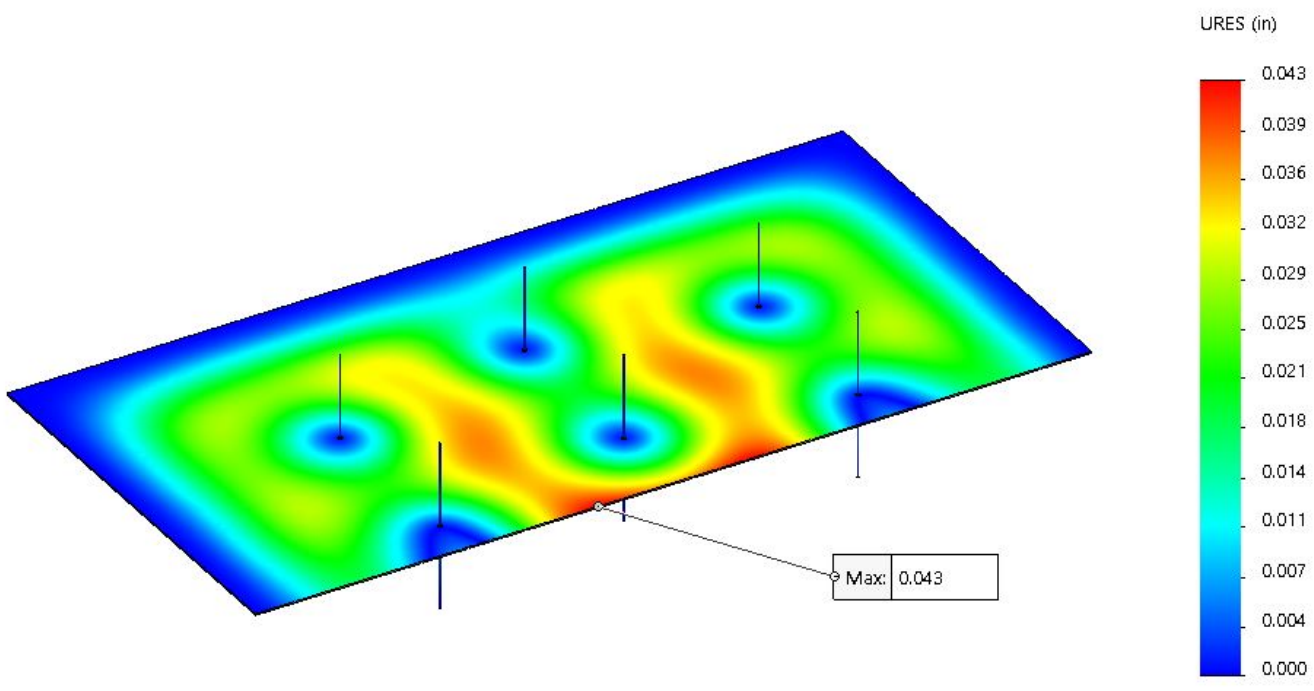

Figure 132. Deflection of a 10' x 5' x 3/8" plate under 1.3 psi with rivet nut/rod supports.

The success of the WISD is largely dependent on the functionality of the gate system. As part of the project requirements, the gate system must have the following capabilities:

1. Gates must be designed to withstand high water pressures before water is released.

2. Gate design should not interfere with the target flow profile.

3. All gates must open instantaneously.

4. Leakage from reservoir prior to gates opening should be kept at a bare minimum. 
The FLOW-3D simulations used the concept of ten bottom hinged-gates, opening each channel simultaneously as the water column behind them became pressurized. As such, each gate would be a rectangular valve driven by a single, rotating shaft. A gate thickness and shaft size capable of withstanding both hydrostatic and pneumatic pressures from the reservoir section would need to be determined. The amount of torque on the gate shaft was calculated under two main assumptions. First, the shaft was assumed fixed on each end (although the mechanism for holding the shaft in place had not yet been determined). And second, the gate would be permanently fixed to the shaft, and both components would be potentially subjected to 21.3 psi of hydrostatic and pneumatic pressure. Each gate must span the entire 10-ft. width of the channel; therefore, the resultant deflection and stress from the applied pressure would ultimately determine the configuration best suited for the task.

The method for attaching the gate to the shaft also presented the team with an obstacle. Since the shaft would need to drive the movement of the gate, the two required a strong, permanent bond. A keyed shaft is a standard rod designed with a machined groove running the length of the shaft to accommodate the placement of a key. The key is a small piece of metal fabricated to fit snugly into the groove of the shaft and accommodate other keyed components, such as gears and pulleys. The attached components can then be driven by the rotation of the shaft. Using the same concept, a thin plate of metal with a thickness equivalent to the respective key was assumed fixed into the keyway of the shaft using an all-around filet weld at the interface between shaft and plate. The shaft was designed to extend past the plate for 3" in each direction to allow space for the driving mechanism to be later installed. An overview of the simple gate created by the keyed shaft and plate is shown in Figure 133.
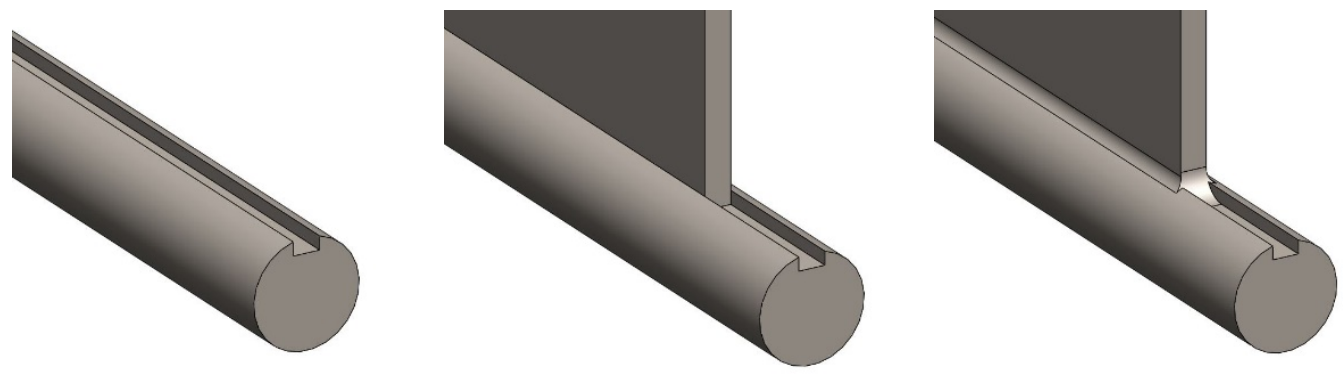

Figure 133. Isometric view of gate plate attached to keyed shaft.

(Left: keyed shaft, Center: plate inserted into keyway, Right: all around fillet weld)

The 10' channel span of the shaft, coupled with the 21.3 psi pressure acting on the gate from the water and compressed air would cause a large bending moment to occur on both pieces. To determine the extent of the bending moment, hand calculations from Shigleys Mechanical Engineering Design were used. The first calculation was performed to determine the minimum diameter required to withstand the 20 psi pneumatic pressure and 1.3 psi hydrostatic pressure using the full 36,000 psi yield strength of A36 steel. The resulting diameter was 3.88”. The next calculation removed the 20 psi pneumatic pressure to observe a minimum diameter requirement for only the 1.3 psi hydrostatic pressure, assuming the gates and compressed air could be released simultaneously. The reduction of pressure decreased the required rod diameter to 2.12". Further reduction of the hydrostatic pressure to 0.433 psi to account for only 1 ' head of head reduced the required shaft diameter to 0.71 ”.

The stress and deflection of the plate for all three pressure conditions was also determined. Using the same calculation approach as before, a 0.0416" rod was found to be sufficient for the 36,000 psi yield strength. However, the calculated deflection was 17.22”, a value not possible for a 12” gate. Unlike the shaft, the required gate thickness would be driven by the resultant deflection. This was address by decreasing the allowable deflection 
to 0.01 " (for minimal deflection), then calculating the required thickness to achieve it. The plate thickness required for the 0.01 ” deflection was approximately 0.499 ”.

After observing the stress and strain behavior of the shaft and gate as isolated components, it was then necessary to observe them as a single system since the hand calculations had assumed the attaching component was fixed. Five configurations using standard shaft sizes and corresponding plate thicknesses based on key width were modeled in SOLIDWORKS for FEA analysis. Standard keyed shaft dimensions from 1" to 2-1/2" are shown in Table 35.

Table 35. Standard Keyed Shaft Sizes (Grainger 2018)

\begin{tabular}{|c|c|c|c|}
\hline \multicolumn{2}{|c|}{ SHAFT DIAMETER (in.) } & \multicolumn{2}{c|}{ KEY WIDTH/PLATE THICKNESS (in.) } \\
\hline Fraction & Decimal & Fraction & Decimal \\
\hline 1 & 1 & $1 / 4$ & 0.25 \\
\hline $11 / 4$ & 1.25 & $1 / 4$ & 0.25 \\
\hline $11 / 2$ & 1.5 & $3 / 8$ & 0.375 \\
\hline $13 / 4$ & 1.75 & $3 / 8$ & 0.375 \\
\hline 2 & 2 & $1 / 2$ & 0.5 \\
\hline $21 / 4$ & 2.25 & $1 / 2$ & 0.5 \\
\hline $21 / 2$ & 2.5 & $5 / 8$ & 0.625 \\
\hline
\end{tabular}

Gate Design Iteration 1: 1” Keyed Shaft with a 1/4” Thick Rectangular Plate

Model Details: A 12” x 10' x 1/4" thick steel plate was fixed into the keyway of a 1" x 10-1/2' A36 steel keyed shaft. The shaft was fixed from rotation at each end, and a uniform pressure of 0.433 psi was applied perpendicular to the plate.

FEA Results: Deflection at the center of the gate was approximately 1.76” (see Figure 134).

Remarks: $\quad$ The $1.76 ”$ deflection was considered too large. 


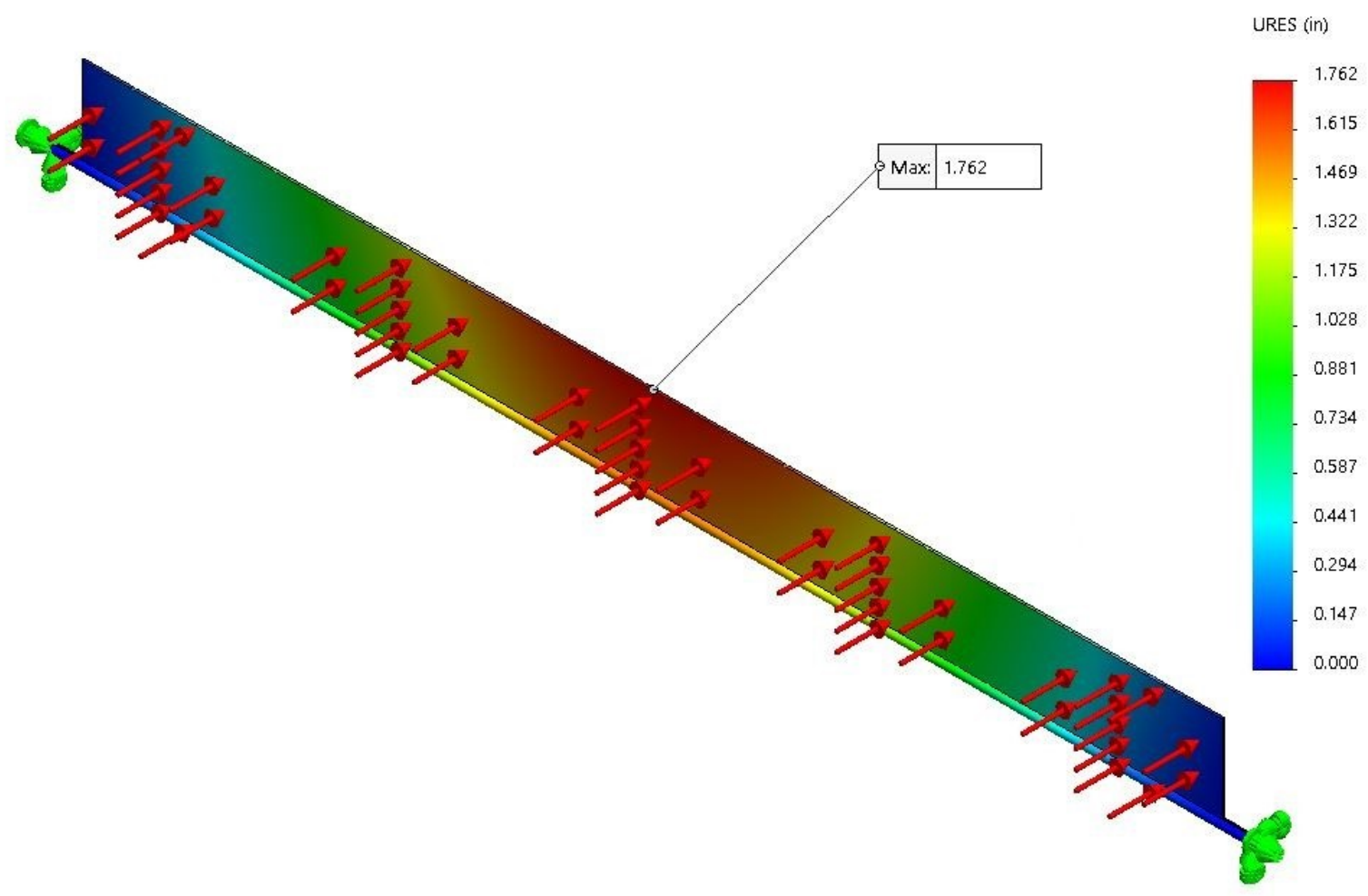

Figure 134. Deflection of a 1” keyed shaft with a 1/4" rectangular gate under 0.433 psi.

Gate Design Iteration 2: 1-1/4” Keyed Shaft with a 1/4” Thick Rectangular Plate

Model Details: A 12” x 10’ x 1/4" thick steel plate was fixed into the keyway of a 1-1/4" x 10-1/2' steel keyed A36 steel shaft. The shaft was fixed from rotation at each end, and a uniform pressure of 0.433 psi was applied perpendicular to the plate.

FEA Results: Deflection at the center of the gate was approximately 0.818” (see Figure 135).

Remarks: $\quad$ Deflection decreased from 1.762” to 0.818”, but less deflection was desired. 


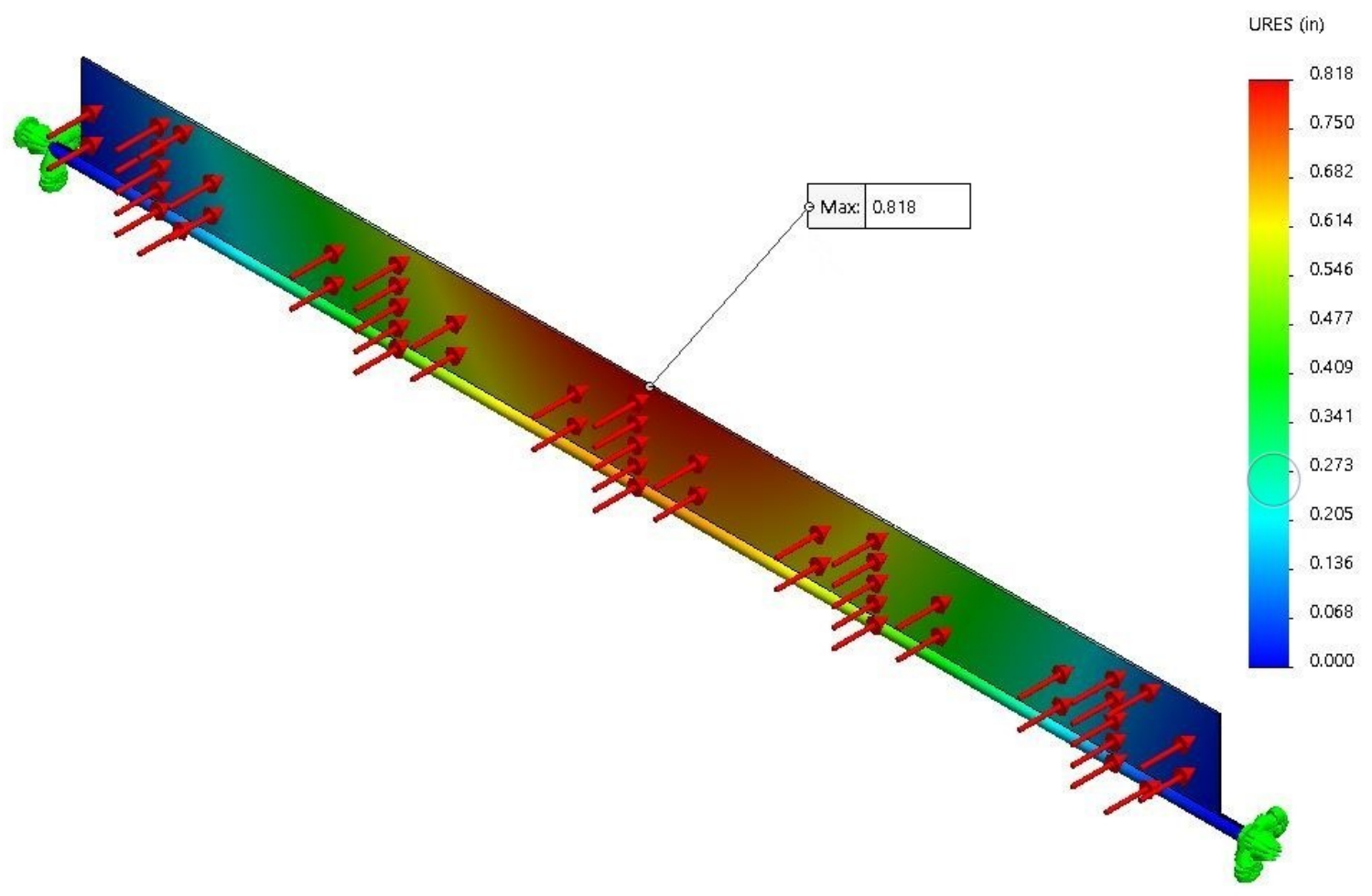

Figure 135. Deflection of a 1-1/4” keyed shaft with a 1/4" rectangular gate under 0.433 psi

\section{Gate Design Iteration 3: 1-1/2” Keyed Shaft with a 3/8” Thick Rectangular Plate}

Model Details: $\quad$ A 12” x 10’ x 3/8" thick steel plate was fixed into the keyway of a 1-1/2" x 10-1/2' A36 steel keyed shaft. The shaft was fixed from rotation at each end, and a uniform pressure of $0.433 \mathrm{psi}$ was applied perpendicular to the plate.

FEA Results: Deflection at the center of the gate was 0.375” (see Figure 139).

Remarks: $\quad$ Deflection was decreased from 0.818” to 0.375 ”, but team decided a deflection less than $1 / 4$ " would be optimal. 


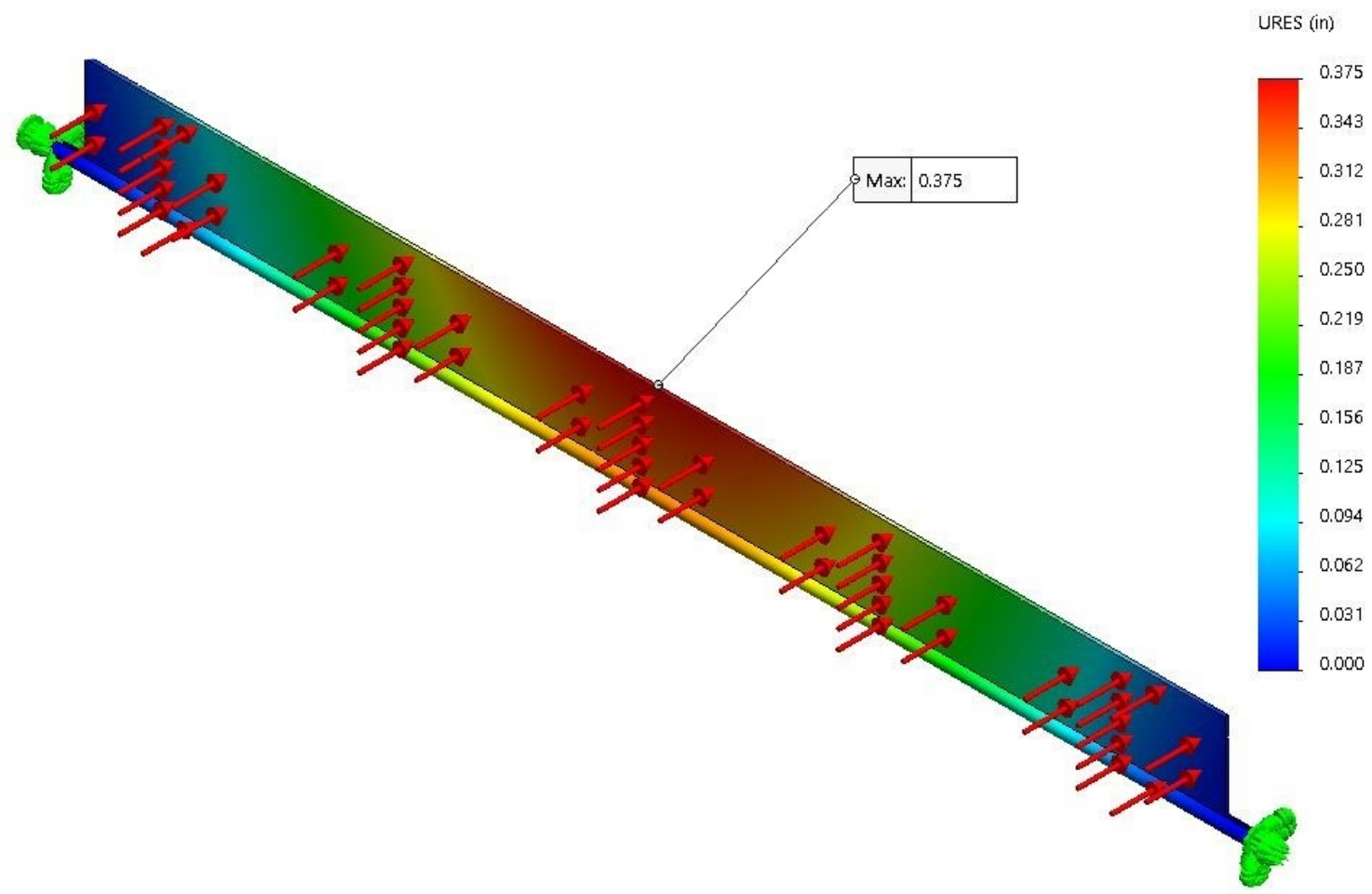

Figure 136. Deflection of a 1-1/2” keyed shaft with a 3/8" rectangular gate under 0.433 psi

Gate Design Iteration 4: 1-3/4” Keyed Shaft with a 3/8” Rectangular Plate

Model Details: $\quad$ A 12” x 10’ x 3/8" thick steel plate was fixed to the keyway of a 1-3/4" x 10-1/2' A36 steel keyed shaft. The shaft was fixed from rotation at each end, and a uniform pressure of $0.433 \mathrm{psi}$ was applied perpendicular to the plate.

FEA Results: Deflection at the center of the gate was 0.217” (see Figure 137).

Remarks: $\quad$ The results of the analysis showed the 1-3/4” shaft coupled with a 3/8” thick plate was an optimal configuration. The 0.217 " deflection of the gate was less than $1 / 4$ " and the $3 / 8$ " gate plate thickness would remain consistent with the surrounding WISD plates. Also, the 9,599 psi maximum von-Mises stress using the 1-3/4” keyed shaft and 3/8” thick rectangular plate yields a factor of safety of 3.8 (see Figure 138). 


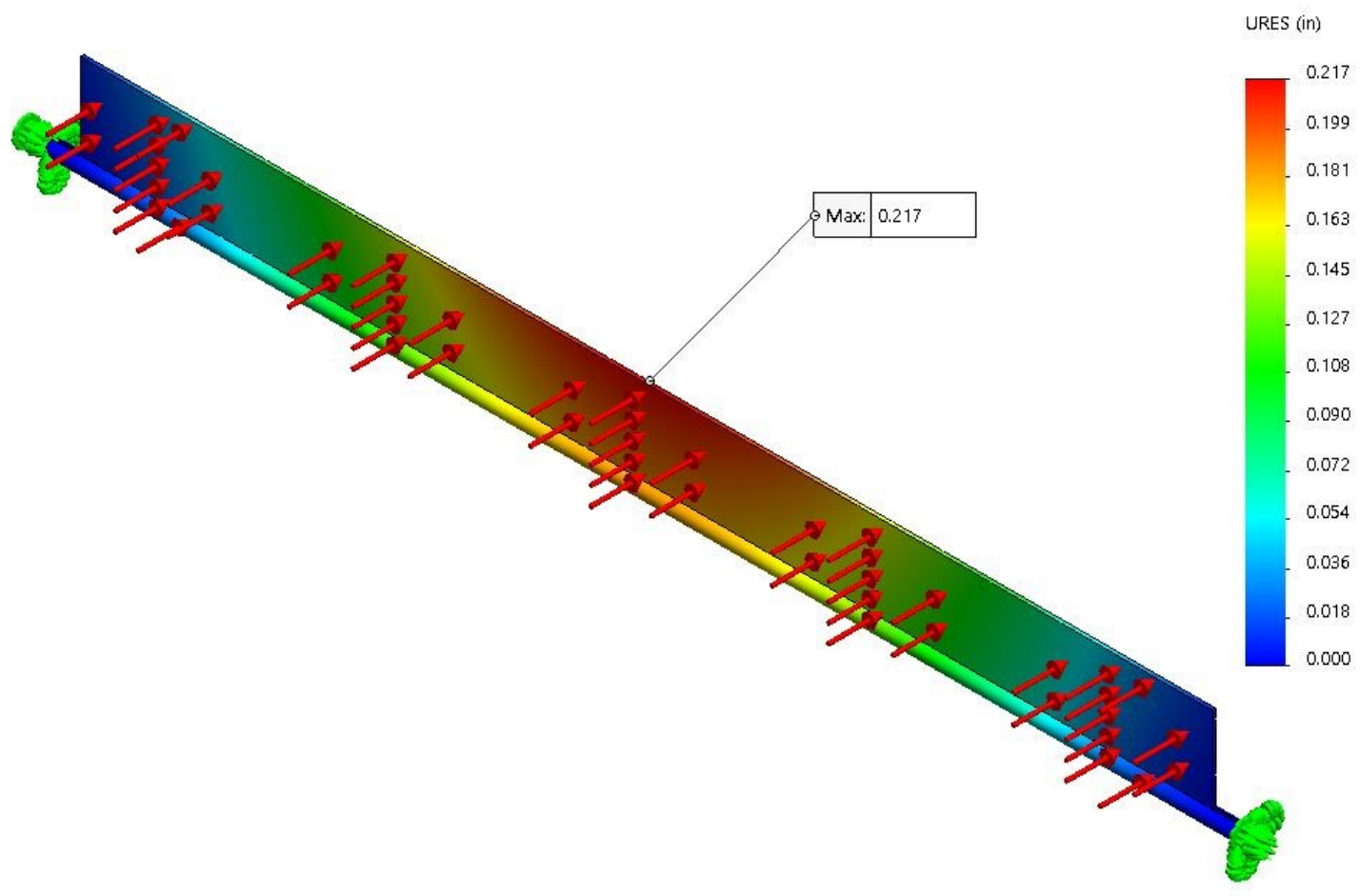

Figure 137. Deflection of a 1-3/4” keyed shaft with a 3/8" rectangular gate under 0.433 psi

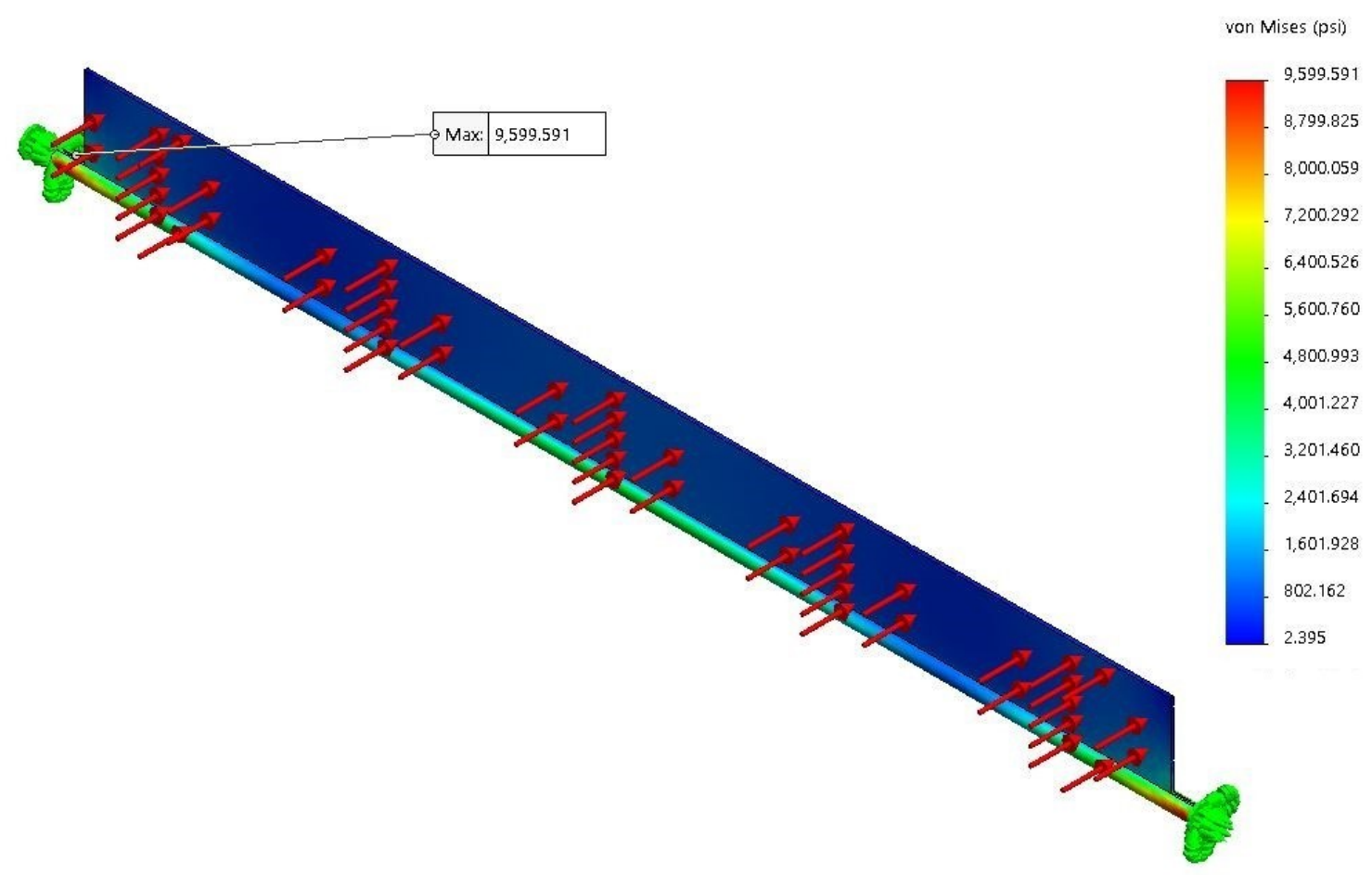

Figure 138. Von-Mises stress of a 1-3/4” keyed shaft with a 3/8" rectangular gate under 0.433 psi 
After establishing a 1-3/4" keyed shaft and a 3/8” thick gate plate would be adequate for the WISD prototype, one more design iteration was performed to determine if adding more support to each end of the shaft would further decrease the deflection by reducing the bending moment on the shaft. Custom shaft supports were designed to hinge each end of the shaft to the adjoining WISD plate. The addition of the two hinges did decrease the deflection approximately $10 \%$, from 0.217 ” to 0.196 ”, but the maximum von-Mises stress increased from 9,599.591 psi to 9,691.228 psi (about 1\%). The sole advantage of acquiring custom machined supports would be the minimal 0.021 " decrease in deflection. The team agreed pursuing the custom supports was not practical. However, an overview of the custom support design has been included below for future reference.

\section{Gate Design Iteration 5: 1-3/4” Keyed Shaft, 3/8” Thick Rectangular Plate, Custom Hinges}

Model Details: $\quad$ A 12” x 10’ x 3/8" thick steel plate was fixed into the keyway of a 1-3/4" x 10-1/2’ A36 steel keyed shaft. Two custom machined hinges were fixed at each end of the gate plate to the adjoining plate of the WISD, (see Figure 139, Figure 140, and Figure 141). The shaft was fixed from rotation at each end, and a uniform pressure of 0.433 psi was applied perpendicular to the plate.

FEA Results: Deflection at the center of the gate was reduced to 0.196” (see Figure 142). Maximum vonMises stress increased from to 9,691.228 psi, (see Figure 143)

Remarks: $\quad$ The cost associated with acquiring custom machined parts was not practical for the minimal 0.021 ” decrease in deflection. The concept was not pursued further.

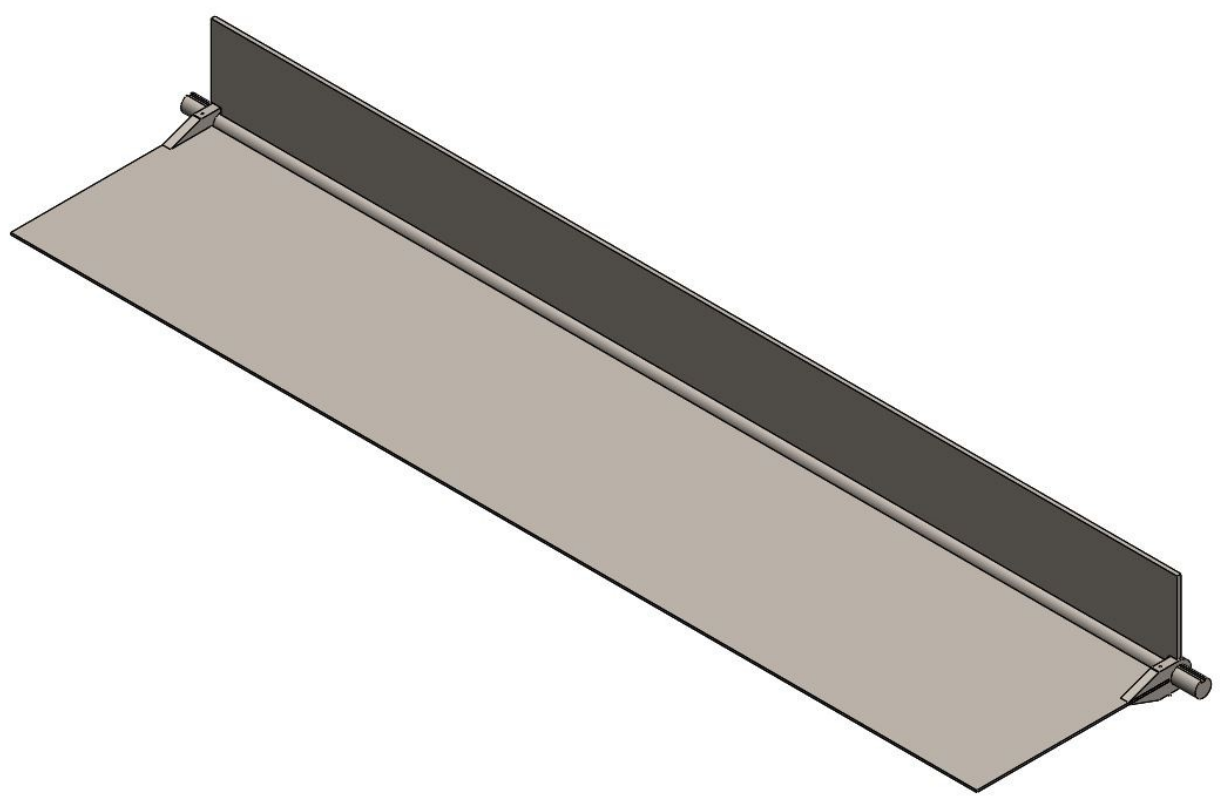

Figure 139. Isometric view of custom hinges supporting closed gate. 


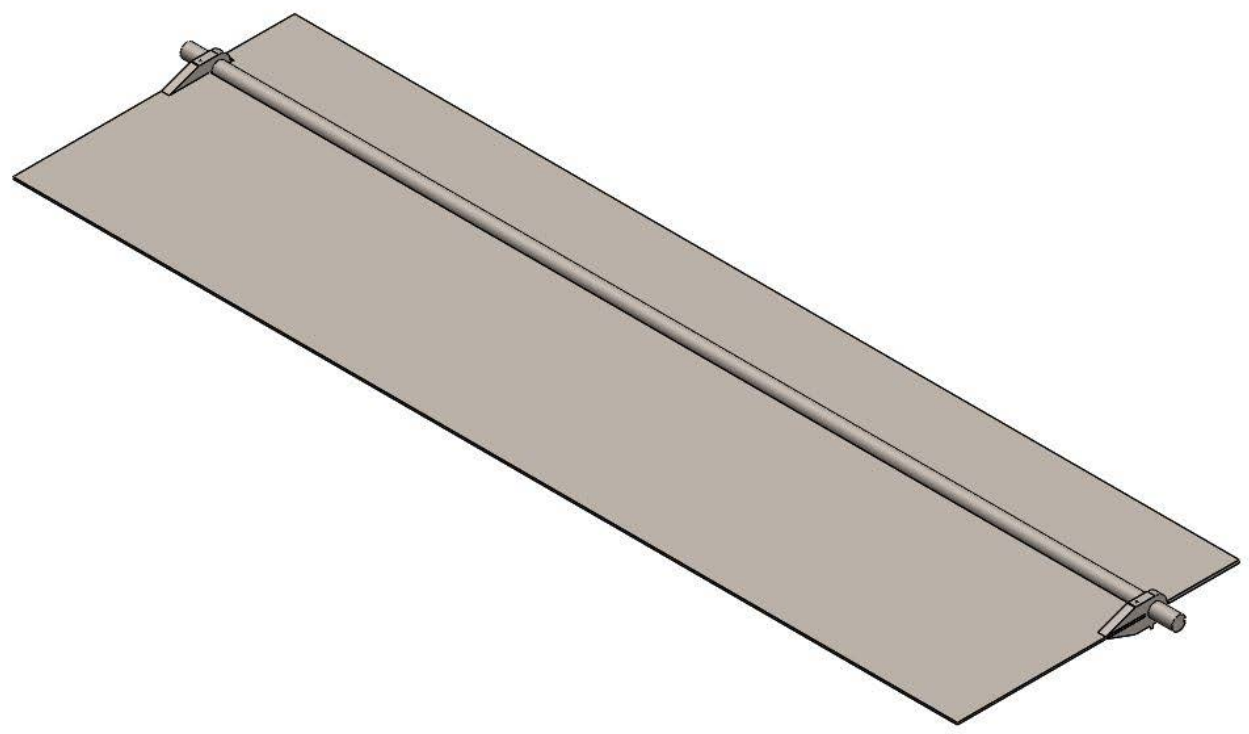

Figure 140. Isometric view of custom hinges supporting open gate
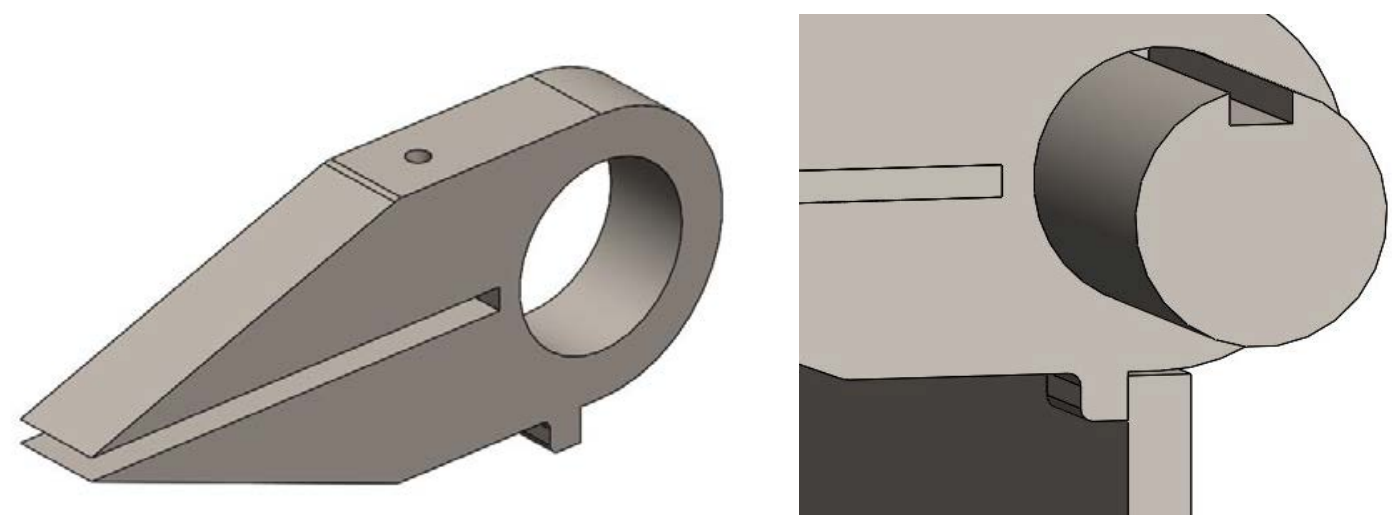

Figure 141. Close-up of custom machined hinge (left) and installation (right). 


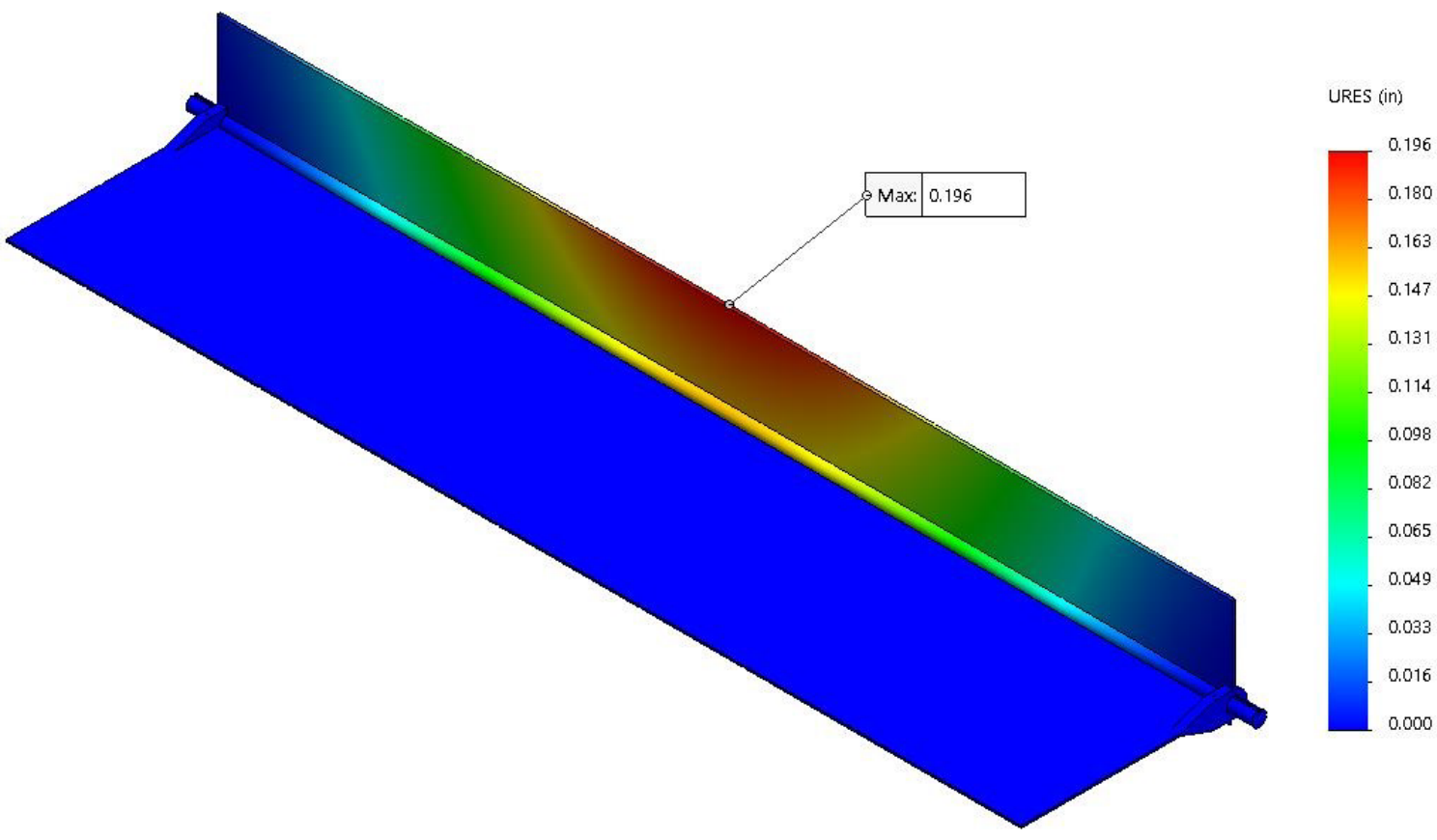

Figure 142. Deflection of 1-3/4” keyed shaft, 3/8" rectangular gate, and custom hinges under 0.433 psi.

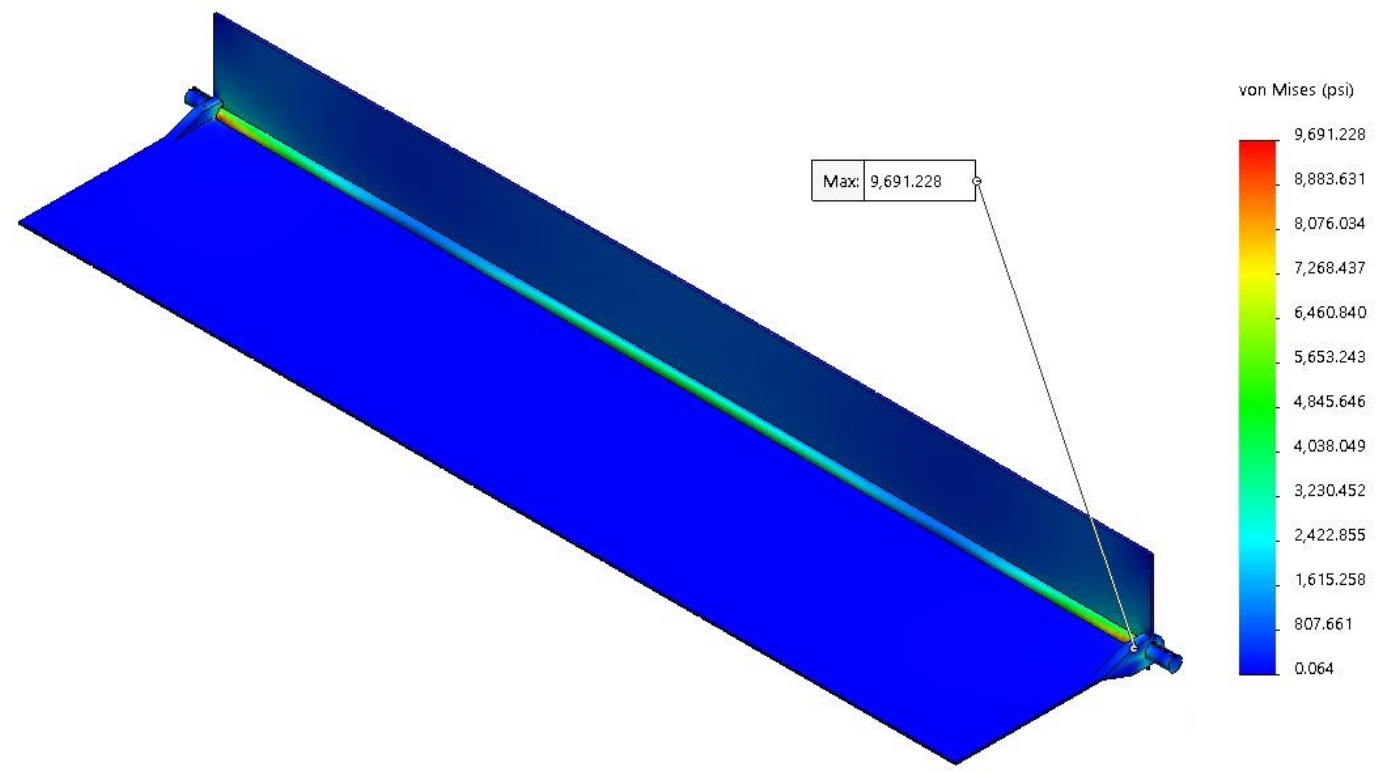

Figure 143. Von-Mises stress of 1-3/4” keyed shaft, 3/8" thick gate, and custom hinges under 0.433 psi.

\section{Overview of Structural Components Chosen for WISD Prototype}

Based on the results of the previous sections, the structural basis of the WISD prototype will consist of the following A36 plain carbon steel components:

$$
\begin{array}{ll}
\text { QTY }=10 & 1-3 / 4 ” \times 10-1 / 2 ’ \text { long keyed shaft (gate system) } \\
\text { QTY }=10 & 10 ’ \times 10-7 / 16 ” \times 3 / 8 ” \text { plate (gate system) } \\
\text { QTY }=9 & 10 ’ \times 5 ’ \times 3 / 8 ” \text { plate (plates after gate system) }
\end{array}
$$




$$
\begin{array}{ll}
\text { QTY }=9 & 10^{\prime} \times 10^{\prime} \times 3 / 8 ” \text { plate (plates before gate system) } \\
\text { QTY }=21 & 1 / 4^{\prime \prime} \times 126^{\prime} \text { threaded rod (plate support) } \\
\text { QTY }=21 & 1 / 4^{\prime \prime} \text { large flange, threaded rivet nut (plate support) }
\end{array}
$$

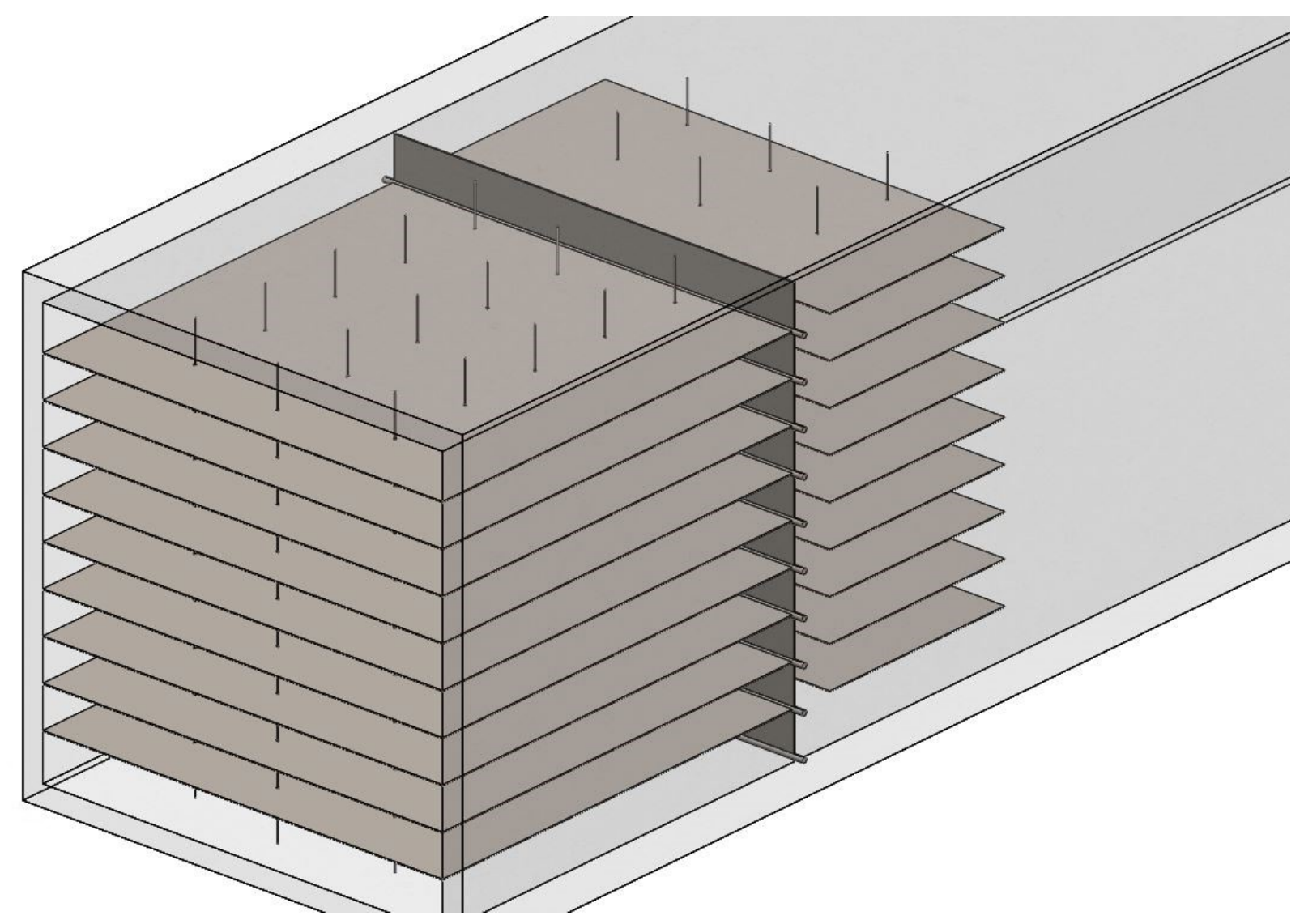

Figure 144. Simulation of chosen prototype components assembled within the WISD housing.

A key specification for the gate system was to ensure all ten gates to open as quickly as possible. In similar applications, fast response requirements are typically met using clutches or brakes. The issue with using these devices for the WISD gate mechanism is they operate principally on friction and can momentarily inhibit movement until the device is completely disengaged. The rotating gate shafts require a more immediate release from their static position to develop the angular velocity necessary to satisfy the rapid response requirement.

A more suitable option was to use electromagnets. Electromagnets, primarily electromagnetic locks, are commercially available with various holding force capacities. These devices are low profile, easy to operate, and quick to respond. While connected to a power source, an electromagnet is held fixed to its respective strike plate with a predesignated amount of holding force. The fail-open mechanism allows the magnet to immediately disengage from the strike plate the moment the power source is removed. Thus, a set of electromagnets affixed to each side of the gate shaft will hold all ten gates tightly closed while energized, then allow uninhibited, instantaneous rotation by simply interrupting power to the magnets.

To determine the total holding force required by each magnet, the 0.433 psi hydrostatic pressure of 1 ' head of water was multiplied by the 12" height of the channel and 120" width of the gate. The result was a 624 lbf required holding force per channel, or $312 \mathrm{lbf}$ holding force per magnet. However, to ensure a factor of safety of at least 2.0, two standard electromagnet locks with $650 \mathrm{lbf}$ holding force were specified for the final design. 
The angular velocity of the shaft after the magnet disengages from the strike plate was also calculated using the 1' head hydrostatic moment of $1248 \mathrm{lbf}$-in acting on the plate and a shaft diameter of 1-3/4". The angular velocity was found to be 90.76 radians/second with a total travel time of 0.0173 seconds for the entire 90 -degree rotation. Initially, it was thought a helical spring should also be incorporated into the gate mechanism to supplement the angular velocity; however, these calculations showed the moment created by 1' of hydrostatic head will provide more than sufficient torque to quickly rotate the shaft.

The electromagnets were integrated with the final WISD design using the 3" extension already designed into shaft of each gate. A 3/8” thick rectangular mounting plate was fixed to each 3" peripheral section assuming a welded connection, and the strike plates were attached to each mount using a single bolt. The electromagnet was secured to a secondary support system located on the outside of the WISD using machine screws. A 1" thick rubber damper was also installed on the secondary support system to help absorb the impact of the fast-moving gate as it reaches its final destination. Figure 145 and Figure 146 provide a simulated overview of the electromagnet operation. The WISD housing, plates, and supports have been removed for clarity.

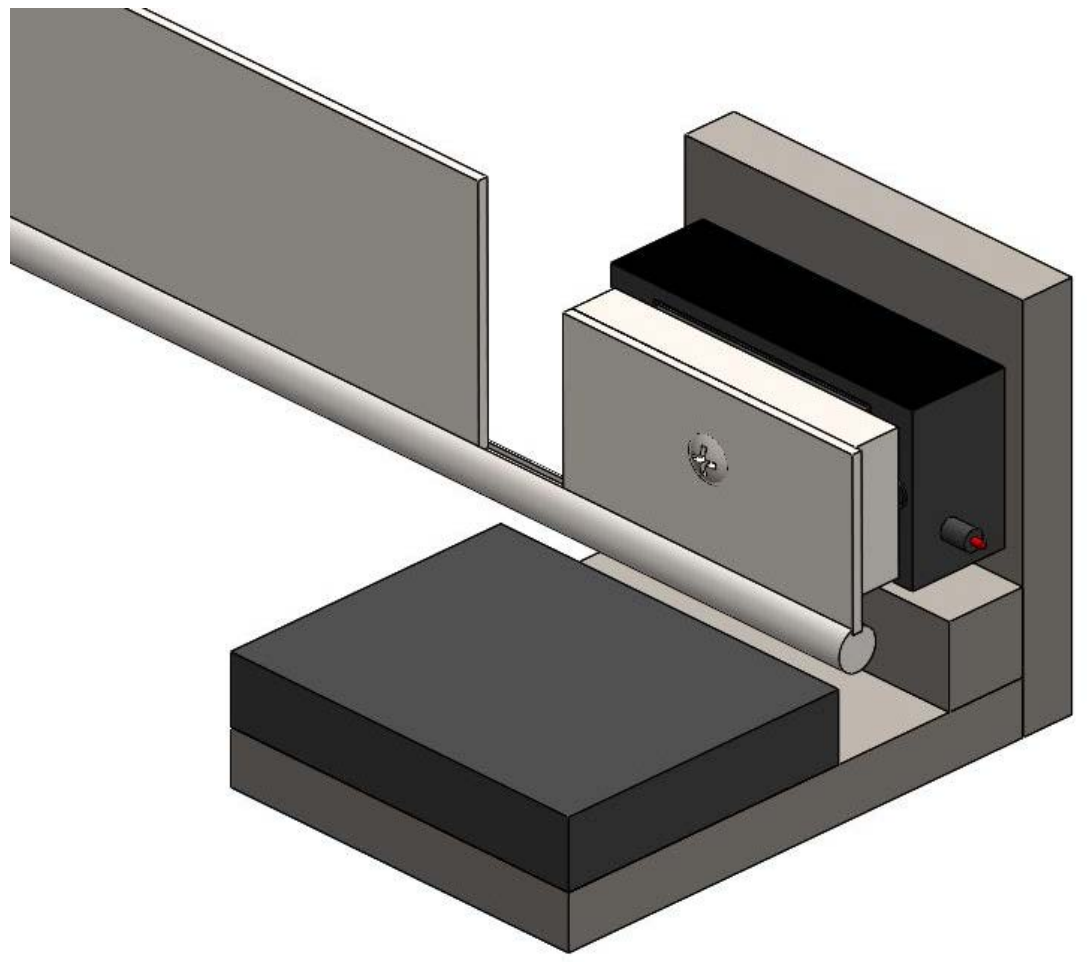

Figure 145. Simulation of the energized electromagnet holding gate in closed position. 


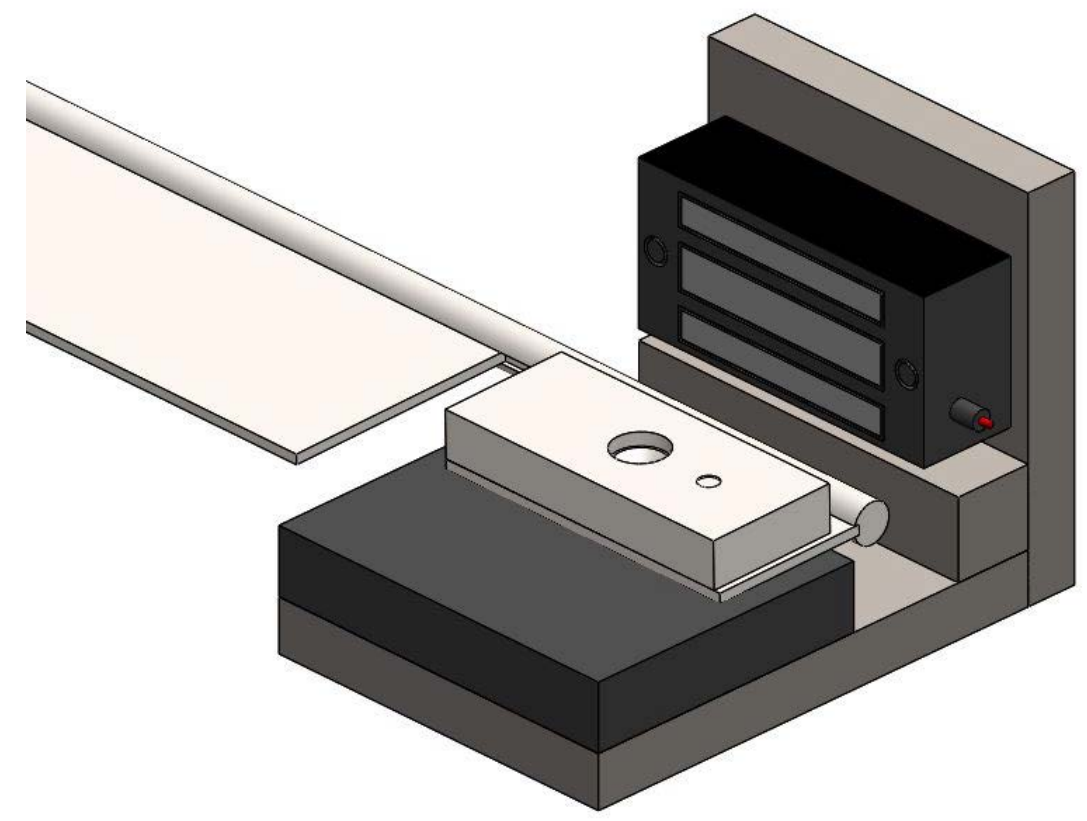

Figure 146. Simulation of the de-energized electromagnet releasing gate to opening position.

\section{CONCLUSIONS}

Tremendous progress has been made in all CFEL research areas. The progress includes performance improvements to the PET, PET based experiments using industrial steel doors, development of component fragility mathematical models, pipe rupture spray pattern characterization, coupling the SPH code Neutrino and RAVEN, wave impact simulation device development, analysis, and testing, and steering committee progress.

Testing involving inward opening steel doors will occur next in the PET followed by NPP fire barrier feedthroughs. Continued research on SPH particle spacing optimization using RAVEN is planned. Additionally, a benchmark quality pipe breach experiment is planned which will also be simulated with SPH to support particle spacing research as well as to provide additional code verification. Mathematical model development will continue and will use steel door related data collected from the PET experiments. WISD design and development will also continue and will use results from the research described in this report. Finally, a steering committee meeting will take place in the Summer of 2018. 


\section{REFERENCES}

[1] S. Bush, M. Do, A. Slavich and A. Chockie, "Piping Failures in United States Nuclear Power Plants: 19611995 (SKI-R--96-20)," Swedish Nuclear Power Inspectorate, Sweden, 1996.

[2] National Energy Board, "Vibration fatigue failure of piping (Safety Advisory NEB SA03-1)," National Energy Board, Calgary, 2003.

[3] National Fire Protection Association, NFPA 13: Standard for the Installation of Sprinkler Systems, Chicago: National Fire Protection Association, 2016.

[4] J. L. Coleman, C. Bolisetti, S. Veeraraghavan, C. Parisi, S. R. Prescott, A. Gupta and A. M. Kammerer, "Multi-Hazard Advanced Seismic Probabilistic Risk Assessment Tools and Applications (INL/EXT-1640055)," Idaho National Laboratory, Idaho Falls, 2016.

[5] "RAVEN," INL, [Online]. Available: https://raven.inl.gov/SitePages/Overview.aspx. [Accessed 8 Sept 2017]. 